\title{
The structure of galactic disks
}

\section{Studying late-type spiral galaxies using SDSS ${ }^{\star}$}

\author{
M. Pohlen ${ }^{1,2}$ and I. Trujillo 3,4 \\ 1 Kapteyn Astronomical Institute, University of Groningen, PO Box 800, 9700 AV Groningen, The Netherlands \\ e-mail: pohlen@astro.rug.nl \\ 2 Instituto de Astrofísica de Canarias, C/ Via Láctea s/n, 38200 La Laguna, Tenerife, Spain \\ 3 Max-Planck-Institut für Astronomie, Königstuhl 17, 69117 Heidelberg, Germany \\ 4 School of Physics and Astronomy, University of Nottingham, University Park, Nottingham NG7 2RD, UK \\ e-mail: ignacio.trujillo@nottingham.ac.uk
}

Received 20 January 2006 / Accepted 26 April 2006

\section{ABSTRACT}

\begin{abstract}
Using imaging data from the SDSS survey, we present the $g^{\prime}$ and $r^{\prime}$ radial stellar light distribution of a complete sample of $\sim 90$ face-on to intermediate inclined, nearby, late-type $(\mathrm{Sb}-\mathrm{Sdm})$ spiral galaxies. The surface brightness profiles are reliable ( $\sigma$ uncertainty less than $0.2 \mathrm{mag}$ ) down to $\mu \sim 27 \mathrm{mag} / \square^{\prime \prime}$. Only $\sim 10 \%$ of all galaxies have a normal/standard purely exponential disk down to our noise limit. The surface brightness distribution of the rest of the galaxies is better described as a broken exponential. About $60 \%$ of the galaxies have a break in the exponential profile between $\sim 1.5-4.5$ times the scalelength followed by a downbending, steeper outer region. Another $\sim 30 \%$ shows also a clear break between $\sim 4.0-6.0$ times the scalelength but followed by an upbending, shallower outer region. A few galaxies have even a more complex surface brightness distribution. The shape of the profiles correlates with Hubble type. Downbending breaks are more frequent in later Hubble types while the fraction of upbending breaks rises towards earlier types. No clear relation is found between the environment, as characterised by the number of neighbours, and the shape of the profiles of the galaxies.
\end{abstract}

Key words. galaxies: photometry - galaxies: structure - galaxies: fundamental parameters - galaxies: evolution - galaxies: formation

\section{Introduction}

The structural properties of the faintest regions of galactic disks must be intimately linked to the mechanisms involved in the growing and shaping of galaxies. These outer edges are easily affected by interactions with other galaxies and, consequently, their characteristics must be closely connected with the evolutionary path followed by the galaxies. Together with their stellar halos, the study of the outer edges allows the exploration of the so-called fossil evidence imprinted by the galaxy formation process.

Most of the so far detailed structural studies of nearby disk galaxies (Courteau 1996; de Jong 1996; Graham 2001; Jansen \& Kannappan 2001; Trujillo et al. 2002; MacArthur et al. 2003; Möllenhoff 2004) have been concentrated on the (most easily accessible) inner parts of the galaxies. These studies of the brightest region of the stellar disk show that its light distribution is almost always well described by a simple exponential decline going back to Patterson (1940), de Vaucouleurs (1959), or Freeman (1970). This simple description, however, has now been shown to fail at fainter surface brightness. In fact, since van der Kruit (1979) we know that this decline does not continue to the last measured point, but is truncated after several radial scalelengths.

The main concern with current, larger statistical studies exploring the faintest surface brightness region of the galaxies is

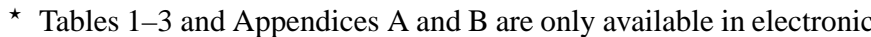
form at http: //WwW. edpsciences.org that they are based on edge-on galaxies (Barteldrees \& Dettmar 1994; Pohlen et al. 2000a; Florido et al. 2001; de Grijs et al. 2001; Pohlen 2001; Kregel et al. 2002). This geometry facilitates the discovery of the truncation in the surface brightness profiles, but introduces severe problems caused by the effects of dust and the line-of-sight integration (Pohlen et al. 2004, for a recent review), such as masking the actual shape of the truncation region, or interfering with the identification of other important disk features (e.g. bars, rings, or spirals).

Only few works have probed the faintest regions of the disk galaxies in low inclination systems. Pohlen et al. (2002) demonstrated for a small sample of face-on galaxies that the socalled cut-offs in the surface brightness profiles discovered by van der Kruit are in fact not complete but better described by a broken exponential with a shallow inner and a steeper outer exponential region separated at a relatively well defined break radius. However, not all the galaxies seem to have a break or a truncation in their surface brightness profiles. Just recently Bland-Hawthorn et al. (2005) found (using star counts) again a galaxy (NGC 300) for which the exponential decline simply continues down to $\sim 10$ radial scalelength. Together with earlier measurements by Barton \& Thompson (1997) or Weiner et al. (2001) (using surface photometry) this provides evidence that indeed there are prototypical, model exponential disks.

Our picture of the faintest regions of spiral galaxies have been even broadened with some recent studies. Erwin et al. (2005a), studying a large sample of early-type barred S0$\mathrm{Sb}$ galaxies, discovered that there is more than truncated or 
untruncated galaxies. They report the detection of antitruncated galaxies, which show also a broken exponential but having the opposite behaviour, an outer upbending profile. Similar structures are also found for a large sample of irregular systems (Im) and BCDs by Hunter \& Elmegreen (2006).

The disk structures we describe in this study are the result of initial conditions, infalling matter and/or redistribution of gas and stars, already settled in the disk, triggered by internal (e.g. bars) or external (e.g. interaction) forces. All these processes are linked to the detailed mechanism of forming the stars which build up the observed brightness distribution. To explain for example the observed truncation in the surface brightness profiles radial star-formation thresholds (e.g. Kennicutt 1989; Schaye 2004) have been suggested (see Pohlen et al. 2004). However, there is compelling evidence of star-formation in the far outer regions of spiral galaxies (e.g. Ferguson et al. 1998; Cuillandre et al. 2001; Thilker et al. 2005; Gil de Paz et al. 2005), well beyond the break of the observed broken exponential structure (Pohlen et al. 2002). The outer star formation activity argues against a simple threshold scenario. Just recently, though, Elmegreen \& Hunter (2006) show that their model of star-formation is able to produce the variety of observed radial profiles. Alternatively, Debattista et al. (2006) also find downbending breaks using purely collisionless $N$-body simulations.

The main goal of the present study is to conduct a complete census of the outer disk structure of late-type galaxies - together with a complementary study by Erwin et al. (2006) for earlytype (barred) galaxies - in the local universe. The objective is to provide the frequencies of the different surface brightness profile types, the structural disk parameters, and search for correlations (if any) between them.

Aside from the observations showing the increasing complexity of radial surface brightness profiles, the breaks in the surface brightness distribution can be used to directly constrain galaxy evolution. Pérez (2004) showed that it is possible to detect stellar truncations even out to higher redshift $(z \sim 1)$. So using the radial position of the truncation as a direct estimator of the size of the stellar disk, Trujillo \& Pohlen (2005) infer a moderate $(\sim 25 \%)$ inside-out growth of the disk galaxies since $z \sim 1$, using as a local reference the galaxies studied in this paper.

The remainder of this paper is organised as follows. In Sect. 2 we describe the sample selection and our environment parameter. In Sect. 3 we give the details about the ellipse fitting and the SDSS imaging used, characterising their quality and addressing the crucial issue of sky subtraction. The applied classification schema of the observed profiles and the derived parameters are discussed in Sect. 4. The results are presented in Sect. 5 and briefly discussed in Sect. 6 . The colour profiles and the physical implications derived from them will be discussed in a subsequent paper. In Appendices A we give detailed comments for all galaxies, show their $r^{\prime}$ and $g^{\prime}$-band surface brightness profiles and reproduce their SDSS colour pictures.

\section{The sample}

\subsection{Selection}

We selected our initial galaxy sample from the LEDA ${ }^{1}$ online galaxy catalogue (version Nov. 2004), since this is the richest catalogue with homogeneous parameters of galaxies for the largest available sample. We restricted the Hubble type ( $T$ parameter), the mean heliocentric radial velocity relative to the

\footnotetext{
${ }^{1}$ http://leda.univ-lyon $1 . f r$
}

Local Group (corrected for virgocentric inflow, $v_{\text {vir }}$ ), the axis ratio (major axis/minor axis), the absolute $B$-magnitude $\left(M_{\mathrm{abs}}\right)$, and the Galactic latitude $\left(b_{\mathrm{II}}\right)$.

The Hubble type is chosen to be between $2.99<T<8.49$ (corresponding to $\mathrm{Sb}$ to $\mathrm{Sdm}$ galaxies) building an intermediateto late-type galaxy sample. This allows a complementary study to the work by Erwin et al. (2006) on the disk structure of early type (barred) galaxies. To have some, but not too much, overlap with their sample we did not include all $\mathrm{Sb}$ galaxies by excluding the range $2.49<T<2.99$. The axis ratio is selected to be $\log r_{25}<0.301$ (equal to $a / b<2$ or $e<0.5$, which corresponds to an inclination of $\lesssim 61^{\circ}$ assuming an intrinsic flattening of $q_{0}=0.14$ ) bringing a face-on to intermediate inclined galaxy sample. This is necessary to avoid the influence of the dust and is convenient to provide reliable information on the morphological features such as bars, rings, or spiral arm structure. The recession velocity is chosen to be $v_{\text {vir }}<3250 \mathrm{~km} \mathrm{~s}^{-1}$ and the galaxies brighter than $-18.4 M_{\text {abs }}$ in $B$ band (the total $B$-magnitude, provided by LEDA, is corrected for galactic and internal extinction, $k$ corrected, and the distance modulus is derived from the recession velocity corrected for Virgo infall with a Hubble constant of $H_{0}=70 \mathrm{~km} \mathrm{~s}^{-1} \mathrm{Mpc}^{-1}$ ). We did not apply a diameter limit. Since SDSS, our data source, is a survey of the high Galactic latitude sky, visible from the northern hemisphere, there is no worry about incompleteness there. In any case, we restricted the initial sample to $\left|b_{\mathrm{II}}\right|>20^{\circ}$.

To assess the completeness of the LEDA catalogue Baryshev $\&$ Paturel (2001) draw several cumulative curves $\log N$ versus $\log r$ ( $N$ is the total number of observed galaxies within the radius $r$ in $\mathrm{Mpc}$ ) for different absolute magnitudes and compare these with an expected galaxy distribution. Using the same approach we have confirmed that LEDA (version Nov. 2004) is almost complete for galaxies of $M_{\mathrm{abs}}<-18.4$ within our $\sim 46 \mathrm{Mpc}$ survey distance (estimated following the Hubble relation with $\left.H_{0}=70 \mathrm{~km} \mathrm{~s}^{-1} \mathrm{Mpc}^{-1}\right)$.

Our selection criteria result in a total number of 655 galaxies, of which $98(15 \%)$ are part of the SDSS Second Data Release (DR2), surveying the north Galactic cap and three stripes in the southern Galactic cap (York et al. 2000; Abazajian et al. 2004). The global parameters ${ }^{2}$ for this subsample of 98 galaxies are presented in Table 1. Histograms showing the distribution of Hubble type and absolute magnitude (cf. Fig. 1) reveal that the subsample restricted by the SDSS DR2 coverage is apparently unbiased in this sense. There is a lack of very bright galaxies $\left(M_{\mathrm{abs}}<-21.9 B\right.$-mag) due to the small number statistics. Plotting absolute magnitude $M_{\text {abs }}$ with Hubble Type $T$ reveals although with a lot of scatter - a mild trend of earlier Hubble type galaxies being on average brighter.

Only three galaxies (NGC 4273, NGC 4480, NGC 4496A) are possible members of the Virgo Cluster according to their VCC numbers by Binggeli et al. (1985). In this sense our sample is a faithful representation of the local spiral galaxy population providing a volume limited sample ( $D \lesssim 46 \mathrm{Mpc}$ ) of late-type, intermediate inclined to face-on nearby galaxies brighter than $-18.4 B$-mag. The Appendices A and B show colour images for all galaxies.

\footnotetext{
${ }^{2}$ Note: some of the bar classifications from LEDA have slightly changed after our study. Table 1 still shows the 2004 version. For comparison we always listed the RC3 classification.
} 

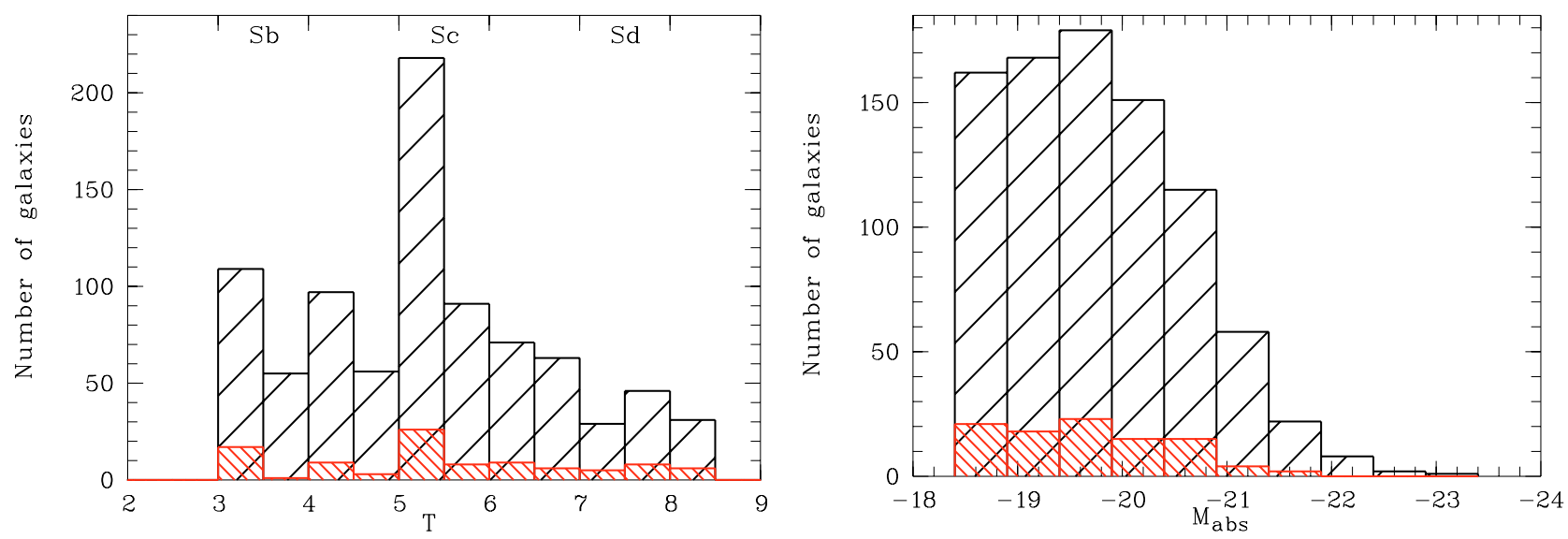

Fig. 1. Histogram showing the distribution of galaxies covering the morphological types $(T)$ and absolute magnitude $\left(M_{\mathrm{abs}}\right)$ for the full LEDA sample (rising hashed lines) and the studied SDSS DR2 subsample (falling hashed lines).

\subsection{Environment}

To characterise the environment we counted neighbours around our sample galaxies using the SDSS database from a more recent Data Release (DR3, Abazajian et al. 2005). According to the distance, derived from the infall corrected velocity, we sum all the DR3 galaxies within a projected radius of $1 \mathrm{Mpc}$ that satisfy the following criteria: the difference in velocity with respect to the targeted galaxy is less than $350 \mathrm{~km} \mathrm{~s}^{-1}$ (Giuricin et al. 2000) and their absolute magnitude is brighter than $M_{\text {abs }}<-16 r^{\prime}$-mag. It is important to note, however, that the density of galaxies could be affected by the position of the galaxy within the survey. If the targeted galaxy is close to the edge of the survey or to a region where the redshift completeness (fraction of photometrically detected galaxies with redshifts) is not high, the number of neighbours could be underestimated. To eliminate such a problem we have estimated whether the distance of our galaxy to the edge of the survey or to a redshift incomplete region is less than $1 \mathrm{Mpc}$. If this is the case this galaxy is not used in the analysis of the effect of the density on the galaxy profile type.

\section{Data and profile extraction}

\subsection{The data}

The Sloan Digital Sky Survey (SDSS) (York et al. 2000) will map one-quarter $\left(\sim 10^{4} \square^{\circ}\right)$ of the entire sky mainly around the north galactic cap (above Galactic latitude $\sim 30^{\circ}$ ) in five bands, $u^{\prime}, g^{\prime}, r^{\prime}, i^{\prime}$ and $z^{\prime}$ (Fukugita et al. 1996; Smith et al. 2002). SDSS imaging are obtained using a drift-scanning mosaic CCD camera (Gunn et al. 1998) with a pixel size of $0.396^{\prime \prime}$. We downloaded the $g^{\prime}$ and $r^{\prime}$ band images (which have a median PSF of $\sim 1.4^{\prime \prime}$ in $r^{\prime}$ ) of the night sky ("corrected frames") in fits format using the Data Archive Server ${ }^{3}$. The $u^{\prime}, i^{\prime}$ and $z^{\prime}$ bands images are less sensitive and, consequently, less useful to study the profile towards the outer disk. The corrected frames, having been bias subtracted, flat-fielded, and purged of bright stars are stored at SDSS in integer format to save disk space. According to the SDSS helpdesk the pixel values get randomised appropriately before being rounded to make sure that the statistics of the background counts are what they should be. An additional offset (SOFTBIAS) of 1000 counts is added to each pixel to avoid negative pixel values and should be subtracted together with the sky value (as described in Sect. 3.3).

\footnotetext{
${ }^{3}$ http://www.sdss.org/dr2/access/index.html\#DAS
}

After inspecting all images for the 98 galaxies we had to remove 13 galaxies (marked with $a \star$ in Table 1) from further analysis (cf. Appendix B). For four galaxies (NGC 0988, NGC 4900, UGC 04684, and UGC 06162) there are one (or more) very bright foreground stars too close to the galaxy preventing any useful surface brightness measurement. Another six galaxies (NGC 0428, NGC 2712, NGC 4116, NGC 4496A, NGC 5364, and UGC 09215) are very close to the border of the SDSS field (more than $1 / 3$ of the galaxy is off the image). To avoid any problems while trying to recover the galaxies with a mosaic out of two or even three images we discarded them. Two peculiar galaxies (NGC 3023 and NGC 5218) are removed since they are clearly interacting systems. They are gravitationally highly distorted and in the case of NGC 5218 connected by an intergalactic bridge to its companion. One galaxy (PGC $032356 \equiv$ UGCA 219) turns out to be a BCD galaxy and probably even a double system. This leave 85 galaxies for the final analysis.

\subsection{Photometric calibration}

The photometric calibration is done using the $a a, k k$, and airmass coefficients (the photometric zeropoint, the extinction term and the airmass) out of the associated TsField table file for each image. From this we calculated ${ }^{4}$ our surface brightness zeropoints as: $-2.5 \times(0.4 \times[a a+k k \times$ airmass $])+2.5 \times$ $\log \left(53.907456 \times 0.396^{2}\right)$ using the pixel scale of $0.396^{\prime \prime} /$ pixel and the exposure time of $53.907456 \mathrm{~s}$ for each SDSS pixel. Our magnitudes are the conventional Pogson astronomical magnitudes in the SDSS $g^{\prime}$ and $r^{\prime} \mathrm{AB}$ system in contrast to the asinh magnitudes used in the SDSS database. The applied zero points are given in Table 2. To transform the SDSS $g^{\prime}$ and $r^{\prime}$ standard-star system magnitudes into the commonly used Johnson-Cousins $B, R$ system, we applied the transformation equations given in Smith et al. (2002): $B=g^{\prime}+0.47\left(g^{\prime}-r^{\prime}\right)+0.17$ and $R=g^{\prime}-1.14\left(g^{\prime}-r^{\prime}\right)-0.14$. For eight galaxies of the final SDSS sample we found aperture measurements in the literature. Synthetic aperture measurements on the SDSS images agree with these previous published values in the majority of cases to better than $0.1 \mathrm{mag}$. All profiles and surface brightness results, given in Table 3, are the measured values from the uncorrected SDSS data. Only at calculating mean values and plotting histograms we applied the galactic extinction correction according to Schlegel et al. (1998) using $\mu_{\text {corrected }}=\mu_{\text {measured }}-A_{r^{\prime}} / g^{\prime}$. The

\footnotetext{
${ }^{4}$ http://www.sdss.org/dr2/algorithms/fluxcal.html
} 
values we used for $A_{r^{\prime}}$ and $A_{g^{\prime}}$ are also shown in Table 3 . No attempt was made to correct the surface brightness measurements for internal extinction, since no unique recipe is available to do this. In addition, the galaxies studied here are all fairly face-on systems $\left(\langle e\rangle=0.3 \Leftrightarrow i \sim 46^{\circ}\right)$ so the expected corrections are only small. Since we are working with a local sample $(z \lesssim 0.01)$ we also do not correct for the cosmological surface brightness dimming.

\subsection{Sky subtraction}

The crucial point in using SDSS data (and in general) to study surface brightness profiles at very faint levels is the measurement of an appropriate sky value. SDSS provides in the header of each image a first estimate of such a value. However, this is just a global value which is in more than half of the cases off by more than \pm 0.2 counts $(\gtrsim 0.2 \%$ level) and therefore for our purposes not accurate enough. We need a more elaborated value depending on the size of the galaxy, thus the area of sky covered by the galaxy, and the exact position on the chip. This means that for different galaxies on the same chip we may derive different sky values.

In deep surface photometry the quality (flatness and noise) of the background around (and "below") the galaxy determines the surface brightness level down to which one can trust the profiles. A common way of measuring the sky is, after the removal of a possible large scale gradient (typically of only first order), to manually place several small boxes homogeneously around - but outside - the galaxy, avoiding foreground stars or obvious background galaxies, and measure the median of the pixel distribution in each box. The best sky value is then the mean value of all median measurements. However, the fact of storing SDSS data as integer not real numbers (cf. Sect. 3.1) hampers the use of median values in small boxes. Only by using a large number of pixels (or an inappropriate high number of boxes dealing with about 100 galaxies) one is able to recover an accurate estimate of the mean value of the background pixel distribution.

Therefore we decided to use two independent methods to determine the sky value. First, we strategically placed 2-5 large, rectangular sky boxes (of roughly $100 \mathrm{k}-200 \mathrm{k}$ pixels) as close as possible to the galaxy avoiding bright foreground stars or obvious structure in the background (e.g. from halos of very bright foreground stars) while trying to match the brightness of the sky around the galaxy. Within each box we derived the mean sky after three, $3 \sigma$, clipping iterations to remove the unavoidable contamination by faint foreground stars. The mean value of all boxes serves as the first estimate of the sky. For the second method we applied a free ellipse fit to the original image. Using the ellipse task in IRAF $^{5}$ we calculated the flux in linear steps of 10 pixels between successive ellipses forcing the program to extend the fit well beyond the galaxy with the fixed ellipticity and PA of the outer disk (see Fig. 2). By plotting the flux at these ellipses as a function of radius it becomes clear at which radial distance the ellipses leave the galaxy and enters the background, flat flux (noise), region. After visual confirmation that these ellipses are really outside the galaxy, by overplotting the ellipses on the image, the mean value and standard deviation of the fluxes within the above radius and the radius extended by $\sim 20 \%$ are used as the final sky values. In most cases the two methods agree well within \pm 0.15 counts $(\sim 0.15 \%$ of the sky). Since it is possible to automatise it, we choose the ellipse method to fix our final sky

\footnotetext{
${ }^{5}$ Image Reduction and Analysis Facility (IRAF) http://iraf.noao.edu/
}

values. They are listed in Table 2 together with a limiting surface brightness $\left(\mu_{\text {lim }}\right)$ due to a $3 \sigma$-sky error, which is used to constrain the outer boundary for the exponential fits. Our sky values can be easily inspected by downloading the public SDSS images.

To specify the error on the profiles from our sky estimate (which is much larger than the statistical errors produced by e.g. photon noise) in more detail we defined a more conservative critical surface brightness $\left(\equiv \mu_{\text {crit }}\right)$ up to where we really trust the final profile. This is placed where the profiles obtained by either over- or undersubtracting the sky by $\pm 1 \sigma$ start to deviate by more than $0.2 \mathrm{mag}$, which is at $0.54 \mathrm{mag}$ above the limiting surface brightness defined above. So the typical value for the full sample of this critical surface brightness is $\mu_{\text {crit }} \sim 27.0 r^{\prime}$-mag/ $\square^{\prime \prime}$. This is the limit down to which the slope of the outer profile could be traced confidently.

The pure background noise in the $r^{\prime}$ band images is typically slightly higher compared to the $g^{\prime}$ band image, which is expected for images taken at new moon. However, the $g^{\prime}$ band images suffer often from an electronic noise pattern in addition to the normal patchy background. Typically 6-8 more or less pronounced stripes are visible where all rows show a slightly different background level, alternating between being higher or lower. Unfortunately, this structure is not common on all images so there is no way to properly remove it by combining several images as usually done for fringe patterns. In many cases the galaxies are small compared to the strip size and the above pattern is not an issue. In other cases only small parts of the galaxies reach another strip and can be masked. However, there are some galaxies extended over two or more stripes. For these systems the sky estimate is less reliable because the contribution of each region changes with changing size of the ellipse.

For five galaxies (NGC 1042, NGC 1084, NGC 5480, NGC 5624, and PGC 006667) we had to remove at first a large scale gradient (from top to bottom) in the background of the $r^{\prime}$ band images (for NGC 1042 also in the $g^{\prime}$ band) with a linear fit (using IRAFs imsurfit).

\subsection{Data quality}

We have checked our sky estimation method comparing the profiles obtained from the SDSS images with deep surface photometry. We have used the profiles of three face-on Sbc-Sc galaxies from Pohlen et al. (2002), from which UGC 9837 is also part of our sample.

The photometrically transformed SDSS profiles match the deep $R$-band surface brightness profiles in all three cases quite well (two of the galaxies are shown in Fig. 3). The remaining differences visible are easily explained by the two very different methods applied to extract these profiles. Whereas the SDSS profiles are standard fixed ellipse fits to the images, the deep $R$-band comparison profiles are obtained by vertically averaging after polar transformation (see Pohlen et al. 2002). This test confirms that SDSS profiles can be obtained reliably down to $\sim 27.0 r^{\prime}-\mathrm{mag} / \square^{\prime \prime}$.

\subsection{Ellipse fitting and masking}

We used the IRAF task ellipse (STSDAS package) on the sky subtracted images to produce our final surface brightness profiles. We apply an extensive manual masking for each galaxy image. This is necessary to remove contamination from different sources. We masked all non-galaxy components like foreground stars, companion galaxies, or faint background galaxies. Not 


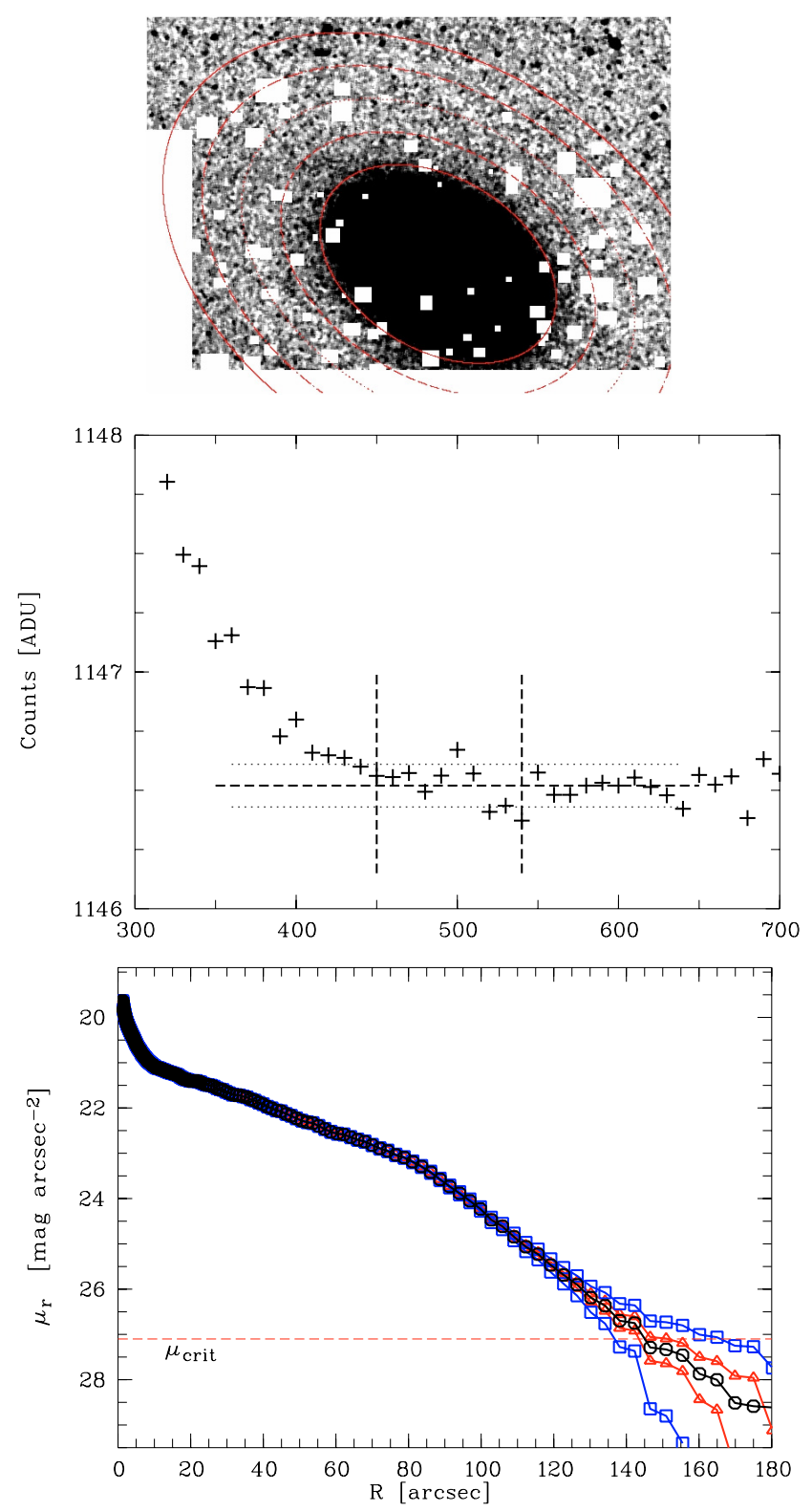

Fig. 2. Sky obtained by ellipse fitting: upper panel: $r^{\prime}$-band image of NGC 5300 exemplary overlayed by eight fixed ellipses at every 100 pixels in the range 300-700 pixels. The white rectangular regions are masked areas. Middle panel: mean isophotal intensity (in counts) of the fixed ellipses fitted every 10 pixels. The region between the vertical dashed lines is used to determine the sky value (1146.52 \pm 0.09$)$, which is indicated as a horizontal dashed line together with the two dotted lines at $\pm 1 \sigma$. Lower panel: final surface brightness profile (circles) overlayed by the four profiles obtained by either over- or undersubtracting the sky by $\pm 1 \sigma$ (triangles) or by $\pm 3 \sigma$ (squares). The horizontal dashed line gives the critical surface brightness $\left(\mu_{\text {crit }}\right)$ up to where we trust the profile i.e. where the $\pm 1 \sigma$ profiles deviate by more than $0.2 \mathrm{mag}$.

masked are extended spiral arms, or outer asymmetries clearly belonging to the galaxy itself. The masking is primarily done in a $9 \times 9$ pixel $\left(3.6^{\prime \prime} \times 3.6^{\prime \prime}\right)$ median smoothed version of the image, ideally suited to visualise structure in the background. This step also ensures to reliably trace the halos of the foreground stars to large radii. Whereas this is necessary for bright stars it is a conservative measure for fainter ones. In a second round any remaining stars in the unsmoothed image are also masked.

The common center for each concentric ellipse is fixed by a Gaussian fit to the bright nucleus of the galaxy. For 21 galaxies
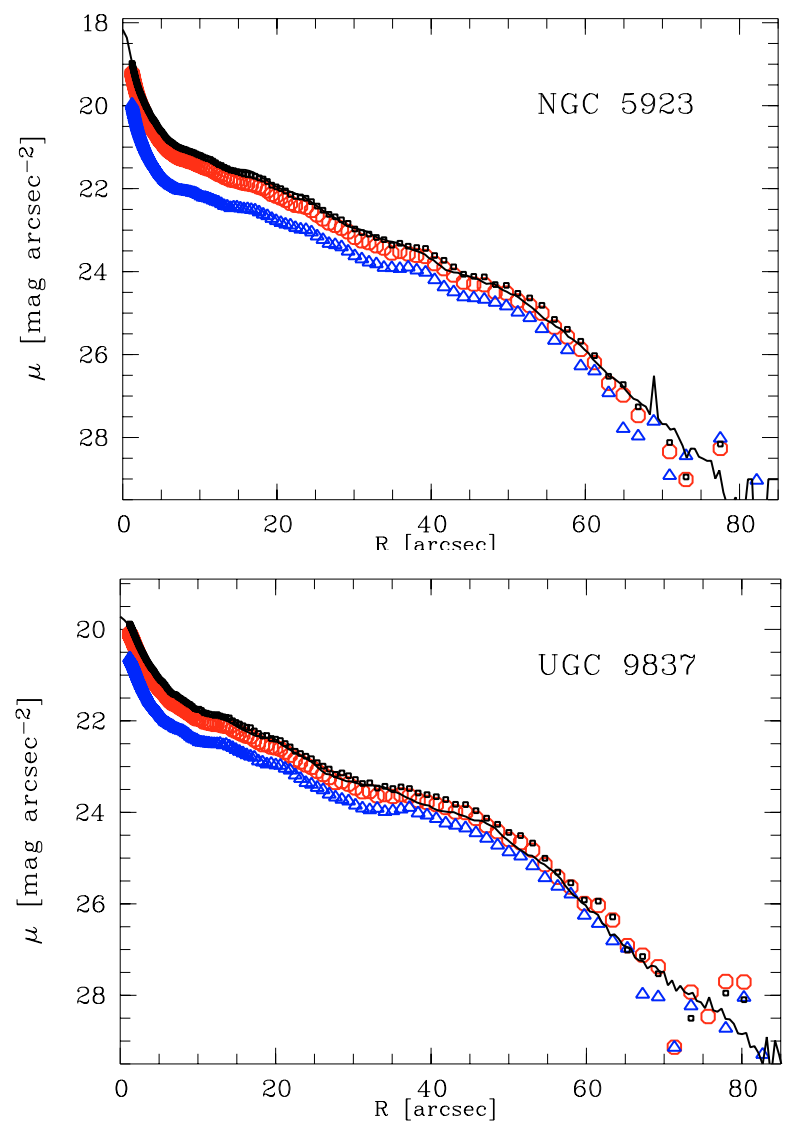

Fig. 3. SDSS quality check: azimuthally averaged, radial surface brightness profiles obtained from SDSS images ( $r^{\prime}$ band with big circles; $g^{\prime}$ band with big triangles)) are compared with much deeper imaging (thin, continuous line) presented in Pohlen et al. (2002). The small squares are obtained by using the transformation to convert SDSS $g^{\prime}$ and $r^{\prime}$ into standard Johnson $R$ band (used by Pohlen et al. 2002) following Smith et al. (2002).

(marked in Table 2 with a $\dagger$ ) the disk is lopsided or there is no bright center visible so we first allowed the ellipse centers to vary, choosing the best center representative for the outer disk.

We used a logarithmic radial sampling with steps of 0.03 to increase the $\mathrm{S} / \mathrm{N}$ especially in the outer parts at cost of larger radial bins compared to linear sampling. Iterative $3 \sigma$ rejection along the ellipse is applied to minimise the influence of cosmic rays or any remaining faint foreground stars.

We fitted two different sets of ellipses (three in case of a necessary free center fit) to each galaxy image (for general information on ellipse fitting see Erwin et al. 2006). The first, free ellipse fit tends to follow morphological features like bars, spirals, or asymmetries so it is not ideal to characterise the outer, underlying disk component, which we want to address in this work. Therefore we used a fixed ellipse fit to produce our final surface brightness profiles which will be the only ones discussed in the following sections. Fixing a single ellipticity and position angle for a galaxy is based on the assumption that the disk is axisymmetric and round and the ellipticity and PA values are representative for the orientation (inclination angle) of the galaxy.

The initial free ellipse fit (fixed center, free ellipticity and PA) is used to determine the best set of ellipticity and PA describing the outer disk. These values are typically taken at the radius where the mean flux of the best fitted free ellipse reaches the value of the standard deviation of our background measurement in large boxes ( $1 \sigma$ criterion). This limit ensures enough 


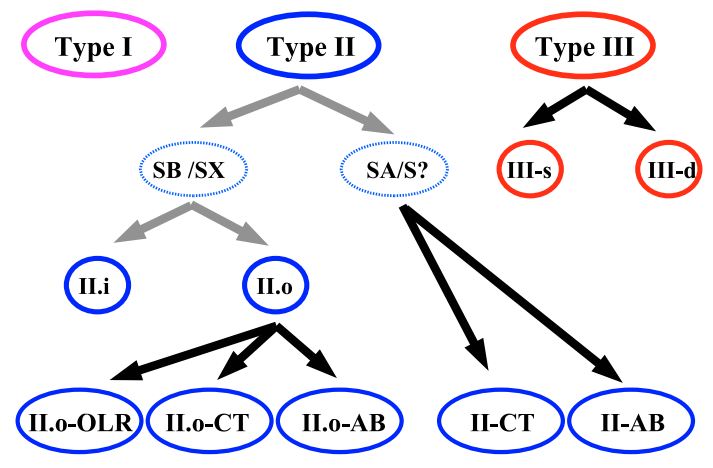

Fig. 4. Classification schema (following Erwin et al. 2006): The three main disk types according to break features in their surface brightness profiles are Type I (no break), Type II (downbending break), and Type III (upbending break). In case the galaxy is barred (SB/SX) the downbending breaks (Type II) are subdivided according to the position of the break in respect to the bar: Type II.i (inside) or Type II.o (outside). An additional level of subclassification is applied for this class trying to relate the observed break to a physical origin. These are Type II.o-AB, Type II.o-OLR, and Type II.o-CT (see text). For apparently unbarred galaxies (SA/S?) we use only Type II-CT or Type II-AB. The upbending breaks (Type III) are subdivided according to the possible nature of the outer region: (d)isk- or (s)pheroid-like. Some galaxies are better described with two breaks, each of which could be associated to one of the individual types. They are assigned a mixed classification (e.g. Type IICT + III).

$\mathrm{S} / \mathrm{N}$ to fit a free ellipse but is small enough to be in the radial region dominated by the outer disk. Ideally one would like to determine the ellipticity and PA of the outer disk not photometrically, but by means of kinematical informations. Since this is not possible for the whole sample we used only the photometric way to get a set of homogeneous values for all our galaxies. However, for some galaxies the $1 \sigma$ criterion clearly marked a radius in a region with rapidly changing ellipticity and PA values, or a region which is not representative of the outer disk. In this cases we chose, after visual inspection, a different radius to represent the disk, which is typically the outermost successfully fitted free ellipse.

The $r^{\prime}$ band and $g^{\prime}$ band ellipticity and PA values are determined independently. Since a galaxy should have only a single pair of PA and ellipticity (depending on the inclination and intrinsic circularity) independent of the wavelength used, we averaged the two values in case of no major problem in one of the bands. The fixed ellipse fits to the $r^{\prime}$ and $g^{\prime}$ band is therefore repeated using these mean values (see Table 2) and the resulting profiles are shown in Appendix A.

\section{Analysis}

\subsection{Classification}

We have classified (following Erwin et al. 2006) each profile according to observed break feature using the following nomenclature: Type I (no break), Type II (downbending break), and Type III (upbending break). A schematic view of our classification is shown in Fig. 4. Images and profiles for some typical examples of each class are given in Fig. 5.

The Type I classification follows the nomenclature established by Freeman (1970). Aside from a varying bulge component these galaxies exhibit a region well described by a single exponential allowing for some wiggles associated with substructure in the disk such as spiral arms or prominent star forming regions.
The galaxies displaying a break with a downbending steeper outer region could be labelled Type II as (Freeman 1970) originally proposed. Although he states at some point the inner deficit is not far from the center of the system there is no further restriction on this distance. In addition, for the three most prominent Type II galaxies in his sample he states that the outer exponential disk begins outside the main region of spiral-arm activity, so clearly outside a central region.

Following Erwin et al. (2006) the Type II class is subdivided into two groups in case the galaxy is barred. A Type II.i (inside) and a Type II.o (outside) depending on the position of the break in respect to the bar length (inside or outside the bar region). Moreover, we tried to subclassify the Type II and Type II.o profiles even further into additional three groups, which now tend to categorise the observed profiles according to their potential physical origin. First, profiles where the apparent break can be associated with large scale asymmetries of the galaxy in itself (only present in Sc-Sd galaxies). These are either associated to a lopsided disk having a well defined center, which is different from the applied ellipse centered on the outer disk, or to some large scale asymmetries in (or beyond) the main body of the outer disk (cf. extended spiral arms). These profiles are called Type II-AB, for Apparent (or Asymmetric) Break. Another group, showing the break radius at around 2-2.5 times the bar radius, is probably related to a resonance of the bar as described in Erwin et al. (2006). The bar itself is most often nicely marked by the presence of an additional inner ring. These profiles are classified as Type II.o-OLR, for Outer Lindblad Resonance which happens to be at the position of $\sim 2$ times the bar radius. The remaining profiles revealing a broken exponential behaviour are called Type II-CT, since they are best associated to what we call now Classical Truncations discovered by van der Kruit (1979). Three nice examples of unbarred SbcSc face-on galaxies are presented by Pohlen et al. (2002). For Type II-CT galaxies with bars we made sure that the break is significantly further out than typical for the Type II.o-OLR breaks.

The galaxies with a break followed by an upbending (shallower outer) profile are called Type III according to Erwin et al. (2005a) who find two sub-classes. One (Type III-s) showing a fairly gradual transition and outer isophotes that are progressively rounder than the isophotes in the main disk, suggesting a disk embedded within a more spheroidal outer zone associated to a halo or an extended bulge component. The other type (Type III-d) exhibits a rather sharp transition with the outer isophotes being not significantly rounder. Whereas measuring the ellipticity is an appropriate way to disentangle for early-type galaxies, devoid of prominent spiral arm structure misleading the free ellipse fits, it will most probably fail for late-types. So we decided here to classify them all as Type III only subclassifying those with visible spiral arm structure beyond the break radius, obviously associated with an outer disk-like region, as Type III-d (cf. Appendix A). In our subsequent paper we will study if colour variations at and beyond the break could potentially be used to decide between these subclasses. The colour profile of a transition between a star-forming inner disk and a halo/envelope (Type III-s) may look different from a transition between an intermediate-age inner disk to a predominantly younger outer disk (Type III-d).

Some galaxies have a more complex surface brightness distribution. They are better described with two breaks each of which could be associated to an individual type (as described above). These are called mixed classification (see Fig. 5). For all the galaxies with a break in the profile we verified that changes 

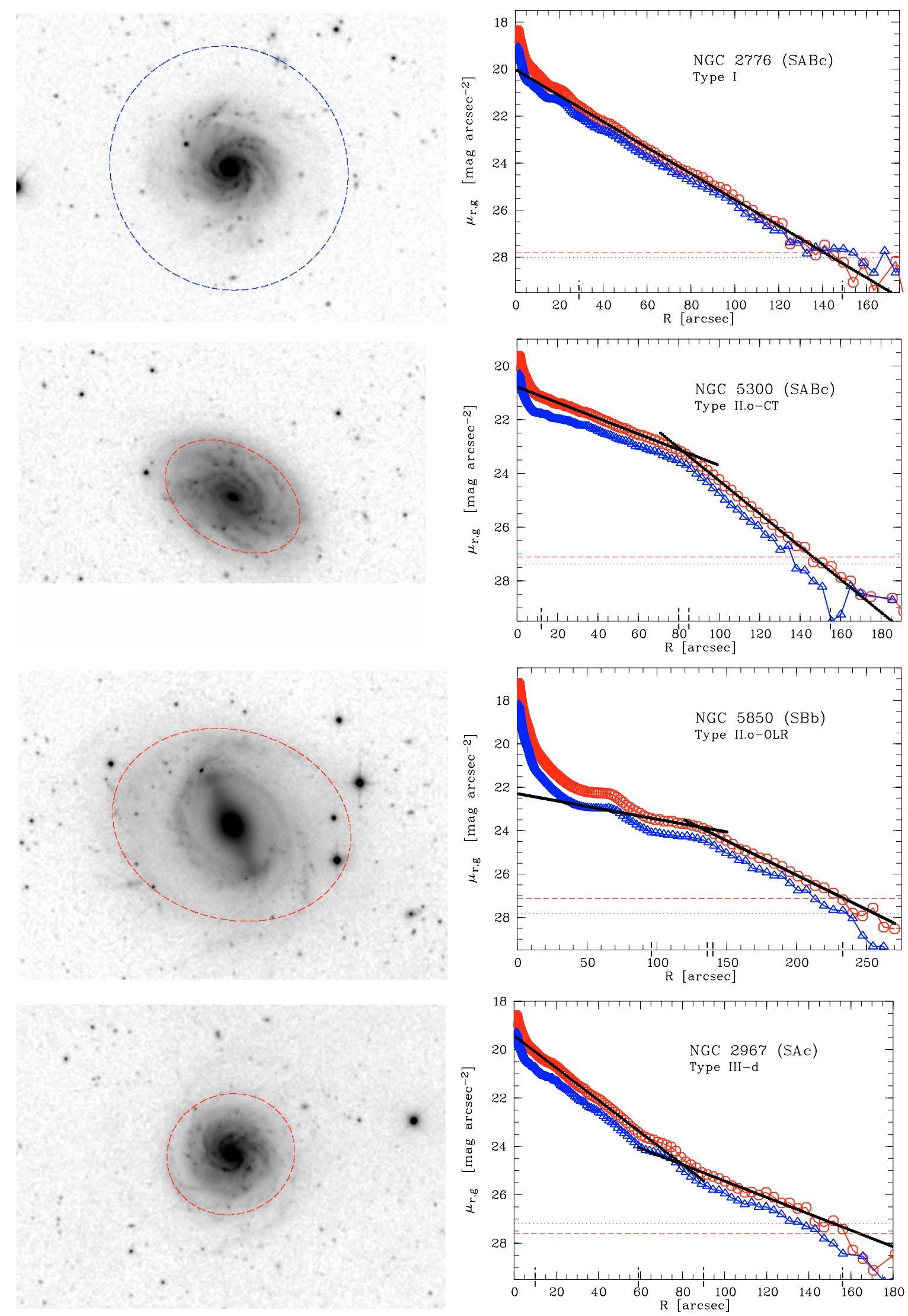

Fig. 5. Prototypical examples for each class of profiles: Type I, Type II-CT, Type II.o-OLR, and Type III (top to bottom). Left panels: $r^{\prime}$-band images (unrotated cut-outs from the SDSS fields) with the break radius marked with an ellipse. The ellipse for the first Type I galaxy corresponds to the noise limit at $\sim 140^{\prime \prime}$. Right panels: azimuthally averaged, radial SDSS surface brightness profiles in the $g^{\prime}$ (triangles) and $r^{\prime}$ (circles) band overlayed by $r^{\prime}$ band exponential fits to the individual regions: single disk or inner and outer disk. In addition we show the critical surface brightness $\left(\mu_{\text {crit }}\right)$ for each band (dotted and dashed horizontal lines), down to which the profile is reliable (see Appendix A for a detailed legend). 

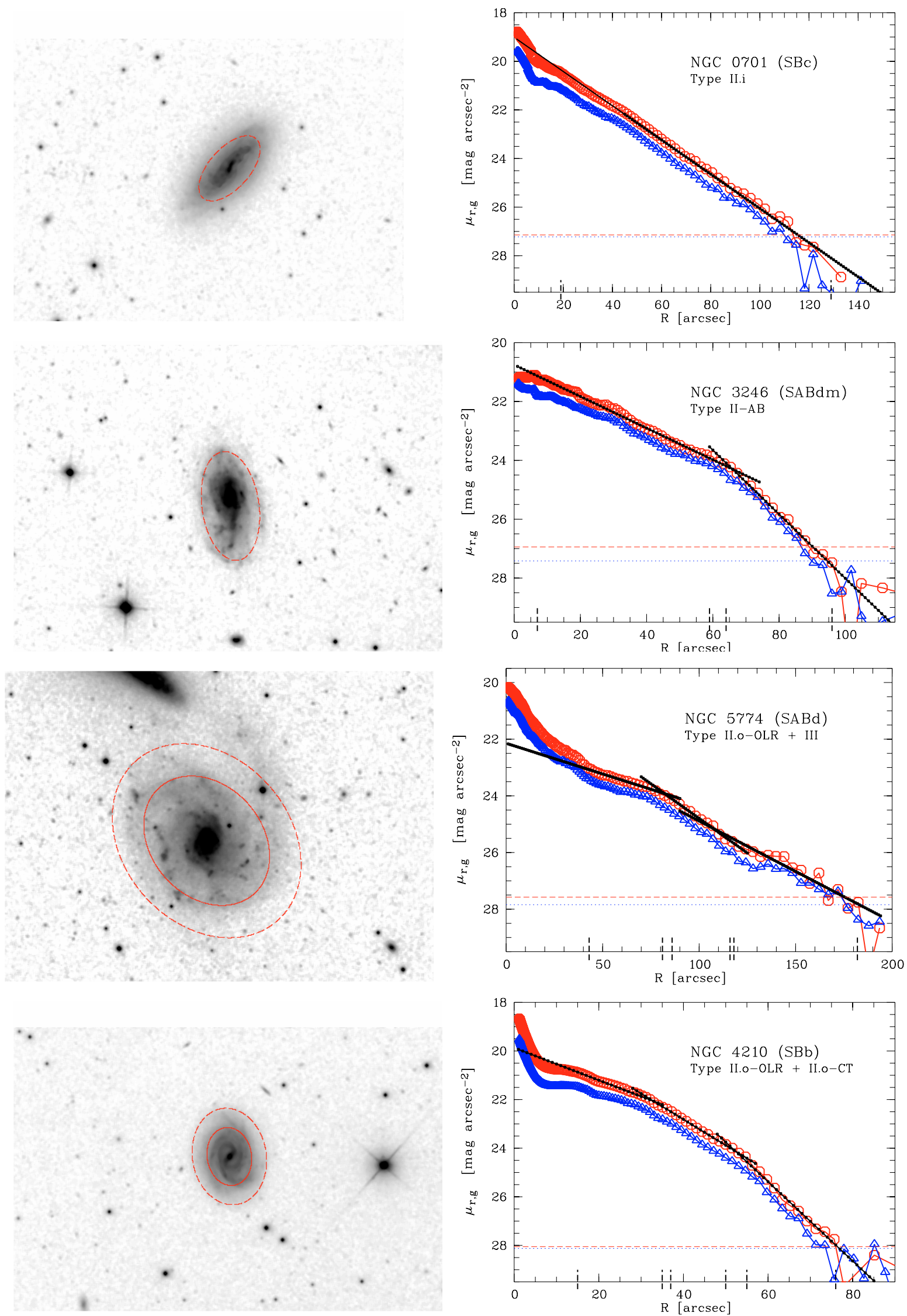

Fig. 5. continued. The ellipse for the first Type II.i galaxy corresponds to the inner boundary at $\sim 20^{\prime}$. For the mixed classifications two ellipses are shown corresponding to the associated breaks. 
in the sky value (e.g. subtracting $\pm 3 \sigma$ to the sky level) will not produce an untruncated Type I profile (see Fig. 2).

Please note that our classification Type I and Type II are used slightly different from MacArthur et al. (2003). Our Type II-CT class is called Type I with truncation in their study and their Type II is a mix of our Type II.i and all other Type II.o classes. Since we do not have infrared images we cannot comment on their "transition" class. However, we note that for IC 1067 (see Appendix A for a detailed discussion) the $r^{\prime}$-band profile is barely consistent with being Type I whereas in the $g^{\prime}$-band it is clearly a Type II.o-OLR which is consistent with the profile shown by Erwin et al. (2006). This might imply that in very few cases dust is playing a role in shaping the observed profile close to the center. So this profile could be a Type $\mathrm{I}$ in the $K$-band, which has to be investigated in further studies.

\subsection{Deriving break radius, break region and scalelengths}

In addition to our pure identification - break (down- or upbending) yes or no - we have quantified the radial distance $R_{\text {br }}$ where the break occurs, characterised its sharpness and derived an inner and outer scalelength $\left(h_{\text {in }}, h_{\text {out }}\right)$ (see Fig. 6).

To determine the scalelengths we restricted our fits to the 1D, azimuthally averaged, surface brightness profiles obtained with fixed ellipse fits (cf. Sect. 3.5). Since a detailed bulge to disk decomposition is beyond the scope of this paper, we used the so called "marking the disk" method by applying boundaries and fitting simple exponential functions: $\mu(R)=\mu_{0}+1.086 \times R / h$. The inner boundary $\left(b_{1}\right)$ is chosen manually to exclude the region obviously dominated by the bulge plus bar (shoulder) component. The outer boundary $\left(b_{4}\right)$ is taken where the surface brightness profile reaches $(3 \sigma)$ of the noise level obtained from the sky ellipse fitting described in Sect. 3.3.

If the profile shows a broken exponential we need an additional boundary marking the break. This could be placed by eye, but to obtain the break radius objectively we used the following approach. At first an approximated derivative of the profile is calculated at each point, by fitting a straight line including four points around each radius. This provides a measure of a local scalelength $\left(h_{\text {local }}\right)$ along the radial axis (cf. Fig. 6). To get a linear spacing of surface brightness measurements along the radial axis and to decrease the noise in the $h_{\text {local }}$ distribution we first rebinned the profile to linear units using a spline function and then median smoothed the $h_{\text {local }}(R)$ profile adapting the smoothing length to $5 \%$ of the full extent of the profile.

Our general assumption is now that we have two regions with nearly constant scalelength together with a change (up- or downbending) in the profile. This will be reflected by two plateaus in the plot of $h_{\text {local }}(R)$ (see Fig. 6). Depending on the actual shape (e.g. sharpness) of the transition region $h_{\text {local }}(R)$ will move from one scalelength region around $h_{\text {in }}$ to another region around $h_{\text {out }}$. To quantify a single radius defining this break we used then the radius where the $h_{\text {local }}(R)$ profile crosses the horizontal line set by a characteristic value. In case the two regions do not cover the same radial range, the characteristic value is always taken as the median of the $1 / h_{\text {local }}$ distribution, to avoid a bias towards the more extended region.

To characterise the shape of the break we defined an additional region around the break. Ideally a sharp break would translate into a step function in the $h_{\text {local }}(R)$ profile whereas a smooth transition would cross the line set by the weighted mean value with a finite slope. Therefore we defined an inner and outer boundary of the break region where the profile, starting from the break, reaches a value within two standard deviations away from the mean calculated for the inner/outer region separately (cf. Fig. 6). The derived break radii are almost always consistent with those derived by eye, but of course fail if one of the regions is very small (e.g. in the case of IC 1067, see Appendix A) or get uncertain if more than two regions with roughly constant scalelengths are involved (as for the mixed classifications, see below and Sect. 5).

Using the boundaries of the break region $\left(b_{2}\right.$ and $\left.b_{3}\right)$ to mark the separation of an inner and outer exponential disk, the final scalelengths are derived from two simple exponential fits $\left(\mu(R)=\mu_{0, \text { in }}+1.086 \times R / h_{\text {in }}\right.$ and $\left.\mu(R)=\mu_{0, \text { out }}+1.086 \times R / h_{\text {out }}\right)$ to the original profile. To decrease the noise we give priority to this classical approach to determine the scalelength over using a weighted mean from the $h_{\text {local }}$ values inside the now defined inner and outer region. Fitting the two exponentials one could alternatively define the break radius, as the meeting point of the two exponential fits. However, since this would add an influence by the actual shape of the two disk regions into the positioning of the break radius we decided against it.

In cases where the derivative profile $\left(h_{\text {local }}(R)\right)$ crosses the horizontal line set by the characteristic value twice (three or more times is considered to be explained by wiggles caused by asymmetries such as spiral arms) we check if we could apply a mixed classification. This implies assigning two breaks associated to the individual types as discussed in Sect. 4.1 which significantly improve the overall fit. The boundaries for both breaks are then used to fit three exponential regions.

The final results for all the galaxies are given in Table 3 . We do not provide individual errors for the fitting parameters in Table 3, since their uncertainty is a complex combination of random and systematic errors. Typical random errors of the exponential fitting routine alone are $2 \%$ for $h_{\text {in }}, 3 \%$ for $h_{\text {out }}$, \pm 0.04 mag for $\mu_{0 \text {,in }}$, and \pm 0.17 mag for $\mu_{0, \text { out }}$. The uncertainty in the sky subtraction, however, translates to a clearly systematic error. In the case of over/under-subtraction the measured scalelengths are systematically smaller/larger and the central surface brightnesses are systematically lower/higher. To estimate how the error on the sky subtraction alone effects our parameters we have over/under-subtracted the measured sky level by $\pm 1 \sigma$. Typical errors are then only $\pm 1 \%$ for $h_{\text {in }}$ and \pm 0.02 mag for $\mu_{0 \text {,in }}$, but $\pm 7 \%$ for $h_{\text {out }}$ and \pm 0.38 mag for $\mu_{0 \text {,out }}$. Another source of uncertainty is generated by the positioning of the boundaries $\left(b_{1-4}\right)$. This uncertainty can not be easily quantified since it depends on the shape (up/down-bending) of, and features (bulges, bars, etc.) in, the surface brightness profiles and cause systematic errors. To minimise their contribution in our determination of the structural parameters each fit is verified by eye.

\section{Results}

Our final classifications are presented in Table 3 and discussed in more detail individually in Appendix A. For four galaxies in common with Erwin et al. (2006) the classification (as well as the final profiles) agree very well. All features in the surface brightness profiles classified here are always consistently found in deep surface photometry profiles available (cf. Appendix A) in the literature (e.g. Courteau 1996).

About $90 \%$ of our sample can be classified into one of the classes described in Sect. 4.1. However, the remaining galaxies are better described with two breaks in their surface brightness profiles. Galaxies with extra breaks are classified as mixed. In almost all cases this is a combination of a downbending break, either Type II-CT, Type II.o-OLR, Type II-AB, or Type II.i, classified as described in Sect.4.1, followed by an additional 

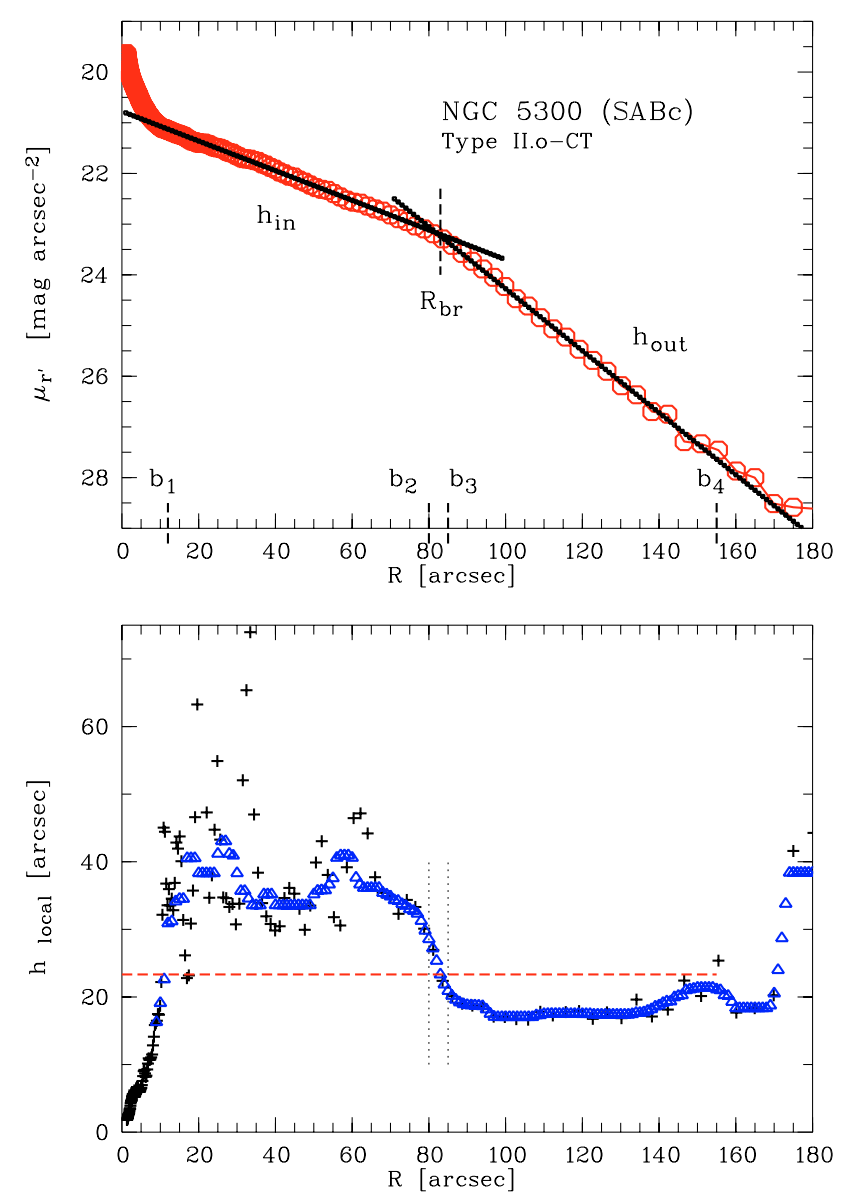

Fig. 6. Example of how to establish the break radius, the break region and the associated scalelengths: upper panel: azimuthally averaged, surface brightness profile, overlayed by the best fitting exponentials (with scalelengths $h_{\text {in }}$ and $h_{\text {out }}$ ) marking the position of the boundaries $\left(b_{1-4}\right)$ and the break radius $R_{\mathrm{br}}$. Lower panel: approximated derivative (crosses) of the profile providing a local scalelength $\left(h_{\text {local }}\right)$ together with the rebinned and smoothed version (triangles). The horizontal dashed line marks our characteristic (or mean) value which is the median of the $1 / h_{\text {local }}$ distribution. The vertical dotted lines mark the inner and outer boundary of the break region.

upbending break in the very outer parts. These galaxies are classified as e.g. Type II.o-OLR + III. Interestingly, none of the Type III profiles show an additional outer truncation within our sensitivity limit.

Only two galaxies (see detailed explanation in Appendix A) exhibit an inner downbending break followed by an additional outer downbending break. In one case (NGC 4517A) the outer sharp drop is clearly associated to a Type II-AB, whereas the inner break is consistent with being a Type II.o-OLR or Type IICT. The other case is NGC 4210, where the inner boundary of an extended, exponential, break region is consistent with a Type II.o-OLR break leaving the outer break to be Type II-CT. This galaxy could be the ideal test object to study the difference between Type II.o-OLR and Type II-CT breaks. Finally, for only one galaxy, IC 1067, the classification in the $r^{\prime}$ and $g^{\prime}$-band could be different (see Sect. 4.1).

\subsection{Frequencies}

The vast majority (almost 90\%, see Table 4) of the galaxies exhibit surface brightness profiles with breaks, only $9(11 \pm 4 \%)$
Table 4. Frequency of disk types: listed are the number of galaxies per profile type. The values in brackets are those associated to more than one break type (mixed classification). Therefore the last column gives the frequency for each break type and does not add up to $100 \%$.

\begin{tabular}{lcr}
\hline \hline Profile type & Number [\#] & Frequency [\%] \\
\hline Type I & 9 & $11 \pm 3$ \\
Type II & 56 & $66 \pm 5$ \\
- Type II.i & $4(1)$ & $6 \pm 3$ \\
- Type II-CT & $28(2)$ & $33 \pm 5$ \\
- Type II.o-OLR & $13(5)$ & $15 \pm 4$ \\
- Type II-AB & $11(3)$ & $13 \pm 4$ \\
Type III & $28(7)$ & $33 \pm 5$ \\
\hline
\end{tabular}

are reasonably well described (allowing sometimes for quite extensive deviations) as being purely single-exponential (Type I). Even adding the barred galaxies showing only a dip inside the bar radius (Type II.i), their frequency increases up to merely $15 \pm 4 \%$.

$66 \pm 5 \%$ of the galaxies are classified as Type II with a break and downbending profiles (61\% excluding Type II.i). The frequencies of the three main subgroups are the following: a) $33 \pm 5 \%$ (from the full sample) exhibit a classical truncations (Type II-CT); b) $15 \pm 4 \%$ (from the full sample) are classified as having breaks with downbending profiles that could be (size wise) related to the presence of the bar (Type II.o-OLR); and c) $13 \pm 4 \%$ (from the full sample) show breaks in their profile (Type II-AB) that could be originated by a lopsided or asymmetric disk, not allowing us to probe for a real, intrinsic break in the stellar light distribution using our azimuthally averaged, fixed ellipse fits.

$33 \pm 5 \%$ of the galaxies show a break with an upbending profile (Type III).

\subsection{Parameter distribution}

In the following we give all measurement of lengths and ratios as obtained for the $r^{\prime}$ band and excluding the mixed classifications if not otherwise stated.

\section{No breaks (Type I)}

The mean central surface brightness and scalelength of the 9 Type I galaxies are $\mu_{0}=20.2 \pm 0.8 r^{\prime}-\mathrm{mag} / \square^{\prime \prime}$ and $h=$ $2.8 \pm 0.8 \mathrm{kpc}$ respectively, which are typical values for local galaxies (cf. MacArthur et al. 2003; de Jong 1996). We confidently trace the profiles down to our critical surface brightness of $\mu_{\text {crit }} \sim 27.0 r^{\prime}$-mag/ $\square^{\prime \prime}$ (see Fig. 2). So, in terms of scalelength we do not find a break down to 6-8 times the measured scalelength.

\section{Classical truncations (Type II-CT)}

The break in the surface brightness profiles appears at $9.2 \pm$ $2.4 \mathrm{kpc}$ and ranges between $5.1 \mathrm{kpc}$ and $14.7 \mathrm{kpc}$ with a slight trend towards galaxies with higher luminosities having larger break radii (cf. Fig. 7). We do not find a systematic difference between the break radii as measured in the $r^{\prime}$ or $g^{\prime}$ band. In relative units the break is at $2.5 \pm 0.6$ times the inner scalelength with values between 1.4 and 4.2 , uncorrelated with rotational velocity and with a weak trend towards higher values for brighter galaxies (cf. Fig. 8). The value found here for the position of the break in terms of radial scalelength is apparently smaller (although 

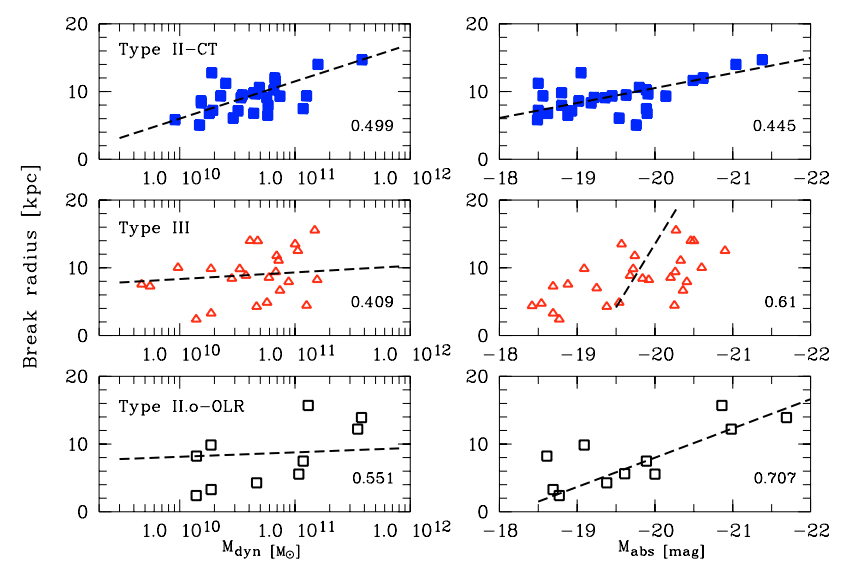

Fig. 7. Break radius $\left(R_{\mathrm{br}}\right)$ in absolute units $(\mathrm{kpc})$ versus dynamical mass $\left(\mathcal{M}_{\text {dyn }}=2.326 \times 10^{5} \frac{D_{25}}{2} v_{\text {rot }}{ }^{2}\right)$ and absolute magnitude $\left(M_{\text {abs }}\right)$ in the $B$-band according to LEDA for the different break types: Type II-CT (upper row), Type III (middle row), and Type II.o-OLR (bottom row). Overplotted are robust linear fits (dashed lines) to guide the eye and in the lower right corner of each plot the Spearman rank correlation coefficient. The break radius correlates in all three cases with absolute magnitude. With the exception of the Type II-CT, the relation of the position of the break with the dynamical mass is less tight.
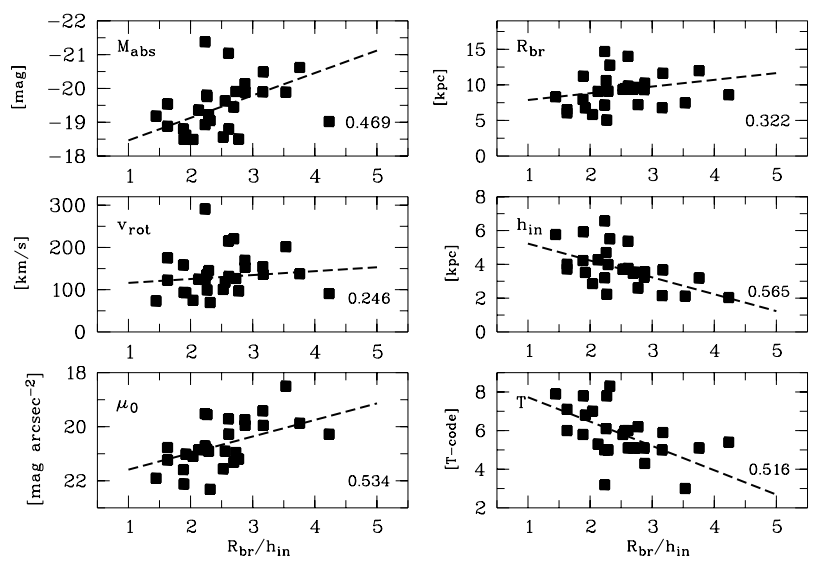

Fig. 8. Classical truncations (Type II-CT): break radius in units of inner scalelength versus absolute magnitude $\left(M_{\mathrm{abs}}\right)$ (left, upper panel), rotational velocity $\left(v_{\text {rot }}\right)$ (left, middle panel), extinction corrected central surface brightness of the inner disk $\left(\mu_{0, \text { in }}\right)$ (left, lower panel), break radius in linear unit $\left(R_{\mathrm{br}}\right)$ (right, upper panel), inner scalelength in linear unit $\left(h_{\text {in }}\right)$ (right, middle panel), and Hubble type $(T)$ (right, lower panel). Overplotted are robust linear fits (dashed lines) to guide the eye and in the lower right corner of each plot the Spearman rank correlation coefficient.

compatible within $1 \sigma$ ) to the one quoted for three galaxies by Pohlen et al. (2002): 3.9 \pm 0.7 . According to Pohlen et al. (2002), the one galaxy in common (UGC 9837) has a value of 3.1 for $R_{\mathrm{br}} / h_{\text {in }}$, which is consistent with our ratio of 2.7 obtained from the SDSS image. The two galaxies with high values (4.3 and 4.2) are too distant to be in our sample and both are intrinsically large, having break radii of $14.7 \mathrm{kpc}$ and $21.1 \mathrm{kpc}$. Fitting the SDSS profile (converted to Johnson $R$ according to Sect. 3.2, see Fig. 3) and using our method for one of them (NGC 5923), we obtain also a consistent value (4.1 compared to 4.3 quoted by Pohlen et al. 2002). Due to the completely different masking of a very close extended companion the comparison for the third galaxy (NGC 5434) is not straightforward (3.2 compared to 4.2). So neither their larger intrinsic size nor a systematic error in determining the ratio $\left(R_{\mathrm{br}} / h_{\mathrm{in}}\right)$ is responsible for the apparent

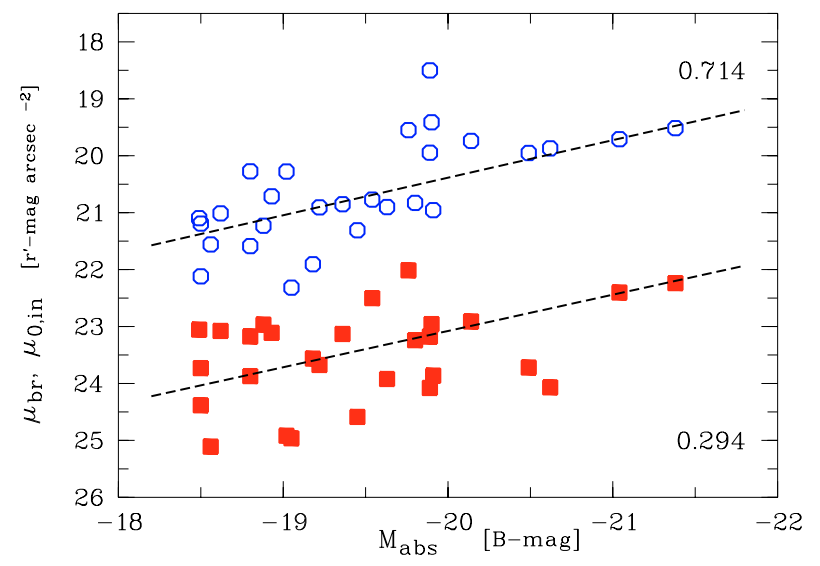

Fig. 9. Classical truncations (Type II-CT): extrapolated central surface brightness $\mu_{0}$ (open circles) and surface brightness at the break radius $\mu_{\mathrm{br}}$ (filled squares) in the $r^{\prime}$ band versus absolute magnitude $\left(M_{\mathrm{abs}}\right)$ in $B$ according to LEDA. Overplotted are robust linear fits (dashed lines) to guide the eye and in the lower and upper right corner the Spearman rank correlation coefficient for each data set.

mismatch of the mean values for the two samples, suggesting that its origin is due to small number statistics.

However, our mean value of $2.5 \pm 0.6$ is significantly lower compared to the $4.5 \pm 1.0$ value obtained for 16 face-on galaxies by van der Kruit (1988). This large offset is almost certainly due to the different definition (break versus cut-off) and method used to mark the truncation. We determine the truncation at the position of the measured break in the profile (so quite far in), whereas van der Kruit was looking for a sharp outer boundary estimated from isophote maps. Our value is supported by Bosma \& Freeman (1993) who stated that for 7 galaxies (with breaks in their surface brightness profiles), of the 21 reported by Wevers (1984) (so virtually the same dataset van der Kruit 1988 used), the mean value of the break to scalelength is $2.8 \pm 0.4$, so very close to our mean value.

The mean scalelength of the inner disk $h_{\text {in }}$ in our sample is $3.8 \pm 1.2 \mathrm{kpc}$ and ranges between $2.0 \mathrm{kpc}$ and $6.6 \mathrm{kpc}$, which is typical for local galaxies (cf. MacArthur et al. 2003; de Jong 1996).

The central surface brightness (extrapolated from the fitted inner exponential, corrected for galactic extinction, but uncorrected for inclination) ranges between $\mu_{0, \text { in }}=18.5 r^{\prime}-\mathrm{mag} / \square^{\prime \prime}$ and $\mu_{0, \text { in }}=22.3 r^{\prime}-\mathrm{mag} / \square^{\prime \prime}\left(18.9-22.8 g^{\prime}-\mathrm{mag} / \square^{\prime \prime}\right)$ with a clear trend towards galaxies with fainter luminosities having a fainter central surface brightness (cf. Fig. 9) as seen by MacArthur et al. (2004) or de Jong (1996).

However, the surface brightness at the break radius only weakly correlates with absolute magnitude (cf. Fig. 9) and peaks (cf. Fig. 10) at a mean value of $\mu_{\mathrm{br}}=23.5 \pm 0.8 r^{\prime}$-mag/ $\square^{\prime \prime}$ $\left(\mu_{\mathrm{br}}=23.8 \pm 0.8 g^{\prime}-\mathrm{mag} / \square^{\prime \prime}\right)$. The mean ratio of inner to outer scalelength is $h_{\text {in }} / h_{\text {out }}=2.1 \pm 0.5$ with a mildly peaked distribution ranging between $h_{\text {in }} / h_{\text {out }}=1.3-3.6$.

\section{OLR breaks (Type II.o-OLR)}

The break radii for the Type II.o-OLR breaks span a wider range, compared to Type II-CT breaks, between $2.4 \mathrm{kpc}$ and $25.0 \mathrm{kpc}$ (mean: $9.5 \pm 6.5 \mathrm{kpc}$ ) again with a clear trend towards galaxies with higher luminosities having larger break radii (cf. Fig. 7). In terms of inner scalelength almost all breaks appear in the small range between 1.4-2.4 times $h_{\text {in }}$ (with a mean at $1.7 h_{\text {in }}$ ), except for one galaxy (NGC 5430) where the break is at $4.4 h_{\text {in }}$ 


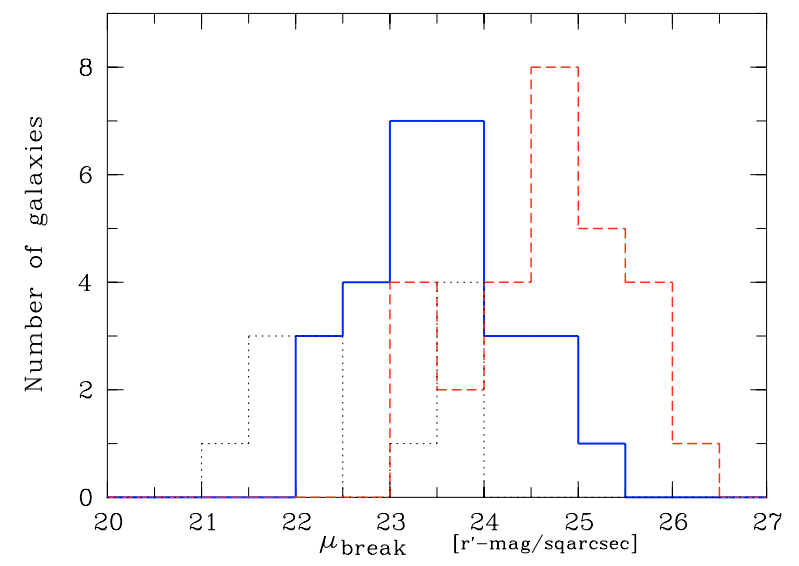

Fig. 10. Histogram of the surface brightness (in the $r^{\prime}$ band) at the break radius for the galaxies of Type II-CT (solid line), Type III (dashed line), and Type II.o-OLR (dotted line).

but which could be also classified as Type I with bumps shaped by the prominent bar (cf. Appendix A). The surface brightness at the break radius is $\mu_{\mathrm{br}}=22.6 \pm 1.0 r^{\prime}-\mathrm{mag} / \square^{\prime \prime}$. Although on the lower end compared to the distribution for $R_{\mathrm{br}} / h_{\mathrm{in}}$ and $\mu_{\mathrm{br}}$, neither of these parameters works out as a clear discriminatory property for the two different downbending break types Type IICT and Type II.o-OLR.

\section{Upbending profiles (Type III)}

In relative units the Type III break appears further out, compared to Type II-CT or Type II.o-OLR breaks, at $4.9 \pm 0.6$ times the inner scalelength with values between 3.7 and 5.8 (excluding the seven galaxies with mixed classifications where an inner scalelength is not well defined). However, in absolute units they span the same range. The break appears at $9.3 \pm 3.3 \mathrm{kpc}$ and ranges between $4.4 \mathrm{kpc}$ and $15.5 \mathrm{kpc}$ having the same trend with luminosity (cf. Fig. 7). The inner scalelength is on average smaller with $1.9 \pm 0.6 \mathrm{kpc}$ ranging only between $0.9 \mathrm{kpc}$ and $3.0 \mathrm{kpc}$. The central surface brightness (extrapolated from the fitted inner exponential and corrected for galactic extinction) ranges only between $\mu_{0, \text { in }}=18.1 r^{\prime}-\mathrm{mag} / \square^{\prime \prime}$ and $\mu_{0, \text { in }}=20.3 r^{\prime}-\mathrm{mag} / \square^{\prime \prime}$ (mean: $19.2 \pm 0.6 r^{\prime}-\mathrm{mag} / \square^{\prime \prime}$ ) without showing the clear trend with luminosity as for the Type II-CT breaks. The mean value for the surface brightness at the break radius, $\mu_{\mathrm{br}}=24.7 \pm 0.8 r^{\prime}$ $\mathrm{mag} / \square^{\prime \prime}\left(\mu_{\mathrm{br}}=25.6 \pm 0.4 r^{\prime}-\mathrm{mag} / \square^{\prime \prime}\right.$ for the seven galaxies with additional downbending breaks inside), is clearly fainter compared to the Type II-CT breaks (see Fig. 10).

\subsection{Correlations}

Plotting the frequency of break types versus the Hubble types (grouped in three bins: $\mathrm{Sb}-\mathrm{Sbc}, \mathrm{Sc}-\mathrm{Scd}$, and $\mathrm{Sd}-\mathrm{Sdm}$ ) reveals a clear correlation (cf. Fig. 11). Whereas the few Type I galaxies are evenly distributed with Hubble type, the frequency of Type III breaks decreases, and that of the Type II-CT increases, with later Hubble type. The Type II.o-OLR breaks appear to have a minimum for the Sc-Scd galaxies which might be related to a minimum in the overall RC3 strong bar frequency for Sc galaxies as mentioned by e.g. Lütticke (1999). We know that the Hubble type $T$ correlates with absolute magnitude $M_{\text {abs }}$ (cf. Sect. 2.1), but marking the different break types in such a plot shows that the correlation of break type is more pronounced with the Hubble type compared to $M_{\mathrm{abs}}$.

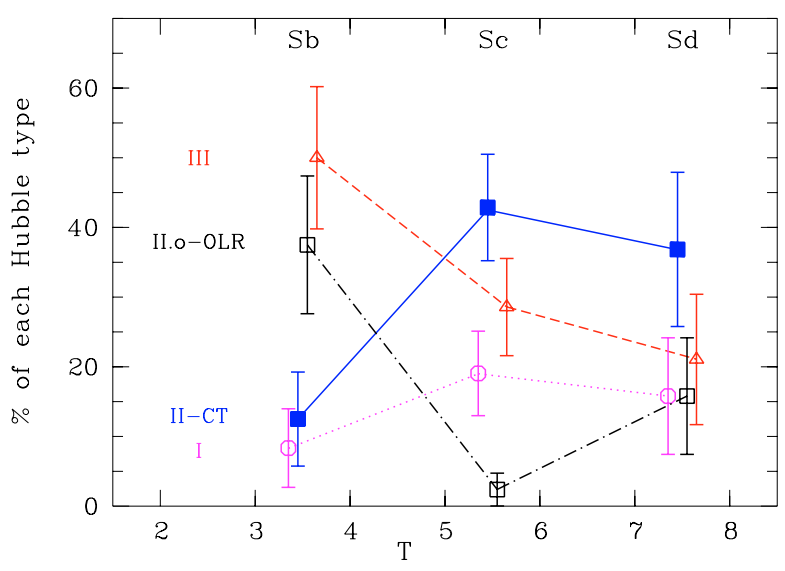

Fig. 11. Frequency of profile types Type I (open circles), Type II-CT (filled squares), Type III (open triangles), and Type II.o-OLR (open squares) in relation to the Hubble type. The galaxies are merged in three morphological bins ( $T$ between 2.5-4.4, 4.5-6.4, and 6.5-8.4). The associated points are connected with lines and slightly shifted in $T$ to be able to separate them.

For the classical truncations (Type II-CT) the position of the break at $2.5 \pm 0.6$ times the inner scalelength shows a trend with Hubble type (cf. Fig. 8) in the sense of later types being earlier truncated. However, it seems to be almost uncorrelated with total mass (either measured with $M_{\text {abs }}$ or $\mathcal{M}_{\text {dyn }}$, see Fig. 7).

\subsection{Environment}

We find for some of our Type III galaxies indication for a recent interaction (cf. Appendix A) such as close physical companions, streams, or shells. However, using the number of neighbours (as determined in Sect. 2.2), to characterise the environment, we do not find a clear correlation with the profile type. Although it looks like the Type III galaxies tend to have more neighbours within a $1 \mathrm{Mpc}$ volume (59\% have 3 or more neighbours while for the Type II-CT the fraction is only $35 \%$ ), such a correlation is far from being clear-cut. There are still many classical truncations among the galaxies having three or more (even up to nine) neighbours as well as Type III galaxies without any companion. However, we have the problem that criteria for environment are often too crude and lead even to controversial results. For example, our prototypical classical truncation, NGC 5300, has five neighbours in the SDSS database, which is clearly in a denser region according to our environment criterion. Using the CfA catalogue and applying a different criterion Varela et al. (2004) list it as being part of their truly isolated galaxy sample.

\section{Discussion}

As reported by Pohlen et al. (2004) we also do not find any galaxy with a sharp cut-off in the radial light distribution. This means that the so-called truncated galaxies are best described by a broken exponential. This seems to be now the intrinsic structure associated to the inferred sharp cut-offs proposed by van der Kruit (1979). A similar result is found, using a completely independent approach, by Ferguson et al. (2006) mapping the prototypical truncated galaxy M 33. They traced the profile down to $\mu_{\text {lim }} \sim 30 I-\mathrm{mag} / \square^{\prime \prime}$ (transformed to surface brightness) using star-counts.

In general, the shape of the transition regions we found (measured in terms of the boundary difference $b_{3}-b_{2}$ ) is no longer as 
sharp as measured by Pohlen et al. (2002). Only very few cases (e.g. NGC 5300 or NGC 7437) exhibit a very sharp transition between the inner and outer disk region. Most often the Type IICT breaks appear with a more gradual transition zone up to an almost exponential region of size $\sim 4 \mathrm{kpc}$ in between.

Since the discrimination between Type II.o-OLR and Type II-CT is solely based on bar size measurements one could argue that there are some undetected Type II.o-OLR breaks among the galaxies classified as Type II-CT. However, we carefully use either bar sizes from the literature or in most cases estimated them from the image to make sure the Type II-CT is as good as possible separated from the Type II.o-OLR breaks.

In total, without specifying the origin, most of the galaxies ( $\gtrsim 60 \%$ ) exhibit a break followed by a downbending profile almost consistent with the edge-on results by Kregel et al. (2002), although they probably would have excluded the Type II-AB galaxies from their initial sample.

We do not find truncation breaks beyond $\sim 25.2 r^{\prime}-\mathrm{mag} / \square^{\prime \prime}$ (cf. Fig. 10). We have explored whether this could be an observational bias caused by the difficulty of detecting breaks at faint levels. To do that we simulated 2D galaxies with breaks in their surface brightness distribution at different surface brightness levels. These galaxies are added to the real sky background and we measured the breaks as for real galaxies. From this we estimate our limit for detecting breaks of $\sim 26.0 r^{\prime}-\mathrm{mag} / \square^{\prime \prime}$. This limit leaves a range of $\sim 1$ mag to reach our critical surface brightness of $\mu_{\text {crit }} \sim 27.0 r^{\prime}-\mathrm{mag} / \square^{\prime \prime}$ (down to which the slope of the outer profile could be traced confidently, see Fig. 2). So we expect to detect all truncations in the region 25.2-26.0 $r^{\prime}-\mathrm{mag} / \square^{\prime \prime}$ if they do exist. Consequently, the decline observed in Fig.10 seems to be real. In addition, in terms of scalelength the largest break we find is at $R_{\mathrm{br}} / h_{\text {in }}=4.2$, but we are able to trace the profiles of our Type I galaxies down to $\sim 6-8$ radial scalelength. So we think that we do not miss any truncation breaks due to the limiting depth of the SDSS data and our Type I galaxies are indeed untruncated. This is consistent with measurements on individual galaxies being intrinsically untruncated down to $\sim 10$ scalelength (e.g. Weiner et al. 2001; Bland-Hawthorn et al. 2005). One of the key questions is now to find why some galaxies show truncations and (the minority) does not.

The strong increase for the Type III frequency towards earlier Hubble types ( $\mathrm{Sb}-\mathrm{Sbc}$ ) could be used to argue against the Type III feature being really related to the disk itself, by explaining the rise in the profile as a traditional $R^{1 / 4}$ bulge component taking over from the exponential disk in the outer parts. However, Erwin (2005) already showed that this is only true for about $\sim 1 / 3$ of the earliest types (SB0-SBb, having the highest chances of being bulge dominated). We see that $\sim 40 \%$ of our type III late-type galaxies exhibit clear signs for spiral arms in the outer disk excluding any spheroidal (pressure supported) nature of the outer structure. For another $\sim 23 \%$ of the Type III galaxies we do not find a significant decrease in ellipticity (expected if produced by a spheroidal component) while fitting free ellipses across the break. So we conclude that in most $(>63 \%)$ of our Type III galaxies the outer region is indeed related to the disk. This results fits nicely to the extended outer (starforming) disks (e.g. NGC 4625) which have been recently reported by the GALEX team (Thilker et al. 2005; Gil de Paz et al. 2005) as being significantly more frequent than thought before. Unfortunately, we do not have any galaxy classified as Type III on our SDSS images in common with those reported by the GALEX team. However, the profile shown by Swaters \& Balcells (2002) of NGC 4625 clearly suggests a Type III classification.

\section{Summary}

The main goal of our study is to provide a census of the radial disk structure of local $\left(v_{\text {vir }}<3250 \mathrm{~km} \mathrm{~s}^{-1}\right)$ late-type galaxies. Using the LEDA catalogue we selected a complete sample of 655 galaxies down to $M_{\text {abs }}=-18.4 B$-mag. 98 (85 with useful images) of them are part of the SDSS Second Data Release (DR2) which provides the imaging data used here. After careful sky subtraction we obtained the radial surface brightness profiles from fixed ellipse fits. We classified the resulting profiles (following Erwin et al. 2006) searching for clear breaks either of the downbending (steeper outer region) or upbending kind (shallower outer region) and derived scalelength and central surface brightness values for exponential fits to the individual regions.

The main conclusions are as follows:

1. We are able to reliably trace azimuthally averaged, fixed ellipse profiles of face-on to intermediate inclined galaxies down to a critical surface brightness of $\sim 27.0 r^{\prime}-\mathrm{mag} / \square^{\prime \prime}$ using SDSS imaging data. We have taken extreme care in determining the background (sky) around our objects. Our surface brightness limit value is supported by finding similar sky values with different methods. In addition, our profiles match those from available deeper photometry and we are able to recover simulated profiles added to empty SDSS sky fields down to the above surface brightness limit.

2. $90 \%$ of our galaxies could be classified into one of the following classes - Type I (no break), Type II (downbending break), and Type III (upbending break) - extending Freemans original classification. The remaining $10 \%$ of the sample could be well described being a mix from two individual classes. We introduced two main sub-classifications for the Type II class connecting the observed break with possible different physical origins: Type II-CT, classical truncations, probably associated with a global, radial starformation threshold (cf. Sect. 1), and exclusively for barred galaxies, Type II.o-OLR, OLR breaks, observed at around twice the bar radius and probably associated with the outer Lindblad resonance of the bar (see Erwin et al. 2006).

3. Surprisingly only $\lesssim 15 \%$ of all galaxies have a normal purely exponential disk down to our noise limit. A good deal more (33\% of each) have profiles with an upbending break (Type III) or a classical truncation (Type II-CT).

4. We find a correlation of break type with morphological type. Classical truncations (Type II-CT) are more frequent in later types while the fraction of upbending breaks rise towards earlier types.

5. Our Type I galaxies seem to be genuinely untruncated. The exponential profiles extend reliably down to a surface brightness of $\sim 27.0 r^{\prime}-\mathrm{mag} / \square^{\prime \prime}$ (equivalent to $\sim 6-8$ times the scalelength).

6. We do not find any galaxy with a sharp (or complete) cut-off in the radial light distribution. This means truncated galaxies are in fact best described by a broken exponential with a shallow inner and a steeper outer exponential region separated at a more or less well defined break radius.

7. For Type II-CT galaxies the break happens already at $2.5 \pm$ 0.6 times the inner scalelength at a typical surface brightness of $\mu_{\mathrm{br}}=23.5 \pm 0.8 r^{\prime}-\mathrm{mag} / \square^{\prime \prime}$. The position of the break (in $\mathrm{kpc}$ ) seems to be correlated with absolute magnitude: the more luminous the larger the inner disk of the galaxy.

8. For Type III galaxies the break happens typically further out at $4.9 \pm 0.6$ times the inner scalelength, and at a lower surface brightness of $\mu_{\mathrm{br}}=24.7 \pm 0.8 r^{\prime}-\mathrm{mag} / \square^{\prime \prime}$. For more than $60 \%$ of these galaxies we find good indication that the outer 
upbending part is a disk-like structure (e.g. by finding spiral arms). Close physical neighbours and slightly disturbed morphology suggest in several cases interaction as a possible origin.

Acknowledgements. We would like to thank Peter Erwin and John Beckman for their stimulating discussions and useful suggestions during this work and especially Peter Erwin for helping with the ellipse task and for carefully reading parts of this paper. Thanks also to Reynier Peletier for reading the paper and for suggesting to use the derivative method for objectively quantifying the break radius. We also thank Marco Barden for very stimulating discussions and we would like to thank the anonymous referee for detailed comments which helped us to improve the quality of the manuscript. Part of this work was supported by a Marie Curie Intra-European Fellowship within the 6th European Community Framework Programme. This research has made use the Lyon/Meudon Extragalactic Database (LEDA, http://leda.univ-lyon1.fr) and the NASA/IPAC Extragalactic Database (NED) which is operated by the Jet Propulsion Laboratory, California Institute of Technology, under contract with the National Aeronautics and Space Administration. This research has made use of NASA's Astrophysics Data System Bibliographic Services. Funding for the creation and distribution of the SDSS Archive has been provided by the Alfred P. Sloan Foundation, the Participating Institutions, the National Aeronautics and Space Administration, the National Science Foundation, the US Department of Energy, the Japanese Monbukagakusho, and the Max Planck Society. The SDSS Web site is http://www.sdss.org/. The SDSS is managed by the Astrophysical Research Consortium (ARC) for the Participating Institutions. The Participating Institutions are The University of Chicago, Fermilab, the Institute for Advanced Study, the Japan Participation Group, The Johns Hopkins University, the Korean Scientist Group, Los Alamos National Laboratory, the Max-Planck-Institute for Astronomy (MPIA), the Max-Planck-Institute for Astrophysics (MPA), New Mexico State University, University of Pittsburgh, University of Portsmouth, Princeton University, the United States Naval Observatory, and the University of Washington.

\section{References}

Abazajian, K., Adelman, J., Agueros, M., et al. 2004, AJ, 128, 502

Abazajian, K., Adelman, J., Agueros, M., et al. 2005, AJ, 129, 1755

Arp, H. 1966, ApJS, 14, 1

Barteldrees, A., \& Dettmar, R.-J. 1994, A\&AS, 103, 475

Barton, I. J., \& Thompson, L. A. 1997, AJ, 114, 655

Baryshev, Y. V., \& Paturel, G. 2001, A\&A, 371, 378

Binggeli, B., Sandage, A., \& Tammann, G. A. 1985, AJ, 90, 1681

Bland-Hawthorn, J., Vlajić, M., Freeman, K. C., \& Draine, B. T. 2005, ApJ, 629, 239

Bosma, A., \& Freeman, K. C. 1993, AJ, 106, 1394

Chernin, A. D. 1999, MNRAS, 308, 321

Conselice, C. J., Gallagher, J. S., Calzetti, D., Homeier, N., \& Kinney, A. 2000, AJ, 119, 79

Courteau, S. 1996, ApJS, 103, 363

Cuillandre, J.-C., Lequeux, J., Allen, R. J., Mellier, Y., \& Bertin, E. 2001, ApJ, 554,190

Debattista, V. P., Mayer, L., Carollo, C. M., et al. 2006, ApJ, 645, 209

de Grijs, R., Kregel, M., \& Wesson, K. H. 2001, MNRAS, 324, 1074

de Jong, R. S., \& van der Kruit, P. C. 1994, A\&AS, 106, 451

de Jong, R. S. 1996, A\&A, 313, 45

de Vaucouleurs, G. 1959, Handb. Phys., 53, 311

de Vaucouleurs, G., de Vaucouleurs, A., Corwin, H. G., et al. 1991, Third reference catalogue of bright galaxies (New York: Springer-Verlag)

Elmegreen, B. G., \& Hunter, D. A. 2006, ApJ, 636, 712

Erwin, P. 2004, A\&A, 415, 941

Erwin, P. 2005, MNRAS, 364, 283
Erwin, P., Beckman, J. E., \& Pohlen, M. 2005, ApJ, 626, 81

Erwin, P., Pohlen, M., \& Beckman, J. E. 2006, in preparation

Ferguson, A. M. N., Irwin, M., Chapman, S., et al. 2006, To appear in the proceedings of the conference Island Universes: Structure and Evolution of Disk Galaxies, ed. R. de Jong (Dordrecht: Springer) [arXiv: astro-ph/0601121]

Ferguson, A. M. N., Wyse, R. F. G., Gallagher, J. S., \& Hunter, D. A. 1998, ApJ, 506, L19

Florido, E., Battaner, E., Guijarro, A., Garzón, F., \& Jiménez-Vicente, J. 2001, A\&A, 378, 82

Freeman K. C. 1970, ApJ, 160, 811

Fukugita, M., Ichikawa, T., Gunn, J. E., et al. 1996, AJ, 111, 1748

Gil de Paz, A., Madore, B. F., Boissier, S., et al. 2005, ApJ, 627, L29

Giuricin, G., Marinoni, C., Ceriani, L., \& Pisani, A. 2000, ApJ, 543, 178

Graham, A. W. 2001, AJ, 121, 820

Gunn, J. E., Carr, M. A., Rockosi, C. M., et al. 1998, AJ, 116, 3040

Hunter, D. A., \& Elmegreen, B. G. 2006, ApJS, 162, 49

Jansen, R. A., Franx, M., Fabricant, D., \& Caldwell, N. 2000, ApJS, 126, 271

Jansen, R. A., \& Kannappan, S. J. 2001, Ap\&SS, 276, 1151

Jogee, S., Knapen, J. H., Laine, S., et al. 2002, ApJ, 570, L55

Karachentsev, I. D. 1972, Astrofiz. Issledov. Izvest. Spet. Astrofiz. Obse., 7, 3

Kennicutt, R. C. 1989, ApJ, 344, 685

Kregel, M., van der Kruit, P. C., \& de Grijs, R. 2002, MNRAS, 334, 646

Lütticke, R. 2001, Ph.D. Thesis, Ruhr-University Bochum, Germany

MacArthur, L. A., Courteau, S., \& Holtzman, J. A. 2003, ApJ, 582, 689

MacArthur, L. A., Courteau, S., Bell, E., \& Holtzman, J. A. 2004, ApJS, 152 , 175

Möllenhoff, C. 2004, A\&A, 415, 63

Nordgren, T. E., Chengalur, J. N., Salpeter, E. E., \& Terzian, Y. 1997, AJ, 114, 77

Patterson, F. S. 1940, Harvard Coll. Obs. Bull., 914, 9

Pérez, I. 2004, A\&A, 427, L17

Pisano, D. J., \& Wilcots, E. M. 1999, AJ, 117, 2168

Pohlen, M., Dettmar, R.-J., \& Lütticke, R. 2000a, A\&A, 357, L1

Pohlen, M. 2001, Ph.D. Thesis, Ruhr-University Bochum, Germany

Pohlen, M., Dettmar, R.-J., Lütticke, R., \& Aronica, G. 2002, A\&A, 392, 807

Pohlen, M., Beckman, J. E., Hüttemeister, S. H., et al. 2004b, in Penetrating

Bars through Masks of Cosmic Dust: The Hubble Tuning Fork Strikes a New

Note, ed. D. L. Block, I. Puerari, K. C. Freeman, R. Groess, \& E. K. Block

(Dordrecht: Springer), 713

Prada, F., Vitvitska, M., Klypin, A., et al. 2003, ApJ, 598, 260

Sandage, A., \& Bedke, J. 1994, The Carnegie atlas of galaxies, Washington, DC:

Carnegie Institution, 1994

Schaye, J. 2004, ApJ, 609, 669

Schlegel, D. J., Finkbeiner, D. P., \& Davis, M. 1998, ApJ, 500, 525

Schinnerer, E., Eckart, A., Tacconi, L. J., Genzel, R., \& Downes, D. 2000, ApJ, 533,850

Smith, J. A., Tucker, D. L., Kent, S. M., et al. 2002, AJ, 123, 2121

Swaters, R. A., \& Balcells, M. 2002, A\&A, 390, 863

Takase, B., \& Miyauchi-Isobe, N. 1984, Ann. Tokyo Astron. Obs., 19, 595

Thilker, D. A., Bianchi, L., Boissier, S., et al. 2005, ApJ, 619, L79

Trujillo, I., Asensio Ramos, A., Rubiñuo-Martin, J. A., et al. 2002, MNRAS, 333,510

Trujillo, I., \& Pohlen, M. 2005, ApJ, 630, L17

Tully, R. B. 1988, Nearby Galaxies Catalog (Cambridge: Cambrige University Press)

Tully, R. B., Verheijen, M. A. W., Pierce, M. J., Huang, J., \& Wainscoat, R. J. 1996, AJ, 112, 2471

van der Kruit, P. C. 1979 , A\&AS 38, 15

van der Kruit, P. C. 1988 , A\&A, 192, 117

van der Kruit, P. C. 1987, A\&A, 173, 59

Varela, J., Moles, M., Márquez, I., et al. 2004, A\&A, 420, 873

Weiner, B. J., Williams, T. B., van Gorkom, J. H., \& Sellwood, J. A. 2001, ApJ, 546,916

Wevers, B. M. H. R. 1984, Ph.D. Thesis, Groningen University, The Netherlands

York, D. G., Adelman, J., Anderson, J. E., et al. 2000, AJ, 120, 1579

Zaritsky, D., Smith, R., Frenk, C., \& White, S. D. M. 1993, ApJ, 405, 464 
M. Pohlen and I. Trujillo: The structure of galactic disks, Online Material $p 1$

\section{Online Material}


Table 1. Global parameters of the LEDA-SDSS DR2 subsample: (1) principal name in LEDA, (2) right ascension, and (3) declination, (4) RC3 de Vaucouleurs et al. (1991) Hubble-type, and (5) LEDA Hubble-type, (6) coded LEDA Hubble parameter $T$, (7) absolute $B$ band magnitude, corrected for galactic plus internal extinction, and k-corrected, (8) apparent diameter, defined by the isophote at the brightness of $25 B$-mag/ $\square^{\prime \prime}$, (9) heliocentric radial velocities corrected for the Local Group infall onto Virgo, (10) estimated distance according to the Hubble relation with the Hubble constant of $H_{0}=70 \mathrm{~km} \mathrm{~s}^{-1} \mathrm{Mpc}^{-1}$, (11) weighted average of the measurements maximum rotation velocity from radio (H I) and optical rotation curves $\left(\mathrm{H}_{\alpha}\right)$.

\begin{tabular}{|c|c|c|c|c|c|c|c|c|c|c|}
\hline \multirow{2}{*}{$\begin{array}{l}\text { Galaxy } \\
\text { (1) }\end{array}$} & $\begin{array}{l}\text { RA } \\
(\mathrm{J} 20\end{array}$ & $0.0)^{\text {Dec }}$ & \multirow{2}{*}{$\begin{array}{l}\mathrm{RC} 3 \\
\text { type } \\
(4)\end{array}$} & \multirow{2}{*}{$\begin{array}{l}\text { LEDA } \\
\text { type } \\
(5)\end{array}$} & \multirow{2}{*}{$\begin{array}{l}T \\
\text { (6) }\end{array}$} & \multirow{2}{*}{$\begin{array}{c}M_{\mathrm{abs}} \\
{[B-\mathrm{mag}]} \\
\text { (7) }\end{array}$} & \multirow{2}{*}{$\begin{array}{c}\text { Diam. } \\
{\left[{ }^{\prime}\right]} \\
(8)\end{array}$} & \multirow{2}{*}{$\begin{array}{c}v_{\mathrm{vir}} \\
{\left[\mathrm{km} \mathrm{s}^{-1}\right]} \\
(9)\end{array}$} & \multirow{2}{*}{$\begin{array}{l}\text { Dist. } \\
{[\mathrm{Mpc}]} \\
(10)\end{array}$} & \multirow{2}{*}{$\begin{array}{c}v_{\mathrm{rot}} \\
{\left[\mathrm{km} \mathrm{s}^{-1}\right]}\end{array}$} \\
\hline & (2) & (3) & & & & & & & & \\
\hline NGC 0428 & 011255.7 & +005854 & .SXS9.. & SBd & 8.2 & -19.39 & 3.6 & 1118 & 16.0 & 90 \\
\hline NGC 0450 & 011530.8 & -005138 & SXS6*. & $\mathrm{SBc}$ & 6.0 & -19.63 & 3.0 & 1712 & 24.5 & 118 \\
\hline PGC $006667^{\dagger}$ & 014910.2 & -100345 & .SBS7 . . & SBcd & 7.1 & -18.77 & 2.9 & 1887 & 27.0 & 129 \\
\hline NGC 0701 & 015103.8 & -094209 & .SBT5 . . & $\mathrm{SBc}$ & 5.1 & -19.74 & 2.5 & 1729 & 24.7 & 140 \\
\hline NGC 0853 & 021141.5 & -091817 & .S. . 9P? & $\mathrm{Sd}$ & 8.3 & -18.42 & 1.5 & 1405 & 20.1 & $\ldots$ \\
\hline NGC 0941 & 022827.9 & -010906 & . SXT5 . . & $\mathrm{SBc}$ & 5.4 & -19.02 & 2.5 & 1535 & 21.9 & 91 \\
\hline NGC 0988^ & 023527.4 & -092117 &. $\mathrm{SBS}^{*}$ & $\mathrm{SBc}$ & 5.8 & -20.72 & 4.5 & 1392 & 19.9 & 135 \\
\hline UGC 02081 & 023600.9 & +002512 & .SXS6. . & $\mathrm{Sc}$ & 5.8 & -18.56 & 1.8 & 2549 & 36.4 & 100 \\
\hline NGC 1042 & 024023.9 & -082558 & SXT6. . & $\mathrm{SBc}$ & 6.1 & -19.83 & 4.4 & 1264 & 18.1 & 50 \\
\hline NGC $1068^{\dagger}$ & 024240.8 & -000048 & RSAT3.. & $\mathrm{Sb}$ & 3.0 & -21.69 & 7.6 & 1068 & 15.3 & 310 \\
\hline NGC 1084 & 024559.7 & -073437 & .SAS5 . . & $\mathrm{Sc}$ & 5.1 & -20.20 & 3.2 & 1299 & 18.6 & 173 \\
\hline NGC 1087 & 024625.2 & -002955 & .SXT5 . . & $\mathrm{SBc}$ & 5.3 & -20.46 & 3.7 & 1443 & 20.6 & 125 \\
\hline NGC 1299 & 032009.4 & -061550 & SBT3?. & $\mathrm{Sb}$ & 3.2 & -19.25 & 1.2 & 2197 & 31.4 & \\
\hline NGC 2543 & 081258.0 & +361516 & .SBS3 .. & $\mathrm{SBb}$ & 3.1 & -20.66 & 2.5 & 2590 & 37.0 & 158 \\
\hline NGC 2541 & 081440.1 & +490341 & .SAS6. . & $\mathrm{SBc}$ & 6.2 & -18.50 & 5.8 & 734 & 10.5 & 97 \\
\hline UGC 04393 & 082604.3 & +455803 & SB? . . & $\mathrm{SBc}$ & 5.5 & -19.49 & 2.2 & 2290 & 32.7 & 70 \\
\hline NGC 2684 & 085454.1 & +490937 & S? ... & $\mathrm{Sd}$ & 7.8 & -19.76 & 1.0 & 3043 & 43.5 & 99 \\
\hline UGC 04684* & 085640.7 & +002230 & SAT $8 *$. & $\mathrm{Sd}$ & 7.9 & -18.86 & 1.4 & 2477 & 35.4 & 108 \\
\hline NGC 2701 & 085905.9 & +534616 & . SXT5*. & $\mathrm{SBc}$ & 5.1 & -20.36 & 2.1 & 2528 & 36.1 & 168 \\
\hline NGC $2712^{\star}$ & 085930.5 & +445450 & .SBR3*. & $\mathrm{SBb}$ & 3.2 & -20.02 & 2.9 & 1987 & 28.4 & 171 \\
\hline NGC 2776 & 091214.3 & +445717 & .SXT5 . . & $\mathrm{SBc}$ & 5.1 & -21.04 & 2.9 & 2796 & 39.9 & 118 \\
\hline NGC 2967 & 094203.3 & +002011 & . SAS5 . . & $\mathrm{Sc}$ & 4.8 & -20.26 & 2.6 & 1858 & 26.5 & 172 \\
\hline NGC 3023^ & 094952.6 & +003705 & .SXS5P* & $\mathrm{SBc}$ & 5.4 & -19.20 & 2.6 & 1852 & 26.5 & 67 \\
\hline NGC 3055 & 095517.9 & +041611 & .SXS5.. & $\mathrm{SBc}$ & 5.0 & -19.90 & 2.1 & 1816 & 25.9 & 155 \\
\hline NGC 3246 & 102641.8 & +035143 & SX. $8 \ldots$ & SBd & 8.0 & -18.91 & 2.2 & 2138 & 30.5 & 117 \\
\hline NGC 3259 & 103234.8 & +650228 & SXT4*. & $\mathrm{SBbc}$ & 4.0 & -19.84 & 2.1 & 1929 & 27.6 & 119 \\
\hline NGC 3310 & 103845.8 & +533012 & SXR4P. & SBbc & 4.0 & -20.25 & 2.8 & 1208 & 17.3 & 280 \\
\hline NGC 3359 & 104636.3 & +631328 & . SBT5 . . & $\mathrm{SBc}$ & 5.0 & -20.42 & 7.2 & 1262 & 18.0 & 139 \\
\hline PGC 032356 ${ }^{\dagger \star}$ & 104904.6 & +521957 & S? . . & $\mathrm{Sc}$ & 6.0 & -18.46 & 0.8 & 2679 & 38.3 & \\
\hline NGC 3423 & 105114.3 & +055024 & . SAS6. . & $\mathrm{Sc}$ & 6.0 & -19.54 & 3.9 & 1032 & 14.7 & 122 \\
\hline NGC 3488 & 110123.6 & +574039 & . SBS5 ${ }^{*}$ & $\mathrm{SBc}$ & 5.1 & -18.80 & 1.6 & 3226 & 46.1 & 132 \\
\hline UGC 06162^ & 110654.7 & +511212 & .5 .7$. & SBcd & 6.7 & -19.08 & 2.3 & 2426 & 34.7 & 105 \\
\hline NGC 3583 & 111411.0 & +481906 & .SBS3.. & $\mathrm{SBb}$ & 3.2 & -19.57 & 2.3 & 2347 & 33.5 & 194 \\
\hline NGC 3589 & 111513.2 & +604201 & $. S . .7 *$ & SBcd & 7.0 & -18.49 & 1.5 & 2217 & 31.7 & 75 \\
\hline UGC 06309 & 111746.4 & +512836 & SB? . . & $\mathrm{SBc}$ & 5.0 & -19.63 & 1.3 & 3097 & 44.2 & 124 \\
\hline NGC 3631 & 112102.4 & +531008 & .SAS5 . . & $\mathrm{Sc}$ & 5.1 & -20.62 & 4.9 & 1388 & 19.8 & 82 \\
\hline NGC 3642 & 112218.0 & +590427 & .SAR $4 *$ & $\mathrm{Sbc}$ & 4.0 & -20.60 & 5.5 & 1831 & 26.2 & 45 \\
\hline UGC 06518 & 113220.4 & +535417 & $\ldots \ldots$ & $\mathrm{Sbc}$ & 4.3 & -18.94 & 1.0 & 3044 & 43.5 & 84 \\
\hline NGC 3756 & 113648.3 & +541737 & .SXT4. . & SBbc & 4.3 & -19.89 & 3.8 & 1525 & 21.8 & 153 \\
\hline NGC 3888 & 114734.7 & +555800 & . SXT5.. & $\mathrm{SBc}$ & 5.3 & -20.43 & 1.8 & 2648 & 37.8 & 191 \\
\hline NGC 3893 & 114838.4 & +484234 &. $\operatorname{SXT} 5 *$ & $\mathrm{SBc}$ & 4.8 & -20.33 & 4.1 & 1193 & 17.0 & 175 \\
\hline UGC 06903 & 115536.9 & +011415 & .SBS6. . & $\mathrm{SBc}$ & 5.8 & -18.80 & 2.5 & 1916 & 27.4 & 158 \\
\hline NGC 3982 & 115628.3 & +550729 & SXR3* & $\mathrm{SBb}$ & 3.2 & -19.54 & 2.3 & 1351 & 19.3 & 193 \\
\hline NGC $3992^{\dagger}$ & 115735.9 & +532235 & .SBT4 . . & $\mathrm{SBbc}$ & 3.8 & -20.98 & 6.9 & 1286 & 18.4 & 285 \\
\hline NGC 4030 & 120023.4 & -010603 & . SAS4 . & $\mathrm{Sbc}$ & 4.1 & -20.27 & 3.9 & 1476 & 21.1 & 231 \\
\hline NGC 4041 & 120212.2 & +620814 & . SAT $4 *$ & $\mathrm{Sbc}$ & 4.3 & -19.92 & 2.7 & 1486 & 21.2 & 284 \\
\hline NGC 4102 & 120623.1 & +524239 &. SXS3\$. & $\mathrm{SBb}$ & 3.0 & -19.38 & 3.1 & 1082 & 15.5 & 169 \\
\hline NGC 4108 & 120644.0 & +670944 & PSA. $5^{*}$. & $\mathrm{Sc}$ & 5.0 & -20.13 & 1.7 & 2828 & 40.4 & 242 \\
\hline
\end{tabular}


Table 1. continued.

\begin{tabular}{|c|c|c|c|c|c|c|c|c|c|c|}
\hline \multirow{2}{*}{$\begin{array}{l}\text { Galaxy } \\
\text { (1) }\end{array}$} & $\begin{array}{l}\mathrm{RA} \\
\quad(\mathrm{J} 20)\end{array}$ & $0.0)^{\text {Dec }}$ & \multirow{2}{*}{$\begin{array}{r}\mathrm{RC} 3 \\
\text { type } \\
(4)\end{array}$} & \multirow{2}{*}{$\begin{array}{l}\text { LEDA } \\
\text { type } \\
(5)\end{array}$} & \multirow[t]{2}{*}{$T$} & \multirow{2}{*}{$\begin{array}{c}M_{\mathrm{abs}} \\
{[B-\mathrm{mag}]} \\
\text { (7) }\end{array}$} & \multirow{2}{*}{$\begin{array}{c}\text { Diam. } \\
{\left[{ }^{\prime}\right]} \\
(8)\end{array}$} & \multirow{2}{*}{$\begin{array}{c}v_{\text {vir }} \\
{\left[\mathrm{km} \mathrm{s}^{-1}\right]} \\
(9)\end{array}$} & \multirow{2}{*}{$\begin{array}{l}\text { Dist. } \\
{[\mathrm{Mpc}]} \\
(10)\end{array}$} & \multirow{2}{*}{$\begin{array}{c}v_{\mathrm{rot}} \\
{\left[\mathrm{km} \mathrm{s}^{-1}\right]}\end{array}$} \\
\hline & (2) & (3) & & & & & & & & \\
\hline NGC 4108B & 120711.3 & +671410 & .SXS7P? & SBcd & 7.0 & -18.46 & 1.3 & 2840 & 40.6 & 212 \\
\hline NGC 4116* & 120737.0 & +024129 & .SBT8. . & SBd & 7.5 & -19.32 & 3.5 & 1346 & 19.2 & 104 \\
\hline NGC 4123 & 120811.1 & +025242 & .SBR5 . . & $\mathrm{SBc}$ & 4.8 & -19.55 & 4.1 & 1364 & 19.5 & 134 \\
\hline NGC 4210 & 121515.9 & +655908 & .SBR3 . . & $\mathrm{SBb}$ & 3.0 & -19.89 & 2.0 & 3000 & 42.9 & 202 \\
\hline NGC 4273 & 121956.2 & +052036 & .SBS5 . . & $\mathrm{SBc}$ & 5.1 & -20.41 & 2.1 & 2435 & 34.8 & 187 \\
\hline NGC 4480 & 123026.8 & +041447 &. SXS5 $\ldots$ & $\mathrm{SBc}$ & 5.1 & -20.14 & 2.1 & 2494 & 35.6 & 169 \\
\hline NGC 4496A* & 123139.5 & +035622 & . SBT9. . & SBd & 8.2 & -20.25 & 3.8 & 1780 & 25.4 & 95 \\
\hline NGC 4517A & 123228.1 & +002325 & . SBT 8*. & SBd & 7.9 & -19.18 & 3.8 & 1562 & 22.3 & 73 \\
\hline UGC 07700 & 123232.8 & +635238 & .SBS8. & SBd & 7.8 & -18.50 & 1.8 & 3239 & 46.3 & 94 \\
\hline NGC 4545 & 123434.2 & +633130 & .SBS6*. & $\mathrm{SBc}$ & 5.9 & -20.49 & 2.5 & 3000 & 42.9 & 136 \\
\hline NGC 4653 & 124350.9 & -003341 & . SXT6. . & $\mathrm{SBc}$ & 6.1 & -20.37 & 3.0 & 2658 & 38.0 & 181 \\
\hline NGC 4668 & 124532.0 & -003209 &. $\mathrm{SBS} 7 *$ & SBcd & 7.1 & -18.88 & 1.5 & 1654 & 23.6 & 63 \\
\hline UGC 08041 & 125512.7 & +000700 & .SBS7 . . & SBcd & 6.8 & -18.62 & 3.2 & 1376 & 19.7 & 93 \\
\hline UGC 08084 & 125822.4 & +024733 & .SBS8 . . & SBd & 8.1 & -18.61 & 1.3 & 2824 & 40.3 & 90 \\
\hline NGC $4900^{\star}$ & 130039.2 & +023002 & .SBT5 . . & $\mathrm{SBc}$ & 5.2 & -19.01 & 2.2 & 1020 & 14.6 & 185 \\
\hline NGC 4904 & 130058.6 & -000138 & .SBS6. . & $\mathrm{SBc}$ & 5.7 & -18.69 & 2.1 & 1213 & 17.3 & 122 \\
\hline UGC 08237 & 130854.5 & +621823 & PSB. $3 *$. & $\mathrm{SBb}$ & 3.2 & -19.61 & 0.9 & 3120 & 44.6 & \\
\hline NGC 5147 & 132619.2 & +020604 & .SBS8. . & SBd & 7.9 & -18.77 & 1.8 & 1154 & 16.5 & 118 \\
\hline NGC 5218* & 133210.4 & +624604 &.$S B S 3 \$ P$ & $\mathrm{SBb}$ & 3.1 & -20.46 & 1.9 & 3154 & 45.1 & 243 \\
\hline UGC $08658^{\dagger}$ & 134039.9 & +541959 & .SXT5 . . & $\mathrm{SBc}$ & 5.1 & -19.91 & 2.6 & 2285 & 32.6 & 126 \\
\hline NGC 5300 & 134816.1 & +035703 & .SXR5 . . & $\mathrm{SBc}$ & 5.0 & -18.93 & 3. & 1246 & 17.8 & 124 \\
\hline NGC 5334 & 135254.4 & -010651 & . SBT5* & $\mathrm{SBc}$ & 5.0 & -19.22 & 3.9 & 1433 & 20.5 & 145 \\
\hline NGC 5376 & 135516.1 & +593024 &. SXR3\$. & $\mathrm{SBb}$ & 3.0 & -20.00 & 2.1 & 2293 & 32.8 & 216 \\
\hline NGC 5364^ & 135611.9 & +050055 & .SAT4P. & Sbc & 4.4 & -20.53 & 6.0 & 1322 & 18.9 & 157 \\
\hline NGC 5430 & 140045.8 & +591943 & .SBS3 . & $\mathrm{SBb}$ & 3.1 & -20.86 & 2.2 & 3238 & 46.3 & 195 \\
\hline NGC 5480 & 140621.5 & +504332 & .SAS5*. & $\mathrm{Sc}$ & 5.0 & -19.72 & 1.7 & 2119 & 30.3 & 138 \\
\hline NGC 5584 & 142223.8 & -002315 & .SXT6. . & $\mathrm{SBc}$ & 6.1 & -19.80 & 3.2 & 1702 & 24.3 & 136 \\
\hline UGC 09215^ & 142327.3 & +014332 & .SBS7 . . & SBcd & 6.6 & -18.81 & 2.2 & 1462 & 20.9 & 102 \\
\hline NGC 5624 & 142635.3 & +513503 &.$S ? \ldots$ & $\mathrm{Sc}$ & 5.0 & -18.69 & 1.1 & 2186 & 31.2 & 67 \\
\hline NGC 5660 & 142949.8 & +493722 & .SXT5. . & $\mathrm{SBc}$ & 5.1 & -20.62 & 2.8 & 2585 & 36.9 & 138 \\
\hline NGC 5667 & 143022.9 & +592811 & $. S . .6 * P$ & $\mathrm{SBc}$ & 5.8 & -19.69 & 1.7 & 2222 & 31.7 & 119 \\
\hline NGC 5668 & 143324.4 & +042702 & . SAS7 . . & Scd & 6.7 & -19.65 & 2.8 & 1672 & 23.9 & 87 \\
\hline NGC 5693 & 143611.2 & +483504 & .SBT7. . & SBcd & 6.8 & -18.64 & 1.9 & 2537 & 36.2 & 39 \\
\hline NGC 5713 & 144011.4 & -001725 & . SXT4P. & SBbc & 4.1 & -20.50 & 2.8 & 1958 & 28.0 & 135 \\
\hline NGC 5768 & 145207.9 & -023147 & . SAT5*. & $\mathrm{Sc}$ & 5.0 & -19.24 & 1. & 2018 & 28.8 & 122 \\
\hline IC 1067 & 145305.2 & +031954 &. .SBS3 $\ldots$ & $\mathrm{SBb}$ & 3.0 & -18.97 & 2.0 & 1665 & 23.8 & 158 \\
\hline NGC 5774 & 145342.6 & +033500 & . SXT7 . . & SBcd & 6.9 & -19.09 & 2.8 & 1655 & 23.6 & 91 \\
\hline NGC 5806 & 150000.4 & +015329 &. $\mathrm{SXS} 3 \ldots$ & $\mathrm{SBb}$ & 3.3 & -19.74 & 3.0 & 1440 & 20.6 & 182 \\
\hline NGC 5850 & 150707.7 & +013240 & .SBR3 . . & $\mathrm{SBb}$ & 3.0 & -21.43 & 4.3 & 2637 & 37.7 & 133 \\
\hline UGC $09741^{\dagger}$ & 150833.5 & +521746 & .S? . . . & $\mathrm{Sc}$ & 6.0 & -18.54 & 0.9 & 2735 & 39.1 & $\ldots$ \\
\hline UGC 09837 & 152351.7 & +580311 &. SXS5 $\ldots$ & Sc & 5.1 & -19.45 & 1.8 & 2938 & 42.0 & 221 \\
\hline NGC 5937 & 153046.1 & -024946 & PSXT3P. & $\mathrm{Sb}$ & 3.2 & -20.90 & 1.9 & 2870 & 41.0 & 202 \\
\hline IC 1125 & 153305.6 & -013742 & S. . 8*. & SBd & 7.7 & -20.05 & 1.7 & 2868 & 41.0 & 106 \\
\hline IC 1158 & 160134.1 & +014228 &. SXR5*. & $\mathrm{SBc}$ & 5.3 & -19.36 & 2.2 & 2018 & 28.8 & 125 \\
\hline NGC 6070 & 160958.9 & +004234 & .SAS6. . & $\mathrm{Sc}$ & 6.0 & -21.04 & 3.4 & 2085 & 29.8 & 215 \\
\hline NGC 6155 & 162608.3 & +482201 &.$S ? \ldots$ & $\mathrm{Sd}$ & 7.8 & -19.45 & 1.3 & 2684 & 38.3 & 105 \\
\hline UGC 10721 & 170825.6 & +253103 & .S. .6? & $\mathrm{Sc}$ & 5.8 & -19.68 & 1.2 & 3118 & 44.5 & 144 \\
\hline NGC 7437 & 225810.0 & +141832 & . SXT7 . . & Scd & 7.1 & -18.88 & 1.8 & 2190 & 31.3 & 175 \\
\hline NGC 7606 & 231904.8 & -082906 & .SAS3. . & $\mathrm{Sb}$ & 3.2 & -21.38 & 4.3 & 2187 & 31.2 & 291 \\
\hline UGC 12709 & 233724.0 & +002327 & .SXS9.. & $\mathrm{Sd}$ & 8.3 & -19.05 & 3.0 & 2672 & 38.2 & 70 \\
\hline
\end{tabular}

$\left(^{\star}\right)$ : Galaxy removed from further analysis (see text).

$(\dagger)$ : Galaxy with relevant alternative name: NGC $1068 \equiv$ M 77; NGC $3992 \equiv$ M 109; PGC $006667 \equiv$ UGCA 021; PGC 032356 $\equiv$ UGCA 219; UGC 08658 = Holmberg V; UGC $09741 \equiv$ NGC 5875A. 
Table 2. Background (sky) and ellipse parameters: (1) principal name in LEDA, (2+6) photometric $r^{\prime}$ and $g^{\prime}$ band zero points, (3+7) background value estimated around each galaxy, (4+8) $\sigma$ of background from the ellipse method (cf. Sect. 3.3), (5+9) limiting surface brightness due to this background, used to constrain the outer boundary of the exponential fits, (10) radius used for fixing the ellipse parameters (cf. Sect. 3.5), (11) mean position angle PA (for $r^{\prime}$ and $g^{\prime}$ band) of the fixed ellipse (as measured on the SDSS image), (12) mean ellipticity $M E$ ( $r^{\prime}$ and $g^{\prime}$ band) of the fixed ellipse.

\begin{tabular}{|c|c|c|c|c|c|c|c|c|c|c|c|}
\hline \multirow[b]{2}{*}{ Galaxy } & \multicolumn{4}{|c|}{$r^{\prime}$ band } & \multicolumn{4}{|c|}{$g^{\prime}$ band } & \multirow[b]{2}{*}{$\begin{array}{l}R_{\text {ell }} \\
{\left[{ }^{\prime \prime}\right]}\end{array}$} & \multirow[b]{2}{*}{$\begin{array}{l}\text { PA } \\
{\left[^{\circ}\right]}\end{array}$} & \multirow[b]{2}{*}{$M E$} \\
\hline & $\begin{array}{c}Z P \\
{\left[\frac{\mathrm{mag}}{\square^{\prime \prime}}\right]}\end{array}$ & $\begin{array}{r}\text { sky } \\
{[\mathrm{ADU}]}\end{array}$ & $\begin{array}{c}\sigma \\
{[\mathrm{ADU}]}\end{array}$ & $\begin{array}{c}\mu_{\lim } \\
{\left[\frac{\mathrm{mag}}{\mathrm{a}^{\prime \prime}}\right]}\end{array}$ & $\begin{array}{c}Z P \\
{\left[\frac{\mathrm{mag}}{\square^{\prime \prime}}\right]}\end{array}$ & $\begin{array}{r}\text { sky } \\
{[\mathrm{ADU}]}\end{array}$ & $\begin{array}{c}\sigma \\
{[\mathrm{ADU}]}\end{array}$ & $\begin{array}{c}\mu_{\mathrm{lim}} \\
{\left[\frac{\mathrm{mag}}{\mathrm{a}^{\prime \prime}}\right]}\end{array}$ & & & \\
\hline (1) & (2) & (3) & (4) & (5) & (6) & (7) & (8) & (9) & (10) & (11) & (12) \\
\hline NGC 0450 & 26.267 & 165.11 & 0.14 & 27.2 & 26.686 & 99.19 & 0.09 & 28.1 & 84 & -8.8 & 0.36 \\
\hline PGC 006667 & 26.213 & 175.59 & 0.06 & 28.1 & 26.451 & 74.16 & 0.12 & 27.6 & 67 & 35.8 & 0.19 \\
\hline 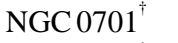 & 26.136 & 157.72 & 0.08 & 27.7 & 26.343 & 69.07 & 0.09 & 27.8 & 74 & -41.9 & 0.49 \\
\hline NGC $0853^{\dagger}$ & 26.293 & 144.89 & 0.11 & 27.5 & 26.542 & 75.84 & 0.07 & 28.2 & 59 & -11.7 & 0.36 \\
\hline NGC 0941 & 26.114 & 99.42 & 0.02 & 29.2 & 26.507 & 68.58 & 0.11 & 27.7 & 69 & 78.6 & 0.30 \\
\hline UGC 02081 & 26.106 & 99.91 & 0.03 & 28.7 & 26.282 & 56.34 & 0.08 & 27.8 & 51 & -18.8 & 0.42 \\
\hline NGC 1042 & 26.266 & 175.77 & 0.09 & 27.7 & 26.508 & 75.11 & 0.07 & 28.2 & 136 & -69.0 & 0.20 \\
\hline NGC 1068 & 26.248 & 186.31 & 0.12 & 27.4 & 26.551 & 94.08 & 0.09 & 28.0 & 312 & 9.8 & 0.09 \\
\hline NGC 1084 & 26.259 & 170.55 & 0.18 & 26.9 & 26.416 & 70.41 & $*$ & $*$ & 80 & -46.9 & 0.39 \\
\hline NGC $1087^{\dagger}$ & 26.246 & 175.97 & 0.09 & 27.7 & 26.533 & 92.17 & 0.08 & 28.1 & 114 & -88.4 & 0.39 \\
\hline NGC 1299 & 26.275 & 190.56 & 0.13 & 27.3 & 26.480 & 82.11 & 0.14 & 27.4 & 43 & -35.0 & 0.45 \\
\hline NGC 2543 & 26.271 & 129.29 & 0.17 & 27.0 & 26.563 & 79.22 & 0.09 & 28.0 & 108 & -3.1 & 0.50 \\
\hline NGC $2541^{\dagger}$ & 26.259 & 181.06 & 0.10 & 27.6 & 26.532 & 91.73 & 0.04 & 28.8 & 135 & -53.5 & 0.52 \\
\hline UGC $04393^{\dagger}$ & 26.345 & 116.47 & 0.08 & 27.9 & 26.564 & 66.68 & 0.07 & 28.3 & 61 & 11.9 & 0.45 \\
\hline NGC 2684 & 26.314 & 122.01 & 0.08 & 27.9 & 26.591 & 70.12 & 0.19 & 27.2 & 35 & 8.7 & 0.18 \\
\hline NGC $2701^{\dagger}$ & 26.360 & 162.44 & 0.09 & 27.8 & 26.653 & 86.65 & 0.08 & 28.2 & 60 & -19.3 & 0.32 \\
\hline NGC 2776 & 26.291 & 156.28 & 0.05 & 28.4 & 26.508 & 67.37 & 0.05 & 28.6 & 83 & 30.8 & 0.05 \\
\hline NGC 2967 & 26.278 & 150.33 & 0.06 & 28.1 & 26.605 & 106.62 & 0.12 & 27.7 & 77 & -70.7 & 0.07 \\
\hline NGC 3055 & 26.255 & 117.73 & 0.11 & 27.5 & 26.566 & 73.52 & 0.11 & 27.8 & 65 & -24.1 & 0.42 \\
\hline NGC $3246^{\dagger}$ & 26.281 & 125.07 & 0.11 & 27.5 & 26.539 & 72.34 & 0.09 & 28.0 & 65 & 8.0 & 0.48 \\
\hline NGC 3259 & 26.260 & 158.22 & 0.14 & 27.2 & 26.530 & 70.55 & 0.07 & 28.2 & 53 & -47.0 & 0.43 \\
\hline NGC 3310 & 26.229 & 134.53 & 0.07 & 27.9 & 26.527 & 70.73 & 0.24 & 26.9 & 83 & -50.2 & 0.05 \\
\hline NGC 3359 & 26.274 & 150.77 & 0.08 & 27.8 & 26.623 & 84.83 & 0.10 & 27.9 & 236 & -80.9 & 0.48 \\
\hline NGC 3423 & 26.286 & 103.65 & 0.07 & 28.0 & 26.720 & 75.84 & 0.10 & 28.0 & 112 & -53.5 & 0.19 \\
\hline NGC 3488 & 26.266 & 119.86 & 0.10 & 27.6 & 26.548 & 70.64 & 0.08 & 28.1 & 52 & -75.8 & 0.34 \\
\hline NGC 3583 & 26.270 & 104.52 & 0.11 & 27.5 & 26.550 & 59.18 & 0.08 & 28.1 & 81 & 46.1 & 0.32 \\
\hline NGC $3589^{\dagger}$ & 26.312 & 139.12 & 0.10 & 27.6 & 26.567 & 60.49 & 0.03 & 29.2 & 46 & -21.9 & 0.50 \\
\hline UGC 06309 & 26.241 & 109.30 & 0.07 & 27.9 & 26.569 & 58.96 & 0.05 & 28.6 & 50 & 45.8 & 0.31 \\
\hline NGC 3631 & 26.261 & 140.70 & 0.07 & 28.0 & 26.661 & 77.36 & 0.06 & 28.5 & 129 & 32.1 & 0.16 \\
\hline NGC 3642 & 26.266 & 94.01 & 0.05 & 28.3 & 26.569 & 54.00 & 0.04 & 28.9 & 77 & 5.1 & 0.05 \\
\hline UGC 06518 & 26.263 & 137.25 & 0.13 & 27.3 & 26.514 & 65.27 & 0.08 & 28.1 & 31 & -59.0 & 0.39 \\
\hline NGC 3756 & 26.205 & 135.84 & 0.17 & 26.9 & 26.507 & 67.02 & 0.14 & 27.4 & 118 & -82.2 & 0.50 \\
\hline NGC 3888 & 26.223 & 133.12 & 0.07 & 27.9 & 26.512 & 65.09 & 0.05 & 28.6 & 52 & 30.9 & 0.26 \\
\hline NGC 3893 & 26.279 & 107.16 & 0.09 & 27.7 & 26.691 & 66.61 & 0.10 & 28.0 & 133 & 83.6 & 0.41 \\
\hline UGC 06903 & 26.264 & 136.09 & 0.04 & 28.6 & 26.571 & 76.94 & 0.08 & 28.1 & 69 & 48.1 & 0.12 \\
\hline NGC 3982 & 26.204 & 133.59 & 0.11 & 27.4 & 26.540 & 68.43 & 0.04 & 28.8 & 58 & -87.4 & 0.11 \\
\hline NGC 3992 & 26.235 & 113.06 & 0.09 & 27.7 & 26.517 & 59.44 & 0.18 & 27.2 & 264 & -15.4 & 0.42 \\
\hline NGC 4030 & 26.261 & 137.95 & 0.06 & 28.1 & 26.707 & 96.55 & 0.09 & 28.1 & 124 & -59.6 & 0.20 \\
\hline NGC 4041 & 26.353 & 152.10 & 0.05 & 28.4 & 26.596 & 62.19 & 0.07 & 28.3 & 84 & -8.5 & 0.05 \\
\hline NGC 4102 & 26.267 & 123.57 & 0.06 & 28.1 & 26.594 & 68.12 & 0.08 & 28.1 & 94 & -47.9 & 0.43 \\
\hline NGC $4108^{\dagger}$ & 26.317 & 174.08 & 0.11 & 27.5 & 26.603 & 92.76 & 0.06 & 28.5 & 55 & 13.2 & 0.22 \\
\hline NGC $4108 B^{\dagger}$ & 26.317 & 173.86 & 0.06 & 28.2 & 26.603 & 92.71 & 0.03 & 29.2 & 37 & 39.8 & 0.23 \\
\hline NGC 4123 & 26.195 & 161.48 & 0.09 & 27.6 & 26.524 & 90.50 & 0.11 & 27.7 & 111 & 36.1 & 0.33 \\
\hline NGC 4210 & 26.290 & 119.97 & 0.04 & 28.6 & 26.607 & 62.28 & 0.05 & 28.7 & 55 & 9.3 & 0.24 \\
\hline NGC $4273^{\dagger}$ & 26.252 & 130.84 & 0.10 & 27.6 & 26.566 & 73.89 & 0.11 & 27.8 & 72 & -83.3 & 0.39 \\
\hline NGC 4480 & 26.228 & 143.92 & 0.08 & 27.8 & 26.489 & 77.53 & 0.08 & 28.0 & 65 & 87.2 & 0.50 \\
\hline
\end{tabular}


Table 2. continued.

\begin{tabular}{|c|c|c|c|c|c|c|c|c|c|c|c|}
\hline \multirow[b]{2}{*}{ Galaxy } & \multicolumn{4}{|c|}{$r^{\prime}$ band } & \multicolumn{4}{|c|}{$g^{\prime}$ band } & \multirow[b]{2}{*}{$\begin{array}{l}R_{\text {ell }} \\
{\left[{ }^{\prime \prime}\right]}\end{array}$} & \multirow[b]{2}{*}{$\begin{array}{l}P A \\
{\left[{ }^{\circ}\right]}\end{array}$} & \multirow[b]{2}{*}{$M E$} \\
\hline & $\begin{array}{c}Z P \\
{\left[\frac{\mathrm{mag}}{\mathrm{a}^{\prime \prime}}\right]}\end{array}$ & $\begin{array}{r}\text { sky } \\
{[\mathrm{ADU}]}\end{array}$ & $\begin{array}{c}\sigma \\
{[\mathrm{ADU}]}\end{array}$ & $\begin{array}{c}\mu_{\mathrm{lim}} \\
{\left[\frac{\mathrm{mag}}{\square^{\prime \prime}}\right]}\end{array}$ & $\begin{array}{c}Z P \\
{\left[\frac{\mathrm{mag}}{\square^{\prime \prime}}\right]}\end{array}$ & $\begin{array}{r}\text { sky } \\
{[\mathrm{ADU}]}\end{array}$ & $\begin{array}{c}\sigma \\
{[\mathrm{ADU}]}\end{array}$ & $\begin{array}{c}\mu_{\mathrm{lim}} \\
{\left[\frac{\mathrm{mag}}{\mathrm{a}^{\prime \prime}}\right]}\end{array}$ & & & \\
\hline (1) & (2) & (3) & (4) & (5) & (6) & (7) & (8) & (9) & (10) & (11) & (12) \\
\hline NGC 4517A & 26.278 & 136.44 & 0.07 & 28.0 & 26.605 & 78.57 & 0.08 & 28.2 & 113 & -61.4 & 0.43 \\
\hline UGC $07700^{\dagger}$ & 26.346 & 124.40 & 0.02 & 29.4 & 26.766 & 71.96 & 0.13 & 27.8 & 66 & -23.6 & 0.24 \\
\hline NGC 4545 & 26.225 & 115.06 & 0.04 & 28.5 & 26.541 & 54.71 & 0.07 & 28.2 & 66 & -87.9 & 0.42 \\
\hline NGC 4653 & 26.292 & 135.45 & 0.05 & 28.4 & 26.584 & 75.43 & 0.08 & 28.1 & 79 & 78.5 & 0.17 \\
\hline NGC 4668 & 26.292 & 135.17 & 0.07 & 28.0 & 26.584 & 75.49 & 0.09 & 28.0 & 53 & -85.5 & 0.48 \\
\hline UGC 08041 & 26.253 & 127.60 & 0.11 & 27.5 & 26.566 & 78.23 & 0.14 & 27.5 & 101 & 70.0 & 0.48 \\
\hline UGC $08084^{\dagger}$ & 26.195 & 157.33 & 0.05 & 28.3 & 26.524 & 86.02 & 0.01 & 30.3 & 43 & -30.9 & 0.18 \\
\hline NGC 4904 & 26.322 & 142.49 & 0.08 & 27.9 & 26.588 & 75.36 & 0.06 & 28.4 & 70 & -63.4 & 0.29 \\
\hline UGC 08237 & 26.228 & 103.85 & 0.11 & 27.4 & 26.574 & 56.11 & 0.03 & 29.2 & 35 & 30.7 & 0.22 \\
\hline NGC 5147 & 26.242 & 179.24 & 0.04 & 28.5 & 26.494 & 92.26 & 0.06 & 28.4 & 60 & 28.8 & 0.21 \\
\hline UGC 08658 & 26.174 & 122.81 & 0.13 & 27.2 & 26.465 & 66.52 & 0.07 & 28.2 & 65 & 3.2 & 0.39 \\
\hline NGC 5300 & 26.235 & 146.52 & 0.09 & 27.7 & 26.495 & 81.87 & 0.09 & 27.9 & 100 & 58.5 & 0.33 \\
\hline NGC 5334 & 26.261 & 125.54 & 0.07 & 28.0 & 26.707 & 86.37 & 0.05 & 28.8 & 107 & -76.0 & 0.23 \\
\hline NGC 5376 & 26.237 & 108.94 & 0.11 & 27.4 & 26.528 & 56.56 & 0.03 & 29.1 & 63 & -49.8 & 0.39 \\
\hline NGC 5430 & 26.237 & 108.70 & 0.07 & 27.9 & 26.528 & 55.89 & 0.05 & 28.6 & 69 & 70.5 & 0.35 \\
\hline NGC 5480 & 26.226 & 149.31 & 0.08 & 27.8 & 26.670 & 69.86 & 0.08 & 28.2 & 60 & -73.3 & 0.15 \\
\hline NGC 5584 & 26.312 & 135.42 & 0.08 & 27.9 & 26.567 & 74.06 & 0.07 & 28.3 & 106 & 67.9 & 0.26 \\
\hline NGC $5624^{\dagger}$ & 26.163 & 147.69 & 0.09 & 27.6 & 26.487 & 63.15 & 0.09 & 27.9 & 38 & 82.8 & 0.34 \\
\hline NGC 5660 & 26.199 & 123.03 & 0.06 & 28.1 & 26.488 & 64.66 & 0.08 & 28.0 & 69 & 24.3 & 0.05 \\
\hline NGC 5667 & 26.216 & 99.47 & 0.09 & 27.6 & 26.566 & 55.67 & 0.04 & 28.9 & 55 & 51.3 & 0.47 \\
\hline NGC 5668 & 26.257 & 117.18 & 0.05 & 28.3 & 26.578 & 70.51 & 0.09 & 28.0 & 95 & 43.2 & 0.15 \\
\hline NGC $5693^{\dagger}$ & 26.225 & 151.52 & 0.08 & 27.8 & 26.669 & 74.68 & 0.09 & 28.1 & 61 & 13.2 & 0.05 \\
\hline NGC $5713^{\dagger}$ & 26.312 & 134.21 & 0.04 & 28.6 & 26.567 & 73.88 & 0.06 & 28.4 & 88 & -81.6 & 0.13 \\
\hline NGC $5768^{\dagger}$ & 26.212 & 128.52 & 0.07 & 27.9 & 26.493 & 76.07 & 0.03 & 29.1 & 70 & 24.1 & 0.11 \\
\hline IC 1067 & 26.261 & 123.11 & 0.09 & 27.7 & 26.537 & 69.83 & 0.17 & 27.3 & 60 & 25.8 & 0.26 \\
\hline NGC $5774^{\dagger}$ & 26.254 & 173.84 & 0.06 & 28.1 & 26.522 & 85.40 & 0.06 & 28.4 & 87 & 41.8 & 0.22 \\
\hline NGC $5806^{\dagger}$ & 26.246 & 179.83 & 0.09 & 27.7 & 26.518 & 82.76 & 0.06 & 28.4 & 79 & 73.7 & 0.46 \\
\hline NGC 5850 & 26.241 & 195.14 & 0.09 & 27.7 & 26.492 & 94.97 & 0.06 & 28.4 & 141 & 74.9 & 0.22 \\
\hline UGC 09741 & 26.192 & 126.43 & 0.04 & 28.5 & 26.548 & 56.25 & 0.05 & 28.6 & 45 & 42.2 & 0.11 \\
\hline UGC 09837 & 26.246 & 115.43 & 0.08 & 27.8 & 26.731 & 62.31 & 0.07 & 28.4 & 53 & 20.9 & 0.05 \\
\hline NGC 5937 & 26.232 & 121.74 & 0.07 & 27.9 & 26.650 & 84.21 & 0.12 & 27.8 & 61 & -69.1 & 0.44 \\
\hline IC 1125 & 26.189 & 113.30 & 0.12 & 27.3 & 26.525 & 74.76 & 0.09 & 27.9 & 46 & 56.0 & 0.44 \\
\hline IC 1158 & 26.233 & 164.49 & 0.10 & 27.5 & 26.689 & 112.46 & 0.12 & 27.8 & 69 & 39.1 & 0.44 \\
\hline NGC 6070 & 26.296 & 149.32 & 0.15 & 27.2 & 26.519 & 63.01 & 0.13 & 27.5 & 114 & -30.8 & 0.57 \\
\hline NGC $6155^{\dagger}$ & 26.304 & 125.81 & 0.07 & 28.0 & 26.584 & 63.54 & 0.11 & 27.8 & 47 & -7.7 & 0.28 \\
\hline UGC 10721 & 26.198 & 118.98 & 0.10 & 27.5 & 26.524 & 55.93 & 0.05 & 28.6 & 38 & -31.0 & 0.33 \\
\hline NGC 7437 & 26.191 & 153.77 & 0.12 & 27.3 & 26.495 & 87.08 & 0.09 & 27.9 & 57 & -65.7 & 0.10 \\
\hline NGC 7606 & 26.278 & 225.46 & 0.12 & 27.4 & 26.576 & 89.15 & 0.17 & 27.3 & 146 & 51.7 & 0.62 \\
\hline UGC $12709^{\dagger}$ & 26.236 & 154.79 & 0.11 & 27.4 & 26.565 & 80.44 & 0.07 & 28.3 & 71 & 50.4 & 0.39 \\
\hline
\end{tabular}

$\dagger$ : The centers for these galaxies are not obtained by a Gaussian fit to the central region but by a free ellipse fit to determine the best center representative for the outer disk. 
Table 3. Results: disk type and exponential disk parameters. (1) Principal name in LEDA, (2) profile classification (cf. Sect. 4.1), (3) fitting boundaries for the inner and outer exponential disk region, (4) break radius in units of arcsec and kpc, (5) inner scalelength in units of arcsec and kpc (6) inner scalelength in relation to the outer scalelength, (7) the central surface brightness of the inner/outer disk, (8) the surface brightness at the break radius (estimated at the crossing point of the two exponential fits), (9) galactic extinction according to Schlegel et al. (1998). For each galaxy two rows of values are given. The results obtained for the $r^{\prime}$ band in the upper row, for the $g^{\prime}$ band in the lower row. For galaxies with mixed classification both disk fits are shown.

\begin{tabular}{|c|c|c|c|c|c|c|c|c|c|c|c|c|c|c|}
\hline \multirow{3}{*}{$\begin{array}{l}\text { Galaxy } \\
\text { (1) }\end{array}$} & \multirow{3}{*}{$\begin{array}{c}\text { Break } \\
\text { type } \\
\text { (2) }\end{array}$} & \multirow{3}{*}{$\begin{array}{c}b_{1} \\
{\left[{ }^{\prime \prime}\right]}\end{array}$} & \multirow{2}{*}{$\begin{array}{c}b_{2} \\
{\left[{ }^{\prime \prime}\right]}\end{array}$} & \multirow{2}{*}{$\begin{array}{c}b_{3} \\
{\left[{ }^{\prime \prime}\right]}\end{array}$} & \multirow{3}{*}{$\begin{array}{c}b_{4} \\
{\left[{ }^{\prime \prime}\right]}\end{array}$} & \multicolumn{2}{|c|}{$\overline{\overline{R_{\mathrm{br}}}}$} & \multicolumn{2}{|c|}{$\overline{h_{\text {in }}}$} & \multirow{3}{*}{$\begin{array}{c}h_{\text {in }} \\
\left.h_{\text {out }}\right] \\
(6)\end{array}$} & \multirow{2}{*}{$\begin{array}{c}\mu_{0, \text { in }} \\
{\left[\frac{\mathrm{mag}}{\mathrm{a}^{\prime \prime}}\right]}\end{array}$} & \multirow{3}{*}{$\begin{array}{l}\mu_{0, \text { out }} \\
{\left[\frac{\mathrm{mag}}{\mathrm{D}^{\prime \prime}}\right]}\end{array}$} & \multirow{3}{*}{$\begin{array}{c}\mu_{\mathrm{br}} \\
{\left[\frac{\mathrm{mag}}{\square^{\prime \prime}}\right]} \\
(8)\end{array}$} & \multirow{3}{*}{$\begin{array}{c}A_{r^{\prime} / g^{\prime}} \\
{[\mathrm{mag}]} \\
(9)\end{array}$} \\
\hline & & & & & & {$\left[{ }^{\prime \prime}\right]$} & [kpc] & {$\left[{ }^{\prime \prime}\right]$} & {$[\mathrm{kpc}]$} & & & & & \\
\hline & & & \multicolumn{2}{|c|}{ (3) } & & \multicolumn{2}{|c|}{ (4) } & \multicolumn{2}{|c|}{ (5) } & & (7) & & & \\
\hline NGC 0450 & II.o-CT & $\begin{array}{l}16 \\
16\end{array}$ & $\begin{array}{l}79 \\
78\end{array}$ & $\begin{array}{l}88 \\
89\end{array}$ & $\begin{array}{l}120 \\
128\end{array}$ & $\begin{array}{l}80 \\
79\end{array}$ & $\begin{array}{l}9.5 \\
9.4\end{array}$ & $\begin{array}{l}31.3 \\
34.5\end{array}$ & $\begin{array}{l}3.7 \\
4.1\end{array}$ & $\begin{array}{l}2.7 \\
2.9\end{array}$ & $\begin{array}{l}21.0 \\
21.5\end{array}$ & $\begin{array}{l}16.0 \\
16.3\end{array}$ & $\begin{array}{l}24.0 \\
24.2\end{array}$ & $\begin{array}{l}0.11 \\
0.15\end{array}$ \\
\hline PGC 006667 & II-AB & $\begin{array}{l}10 \\
10\end{array}$ & $\begin{array}{l}70 \\
72\end{array}$ & $\begin{array}{l}77 \\
78\end{array}$ & $\begin{array}{r}108 \\
96\end{array}$ & $\begin{array}{l}74 \\
73\end{array}$ & $\begin{array}{l}9.7 \\
9.5\end{array}$ & $\begin{array}{l}22.6 \\
22.7\end{array}$ & $\begin{array}{l}2.9 \\
3.0\end{array}$ & $\begin{array}{l}1.9 \\
1.8\end{array}$ & $\begin{array}{l}21.1 \\
21.5\end{array}$ & $\begin{array}{l}18.0 \\
18.7\end{array}$ & $\begin{array}{l}24.7 \\
25.1\end{array}$ & $\begin{array}{l}0.14 \\
0.19\end{array}$ \\
\hline NGC 0701 & II-i & $\begin{array}{l}19 \\
19\end{array}$ & $\begin{array}{l}129 \\
115\end{array}$ & $*$ & * & $*$ & $*$ & $\begin{array}{l}15.4 \\
16.2\end{array}$ & $\begin{array}{l}1.8 \\
1.9\end{array}$ & * & $\begin{array}{l}19.0 \\
19.8\end{array}$ & $\begin{array}{l}0.0 \\
0.0\end{array}$ & $\begin{array}{l}19.0 \\
19.8\end{array}$ & $\begin{array}{l}0.07 \\
0.10\end{array}$ \\
\hline NGC 0853 & III & $\begin{array}{l}0 \\
0\end{array}$ & $\begin{array}{l}43 \\
42\end{array}$ & $\begin{array}{l}48 \\
46\end{array}$ & $\begin{array}{l}100 \\
100\end{array}$ & $\begin{array}{l}45 \\
44\end{array}$ & $\begin{array}{l}4.4 \\
4.3\end{array}$ & $\begin{array}{l}11.0 \\
10.3\end{array}$ & $\begin{array}{l}1.1 \\
1.0\end{array}$ & $\begin{array}{l}0.6 \\
0.6\end{array}$ & $\begin{array}{l}19.3 \\
19.6\end{array}$ & $\begin{array}{l}20.9 \\
21.2\end{array}$ & $\begin{array}{l}23.3 \\
23.7\end{array}$ & $\begin{array}{l}0.07 \\
0.10\end{array}$ \\
\hline NGC 0941 & II-CT & $\begin{array}{l}5 \\
5\end{array}$ & $\begin{array}{l}77 \\
76\end{array}$ & $\begin{array}{l}84 \\
80\end{array}$ & $\begin{array}{l}120 \\
107\end{array}$ & $\begin{array}{l}81 \\
77\end{array}$ & $\begin{array}{l}8.6 \\
8.2\end{array}$ & $\begin{array}{l}19.1 \\
19.5\end{array}$ & $\begin{array}{l}2.0 \\
2.1\end{array}$ & $\begin{array}{l}1.4 \\
1.6\end{array}$ & $\begin{array}{l}20.4 \\
20.8\end{array}$ & $\begin{array}{l}18.5 \\
18.0\end{array}$ & $\begin{array}{l}25.0 \\
25.3\end{array}$ & $\begin{array}{l}0.10 \\
0.13\end{array}$ \\
\hline UGC 02081 & II-CT & $\begin{array}{l}11 \\
11\end{array}$ & $\begin{array}{l}48 \\
45\end{array}$ & $\begin{array}{l}69 \\
74\end{array}$ & $\begin{array}{l}85 \\
85\end{array}$ & $\begin{array}{l}53 \\
46\end{array}$ & $\begin{array}{l}9.4 \\
8.1\end{array}$ & $\begin{array}{l}21.0 \\
22.3\end{array}$ & $\begin{array}{l}3.7 \\
3.9\end{array}$ & $\begin{array}{l}2.6 \\
2.8\end{array}$ & $\begin{array}{l}21.6 \\
22.2\end{array}$ & $\begin{array}{l}16.1 \\
15.9\end{array}$ & $\begin{array}{l}25.2 \\
25.6\end{array}$ & $\begin{array}{l}0.07 \\
0.10\end{array}$ \\
\hline NGC 1042 & II-AB & $\begin{array}{l}39 \\
39\end{array}$ & $\begin{array}{l}122 \\
101\end{array}$ & $\begin{array}{l}122 \\
119\end{array}$ & $\begin{array}{l}219 \\
212\end{array}$ & $\begin{array}{l}122 \\
119\end{array}$ & $\begin{array}{l}10.7 \\
10.4\end{array}$ & $\begin{array}{l}48.0 \\
63.9\end{array}$ & $\begin{array}{l}4.2 \\
5.6\end{array}$ & $\begin{array}{l}1.4 \\
2.2\end{array}$ & $\begin{array}{l}20.9 \\
21.7\end{array}$ & $\begin{array}{l}19.9 \\
19.7\end{array}$ & $\begin{array}{l}23.0 \\
23.4\end{array}$ & $\begin{array}{l}0.08 \\
0.11\end{array}$ \\
\hline NGC 1068 & II.o-OLR & $\begin{array}{l}105 \\
105\end{array}$ & $\begin{array}{l}184 \\
183\end{array}$ & $\begin{array}{l}188 \\
187\end{array}$ & $\begin{array}{l}362 \\
384\end{array}$ & $\begin{array}{l}188 \\
189\end{array}$ & $\begin{array}{l}13.9 \\
14.0\end{array}$ & $\begin{array}{l}118.0 \\
128.2\end{array}$ & $\begin{array}{l}8.7 \\
9.5\end{array}$ & $\begin{array}{l}2.0 \\
2.2\end{array}$ & $\begin{array}{l}21.6 \\
22.4\end{array}$ & $\begin{array}{l}19.9 \\
20.4\end{array}$ & $\begin{array}{l}23.4 \\
24.0\end{array}$ & $\begin{array}{l}0.09 \\
0.13\end{array}$ \\
\hline NGC 1084 & III & $\begin{array}{l}27 \\
27\end{array}$ & $\begin{array}{l}91 \\
91\end{array}$ & $\begin{array}{r}97 \\
100\end{array}$ & $\begin{array}{l}179 \\
194\end{array}$ & $\begin{array}{l}95 \\
96\end{array}$ & $\begin{array}{l}8.5 \\
8.6\end{array}$ & $\begin{array}{l}18.5 \\
17.9\end{array}$ & $\begin{array}{l}1.7 \\
1.6\end{array}$ & $\begin{array}{l}0.4 \\
0.4\end{array}$ & $\begin{array}{l}18.3 \\
18.7\end{array}$ & $\begin{array}{l}21.4 \\
21.7\end{array}$ & $\begin{array}{l}23.2 \\
23.7\end{array}$ & $\begin{array}{l}0.08 \\
0.11\end{array}$ \\
\hline NGC 1087 & III & $\begin{array}{l}49 \\
49\end{array}$ & $\begin{array}{l}139 \\
137\end{array}$ & $\begin{array}{l}142 \\
142\end{array}$ & $\begin{array}{l}238 \\
231\end{array}$ & $\begin{array}{l}140 \\
139\end{array}$ & $\begin{array}{l}14.0 \\
13.9\end{array}$ & $\begin{array}{l}24.5 \\
23.6\end{array}$ & $\begin{array}{l}2.4 \\
2.4\end{array}$ & $\begin{array}{l}0.6 \\
0.6\end{array}$ & $\begin{array}{l}19.0 \\
19.4\end{array}$ & $\begin{array}{l}21.4 \\
22.0\end{array}$ & $\begin{array}{l}24.7 \\
25.4\end{array}$ & $\begin{array}{l}0.10 \\
0.13\end{array}$ \\
\hline NGC 1299 & III & $\begin{array}{l}22 \\
22\end{array}$ & $\begin{array}{l}45 \\
47\end{array}$ & $\begin{array}{l}46 \\
52\end{array}$ & $\begin{array}{l}75 \\
77\end{array}$ & $\begin{array}{l}46 \\
49\end{array}$ & $\begin{array}{l}7.0 \\
7.5\end{array}$ & $\begin{array}{l}8.7 \\
8.6\end{array}$ & $\begin{array}{l}1.3 \\
1.3\end{array}$ & $\begin{array}{l}0.7 \\
0.5\end{array}$ & $\begin{array}{l}18.9 \\
19.4\end{array}$ & $\begin{array}{l}20.5 \\
22.5\end{array}$ & $\begin{array}{l}24.5 \\
25.4\end{array}$ & $\begin{array}{l}0.13 \\
0.18\end{array}$ \\
\hline NGC 2543 & II-i & $\begin{array}{l}34 \\
34\end{array}$ & $\begin{array}{l}108 \\
108\end{array}$ & * & * & $\begin{array}{l}74 \\
75\end{array}$ & $\begin{array}{l}13.3 \\
13.5\end{array}$ & $\begin{array}{l}15.7 \\
14.3\end{array}$ & $\begin{array}{l}2.8 \\
2.6\end{array}$ & * & $\begin{array}{l}19.1 \\
19.3\end{array}$ & $\begin{array}{l}0.0 \\
0.0\end{array}$ & $\begin{array}{l}19.1 \\
19.3\end{array}$ & $\begin{array}{l}0.19 \\
0.27\end{array}$ \\
\hline NGC 2541 & II-CT & $\begin{array}{l}48 \\
48\end{array}$ & $\begin{array}{l}141 \\
143\end{array}$ & $\begin{array}{l}143 \\
147\end{array}$ & $\begin{array}{l}216 \\
223\end{array}$ & $\begin{array}{l}142 \\
145\end{array}$ & $\begin{array}{l}7.2 \\
7.4\end{array}$ & $\begin{array}{l}51.2 \\
55.2\end{array}$ & $\begin{array}{l}2.6 \\
2.8\end{array}$ & $\begin{array}{l}1.6 \\
2.1\end{array}$ & $\begin{array}{l}21.3 \\
21.9\end{array}$ & $\begin{array}{l}19.3 \\
18.6\end{array}$ & $\begin{array}{l}24.5 \\
25.0\end{array}$ & $\begin{array}{l}0.14 \\
0.19\end{array}$ \\
\hline UGC 04393 & I & $\begin{array}{l}30 \\
30\end{array}$ & $\begin{array}{l}125 \\
114\end{array}$ & * & * & * & * & $\begin{array}{l}22.4 \\
21.7\end{array}$ & $\begin{array}{l}3.6 \\
3.4\end{array}$ & * & $\begin{array}{l}21.6 \\
21.9\end{array}$ & $\begin{array}{l}0.0 \\
0.0\end{array}$ & $\begin{array}{l}21.6 \\
21.9\end{array}$ & $\begin{array}{l}0.12 \\
0.16\end{array}$ \\
\hline NGC 2684 & II-CT & $\begin{array}{l}4 \\
4\end{array}$ & $\begin{array}{l}23 \\
23\end{array}$ & $\begin{array}{l}25 \\
25\end{array}$ & $\begin{array}{l}42 \\
42\end{array}$ & $\begin{array}{l}24 \\
24\end{array}$ & $\begin{array}{l}5.1 \\
5.1\end{array}$ & $\begin{array}{l}10.6 \\
10.8\end{array}$ & $\begin{array}{l}2.2 \\
2.3\end{array}$ & $\begin{array}{l}1.9 \\
2.0\end{array}$ & $\begin{array}{l}19.6 \\
20.2\end{array}$ & $\begin{array}{l}17.5 \\
18.0\end{array}$ & $\begin{array}{l}22.1 \\
22.4\end{array}$ & $\begin{array}{l}0.07 \\
0.10\end{array}$ \\
\hline NGC 2701 & UI & $\begin{array}{l}16 \\
42\end{array}$ & $\begin{array}{l}37 \\
71\end{array}$ & $\begin{array}{l}42 \\
71\end{array}$ & $\begin{array}{l}71 \\
93\end{array}$ & $\begin{array}{l}38 \\
73\end{array}$ & $\begin{array}{r}6.7 \\
12.8\end{array}$ & $\begin{array}{r}24.1 \\
8.3\end{array}$ & $\begin{array}{l}4.2 \\
1.4\end{array}$ & $\begin{array}{l}2.9 \\
0.4\end{array}$ & $\begin{array}{l}20.2 \\
16.5\end{array}$ & $\begin{array}{l}16.5 \\
22.4\end{array}$ & $\begin{array}{l}22.1 \\
25.8\end{array}$ & $\begin{array}{l}0.05 \\
0.05\end{array}$ \\
\hline & II & $\begin{array}{l}16 \\
41\end{array}$ & $\begin{array}{l}37 \\
74\end{array}$ & $\begin{array}{l}41 \\
76\end{array}$ & $\begin{array}{r}74 \\
102\end{array}$ & $\begin{array}{l}38 \\
75\end{array}$ & $\begin{array}{r}6.7 \\
13.1\end{array}$ & $\begin{array}{r}27.8 \\
8.5\end{array}$ & $\begin{array}{l}4.9 \\
1.5\end{array}$ & $\begin{array}{l}3.3 \\
0.4\end{array}$ & $\begin{array}{l}20.7 \\
17.1\end{array}$ & $\begin{array}{l}17.1 \\
22.1\end{array}$ & $\begin{array}{l}21.9 \\
26.1\end{array}$ & $\begin{array}{l}0.07 \\
0.07\end{array}$ \\
\hline NGC 2776 & I & $\begin{array}{l}29 \\
29\end{array}$ & $\begin{array}{l}149 \\
134\end{array}$ & * & * & * & * & $\begin{array}{l}19.6 \\
20.1\end{array}$ & $\begin{array}{l}3.8 \\
3.9\end{array}$ & * & $\begin{array}{l}20.0 \\
20.5\end{array}$ & $\begin{array}{l}0.0 \\
0.0\end{array}$ & $\begin{array}{l}20.0 \\
20.5\end{array}$ & $\begin{array}{l}0.04 \\
0.05\end{array}$ \\
\hline NGC 2967 & III-d & $\begin{array}{l}10 \\
10\end{array}$ & $\begin{array}{l}59 \\
59\end{array}$ & $\begin{array}{l}90 \\
90\end{array}$ & $\begin{array}{l}156 \\
143\end{array}$ & $\begin{array}{l}73 \\
73\end{array}$ & $\begin{array}{l}9.4 \\
9.4\end{array}$ & $\begin{array}{l}16.3 \\
16.9\end{array}$ & $\begin{array}{l}2.1 \\
2.2\end{array}$ & $\begin{array}{l}0.5 \\
0.5\end{array}$ & $\begin{array}{l}19.4 \\
20.1\end{array}$ & $\begin{array}{l}22.0 \\
22.6\end{array}$ & $\begin{array}{l}24.8 \\
25.3\end{array}$ & $\begin{array}{l}0.28 \\
0.38\end{array}$ \\
\hline NGC 3055 & II.o-CT & $\begin{array}{l}10 \\
10\end{array}$ & $\begin{array}{l}53 \\
52\end{array}$ & $\begin{array}{l}57 \\
56\end{array}$ & $\begin{array}{l}88 \\
88\end{array}$ & $\begin{array}{l}54 \\
53\end{array}$ & $\begin{array}{l}6.8 \\
6.7\end{array}$ & $\begin{array}{l}17.1 \\
17.5\end{array}$ & $\begin{array}{l}2.1 \\
2.2\end{array}$ & $\begin{array}{l}1.9 \\
2.0\end{array}$ & $\begin{array}{l}19.6 \\
20.1\end{array}$ & $\begin{array}{l}16.3 \\
16.7\end{array}$ & $\begin{array}{l}23.1 \\
23.4\end{array}$ & $\begin{array}{l}0.14 \\
0.20\end{array}$ \\
\hline NGC 3246 & II-AB & $\begin{array}{l}7 \\
7\end{array}$ & $\begin{array}{l}59 \\
60\end{array}$ & $\begin{array}{l}64 \\
62\end{array}$ & $\begin{array}{l}96 \\
93\end{array}$ & $\begin{array}{l}61 \\
60\end{array}$ & $\begin{array}{l}9.0 \\
8.9\end{array}$ & $\begin{array}{l}20.2 \\
22.0\end{array}$ & $\begin{array}{l}3.0 \\
3.3\end{array}$ & $\begin{array}{l}2.0 \\
2.2\end{array}$ & $\begin{array}{l}20.8 \\
21.3\end{array}$ & $\begin{array}{l}17.1 \\
17.3\end{array}$ & $\begin{array}{l}24.3 \\
24.5\end{array}$ & $\begin{array}{l}0.10 \\
0.14\end{array}$ \\
\hline NGC 3259 & III-d & $\begin{array}{l}7 \\
7\end{array}$ & $\begin{array}{l}59 \\
59\end{array}$ & $\begin{array}{l}64 \\
63\end{array}$ & $\begin{array}{l}122 \\
138\end{array}$ & $\begin{array}{l}63 \\
63\end{array}$ & $\begin{array}{l}8.4 \\
8.4\end{array}$ & $\begin{array}{l}11.4 \\
12.2\end{array}$ & $\begin{array}{l}1.5 \\
1.6\end{array}$ & $\begin{array}{l}0.4 \\
0.4\end{array}$ & $\begin{array}{l}19.4 \\
20.0\end{array}$ & $\begin{array}{l}22.8 \\
23.1\end{array}$ & $\begin{array}{l}24.7 \\
25.0\end{array}$ & $\begin{array}{l}0.04 \\
0.05\end{array}$ \\
\hline NGC 3310 & III & $\begin{array}{l}10 \\
10\end{array}$ & $\begin{array}{l}52 \\
52\end{array}$ & $\begin{array}{l}53 \\
54\end{array}$ & $\begin{array}{l}172 \\
141\end{array}$ & $\begin{array}{l}53 \\
53\end{array}$ & $\begin{array}{l}4.4 \\
4.4\end{array}$ & $\begin{array}{l}10.6 \\
10.3\end{array}$ & $\begin{array}{l}0.9 \\
0.9\end{array}$ & $\begin{array}{l}0.3 \\
0.3\end{array}$ & $\begin{array}{l}18.2 \\
18.4\end{array}$ & $\begin{array}{l}21.6 \\
21.7\end{array}$ & $\begin{array}{l}23.2 \\
23.3\end{array}$ & $\begin{array}{l}0.06 \\
0.09\end{array}$ \\
\hline NGC 3359 & II-AB & $\begin{array}{l}29 \\
29\end{array}$ & $\begin{array}{l}183 \\
180\end{array}$ & $\begin{array}{l}204 \\
192\end{array}$ & $\begin{array}{l}266 \\
266\end{array}$ & $\begin{array}{l}184 \\
181\end{array}$ & $\begin{array}{l}16.1 \\
15.8\end{array}$ & $\begin{array}{l}51.1 \\
53.0\end{array}$ & $\begin{array}{l}4.5 \\
4.6\end{array}$ & $\begin{array}{l}1.8 \\
1.8\end{array}$ & $\begin{array}{l}20.5 \\
20.9\end{array}$ & $\begin{array}{l}16.9 \\
17.7\end{array}$ & $\begin{array}{l}24.9 \\
25.0\end{array}$ & $\begin{array}{l}0.03 \\
0.04\end{array}$ \\
\hline NGC 3423 & II-CT & $\begin{array}{l}30 \\
30\end{array}$ & $\begin{array}{l}76 \\
88\end{array}$ & $\begin{array}{l}93 \\
97\end{array}$ & $\begin{array}{l}175 \\
160\end{array}$ & $\begin{array}{l}85 \\
95\end{array}$ & $\begin{array}{l}6.1 \\
6.8\end{array}$ & $\begin{array}{l}52.2 \\
51.2\end{array}$ & $\begin{array}{l}3.7 \\
3.7\end{array}$ & $\begin{array}{l}2.8 \\
3.2\end{array}$ & $\begin{array}{l}20.9 \\
21.2\end{array}$ & $\begin{array}{l}17.7 \\
17.1\end{array}$ & $\begin{array}{l}22.6 \\
23.1\end{array}$ & $\begin{array}{l}0.08 \\
0.12\end{array}$ \\
\hline NGC 3488 & II.o-CT & $\begin{array}{l}10 \\
10\end{array}$ & $\begin{array}{l}32 \\
29\end{array}$ & $\begin{array}{l}52 \\
53\end{array}$ & $\begin{array}{l}70 \\
72\end{array}$ & $\begin{array}{l}44 \\
43\end{array}$ & $\begin{array}{l}9.8 \\
9.6\end{array}$ & $\begin{array}{l}16.8 \\
20.6\end{array}$ & $\begin{array}{l}3.8 \\
4.6\end{array}$ & $\begin{array}{l}2.5 \\
3.0\end{array}$ & $\begin{array}{l}20.3 \\
21.0\end{array}$ & $\begin{array}{l}16.0 \\
16.3\end{array}$ & $\begin{array}{l}23.2 \\
23.3\end{array}$ & $\begin{array}{l}0.04 \\
0.05\end{array}$ \\
\hline NGC 3583 & III-d & $\begin{array}{l}13 \\
13\end{array}$ & $\begin{array}{l}82 \\
81\end{array}$ & $\begin{array}{l}84 \\
82\end{array}$ & $\begin{array}{l}147 \\
138\end{array}$ & $\begin{array}{l}83 \\
82\end{array}$ & $\begin{array}{l}13.5 \\
13.3\end{array}$ & $\begin{array}{l}16.3 \\
17.0\end{array}$ & $\begin{array}{l}2.7 \\
2.8\end{array}$ & $\begin{array}{l}0.7 \\
0.7\end{array}$ & $\begin{array}{l}19.0 \\
19.7\end{array}$ & $\begin{array}{l}21.0 \\
21.4\end{array}$ & $\begin{array}{l}24.7 \\
25.7\end{array}$ & $\begin{array}{l}0.04 \\
0.05\end{array}$ \\
\hline
\end{tabular}


Table 3. continued.

\begin{tabular}{|c|c|c|c|c|c|c|c|c|c|c|c|c|c|c|}
\hline \multirow{3}{*}{$\begin{array}{l}\text { Galaxy } \\
\text { (1) }\end{array}$} & \multirow{3}{*}{$\begin{array}{l}\text { Break } \\
\text { type } \\
(2)\end{array}$} & \multirow{3}{*}{$\begin{array}{l}b_{1} \\
{\left[{ }^{\prime \prime}\right]}\end{array}$} & \multirow{3}{*}{$\begin{array}{l}b_{2} \\
{\left[{ }^{\prime \prime}\right]}\end{array}$} & \multirow{2}{*}{$\begin{array}{c}b_{3} \\
{\left[{ }^{\prime \prime}\right]}\end{array}$} & \multirow{3}{*}{$\begin{array}{c}b_{4} \\
{\left[{ }^{\prime \prime}\right]}\end{array}$} & \multicolumn{2}{|c|}{$R_{\mathrm{br}}$} & \multicolumn{2}{|c|}{$h_{\text {in }}$} & \multirow{3}{*}{$\begin{array}{c}h_{\text {in }} \\
{\left[h_{\text {out }}\right]} \\
(6)\end{array}$} & \multirow{3}{*}{$\begin{array}{c}\mu_{0, \text { in }} \\
{\left[\frac{\mathrm{mag}}{\square^{\prime \prime}}\right]}\end{array}$} & \multirow{3}{*}{$\begin{array}{l}\mu_{0, \text { out }} \\
{\left[\frac{\mathrm{mag}}{\mathrm{\sigma}^{\prime \prime}}\right]}\end{array}$} & \multirow{3}{*}{$\begin{array}{c}\mu_{\mathrm{br}} \\
{\left[\frac{\mathrm{mag}}{\mathrm{\sigma}^{\prime \prime}}\right]} \\
(8)\end{array}$} & \multirow{3}{*}{$\begin{array}{c}A_{r^{\prime} / g^{\prime}} \\
{[\mathrm{mag}]} \\
\quad(9)\end{array}$} \\
\hline & & & & & & {$\left[{ }^{\prime \prime}\right]$} & {$[\mathrm{kpc}]$} & {$\left[{ }^{\prime \prime}\right]$} & {$[\mathrm{kpc}]$} & & & & & \\
\hline & & & & (3) & & \multicolumn{2}{|c|}{ (4) } & \multicolumn{2}{|c|}{ (5) } & & & & & \\
\hline NGC 3589 & II-CT & $\begin{array}{l}0 \\
0\end{array}$ & $\begin{array}{l}37 \\
33\end{array}$ & $\begin{array}{l}38 \\
38\end{array}$ & $\begin{array}{l}72 \\
84\end{array}$ & $\begin{array}{l}38 \\
36\end{array}$ & $\begin{array}{l}5.8 \\
5.5\end{array}$ & $\begin{array}{l}18.6 \\
20.5\end{array}$ & $\begin{array}{l}2.9 \\
3.1\end{array}$ & $\begin{array}{l}1.7 \\
1.8\end{array}$ & $\begin{array}{l}21.1 \\
21.5\end{array}$ & $\begin{array}{l}19.7 \\
20.3\end{array}$ & $\begin{array}{l}23.1 \\
23.0\end{array}$ & $\begin{array}{l}0.03 \\
0.04\end{array}$ \\
\hline UGC 06309 & II-i & $\begin{array}{l}23 \\
25\end{array}$ & $\begin{array}{l}74 \\
88\end{array}$ & * & * & $*$ & $*$ & $\begin{array}{r}9.8 \\
11.1\end{array}$ & $\begin{array}{l}2.1 \\
2.4\end{array}$ & * & $\begin{array}{l}18.9 \\
19.9\end{array}$ & $\begin{array}{l}0.0 \\
0.0\end{array}$ & $\begin{array}{l}18.9 \\
19.9\end{array}$ & $\begin{array}{l}0.05 \\
0.07\end{array}$ \\
\hline NGC 3631 & I & $\begin{array}{l}140 \\
140\end{array}$ & $\begin{array}{l}233 \\
226\end{array}$ & $\begin{array}{l}* \\
*\end{array}$ & $\begin{array}{l}* \\
*\end{array}$ & $\begin{array}{l}* \\
*\end{array}$ & $\begin{array}{l}* \\
*\end{array}$ & $\begin{array}{l}37.8 \\
36.7\end{array}$ & $\begin{array}{l}3.6 \\
3.5\end{array}$ & $\begin{array}{l}* \\
*\end{array}$ & $\begin{array}{l}20.8 \\
21.2\end{array}$ & $\begin{array}{l}0.0 \\
0.0\end{array}$ & $\begin{array}{l}20.8 \\
21.2\end{array}$ & $\begin{array}{l}0.05 \\
0.06\end{array}$ \\
\hline NGC 3642 & III-d & $\begin{array}{l}30 \\
30\end{array}$ & $\begin{array}{l}75 \\
71\end{array}$ & $\begin{array}{l}79 \\
73\end{array}$ & $\begin{array}{l}229 \\
236\end{array}$ & $\begin{array}{l}79 \\
73\end{array}$ & $\begin{array}{r}10.0 \\
9.3\end{array}$ & $\begin{array}{l}20.9 \\
19.7\end{array}$ & $\begin{array}{l}2.6 \\
2.5\end{array}$ & $\begin{array}{l}0.5 \\
0.4\end{array}$ & $\begin{array}{l}20.3 \\
20.6\end{array}$ & $\begin{array}{l}22.3 \\
22.7\end{array}$ & $\begin{array}{l}24.0 \\
24.3\end{array}$ & $\begin{array}{l}0.03 \\
0.04\end{array}$ \\
\hline UGC 06518 & II-i & $\begin{array}{l}10 \\
10\end{array}$ & $\begin{array}{l}43 \\
45\end{array}$ & * & $\begin{array}{l}* \\
*\end{array}$ & * & $\begin{array}{l}* \\
*\end{array}$ & $\begin{array}{l}6.4 \\
6.1\end{array}$ & $\begin{array}{l}1.4 \\
1.3\end{array}$ & $\begin{array}{l}* \\
*\end{array}$ & $\begin{array}{l}19.3 \\
19.6\end{array}$ & $\begin{array}{l}0.0 \\
0.0\end{array}$ & $\begin{array}{l}19.3 \\
19.6\end{array}$ & $\begin{array}{l}0.04 \\
0.05\end{array}$ \\
\hline NGC 3756 & II.o-CT & $\begin{array}{l}11 \\
11\end{array}$ & $\begin{array}{l}92 \\
78\end{array}$ & $\begin{array}{l}103 \\
104\end{array}$ & $\begin{array}{l}154 \\
149\end{array}$ & $\begin{array}{r}97 \\
100\end{array}$ & $\begin{array}{l}10.2 \\
10.6\end{array}$ & $\begin{array}{l}33.7 \\
38.6\end{array}$ & $\begin{array}{l}3.6 \\
4.1\end{array}$ & $\begin{array}{l}2.0 \\
2.2\end{array}$ & $\begin{array}{l}20.0 \\
20.8\end{array}$ & $\begin{array}{l}16.6 \\
17.7\end{array}$ & $\begin{array}{l}23.2 \\
23.4\end{array}$ & $\begin{array}{l}0.03 \\
0.04\end{array}$ \\
\hline NGC 3888 & I & $\begin{array}{l}13 \\
13\end{array}$ & $\begin{array}{l}81 \\
84\end{array}$ & $\begin{array}{l}* \\
*\end{array}$ & $*$ & * & $\begin{array}{l}* \\
*\end{array}$ & $\begin{array}{r}10.1 \\
9.9\end{array}$ & $\begin{array}{l}1.9 \\
1.8\end{array}$ & * & $\begin{array}{l}18.7 \\
19.1\end{array}$ & $\begin{array}{l}0.0 \\
0.0\end{array}$ & $\begin{array}{l}18.7 \\
19.1\end{array}$ & $\begin{array}{l}0.03 \\
0.04\end{array}$ \\
\hline NGC 3893 & III-d & $\begin{array}{l}15 \\
15\end{array}$ & $\begin{array}{l}134 \\
139\end{array}$ & $\begin{array}{l}134 \\
139\end{array}$ & $\begin{array}{l}184 \\
184\end{array}$ & $\begin{array}{l}134 \\
139\end{array}$ & $\begin{array}{l}11.1 \\
11.5\end{array}$ & $\begin{array}{l}29.1 \\
28.3\end{array}$ & $\begin{array}{l}2.4 \\
2.3\end{array}$ & $\begin{array}{l}0.6 \\
0.5\end{array}$ & $\begin{array}{l}19.2 \\
19.6\end{array}$ & $\begin{array}{l}21.7 \\
22.6\end{array}$ & $\begin{array}{l}24.7 \\
25.5\end{array}$ & $\begin{array}{l}0.06 \\
0.08\end{array}$ \\
\hline UGC 06903 & II.o-CT & $\begin{array}{l}21 \\
21\end{array}$ & $\begin{array}{l}59 \\
56\end{array}$ & $\begin{array}{l}72 \\
63\end{array}$ & $\begin{array}{l}98 \\
92\end{array}$ & $\begin{array}{l}60 \\
57\end{array}$ & $\begin{array}{l}8.0 \\
7.6\end{array}$ & $\begin{array}{l}31.8 \\
35.0\end{array}$ & $\begin{array}{l}4.2 \\
4.6\end{array}$ & $\begin{array}{l}3.6 \\
3.6\end{array}$ & $\begin{array}{l}21.6 \\
22.2\end{array}$ & $\begin{array}{l}15.8 \\
17.1\end{array}$ & $\begin{array}{l}23.9 \\
24.2\end{array}$ & $\begin{array}{l}0.06 \\
0.08\end{array}$ \\
\hline NGC 3982 & III & $\begin{array}{l}13 \\
13\end{array}$ & $\begin{array}{l}51 \\
49\end{array}$ & $\begin{array}{l}54 \\
51\end{array}$ & $\begin{array}{l}91 \\
99\end{array}$ & $\begin{array}{l}52 \\
50\end{array}$ & $\begin{array}{l}4.9 \\
4.7\end{array}$ & $\begin{array}{l}10.2 \\
10.2\end{array}$ & $\begin{array}{l}1.0 \\
1.0\end{array}$ & $\begin{array}{l}0.7 \\
0.8\end{array}$ & $\begin{array}{l}18.3 \\
18.7\end{array}$ & $\begin{array}{l}19.8 \\
19.8\end{array}$ & $\begin{array}{l}23.8 \\
23.6\end{array}$ & $\begin{array}{l}0.04 \\
0.05\end{array}$ \\
\hline NGC 3992 & II.o-OLR & $\begin{array}{l}44 \\
44\end{array}$ & $\begin{array}{l}133 \\
129\end{array}$ & $\begin{array}{l}139 \\
133\end{array}$ & $\begin{array}{l}324 \\
280\end{array}$ & $\begin{array}{l}137 \\
133\end{array}$ & $\begin{array}{l}12.2 \\
11.8\end{array}$ & $\begin{array}{l}84.0 \\
96.4\end{array}$ & $\begin{array}{l}7.5 \\
8.6\end{array}$ & $\begin{array}{l}2.3 \\
2.7\end{array}$ & $\begin{array}{l}20.5 \\
21.3\end{array}$ & $\begin{array}{l}18.1 \\
18.4\end{array}$ & $\begin{array}{l}22.3 \\
22.9\end{array}$ & $\begin{array}{l}0.08 \\
0.12\end{array}$ \\
\hline NGC 4030 & III & $\begin{array}{l}37 \\
37\end{array}$ & $\begin{array}{l}147 \\
147\end{array}$ & $\begin{array}{l}164 \\
151\end{array}$ & $\begin{array}{l}266 \\
230\end{array}$ & $\begin{array}{l}152 \\
148\end{array}$ & $\begin{array}{l}15.5 \\
15.1\end{array}$ & $\begin{array}{l}26.2 \\
25.5\end{array}$ & $\begin{array}{l}2.7 \\
2.6\end{array}$ & $\begin{array}{l}0.5 \\
0.6\end{array}$ & $\begin{array}{l}19.2 \\
19.6\end{array}$ & $\begin{array}{l}22.1 \\
22.3\end{array}$ & $\begin{array}{l}25.4 \\
26.0\end{array}$ & $\begin{array}{l}0.08 \\
0.11\end{array}$ \\
\hline NGC 4041 & III-d & $\begin{array}{l}26 \\
26\end{array}$ & $\begin{array}{l}76 \\
81\end{array}$ & $\begin{array}{l}80 \\
84\end{array}$ & $\begin{array}{l}192 \\
186\end{array}$ & $\begin{array}{l}80 \\
83\end{array}$ & $\begin{array}{l}8.2 \\
8.5\end{array}$ & $\begin{array}{l}18.6 \\
18.2\end{array}$ & $\begin{array}{l}1.9 \\
1.9\end{array}$ & $\begin{array}{l}0.6 \\
0.5\end{array}$ & $\begin{array}{l}19.7 \\
20.1\end{array}$ & $\begin{array}{l}21.8 \\
22.6\end{array}$ & $\begin{array}{l}24.6 \\
25.2\end{array}$ & $\begin{array}{l}0.05 \\
0.07\end{array}$ \\
\hline NGC 4102 & II.o-OLR + III & $\begin{array}{l}40 \\
57\end{array}$ & $\begin{array}{r}57 \\
116\end{array}$ & $\begin{array}{r}57 \\
119\end{array}$ & $\begin{array}{l}116 \\
165\end{array}$ & $\begin{array}{r}57 \\
118\end{array}$ & $\begin{array}{l}4.3 \\
8.8\end{array}$ & $\begin{array}{l}23.3 \\
15.8\end{array}$ & $\begin{array}{l}1.7 \\
1.2\end{array}$ & $\begin{array}{l}1.5 \\
0.6\end{array}$ & $\begin{array}{l}19.1 \\
17.8\end{array}$ & $\begin{array}{l}17.8 \\
20.9\end{array}$ & $\begin{array}{l}21.7 \\
26.0\end{array}$ & $\begin{array}{l}0.06 \\
0.06\end{array}$ \\
\hline 1004102 & 11.0-ULN + III & $\begin{array}{l}40 \\
59\end{array}$ & $\begin{array}{r}59 \\
107\end{array}$ & $\begin{array}{r}59 \\
109\end{array}$ & $\begin{array}{l}107 \\
151\end{array}$ & $\begin{array}{r}59 \\
108\end{array}$ & $\begin{array}{l}4.4 \\
8.1\end{array}$ & $\begin{array}{l}21.6 \\
15.7\end{array}$ & $\begin{array}{l}1.6 \\
1.2\end{array}$ & $\begin{array}{l}1.4 \\
0.7\end{array}$ & $\begin{array}{l}19.5 \\
18.4\end{array}$ & $\begin{array}{l}18.4 \\
20.4\end{array}$ & $\begin{array}{l}22.4 \\
26.1\end{array}$ & $\begin{array}{l}0.08 \\
0.08\end{array}$ \\
\hline NGC 4108 & II-AB & $\begin{array}{l}7 \\
7\end{array}$ & $\begin{array}{l}44 \\
43\end{array}$ & $\begin{array}{l}46 \\
45\end{array}$ & $\begin{array}{l}66 \\
68\end{array}$ & $\begin{array}{l}44 \\
44\end{array}$ & $\begin{array}{l}8.6 \\
8.6\end{array}$ & $\begin{array}{l}9.1 \\
9.8\end{array}$ & $\begin{array}{l}1.8 \\
1.9\end{array}$ & $\begin{array}{l}1.5 \\
1.6\end{array}$ & $\begin{array}{l}18.9 \\
19.5\end{array}$ & $\begin{array}{l}16.2 \\
16.5\end{array}$ & $\begin{array}{l}24.5 \\
24.5\end{array}$ & $\begin{array}{l}0.05 \\
0.07\end{array}$ \\
\hline NGC 4108B & I & $\begin{array}{l}7 \\
7\end{array}$ & $\begin{array}{l}67 \\
71\end{array}$ & * & * & * & $*$ & $\begin{array}{l}11.4 \\
11.0\end{array}$ & 2.2 & * & $\begin{array}{l}20.8 \\
21.0\end{array}$ & $\begin{array}{l}0.0 \\
0.0\end{array}$ & $\begin{array}{l}20.8 \\
21.0\end{array}$ & $\begin{array}{l}0.05 \\
0.07\end{array}$ \\
\hline NGC 4123 & I & $\begin{array}{l}20 \\
20\end{array}$ & $\begin{array}{l}220 \\
207\end{array}$ & * & * & $\begin{array}{l}* \\
*\end{array}$ & $\begin{array}{l}* \\
*\end{array}$ & $\begin{array}{l}35.5 \\
36.5\end{array}$ & $\begin{array}{l}3.4 \\
3.5\end{array}$ & $\begin{array}{l}* \\
*\end{array}$ & $\begin{array}{l}20.6 \\
21.2\end{array}$ & $\begin{array}{l}0.0 \\
0.0\end{array}$ & $\begin{array}{l}20.6 \\
21.2\end{array}$ & $\begin{array}{l}0.06 \\
0.08\end{array}$ \\
\hline NGC 4210 & II.o-OLR + CT & $\begin{array}{l}15 \\
37\end{array}$ & $\begin{array}{l}35 \\
50\end{array}$ & $\begin{array}{l}37 \\
55\end{array}$ & $\begin{array}{l}50 \\
76\end{array}$ & $\begin{array}{l}36 \\
51\end{array}$ & $\begin{array}{r}7.5 \\
10.6\end{array}$ & $\begin{array}{l}16.3 \\
10.2\end{array}$ & $\begin{array}{l}3.4 \\
2.1\end{array}$ & $\begin{array}{l}1.6 \\
1.5\end{array}$ & $\begin{array}{l}19.9 \\
18.6\end{array}$ & $\begin{array}{l}18.6 \\
15.6\end{array}$ & $\begin{array}{l}22.1 \\
24.1\end{array}$ & $\begin{array}{l}0.05 \\
0.05\end{array}$ \\
\hline & & $\begin{array}{l}15 \\
38\end{array}$ & $\begin{array}{l}32 \\
50\end{array}$ & $\begin{array}{l}38 \\
52\end{array}$ & $\begin{array}{l}50 \\
73\end{array}$ & $\begin{array}{l}37 \\
51\end{array}$ & $\begin{array}{r}7.7 \\
10.6\end{array}$ & $\begin{array}{l}19.5 \\
10.0\end{array}$ & $\begin{array}{l}4.1 \\
2.1\end{array}$ & $\begin{array}{l}2.0 \\
1.6\end{array}$ & $\begin{array}{l}20.7 \\
19.0\end{array}$ & $\begin{array}{l}19.0 \\
15.6\end{array}$ & $\begin{array}{l}22.4 \\
24.9\end{array}$ & $\begin{array}{l}0.07 \\
0.07\end{array}$ \\
\hline NGC 4273 & II- & $\begin{array}{l}12 \\
48\end{array}$ & $\begin{array}{l}43 \\
78\end{array}$ & $\begin{array}{l}48 \\
79\end{array}$ & $\begin{array}{r}78 \\
119\end{array}$ & $\begin{array}{l}47 \\
78\end{array}$ & $\begin{array}{r}7.9 \\
13.2\end{array}$ & $\begin{array}{l}24.5 \\
11.5\end{array}$ & $\begin{array}{l}4.1 \\
1.9\end{array}$ & $\begin{array}{l}2.1 \\
0.6\end{array}$ & $\begin{array}{l}19.8 \\
17.6\end{array}$ & $\begin{array}{l}17.6 \\
20.3\end{array}$ & $\begin{array}{l}21.7 \\
25.4\end{array}$ & $\begin{array}{l}0.05 \\
0.05\end{array}$ \\
\hline NGC 4213 & II & $\begin{array}{l}12 \\
47\end{array}$ & $\begin{array}{l}45 \\
84\end{array}$ & $\begin{array}{l}47 \\
89\end{array}$ & $\begin{array}{r}84 \\
106\end{array}$ & $\begin{array}{l}47 \\
84\end{array}$ & $\begin{array}{r}7.9 \\
14.2\end{array}$ & $\begin{array}{l}29.7 \\
11.2\end{array}$ & $\begin{array}{l}5.0 \\
1.9\end{array}$ & $\begin{array}{l}2.6 \\
0.7\end{array}$ & $\begin{array}{l}20.5 \\
17.9\end{array}$ & $\begin{array}{l}17.9 \\
20.0\end{array}$ & $\begin{array}{l}22.1 \\
25.6\end{array}$ & $\begin{array}{l}0.07 \\
0.07\end{array}$ \\
\hline NGC 4480 & II.o-CT & $\begin{array}{l}8 \\
8\end{array}$ & $\begin{array}{l}53 \\
54\end{array}$ & $\begin{array}{l}58 \\
57\end{array}$ & $\begin{array}{l}90 \\
88\end{array}$ & $\begin{array}{l}54 \\
54\end{array}$ & $\begin{array}{l}9.3 \\
9.3\end{array}$ & $\begin{array}{l}18.8 \\
21.4\end{array}$ & $\begin{array}{l}3.2 \\
3.7\end{array}$ & $\begin{array}{l}2.3 \\
2.6\end{array}$ & $\begin{array}{l}19.8 \\
20.5\end{array}$ & $\begin{array}{l}15.8 \\
16.1\end{array}$ & $\begin{array}{l}23.0 \\
23.3\end{array}$ & $\begin{array}{l}0.06 \\
0.09\end{array}$ \\
\hline & & $\begin{array}{l}25 \\
77\end{array}$ & $\begin{array}{r}76 \\
139\end{array}$ & $\begin{array}{r}77 \\
159\end{array}$ & $\begin{array}{l}139 \\
192\end{array}$ & $\begin{array}{r}77 \\
140\end{array}$ & $\begin{array}{r}8.3 \\
15.1\end{array}$ & $\begin{array}{l}53.3 \\
39.7\end{array}$ & $\begin{array}{l}5.8 \\
4.3\end{array}$ & $\begin{array}{l}1.3 \\
2.3\end{array}$ & $\begin{array}{l}22.0 \\
21.4\end{array}$ & $\begin{array}{l}21.4 \\
15.7\end{array}$ & $\begin{array}{l}23.6 \\
25.7\end{array}$ & $\begin{array}{l}0.06 \\
0.06\end{array}$ \\
\hline$N G$ & & $\begin{array}{l}25 \\
77\end{array}$ & $\begin{array}{r}76 \\
139\end{array}$ & $\begin{array}{r}77 \\
159\end{array}$ & $\begin{array}{l}139 \\
192\end{array}$ & $\begin{array}{r}77 \\
140\end{array}$ & $\begin{array}{r}8.3 \\
15.1\end{array}$ & $\begin{array}{l}59.3 \\
41.1\end{array}$ & $\begin{array}{l}6.4 \\
4.4\end{array}$ & $\begin{array}{l}1.4 \\
2.5\end{array}$ & $\begin{array}{l}22.5 \\
21.8\end{array}$ & $\begin{array}{l}21.8 \\
15.5\end{array}$ & $\begin{array}{l}23.9 \\
26.0\end{array}$ & $\begin{array}{l}0.09 \\
0.09\end{array}$ \\
\hline UGC 07700 & II.o-CT & $\begin{array}{l}12 \\
12\end{array}$ & $\begin{array}{l}45 \\
49\end{array}$ & $\begin{array}{l}52 \\
58\end{array}$ & $\begin{array}{l}94 \\
74\end{array}$ & $\begin{array}{l}50 \\
50\end{array}$ & $\begin{array}{l}11.2 \\
11.2\end{array}$ & $\begin{array}{l}26.4 \\
29.1\end{array}$ & $\begin{array}{l}5.9 \\
6.5\end{array}$ & $\begin{array}{l}2.2 \\
3.7\end{array}$ & $\begin{array}{l}22.2 \\
22.6\end{array}$ & $\begin{array}{l}20.2 \\
17.5\end{array}$ & $\begin{array}{l}23.8 \\
24.5\end{array}$ & $\begin{array}{l}0.05 \\
0.07\end{array}$ \\
\hline NGC 4545 & II.o-CT & $\begin{array}{l}7 \\
7\end{array}$ & $\begin{array}{l}56 \\
49\end{array}$ & $\begin{array}{l}58 \\
57\end{array}$ & $\begin{array}{l}97 \\
89\end{array}$ & $\begin{array}{l}56 \\
55\end{array}$ & $\begin{array}{l}11.6 \\
11.4\end{array}$ & $\begin{array}{l}17.7 \\
19.2\end{array}$ & $\begin{array}{l}3.7 \\
4.0\end{array}$ & $\begin{array}{l}2.0 \\
2.1\end{array}$ & $\begin{array}{l}20.0 \\
20.6\end{array}$ & $\begin{array}{l}16.3 \\
16.7\end{array}$ & $\begin{array}{l}23.8 \\
24.0\end{array}$ & $\begin{array}{l}0.03 \\
0.04\end{array}$ \\
\hline NGC 4653 & II-AB & $\begin{array}{l}11 \\
11\end{array}$ & $\begin{array}{l}102 \\
101\end{array}$ & $\begin{array}{l}109 \\
107\end{array}$ & $\begin{array}{l}142 \\
130\end{array}$ & $\begin{array}{l}103 \\
102\end{array}$ & $\begin{array}{l}19.0 \\
18.8\end{array}$ & $\begin{array}{l}23.0 \\
24.5\end{array}$ & $\begin{array}{l}4.2 \\
4.5\end{array}$ & $\begin{array}{l}1.7 \\
1.8\end{array}$ & $\begin{array}{l}20.7 \\
21.2\end{array}$ & $\begin{array}{l}17.1 \\
17.1\end{array}$ & $\begin{array}{l}25.9 \\
26.0\end{array}$ & $\begin{array}{l}0.06 \\
0.09\end{array}$ \\
\hline
\end{tabular}


Table 3. continued.

\begin{tabular}{|c|c|c|c|c|c|c|c|c|c|c|c|c|c|c|}
\hline \multirow{3}{*}{$\begin{array}{l}\text { Galaxy } \\
\text { (1) }\end{array}$} & \multirow{2}{*}{$\begin{array}{c}\text { Break } \\
\text { type }\end{array}$} & \multirow{2}{*}{$\begin{array}{c}b_{1} \\
{\left[{ }^{\prime \prime}\right]}\end{array}$} & \multirow{2}{*}{$\begin{array}{c}b_{2} \\
{\left[{ }^{\prime \prime}\right]}\end{array}$} & \multirow{2}{*}{$\begin{array}{c}b_{3} \\
{\left[{ }^{\prime \prime}\right]}\end{array}$} & \multirow{2}{*}{$\begin{array}{c}b_{4} \\
{\left[{ }^{\prime \prime}\right]}\end{array}$} & \multicolumn{2}{|c|}{$R_{\mathrm{br}}$} & \multicolumn{2}{|c|}{$h_{\text {in }}$} & \multirow{3}{*}{$\begin{array}{c}h_{\text {in }} \\
{\left[h_{\text {out }}\right]} \\
(6)\end{array}$} & \multirow{2}{*}{$\begin{array}{c}\mu_{0, \text { in }} \\
{\left[\frac{\mathrm{mag}}{\square^{\prime \prime}}\right]}\end{array}$} & \multirow{2}{*}{$\begin{array}{l}\mu_{0, \text { out }} \\
{\left[\frac{\mathrm{mag}}{\mathrm{a}^{\prime \prime}}\right]}\end{array}$} & \multirow{2}{*}{$\begin{array}{c}\mu_{\mathrm{br}} \\
{\left[\frac{\mathrm{mag}}{\square^{\prime \prime}}\right]}\end{array}$} & \multirow{2}{*}{$\begin{array}{c}A_{r^{\prime} / g^{\prime}} \\
{[\mathrm{mag}]}\end{array}$} \\
\hline & & & & & & {$\left[{ }^{\prime \prime}\right]$} & [kpc] & {$\left[{ }^{\prime \prime}\right]$} & [kpc] & & & & & \\
\hline & (2) & \multicolumn{4}{|c|}{ (3) } & \multicolumn{2}{|c|}{ (4) } & \multicolumn{2}{|c|}{ (5) } & & \multicolumn{2}{|c|}{ (7) } & $(8)$ & (9) \\
\hline NGC 4668 & III & $\begin{array}{l}0 \\
0\end{array}$ & $\begin{array}{l}60 \\
65\end{array}$ & $\begin{array}{l}71 \\
71\end{array}$ & $\begin{array}{l}110 \\
104\end{array}$ & $\begin{array}{l}66 \\
68\end{array}$ & $\begin{array}{l}7.6 \\
7.8\end{array}$ & $\begin{array}{l}11.8 \\
11.7\end{array}$ & $\begin{array}{l}1.4 \\
1.3\end{array}$ & $\begin{array}{l}0.5 \\
0.5\end{array}$ & $\begin{array}{l}19.6 \\
20.0\end{array}$ & $\begin{array}{l}22.2 \\
23.2\end{array}$ & $\begin{array}{l}25.5 \\
25.9\end{array}$ & $\begin{array}{l}0.07 \\
0.10\end{array}$ \\
\hline UGC 08041 & II.o-CT & $\begin{array}{l}14 \\
14\end{array}$ & $\begin{array}{l}69 \\
69\end{array}$ & $\begin{array}{l}73 \\
75\end{array}$ & $\begin{array}{l}148 \\
157\end{array}$ & $\begin{array}{l}71 \\
70\end{array}$ & $\begin{array}{l}6.8 \\
6.7\end{array}$ & $\begin{array}{l}36.9 \\
39.9\end{array}$ & $\begin{array}{l}3.5 \\
3.8\end{array}$ & $\begin{array}{l}1.4 \\
1.6\end{array}$ & $\begin{array}{l}21.1 \\
21.6\end{array}$ & $\begin{array}{l}20.2 \\
20.5\end{array}$ & $\begin{array}{l}23.1 \\
23.4\end{array}$ & $\begin{array}{l}0.06 \\
0.09\end{array}$ \\
\hline UGC 08084 & II.o-OLR & $\begin{array}{l}9 \\
9\end{array}$ & $\begin{array}{l}38 \\
35\end{array}$ & $\begin{array}{l}44 \\
45\end{array}$ & $\begin{array}{l}71 \\
78\end{array}$ & $\begin{array}{l}42 \\
43\end{array}$ & $\begin{array}{l}8.2 \\
8.4\end{array}$ & $\begin{array}{l}29.0 \\
31.3\end{array}$ & $\begin{array}{l}5.7 \\
6.1\end{array}$ & $\begin{array}{l}3.4 \\
3.9\end{array}$ & 22.4 & $\begin{array}{l}18.8 \\
18.7\end{array}$ & $\begin{array}{l}23.9 \\
24.2\end{array}$ & $\begin{array}{l}0.08 \\
0.11\end{array}$ \\
\hline NGC 4004 & U & $\begin{array}{l}13 \\
48\end{array}$ & $\begin{array}{r}38 \\
103\end{array}$ & $\begin{array}{r}48 \\
116\end{array}$ & $\begin{array}{l}103 \\
157\end{array}$ & $\begin{array}{r}39 \\
104\end{array}$ & $\begin{array}{l}3.3 \\
8.7\end{array}$ & 27.6 & $\begin{array}{l}2.3 \\
10\end{array}$ & 2.2 & 20.2 & 18.2 & 21.9 & 0.07 \\
\hline NGC 4904 & 1 & 13 & 40 & 40 & 99 & 40 & 3.4 & 32.7 & 2.7 & 2.6 & 20.9 & 18.8 & 22.2 & 0.10 \\
\hline & & & 99 & 99 & 135 & 99 & 8.3 & 12.7 & 1.1 & 0.3 & 18.8 & 25.1 & 27.4 & 0.10 \\
\hline UGC 08237 & II.o-OLR & $\begin{array}{l}14 \\
14\end{array}$ & $\begin{array}{l}25 \\
27\end{array}$ & $\begin{array}{l}29 \\
31\end{array}$ & $\begin{array}{l}50 \\
48\end{array}$ & $\begin{array}{l}26 \\
30\end{array}$ & $\begin{array}{l}5.6 \\
6.5\end{array}$ & $\begin{array}{l}14.8 \\
14.0\end{array}$ & $\begin{array}{l}3.2 \\
3.0\end{array}$ & $\begin{array}{l}2.6 \\
2.5\end{array}$ & $\begin{array}{l}20.6 \\
21.1\end{array}$ & $\begin{array}{l}17.8 \\
18.4\end{array}$ & $\begin{array}{l}22.4 \\
23.0\end{array}$ & $\begin{array}{l}0.05 \\
0.06\end{array}$ \\
\hline & & $\begin{array}{r}5 \\
32\end{array}$ & $\begin{array}{l}29 \\
72\end{array}$ & $\begin{array}{l}32 \\
77\end{array}$ & $\begin{array}{r}72 \\
122\end{array}$ & $\begin{array}{l}30 \\
73\end{array}$ & $\begin{array}{l}2.4 \\
5.8\end{array}$ & $\begin{array}{l}20.3 \\
11.0\end{array}$ & $\begin{array}{l}1.6 \\
0.9\end{array}$ & $\begin{array}{l}1.8 \\
0.5\end{array}$ & $\begin{array}{l}19.8 \\
18.3\end{array}$ & $\begin{array}{l}18.3 \\
22.0\end{array}$ & $\begin{array}{l}21.7 \\
25.8\end{array}$ & $\begin{array}{l}0.08 \\
0.08\end{array}$ \\
\hline NGC 5147 & II.o-OLR + III & $\begin{array}{r}32 \\
5\end{array}$ & 26 & 29 & 75 & 29 & 23 & 22.8 & 1.8 & 2.2 & 20.4 & 18.5 & 25.0 & 0.08 \\
\hline & & 29 & 75 & 79 & 108 & 76 & 6.1 & 10.5 & $\begin{array}{l}1.8 \\
0.8\end{array}$ & 0.6 & 18.5 & 21.6 & 26.2 & 0.11 \\
\hline UGC 08658 & II-CT & $\begin{array}{l}12 \\
12\end{array}$ & $\begin{array}{l}57 \\
57\end{array}$ & $\begin{array}{l}61 \\
61\end{array}$ & $\begin{array}{l}102 \\
122\end{array}$ & $\begin{array}{l}61 \\
61\end{array}$ & $\begin{array}{l}9.7 \\
9.7\end{array}$ & $\begin{array}{l}22.4 \\
24.4\end{array}$ & $\begin{array}{l}3.5 \\
3.9\end{array}$ & $\begin{array}{l}1.4 \\
1.5\end{array}$ & $\begin{array}{l}21.0 \\
21.6\end{array}$ & $\begin{array}{l}20.0 \\
20.3\end{array}$ & $\begin{array}{l}23.9 \\
24.4\end{array}$ & $\begin{array}{l}0.05 \\
0.06\end{array}$ \\
\hline NGC 5300 & II.o-CT & $\begin{array}{l}12 \\
12\end{array}$ & $\begin{array}{l}80 \\
75\end{array}$ & $\begin{array}{l}85 \\
82\end{array}$ & $\begin{array}{l}155 \\
142\end{array}$ & $\begin{array}{l}83 \\
77\end{array}$ & $\begin{array}{l}7.2 \\
6.6\end{array}$ & $\begin{array}{l}37.1 \\
41.8\end{array}$ & $\begin{array}{l}3.2 \\
3.6\end{array}$ & $\begin{array}{l}2.1 \\
2.6\end{array}$ & $\begin{array}{l}20.8 \\
21.4\end{array}$ & $\begin{array}{l}18.2 \\
18.1\end{array}$ & $\begin{array}{l}23.2 \\
23.5\end{array}$ & $\begin{array}{l}0.06 \\
0.09\end{array}$ \\
\hline NGC 5334 & II.o-CT & 13 & 80 & 102 & 152 & 92 & 9.1 & 40.2 & 4.0 & 2.6 & 21.0 & 16.7 & 23.8 & 0.13 \\
\hline & & 13 & 101 & 103 & 156 & 103 & 10.2 & 40.3 & 4.0 & 2.8 & 21.5 & 16.4 & 24.4 & 0.18 \\
\hline NGC 5376 & II.o-OLR & $\begin{array}{l}10 \\
10\end{array}$ & $\begin{array}{l}34 \\
34\end{array}$ & $\begin{array}{l}35 \\
39\end{array}$ & $\begin{array}{l}97 \\
95\end{array}$ & $\begin{array}{l}35 \\
37\end{array}$ & $\begin{array}{l}5.6 \\
5.9\end{array}$ & $\begin{array}{l}17.0 \\
17.9\end{array}$ & $\begin{array}{l}2.7 \\
2.8\end{array}$ & $\begin{array}{l}1.4 \\
1.7\end{array}$ & $\begin{array}{l}19.4 \\
20.1\end{array}$ & $\begin{array}{l}18.6 \\
18.7\end{array}$ & 21.2 & $\begin{array}{l}0.04 \\
0.05\end{array}$ \\
\hline NGC 5430 & II.o-OLR & 10 & 69 & 72 & 105 & 70 & 15.7 & 15.8 & 3.5 & 1.4 & 19.4 & 17.8 & 23.9 & 0.05 \\
\hline & & 10 & 69 & 71 & 102 & 69 & 15.5 & 16.2 & 3.6 & 1.6 & 20.1 & 17.6 & 24.4 & 0.06 \\
\hline NGC 5480 & III-d & $\begin{array}{l}9 \\
9\end{array}$ & $\begin{array}{l}65 \\
67\end{array}$ & $\begin{array}{l}74 \\
76\end{array}$ & $\begin{array}{l}129 \\
122\end{array}$ & $\begin{array}{l}67 \\
68\end{array}$ & $\begin{array}{r}9.8 \\
10.0\end{array}$ & $\begin{array}{l}13.9 \\
13.5\end{array}$ & $\begin{array}{l}2.0 \\
2.0\end{array}$ & $\begin{array}{l}0.5 \\
0.5\end{array}$ & $\begin{array}{l}19.6 \\
20.1\end{array}$ & $\begin{array}{l}22.1 \\
22.6\end{array}$ & $\begin{array}{l}24.9 \\
25.4\end{array}$ & $\begin{array}{l}0.05 \\
0.07\end{array}$ \\
\hline NGC 5584 & II.o-CT & 14 & $\begin{array}{l}75 \\
68\end{array}$ & $\begin{array}{r}107 \\
92\end{array}$ & 165 & $\begin{array}{l}90 \\
84\end{array}$ & $\begin{array}{r}10.6 \\
99\end{array}$ & $\begin{array}{l}39.8 \\
45.4\end{array}$ & $\begin{array}{l}4.7 \\
54\end{array}$ & 2.0 & 20.9 & 18.4 & 23.3 & 0.11 \\
\hline NGC 5624 & III & 0 & 46 & 50 & 87 & 48 & 7.3 & 9.9 & 1.5 & 0.5 & 20.1 & 22.6 & 24.9 & 0.05 \\
\hline & & 0 & 37 & 47 & 80 & 42 & 6.4 & 8.9 & 1.3 & 0.4 & 20.3 & 23.2 & 25.4 & 0.07 \\
\hline NGC 5660 & II-CT & 12 & 63 & 70 & 90 & $\begin{array}{l}67 \\
60\end{array}$ & 12.0 & 17.8 & 3.2 & 1.6 & 19.9 & 17.3 & 24.1 & 0.06 \\
\hline & & $\begin{array}{r}12 \\
*\end{array}$ & $\begin{array}{r}64 \\
*\end{array}$ & $\begin{array}{r}71 \\
*\end{array}$ & $\begin{array}{r}105 \\
*\end{array}$ & $\begin{array}{r}69 \\
*\end{array}$ & $\begin{array}{r}12.4 \\
*\end{array}$ & $\begin{array}{r}18.5 \\
*\end{array}$ & 3.3 & $\begin{array}{r}1.7 \\
*\end{array}$ & $\begin{array}{r}20.4 \\
*\end{array}$ & 17.8 & 24.2 & 0.08 \\
\hline NGC 5667 & III & 28 & 64 & 66 & 96 & 64 & 9.8 & 11.0 & 1.7 & 0.6 & 19.1 & 21.9 & 25.7 & 0.03 \\
\hline & & * & $*$ & * & $*$ & * & * & $*$ & $*$ & $*$ & * & $*$ & & 0.04 \\
\hline & & 28 & 62 & 65 & 111 & 63 & 9.7 & 10.3 & 1.6 & 0.4 & 19.2 & 23.7 & 26.4 & 0.04 \\
\hline NGC 5668 & I & 20 & 178 & $*$ & $*$ & $*$ & $*$ & 26.7 & 3.1 & $*$ & 20.6 & 0.0 & 20.6 & 0.10 \\
\hline & 1 & 20 & 162 & $*$ & $*$ & $*$ & $*$ & 24.3 & 2.8 & $*$ & 20.7 & 0.0 & 20.7 & 0.14 \\
\hline NGC 5693 & II-AB & $\begin{array}{l}9 \\
9\end{array}$ & $\begin{array}{l}28 \\
26\end{array}$ & $\begin{array}{l}50 \\
51\end{array}$ & $\begin{array}{l}71 \\
71\end{array}$ & $\begin{array}{l}29 \\
32\end{array}$ & $\begin{array}{l}5.1 \\
5.6\end{array}$ & $\begin{array}{l}16.7 \\
20.5\end{array}$ & $\begin{array}{l}2.9 \\
3.6\end{array}$ & $\begin{array}{l}2.1 \\
2.2\end{array}$ & $\begin{array}{l}20.9 \\
21.6\end{array}$ & $\begin{array}{l}17.9 \\
19.4\end{array}$ & $\begin{array}{l}23.7 \\
23.5\end{array}$ & $\begin{array}{l}0.10 \\
0.14\end{array}$ \\
\hline NGC 5713 & III & 8 & 102 & 114 & 164 & 103 & 14.0 & 18.1 & 2.5 & 0.7 & 19.1 & 21.0 & 25.6 & 0.11 \\
\hline & & 8 & $\begin{array}{r}112 \\
95\end{array}$ & * & $\begin{array}{l}9 \\
*\end{array}$ & * & * & 18.1 & 2.5 & $\begin{array}{l}.7 \\
*\end{array}$ & $\begin{array}{l}19.6 \\
199\end{array}$ & $\begin{array}{r}21.5 \\
0.0\end{array}$ & $\begin{array}{l}25.9 \\
109\end{array}$ & 0.15 \\
\hline NGC 5768 & 1 & 6 & 98 & * & * & * & * & 13.2 & $\begin{array}{l}1.0 \\
1.8\end{array}$ & * & 20.4 & 0.0 & 20.4 & 0.35 \\
\hline 1067 & ULo-OL R & $*$ & $*$ & 40 & 99 & * & $*$ & $*$ & $*$ & * & * & 19.8 & $*$ & 0.14 \\
\hline $00 /$ & 11.0-ULK & $*$ & $*$ & 40 & 80 & * & $*$ & * & 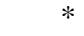 & * & * & 20.0 & * & 0.19 \\
\hline & & 43 & 81 & $\begin{array}{r}86 \\
118\end{array}$ & $\begin{array}{l}116 \\
182\end{array}$ & $\begin{array}{r}86 \\
116\end{array}$ & 9.9 & 50.2 & 5.8 & 2.3 & $\begin{array}{l}22.1 \\
199\end{array}$ & 19.9 & 23.9 & 0.12 \\
\hline NGC 5774 & II.o-OLR + III & $\begin{array}{l}86 \\
47\end{array}$ & $\begin{array}{r}116 \\
74\end{array}$ & $\begin{array}{r}118 \\
77\end{array}$ & $\begin{array}{l}182 \\
124\end{array}$ & $\begin{array}{r}116 \\
77\end{array}$ & $\begin{array}{r}13.3 \\
88\end{array}$ & $\begin{array}{l}22.2 \\
608\end{array}$ & $\begin{array}{l}2.5 \\
7.0\end{array}$ & $\begin{array}{l}0.1 \\
27\end{array}$ & $\begin{array}{l}19.9 \\
227\end{array}$ & & 25.1 & 0.12 \\
\hline & & $\begin{array}{l}47 \\
77\end{array}$ & $\begin{array}{r}74 \\
124\end{array}$ & $\begin{array}{r}77 \\
125\end{array}$ & $\begin{array}{l}124 \\
182\end{array}$ & $\begin{array}{r}77 \\
124\end{array}$ & $\begin{array}{r}8.8 \\
14.2\end{array}$ & $\begin{array}{l}60.8 \\
22.9\end{array}$ & $\begin{array}{l}7.0 \\
2.6\end{array}$ & $\begin{array}{l}2.7 \\
0.6\end{array}$ & $\begin{array}{l}22.7 \\
20.5\end{array}$ & $\begin{array}{l}20.5 \\
22.3\end{array}$ & $\begin{array}{l}24.1 \\
25.7\end{array}$ & $\begin{array}{l}0.16 \\
0.16\end{array}$ \\
\hline NGC 5806 & III & $\begin{array}{l}60 \\
60\end{array}$ & $\begin{array}{l}116 \\
123\end{array}$ & $\begin{array}{l}118 \\
124\end{array}$ & $\begin{array}{l}242 \\
257\end{array}$ & $\begin{array}{l}118 \\
124\end{array}$ & $\begin{array}{l}11.8 \\
12.4\end{array}$ & $\begin{array}{l}29.9 \\
31.4\end{array}$ & $\begin{array}{l}3.0 \\
3.1\end{array}$ & $\begin{array}{l}0.5 \\
0.5\end{array}$ & $\begin{array}{l}20.3 \\
21.1\end{array}$ & $\begin{array}{l}22.3 \\
23.0\end{array}$ & $\begin{array}{l}24.6 \\
25.3\end{array}$ & $\begin{array}{l}0.14 \\
0.20\end{array}$ \\
\hline NGC 5850 & II.o-OLR & 96 & 136 & 140 & 233 & 137 & 25.0 & 92.2 & 16.8 & 2.7 & 22.3 & 19.7 & 23.8 & 0.16 \\
\hline (N) & 11.0-ULN & 96 & 133 & 138 & 240 & 138 & 25.2 & 127.1 & 23.2 & 3.9 & 23.3 & 20.0 & 24.4 & 0.21 \\
\hline
\end{tabular}


Table 3. continued.

\begin{tabular}{|c|c|c|c|c|c|c|c|c|c|c|c|c|c|c|}
\hline \multirow{3}{*}{$\begin{array}{l}\text { Galaxy } \\
\text { (1) }\end{array}$} & \multirow{3}{*}{$\begin{array}{l}\text { Break } \\
\text { type } \\
(2)\end{array}$} & \multirow{3}{*}{$\begin{array}{c}b_{1} \\
{\left[{ }^{\prime \prime}\right]}\end{array}$} & \multirow{3}{*}{$\begin{array}{c}b_{2} \\
{\left[{ }^{\prime \prime}\right]}\end{array}$} & \multirow{2}{*}{$\begin{array}{c}b_{3} \\
{\left[{ }^{\prime \prime}\right]}\end{array}$} & \multirow{3}{*}{$\begin{array}{c}b_{4} \\
{\left[{ }^{\prime \prime}\right]}\end{array}$} & \multicolumn{2}{|c|}{$R_{\mathrm{br}}$} & \multicolumn{2}{|c|}{$h_{\text {in }}$} & \multirow{3}{*}{$\begin{array}{c}h_{\text {in }} \\
{\left[\begin{array}{l}\left.h_{\text {out }}\right] \\
\quad(6)\end{array}\right.}\end{array}$} & \multirow{3}{*}{$\begin{array}{c}\mu_{0, \text { in }} \\
{\left[\frac{\mathrm{mag}}{\mathrm{a}^{\prime \prime}}\right]}\end{array}$} & \multirow{3}{*}{$\begin{array}{l}\mu_{0, \text { out }} \\
{\left[\frac{\mathrm{mag}}{\mathrm{口}^{\prime \prime}}\right]}\end{array}$} & \multirow{3}{*}{$\begin{array}{c}\mu_{\mathrm{br}} \\
{\left[\frac{\mathrm{mag}}{\square^{\prime \prime}}\right]} \\
(8)\end{array}$} & \multirow{3}{*}{$\begin{array}{c}A_{r^{\prime} / g^{\prime}} \\
{[\mathrm{mag}]} \\
\quad(9)\end{array}$} \\
\hline & & & & & & {$\left[{ }^{\prime \prime}\right]$} & {$[\mathrm{kpc}]$} & {$\left[{ }^{\prime \prime}\right]$} & {$[\mathrm{kpc}]$} & & & & & \\
\hline & & & & (3) & & & 4) & & & & & & & \\
\hline UGC 09741 & III & $\begin{array}{l}17 \\
17\end{array}$ & $\begin{array}{l}24 \\
27\end{array}$ & $\begin{array}{l}29 \\
29\end{array}$ & $\begin{array}{l}62 \\
62\end{array}$ & $\begin{array}{l}25 \\
27\end{array}$ & $\begin{array}{l}4.7 \\
5.1\end{array}$ & $\begin{array}{l}6.8 \\
6.9\end{array}$ & $\begin{array}{l}1.3 \\
1.3\end{array}$ & $\begin{array}{l}0.6 \\
0.7\end{array}$ & $\begin{array}{l}19.8 \\
20.4\end{array}$ & $\begin{array}{l}21.2 \\
21.6\end{array}$ & $\begin{array}{l}23.5 \\
24.0\end{array}$ & $\begin{array}{l}0.06 \\
0.09\end{array}$ \\
\hline UGC 09837 & II-CT & $\begin{array}{l}8 \\
8\end{array}$ & $\begin{array}{l}45 \\
44\end{array}$ & $\begin{array}{l}53 \\
46\end{array}$ & $\begin{array}{l}69 \\
69\end{array}$ & $\begin{array}{l}46 \\
46\end{array}$ & $\begin{array}{l}9.4 \\
9.4\end{array}$ & $\begin{array}{l}17.1 \\
18.2\end{array}$ & $\begin{array}{l}3.5 \\
3.7\end{array}$ & $\begin{array}{l}2.4 \\
2.6\end{array}$ & $\begin{array}{l}21.4 \\
21.8\end{array}$ & $\begin{array}{l}16.6 \\
17.0\end{array}$ & $\begin{array}{l}24.6 \\
24.8\end{array}$ & $\begin{array}{l}0.05 \\
0.06\end{array}$ \\
\hline NGC 5937 & III & $\begin{array}{l}12 \\
12\end{array}$ & $\begin{array}{l}62 \\
68\end{array}$ & $\begin{array}{l}75 \\
70\end{array}$ & $\begin{array}{r}117 \\
98\end{array}$ & $\begin{array}{l}63 \\
68\end{array}$ & $\begin{array}{l}12.5 \\
13.5\end{array}$ & $\begin{array}{l}12.6 \\
12.8\end{array}$ & $\begin{array}{l}2.5 \\
2.5\end{array}$ & $\begin{array}{l}0.6 \\
0.6\end{array}$ & $\begin{array}{l}19.1 \\
19.8\end{array}$ & $\begin{array}{l}21.1 \\
22.2\end{array}$ & $\begin{array}{l}24.6 \\
25.8\end{array}$ & $\begin{array}{l}0.50 \\
0.69\end{array}$ \\
\hline IC 1125 & I & $\begin{array}{l}4 \\
4\end{array}$ & $\begin{array}{l}77 \\
68\end{array}$ & $\begin{array}{l}* \\
*\end{array}$ & $\begin{array}{l}* \\
*\end{array}$ & $\begin{array}{l}* \\
*\end{array}$ & $\begin{array}{l}* \\
*\end{array}$ & $\begin{array}{l}11.4 \\
11.6\end{array}$ & $\begin{array}{l}2.3 \\
2.3\end{array}$ & $\begin{array}{l}* \\
*\end{array}$ & $\begin{array}{l}19.9 \\
20.5\end{array}$ & $\begin{array}{l}0.0 \\
0.0\end{array}$ & $\begin{array}{l}19.9 \\
20.5\end{array}$ & $\begin{array}{l}0.43 \\
0.59\end{array}$ \\
\hline IC 1158 & II.o-CT & $\begin{array}{l}14 \\
14\end{array}$ & $\begin{array}{l}53 \\
50\end{array}$ & $\begin{array}{l}67 \\
67\end{array}$ & $\begin{array}{r}98 \\
101\end{array}$ & $\begin{array}{l}65 \\
61\end{array}$ & $\begin{array}{l}9.1 \\
8.5\end{array}$ & $\begin{array}{l}30.5 \\
34.8\end{array}$ & $\begin{array}{l}4.3 \\
4.9\end{array}$ & $\begin{array}{l}2.9 \\
3.3\end{array}$ & $\begin{array}{l}21.2 \\
21.9\end{array}$ & $\begin{array}{l}16.9 \\
17.5\end{array}$ & $\begin{array}{l}23.4 \\
23.8\end{array}$ & $\begin{array}{l}0.32 \\
0.44\end{array}$ \\
\hline NGC 6070 & II.o-CT & $\begin{array}{l}20 \\
20\end{array}$ & $\begin{array}{l}87 \\
87\end{array}$ & $\begin{array}{l}108 \\
108\end{array}$ & $\begin{array}{l}162 \\
157\end{array}$ & $\begin{array}{l}97 \\
88\end{array}$ & $\begin{array}{l}14.0 \\
12.7\end{array}$ & $\begin{array}{l}37.2 \\
43.4\end{array}$ & $\begin{array}{l}5.4 \\
6.3\end{array}$ & $\begin{array}{l}2.0 \\
2.6\end{array}$ & $\begin{array}{l}20.1 \\
21.0\end{array}$ & $\begin{array}{l}17.3 \\
17.4\end{array}$ & $\begin{array}{l}22.8 \\
23.3\end{array}$ & $\begin{array}{l}0.41 \\
0.56\end{array}$ \\
\hline NGC 6155 & II-AB & $\begin{array}{l}4 \\
4\end{array}$ & $\begin{array}{l}33 \\
33\end{array}$ & $\begin{array}{l}36 \\
37\end{array}$ & $\begin{array}{l}71 \\
63\end{array}$ & $\begin{array}{l}34 \\
34\end{array}$ & $\begin{array}{l}6.3 \\
6.3\end{array}$ & $\begin{array}{l}12.2 \\
13.1\end{array}$ & $\begin{array}{l}2.3 \\
2.4\end{array}$ & $\begin{array}{l}1.5 \\
1.8\end{array}$ & $\begin{array}{l}19.4 \\
20.0\end{array}$ & $\begin{array}{l}18.2 \\
17.9\end{array}$ & $\begin{array}{l}21.9 \\
22.7\end{array}$ & $\begin{array}{l}0.04 \\
0.05\end{array}$ \\
\hline UGC 10721 & III & $\begin{array}{l}16 \\
16\end{array}$ & $\begin{array}{l}38 \\
38\end{array}$ & $\begin{array}{l}48 \\
46\end{array}$ & $\begin{array}{l}80 \\
78\end{array}$ & $\begin{array}{l}41 \\
41\end{array}$ & $\begin{array}{l}8.9 \\
8.9\end{array}$ & $\begin{array}{l}8.3 \\
7.9\end{array}$ & $\begin{array}{l}1.8 \\
1.7\end{array}$ & $\begin{array}{l}0.5 \\
0.6\end{array}$ & $\begin{array}{l}19.3 \\
19.5\end{array}$ & $\begin{array}{l}21.7 \\
21.8\end{array}$ & $\begin{array}{l}24.6 \\
24.8\end{array}$ & $\begin{array}{l}0.10 \\
0.13\end{array}$ \\
\hline NGC 7437 & II-CT & $\begin{array}{l}12 \\
12\end{array}$ & $\begin{array}{l}39 \\
40\end{array}$ & $\begin{array}{l}47 \\
43\end{array}$ & $\begin{array}{r}91 \\
147\end{array}$ & $\begin{array}{l}43 \\
41\end{array}$ & $\begin{array}{l}6.5 \\
6.2\end{array}$ & $\begin{array}{l}26.4 \\
27.1\end{array}$ & $\begin{array}{l}4.0 \\
4.1\end{array}$ & $\begin{array}{l}1.9 \\
1.9\end{array}$ & $\begin{array}{l}21.3 \\
21.8\end{array}$ & $\begin{array}{l}19.7 \\
20.4\end{array}$ & $\begin{array}{l}23.1 \\
23.3\end{array}$ & $\begin{array}{l}0.11 \\
0.15\end{array}$ \\
\hline NGC 7606 & II-CT & $\begin{array}{l}20 \\
20\end{array}$ & $\begin{array}{l}96 \\
92\end{array}$ & $\begin{array}{l}113 \\
114\end{array}$ & $\begin{array}{l}195 \\
187\end{array}$ & $\begin{array}{l}97 \\
93\end{array}$ & $\begin{array}{l}14.7 \\
14.1\end{array}$ & $\begin{array}{l}43.4 \\
49.9\end{array}$ & $\begin{array}{l}6.6 \\
7.6\end{array}$ & $\begin{array}{l}1.9 \\
2.2\end{array}$ & $\begin{array}{l}19.6 \\
20.5\end{array}$ & $\begin{array}{l}17.3 \\
17.7\end{array}$ & $\begin{array}{l}22.3 \\
22.9\end{array}$ & $\begin{array}{l}0.10 \\
0.14\end{array}$ \\
\hline UGC 12709 & II-CT & $\begin{array}{l}5 \\
5\end{array}$ & $\begin{array}{l}65 \\
64\end{array}$ & $\begin{array}{l}72 \\
66\end{array}$ & $\begin{array}{l}102 \\
108\end{array}$ & $\begin{array}{l}69 \\
66\end{array}$ & $\begin{array}{l}12.8 \\
12.2\end{array}$ & $\begin{array}{l}29.8 \\
33.0\end{array}$ & $\begin{array}{l}5.5 \\
6.1\end{array}$ & $\begin{array}{l}2.2 \\
2.4\end{array}$ & $\begin{array}{l}22.4 \\
23.0\end{array}$ & $\begin{array}{l}19.3 \\
19.8\end{array}$ & $\begin{array}{l}25.1 \\
25.2\end{array}$ & $\begin{array}{l}0.11 \\
0.15\end{array}$ \\
\hline
\end{tabular}




\section{Appendix A: Galaxy atlas}

The atlas presented here contain the 85 (from 98) galaxies with useful images in the SDSS Second Data Release (DR2) as described in Sect. 2.1. The data for every two galaxies is presented on a single page. On the left side we give detailed comments on the individual galaxies concerning the applied classification and global characteristics (such as distinct morphological features or environment). The headline gives the name and classification type. Below we reproduce the main parameters: Position (J2000), RC3 Hubble-type, coded LEDA Hubble parameter T, absolute $B$ band magnitude $\mathrm{M}$ abs [B-mag], apparent diameter ['], virgocentric radial velocity vvir $\left[\mathrm{km} \mathrm{s}^{-1}\right]$. On the right side we reproduce the JPEG $800 \times 600$ colour mosaics obtained with the SDSS "Finding Chart" tool ${ }^{6}$. Overlayed are a N-S, E-W grid through the center, a $1^{\prime}$ scale bar, and the outline of the individual SDSS fields. In addition we show azimuthally averaged, radial surface brightness profiles in the $g^{\prime}$ (triangles) and $r^{\prime}$ (circles) band obtained from fixed ellipse fits. The $r^{\prime}$-band is overlayed by the best exponential fits (cf. Sect. 4.2) to the individual regions: single disk, inner and outer disk, or three fits in the case of a mixed classification. The boundaries $\left(b_{1-4}\right)$ used for the fit are marked with the short vertical, dashed lines on the abscissa. Following Courteau (1996) we also chose not to show error bars on all the profiles to avoid overcrowding of the plots. Instead, we show for the $g^{\prime}$-band (horizontal, dashed line) and for the $r^{\prime}$-band (horizontal, dotted line) the critical surface brightness $\left(\mu_{\text {crit }}\right)$ down to which the profile is reliable. This limit is placed where profiles obtained by using $\pm 1 \sigma s k y$ deviate by more than 0.2 mag from the one with our mean sky value (cf. Sect. 3.3). In the upper right corner of the surface brightness profiles we note the galaxy name, the Hubble type according to RC3 (for galaxies without or uncertain (S?) classification from LEDA), and the profile type.

${ }^{6}$ http://cas.sdss.org/dr4/en/tools/chart/chart.asp 
IC 1067: Type II.o-OLR (possible Type I)

J145305.2+031954.SBS3. $3.0-18.972 .01665$

Galaxy close to the border of the SDSS field but almost complete with a nearby, physical companion (IC 1066, $v=$ $1577 \mathrm{~km} \mathrm{~s}^{-1}$ ) only $2.2^{\prime}$ away, which slightly influences the background estimation (together with two nearby bright stars). The strong peak at $\sim 20^{\prime \prime}$ in the final profile is related to the ring around the prominent bar and the dip at $\sim 35^{\prime \prime}$ to the inter-arm region of the two symmetric spiral arms peaking at $\sim 40^{\prime \prime}$. Although possible to fit (at least for the $r^{\prime}$ band profile) with a single exponential (Type I), we classify the galaxy as Type II.o-OLR, with a break around twice the bar radius at $\sim 40^{\prime \prime}$, having an inner disk region too small to be detected with our automatic break searching routine. This is consistent with the classification and profile by Erwin et al. (2006). The region inside $\sim 40^{\prime \prime}$ is excluded for the fit of the outer scalelength.

IC 1125: Type I

J153305.6-013742 .S. .8* $7.7-20.051 .72868$

According to the coordinates in NED the companion (KPG 467A) in Karachentsev's Isolated Pairs of Galaxies Catalogue Karachentsev (1972) corresponds only to a faint starlike object about $11^{\prime \prime}$ away. The small bump in the radial profile at $\sim 40^{\prime \prime}$ corresponds to an outer spiral arms. Otherwise we see no obvious deviation from a single exponential and therefore the galaxy is classified as Type I.
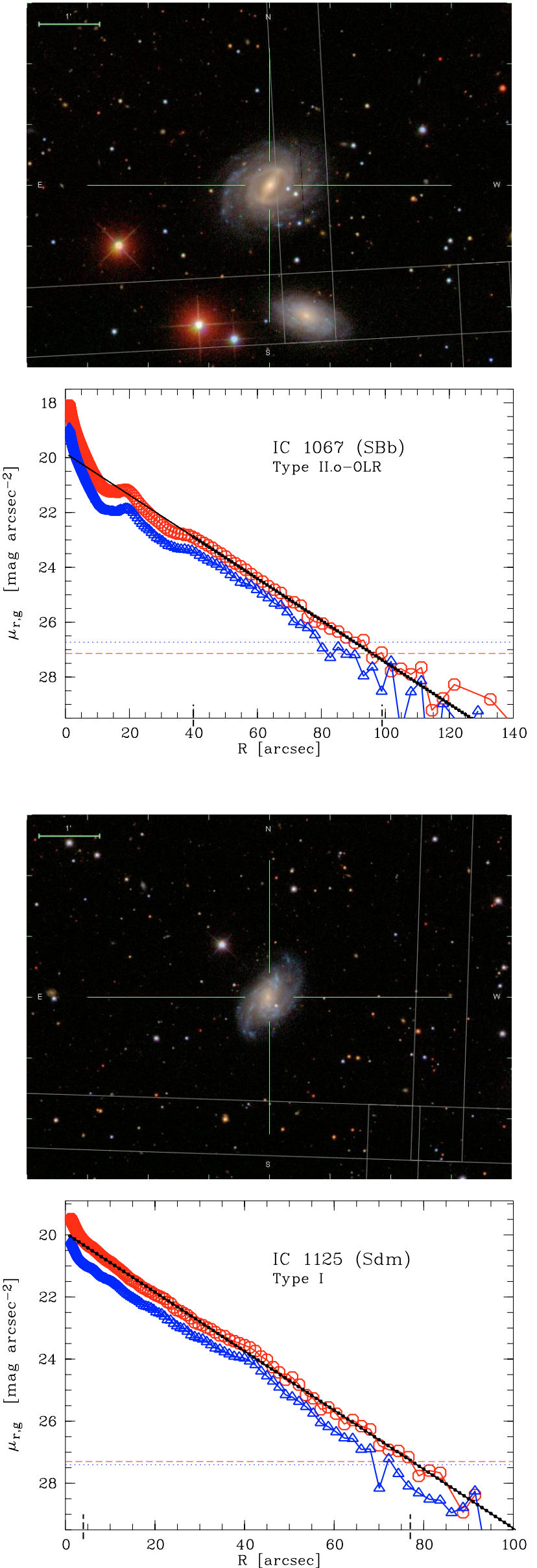
IC 1158: Type II.o-CT

J160134.1+014228.SXR5*5.3 -19.36 2.22018

Galaxy shows clearly a truncated profile with an extended break region, starting approximately at the end of the spiral arms. The center looks like a secondary bar with a small ring embedded in larger bar of size roughly $\$ 22^{\prime \prime}$ (without any noticeable feature in the final profile). So the break with downbending profile at $\sim 60^{\prime \prime}$ is well beyond a typical Type II.oOLR break.

\section{NGC 0450: Type II.o-CT}

J011530.8-005138 .SXS6*6.0 $6-19.633 .01712$

A background galaxy (UGC 00807, $11587 \mathrm{~km} \mathrm{~s}^{-1}$ ) is superimposed. The mean ellipticity of the outer disk is difficult to determine. The inner disk looks fairly round whereas the outer disk looks rather elliptical. The size of the slightly more elliptical inner part, associated to be the bar (although without any noticeable feature in the final profile), is roughly $\sim 8^{\prime \prime}$, so the break with the downbending profile at $\sim 84^{\prime \prime}$ is well beyond a typical Type II.o-OLR break and roughly outside the spiral arm region.
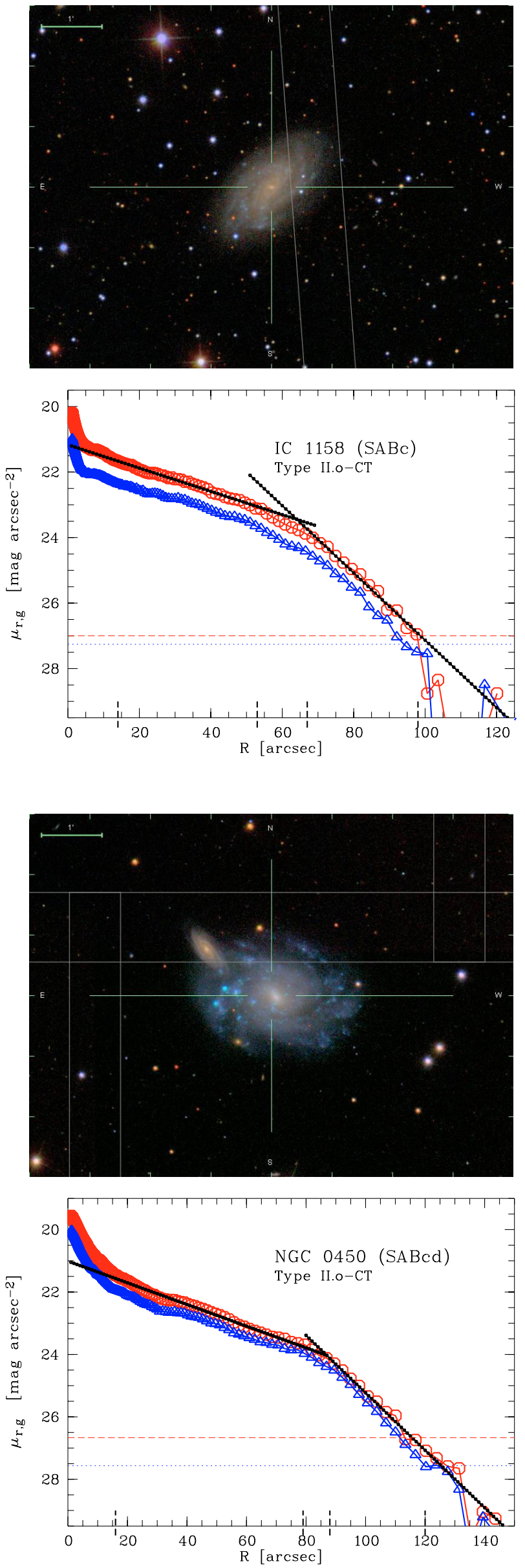
NGC 0701: Type II.i (possible Type I)

J015103.8-094209 .SBT5. $5.1-19.742 .51729$

Galaxy is in a multiple system together with NGC 0681 and NGC 4594 (where PGC 006667 (see below) is also part of) with an additional small physical companion (IC 1738, $v=$ $1750 \mathrm{~km} \mathrm{~s}^{-1}$ ) about $6^{\prime}$ away. Galaxy close to our high axis ratio limit so the dust may have some influence. The inner region shows no nucleus, so the centering is done from the outer isophotes. The extend of the bar is unknown, but the visible bump at $45^{\prime \prime}$ is probably not related to a bar. It is possible to construct a break at 45" (which would be more pronounced in the $g^{\prime}$ band), but the inner drop could be also due to the higher inclination with dust and/or patchy star formation affecting the profile.

NGC 0853: Type III

J021141.5-091817 .S..9P $8.3-18.42 \quad 1.51405$

The inner region shows no clear nucleus but many $\mathrm{H}$ II regions, so the centering is done from the outer isophotes. The inner disk is slightly asymmetric with an additional light patch in the western part, therefore mean ellipticity and PA difficult to fix. Almost continuously upbending profile beyond the peak at 15", which includes all H II regions, getting flat at $\sim 42^{\prime \prime}$. The inner profile is rather curved and not well approximated with a single exponential. The apparent outer break at $\sim 100^{\prime \prime}$ is only due to the improper fixed ellipse fit in the outer parts.
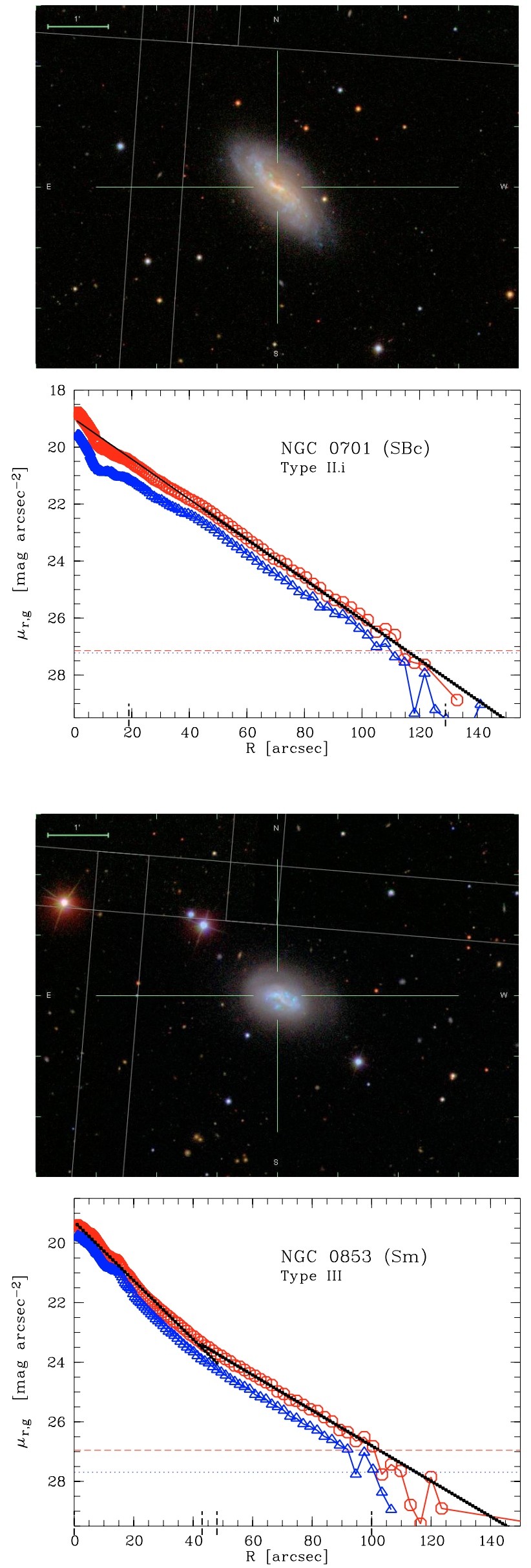
NGC 0941: Type II-CT

J022827.9-010906.SXT5. $5.4-19.02 \quad 2.51535$

Galaxy shows only a very small, point-like nucleus and is in a small group of galaxies (including NGC 936 and NGC 955). The small dip at $\sim 40^{\prime \prime}$ is associated with the inner spiral arm region. Although classified as SAB no real bar visible on image or in the profile thus the downbending break is classified as Type II-CT.
NGC 1042: Type II-AB

J024023.9-082558.SXT6. $6.1-19.834 .41264$

A large scale gradient (from top to bottom) in the background of the $r^{\prime}$ and $g^{\prime}$ band image is removed with a linear fit. Only partly fitted since $\lesssim 1 / 3$ of galaxy is beyond SDSS field. Galaxy shows an asymmetric (frayed) extension towards the south-east and a sharper edge towards the north-west, which makes it not really lopsided but looking rather like being affected by moving in a dense IGM. The extended (frayed) spiral arms build the outer disk, so ellipticity and PA very difficult to fix. The inner bump in the profile at $\sim 90^{\prime \prime}$ is due to spiral arms. The inner profile is below the inward extrapolation of the outer disk, but due to the asymmetric shape of the disk we call this galaxy only Type II-AB.
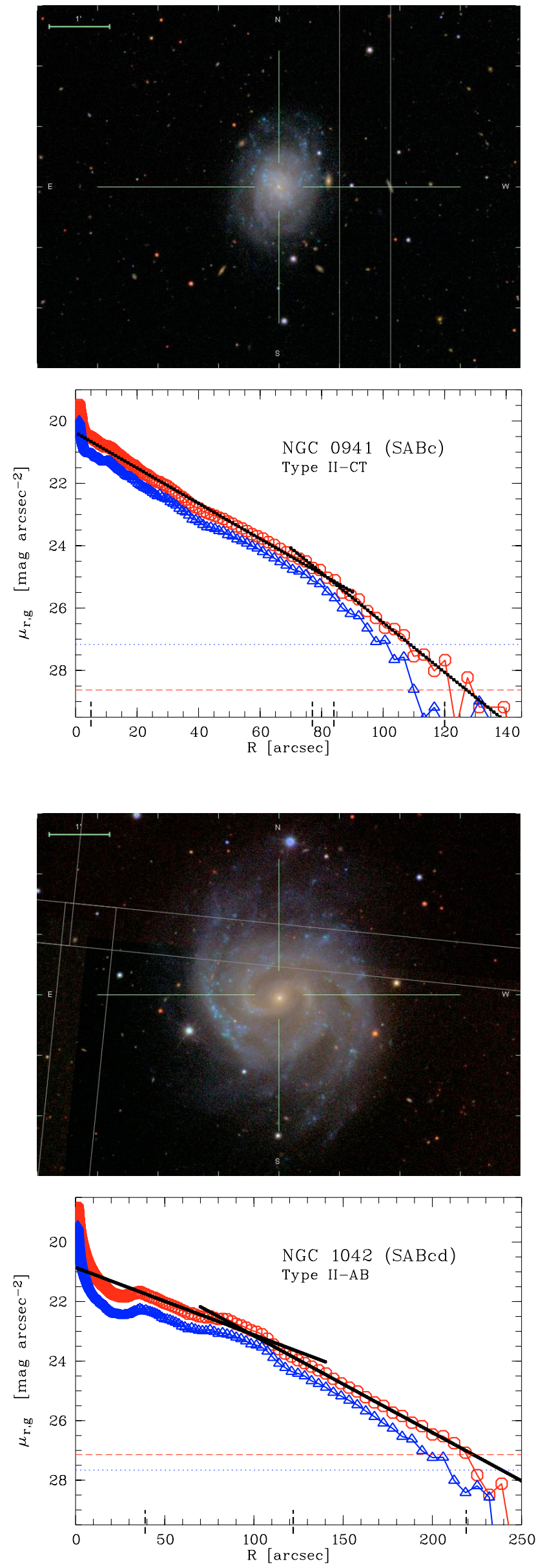
NGC 1068 = M 77: Type II.o-OLR

J024240.8-000048 RSAT3. $3.0-21.697 .61068$

Only partly fitted since $\lesssim 1 / 3$ of galaxy is beyond the SDSS field. Galaxy appears to have an inner disk, elongated into a bar-like structure sitting in an outer disk with clearly different PA and with indication for an additional outer ring at 200". According to Erwin (2004) it is a double-barred galaxy with an inner bar of size $\sim 15-20^{\prime \prime}$ and an outer bar which is very oval (not very strong) and very large (extending out to $\lesssim 100^{\prime \prime}$; as seen by e.g. Schinnerer et al. 2000). The galaxy looks in this sense similar to NGC 5248 (Jogee et al. 2002). This peculiar shape makes its very difficult to decide on a mean ellipticity and PA of the outer disk from the photometry. The position of the outer ring corresponds to the downbending break at $\sim 190 "$ and is therefore roughly twice the bar radius. This suggests the Type II.o-OLR classification, although the profile looks at first glance more like a Type III with a break at $\sim 100 "$.

NGC 1084: Type III

J024559.7-073437 .SAS5. $5.1 \quad-20.203 .21299$

A large scale gradient (from top to bottom) in the background of the $r^{\prime}$ band image is removed with a linear fit. Slightly inclined galaxy sitting clearly in a more roundish outer envelope, beyond which some stream-like remnants of a possible recent interaction are visible. Small edge-on galaxy along the stream path is confirmed to be only a background galaxy. According to SDSS spectroscopy there is another object inside the disk (RA: 024600.3, Dec -073417) with similar velocity and associated to a visible light concentration. The peculiar shape of the outer disk region makes it very difficult to decide on a mean ellipticity and PA from the photometry. The resulting profile inside the break at $\sim 90^{\prime \prime}$ is rather curved towards the center with additional kinks $\left(\sim 25^{\prime \prime}\right.$ and $\left.\sim 50^{\prime \prime}\right)$, so it is not clear what the galaxy might be inside the Type III envelope.
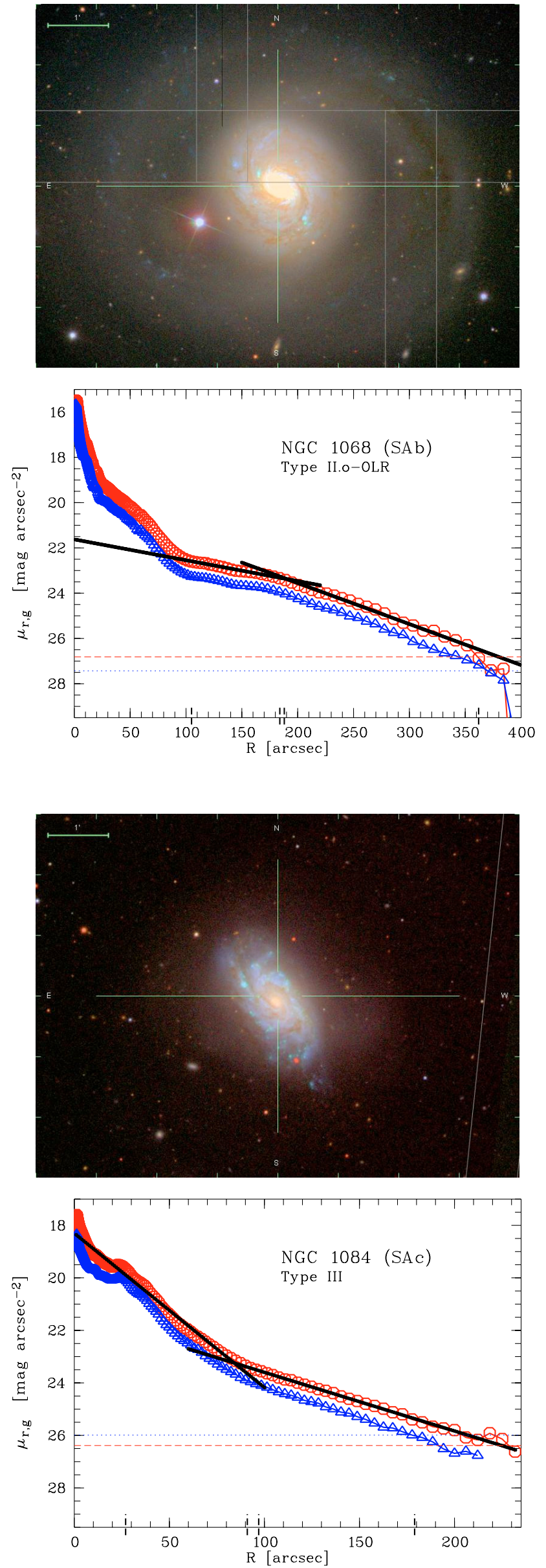
NGC 1087: Type III

J024625.2-002955.SXT5. $5.3-20.46 \quad 3.7 \quad 1443$

An apparent faint low surface brightness (center-less) structure towards the North is only scattered light from a nearby star. The galaxy center is not well defined, it shows only a small bar-like structure with a double nucleus, so we used the slightly lopsided (but symmetric) outer disk for centering, which accounts for the central drop inside $\sim 50 "$ in the final profile. There is no spiral structure visible in the outer disk. The transition seems to be rather sharp, but the inner profile is almost curved, so it is not clear what its break type is inside the Type III envelope.

NGC 1299: Type III

J032009.4-061550.SBT3? $3.2 \quad-19.25 \quad 1.22197$

Galaxy is small and close to our high axis ratio limit. The background exhibits a gradient but the galaxy is small enough to avoid fitting it. SDSS spectroscopy detects an object $0.2^{\prime}$ away from center with similar velocity. The rather symmetric outer disk shows no spiral structure but has slightly different ellipticity and PA compared to the inner part. The shoulder in the profile at $\sim 15^{\prime \prime}$ is related to the inner (bar-like) region with changing PA.
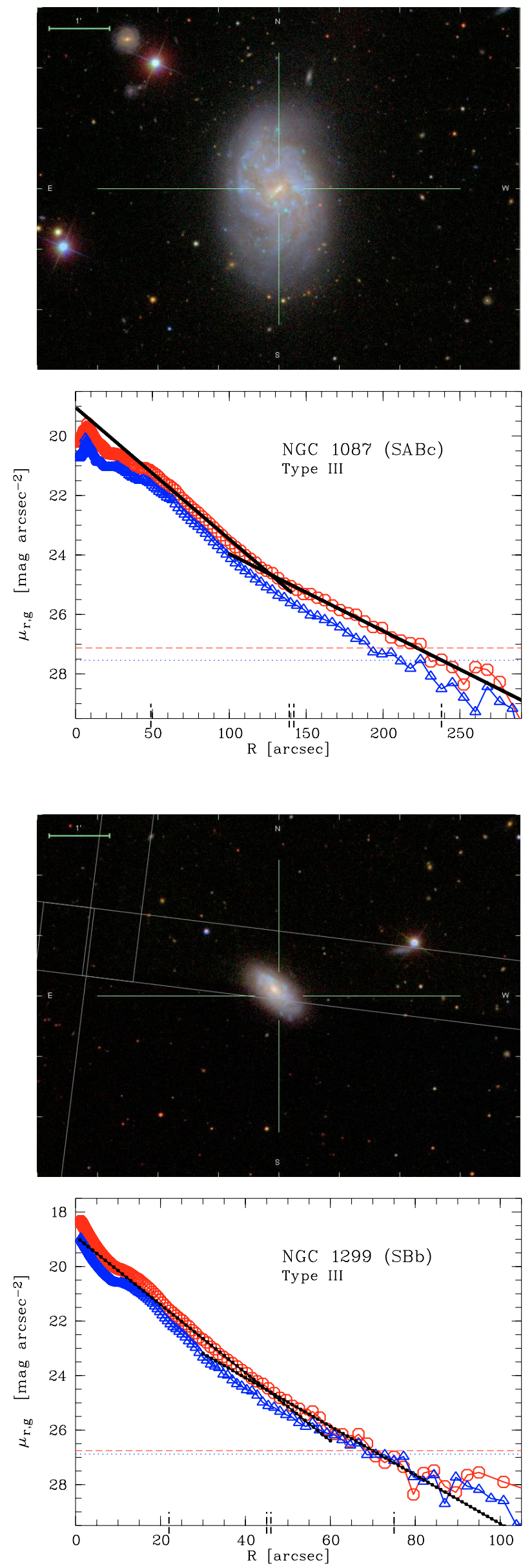
NGC 2541: Type II-CT

J081440.1+490341.SAS6. $6.2 \quad-18.505 .8734$

Galaxy with low surface brightness disk, having many patchy SF knots and an asymmetric (not well described with an ellipse), lopsided outer disk, which makes the chosen ellipticity and PA of the disk uncertain. The central peak is off by $\sim 8^{\prime \prime}$ compared to the outer disk where the centering is done, which causes the dip in the final profile close to the center. The downbending break at $\sim 140^{\prime \prime}$ is still inside the nearly symmetric part of the disk, thus classified as Type II-CT but this should be taken with some caution. This galaxy was classified as SB in our original LEDA catalogue but has been recently reclassified being now SAB. However, NED lists this galaxy as SA, using the RC3 classification, which is consistent with no bar being visible on the image or in the profile, so the downbending break is not a Type II.o-OLR break.

NGC 2543: Type II.i

J081258.0+361516 .SBS3. $3.1-20.662 .52590$

According to NED there is a dwarf elliptical galaxy $($ KUG 0809+363) at a similar distance. On the SDSS image (confirmed with DSS) there is an additional, low surface brightness structure visible at RA081308.5 and $\mathrm{DEC}+361635$, which is possibly another dwarf companion with an unknown distance. The galaxy is clearly double barred with a $R \sim 9^{\prime \prime}$ long secondary bar. Two bright, s-shaped spiral arms dominate the inner disk. The extended spiral arm towards the north-east is responsible for a slightly asymmetric outer disk. This structure makes the photometric measurement of the inclination (ellipticity) and PA very difficult. The bar and pseudo-ring build by the inner spiral arms are responsible for the prominent bump at $\sim 35^{\prime \prime}$ in the profile, followed by a dip inside, which leads to the Type II.i classification.
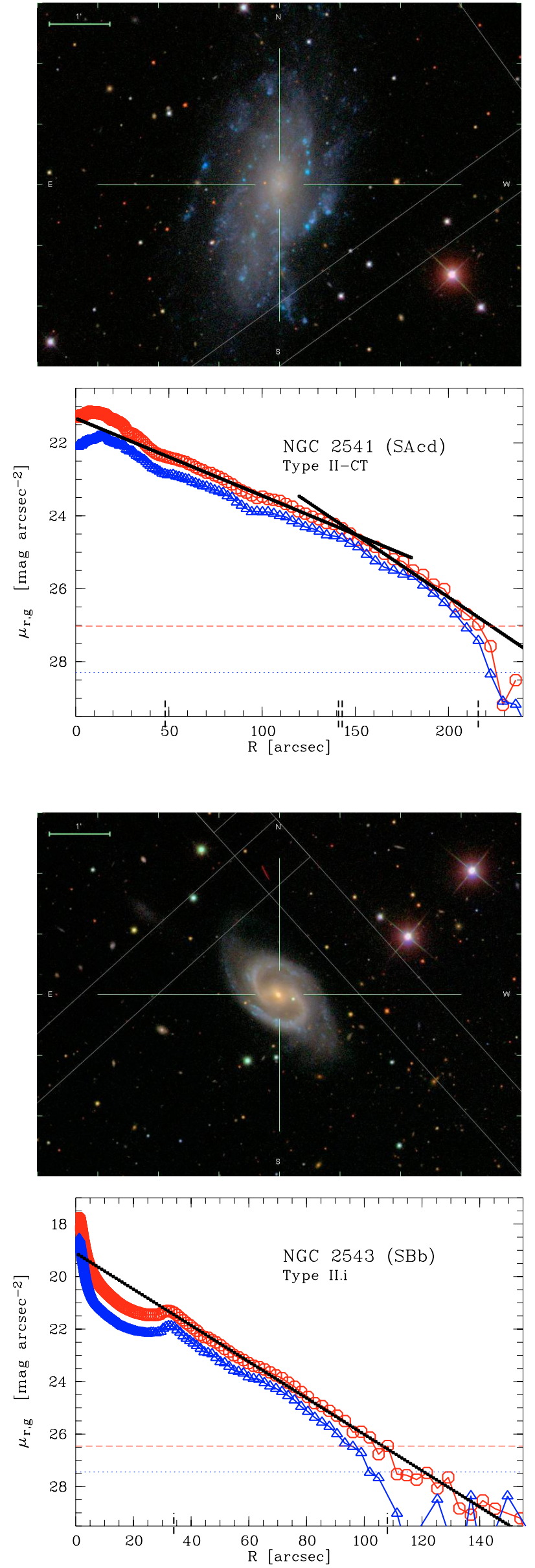
NGC 2684: Type II-CT

J085454.1+490937 .S?... $7.8-19.76 \quad 1.03043$

The rich cluster in background, with significantly redder colour, forces a large background mask covering a small part of the galaxy. Slightly asymmetric disk with bright center and no coherent spiral arms makes ellipticity and PA uncertain. Bump at $\sim 12^{\prime \prime}$ in profile is due to an inner ring-like structure, without an associated obvious bar structure. The break at $\sim 25^{\prime \prime}$ is not related to a morphological feature in the disk and, although at about twice the ring radius, classified as Type IICT. The upbending profile starting at $\sim 42^{\prime \prime}$ is most probably due to an improper sky subtraction caused by extended light from the background cluster.

NGC 2701: Type II-AB + III

J085905.9+534616.SXT5*5.1 -20.362 .12528$

According to NED there is a small companion (with similar velocity) superimposed on the galaxy disk towards the South at the end of a spiral arm which is masked here in addition to the bright star on the disk towards the West. The galaxy has a lopsided disk with the bar being offcenterd compared to the outer isophotes, where the centering is done. This is responsible for the dip in the center an possibly for the break at $\sim 42 "$, which is therefore called Type II-AB. The region beyond the second break at $\sim 70^{\prime \prime}$ corresponds to some faint, almost symmetric light around the galaxy with some large extension towards north-east, adding the classification Type III. This is possibly triggered by interaction with the dwarf companion at the other side.
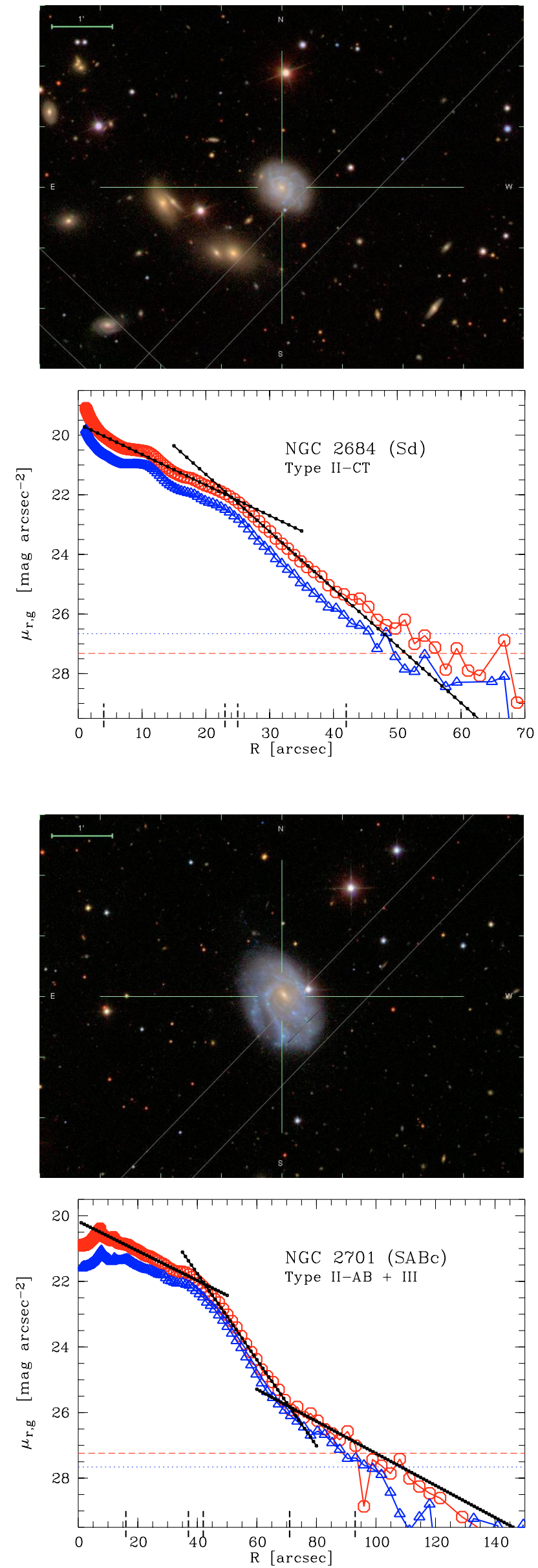
NGC 2776: Type I

J091214.3+445717 .SXT5. $5.1 \quad-21.04 \quad 2.92796$

The galaxy is classified as SAB but a bar is not obvious on the image. The background estimate is uncertain due to a nearby (off-field), extremely bright star. For the final profile we used a round ellipticity instead of the usual value at $1 \sigma$ (cf. Sect. 3.5) which gives an almost prototypical Type I profile with some minor wiggles, a bump at $\sim 25^{\prime \prime}$ due to the inner spiral arms, and some outer bumps due to some very weak spiral arm structures in the outer disk.
NGC 2967: Type III-d

J094203.3+002011 .SAS5. $4.8-20.262 .61858$

The galaxy is close to the edge of the SDSS field in an area with an increased background which is masked. Very faint, extended, but symmetric spiral arms inside the outer disk at $\sim 100^{\prime \prime}$ are visible, which do not continue inwards but start beyond the bump in the transition region at $\sim 75^{\prime \prime}$. The exact break radius is therefore uncertain.
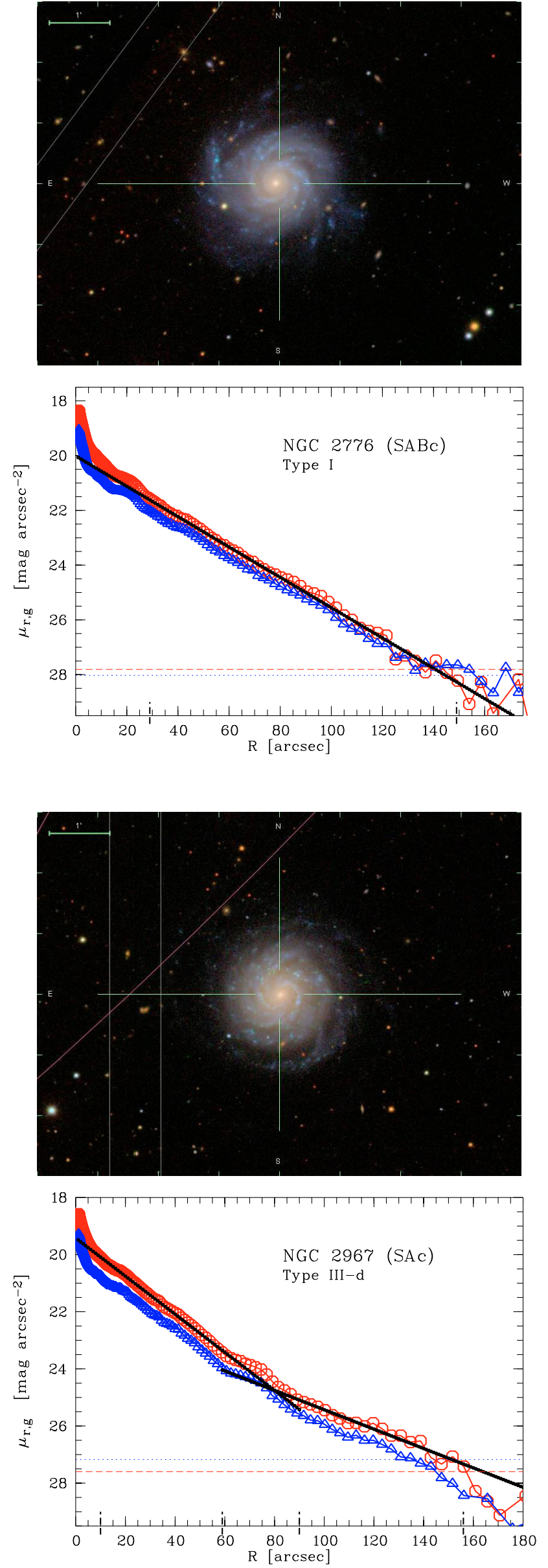
NGC 3055: Type II.o-CT

J095517.9+041611 .SXS5. $5.0-19.902 .11816$

Galaxy is classified as SAB and shows a narrow, thin bar, with the spiral arm structure wrapped around, which is responsible for the bump in the final profile at $\sim 18^{\prime \prime}$. The break with a downbending profile at $\sim 55^{\prime \prime}$ is well beyond a typical Type II.o-OLR break.

NGC 3246: Type II-AB

J102641.8+035143 .SX.8. 8.0 -18.91 2.2 2138

Galaxy is selected from the NGC catalogue Tully (1988) by (Pisano \& Wilcots 1999) as being isolated. Their H I measurements show a lopsided disk which resembles the one on the present SDSS image. The central bar region is clearly offcenterd compared to the outer isophote, which is used for the centering. The downbending break in the final profile and the downbending at the very center is therefore most probably only an apparent break due to the fixed ellipse fitting. Thus the galaxy is classified as Type II-AB. The background is rather disturbed by a bright star, but the galaxy is small enough not to be significantly influenced.
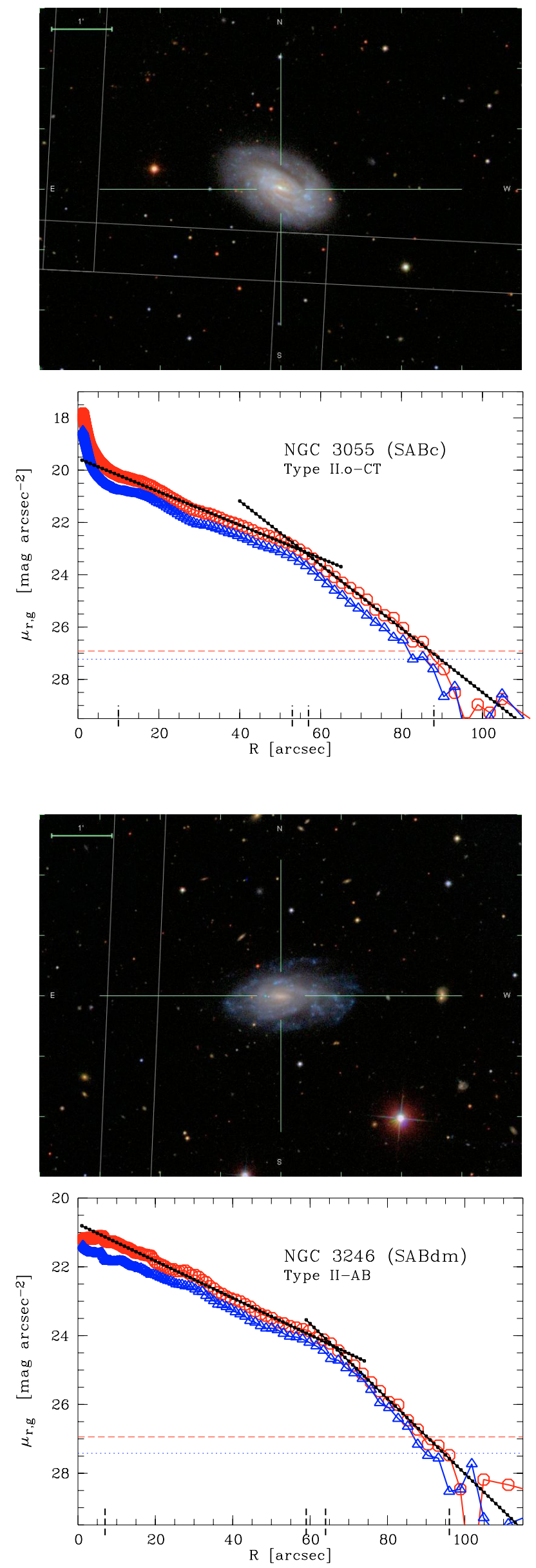
NGC 3259: Type III-d

J103234.8+650228.SXT4* $4.0-19.842 .11929$

The background is rather disturbed by a bright star, but the galaxy is small enough not to be significantly influenced. Galaxy is isolated according to Prada et al. (2003), but has one SDSS-detected dwarf companion $\left(\sim 18.0 \mathrm{~g}^{\prime}\right.$-mag) about $10^{\prime}$ away with similar velocity $\left(v=1744 \mathrm{~km} \mathrm{~s}^{-1}\right)$ and another possible, dwarf companion (or large $\mathrm{H}$ II region) projected on the south part of the disk (no counterpart in NED), which is partly covered by a foreground star, but also clearly present on the DSS image. The break region of the upbending profile at $\sim 55^{\prime \prime}$ is fairly extended and curved. The inner profile shows wiggles at $\sim 15^{\prime \prime}$ and $\sim 30^{\prime \prime}$, but the resolution is too low to identify a bar or ring. The spiral structure apparently extends into the outer disk well beyond the break, with two, thin symmetric spiral arms (plus more fluffy ones) starting at the defined break radius. However, it is not clear if they are really the continuation of the inner arms. The upbending characteristic is confirmed by a profile shown in Courteau (1996) (see UGC 05717).

NGC 3310: Type III

J103845.8+533012 .SXR4P $4.0-20.252 .81208$

The galaxy is clearly disturbed with a shell-like structure in the outer disk, which is probably the result of a recent merger with a smaller galaxy (cf. Conselice et al. 2000). The inner region is also slightly asymmetric, but still used for centering. The inner region, corresponding to a high surface brightness, tightly wound spiral, inside the break at $\sim 50^{\prime \prime}$, is curved starting at $\sim 20^{\prime \prime}$. The wiggles in the outer disk are due to the shells. An additional upbending beyond $\sim 150^{\prime \prime}$ is due to a prominent outer shell. The very outer parts are in addition influenced by an inhomogeneous sky and a large mask.
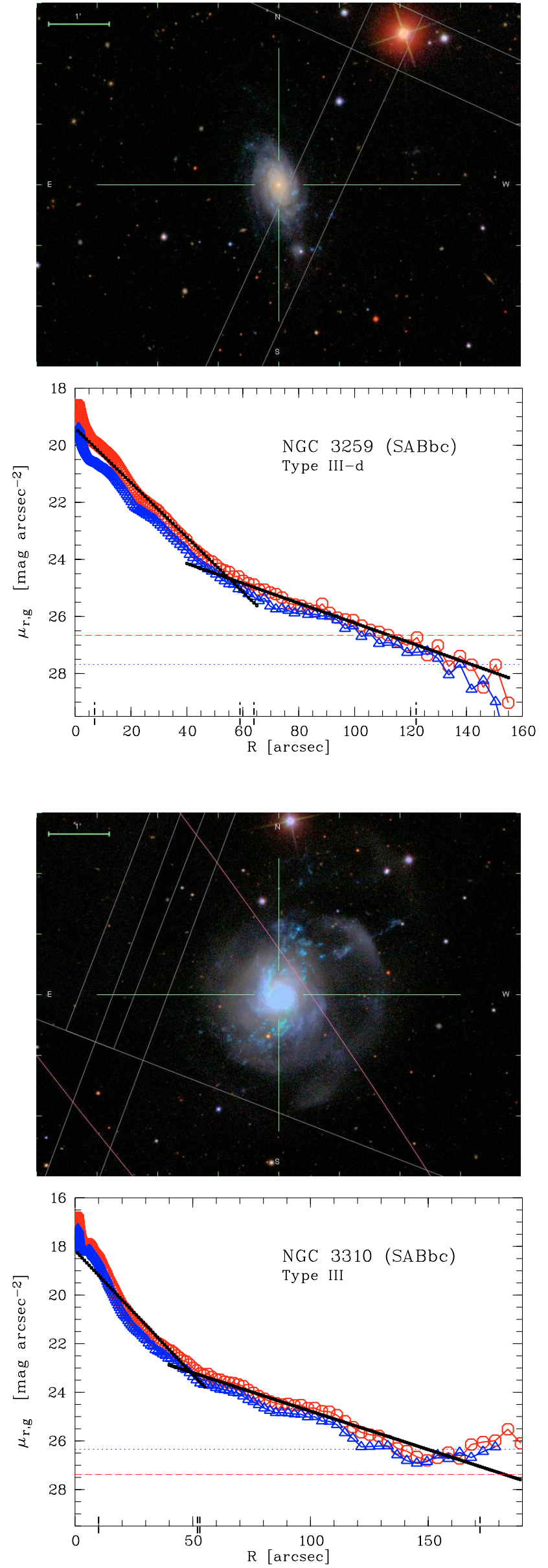
NGC 3359: Type II-AB

J104636.3+631328.SBT5. $5.0-20.427 .21262$

The inner disk shows an extended bar (roughly $R \sim 45^{\prime \prime}$ ), which is not visible in the final profile, and two bright spiral arms. The broad extension of this arms build the outer disk, so there is no clear elliptical outer structure visible which makes the photometric inclination (ellipticity) and PA measurements highly uncertain. The outer slope is influenced by a rather uncertain sky estimate in both bands, but the apparent downbending break at $\sim 200$ "is not due to a sky error, but coincides with the region where the two outer arms dominate. Thus the galaxy is classified as Type II-AB.

NGC 3423: Type II-CT

J105114.3+055024 .SAS6. $6.0-19.54 \quad 3.91032$

Bulge-like inner region $\left(\$ 30^{\prime \prime}\right)$ coincides with inner high surface brightness disk with spiral structure. Final profile is clearly downbending but the exact break radius is difficult to place due to an extended feature around $\sim 70^{\prime \prime}$ corresponding to an aligned spiral arm (with a possible straight structural element towards the north-east, called (Chernin 1999) spiral-arm row).
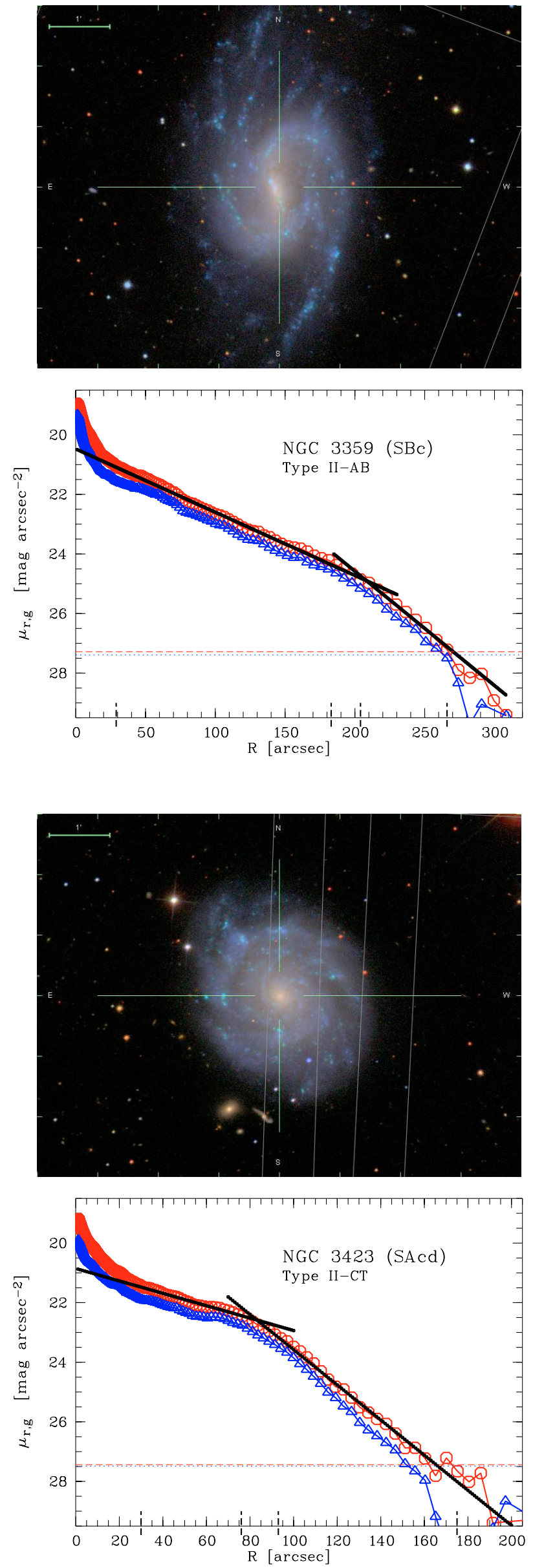
NGC 3488: Type II.o-CT

J110123.6+574039.SBS5* $5.1-18.801 .63226$

Galaxy is isolated according to Prada et al. (2003). A bright star covering part of the outer disk needs an extended mask. A small bar of roughly $R \sim 6^{\prime \prime}$ size is visible as an elongated isophote on the image. The final profile is clearly downbending, but the exact break radius is difficult to place. The extended break region resembles in this case again a straight line, thus one could also define two break radii at $\sim 32 "$ and $\sim 58^{\prime \prime}$. This could be caused by the extended mask, whereas the spiral structure extending to $\sim 40^{\prime \prime}$ seems not to be responsible for this behaviour. The bar is to small for its OLR to be associated with the first break, thus the galaxy is classified Type II.o-CT. The light beyond $\sim 58^{\prime \prime}$ is still symmetric and not affected by sky errors. The peak at $\sim 20^{\prime \prime}$ corresponds to the inner two arms.

NGC 3583: Type III-d

J111411.0+481906.SBS3. $3.2 \quad-19.572 .32347$

The apparent companion, a spiral galaxy (NGC 3577) towards the south-west is non-physical with $v=5336 \mathrm{~km} \mathrm{~s}^{-1}$. The galaxy shows a stream-like arm extending towards the northwest outside of the galaxy connected to a small superimposed E0 satellite at $0.9^{\prime}$ north (not in NED). Due to this structure the outer disk seems to have a slightly different ellipticity and PA. The bump at $\sim 30^{\prime \prime}$ in the final profile is due to the end of the bar and beginning of spiral arms. The inner profile with an extended wiggle at $\sim 55^{\prime \prime}$ (corresponding to the spiral arms) makes it difficult to characterise the break region (starting at $\sim 85^{\prime \prime}$ with an upbending profile) as being curved or sharp.
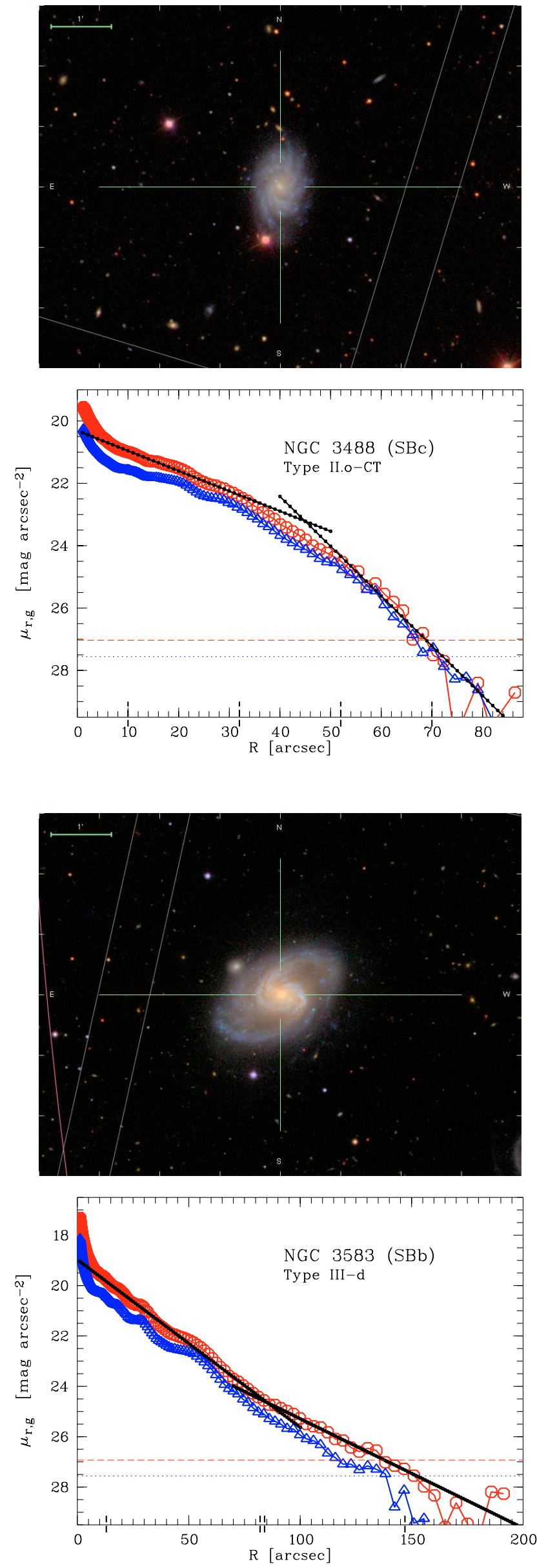
NGC 3589: Type II-CT

J111513.2+604201 .S. .7* 7.0 -18.491 .52217$

Due to the higher inclination and extended elongated structure the size of the bar is not well defined. The center is also not well defined, but centering on the outer isophote coincides roughly with the brightest pixel. The sky estimate is rather uncertain due to the bright star in the FOV. The final profile shows a downbending break at $\sim 40^{\prime \prime}$, classified as Type IICT but also consistent with being Type II.o-OLR allowing the bar to be of size $\sim 20^{\prime \prime}$ which is consistent with the image. The small bump $\sim 22^{\prime \prime}$ in the profile just corresponds to some aligned $\mathrm{H}$ II regions.

NGC 3631: Type I

J112102.4+531008 .SAS5. $5.1 \quad-20.62 \quad 4.91388$

The galaxy forms a nearby group together with NGC 3178, NGC 3898, NGC 3953 and NGC 3992 (also part of our sample) in the Ursa Major cluster and shows plenty of substructure. It has two prominent spiral arms with different pitch angles, one of them turns nearly straight, classified by Arp (1966) as a spiral galaxy with one heavy arm. In addition, it has a faint extended spiral arm (or maybe stream-like structure) in the far outer disk towards North, therefore the outer disk is slightly asymmetric and the mean ellipticity and PA are difficult to fix. There is an extended light patch on the image visible towards south-west. Although there is no bright star nearby which could create such an artefact it is unlikely a dwarf companion, since it is not visible on the DSS. The final radial light profile is quite unusual and cumbersome to classify, since it exhibits an extended bump between 50-140", which corresponds to the begin of the two spirals to the end of the inner (undisturbed) disk. Beyond $\sim 140 "$ the extended, slightly offcenterd outer disk with the spiral arm structure (or stream) starts. If we do not exclude the bump from the fit, one can argue for a downbending break at $\sim 75^{\prime \prime}$, or a Type I profile with an extreme wiggle (this would be a Type II.i if the galaxy would be barred). We decided to exclude the bump and the inner region from the fit $\left(R \gtrsim 140^{\prime \prime}\right)$ which gives also a Type I profile where the region around $\sim 35^{\prime \prime}$ is also roughly fitted. Due to this problems the classification should be taken with caution.
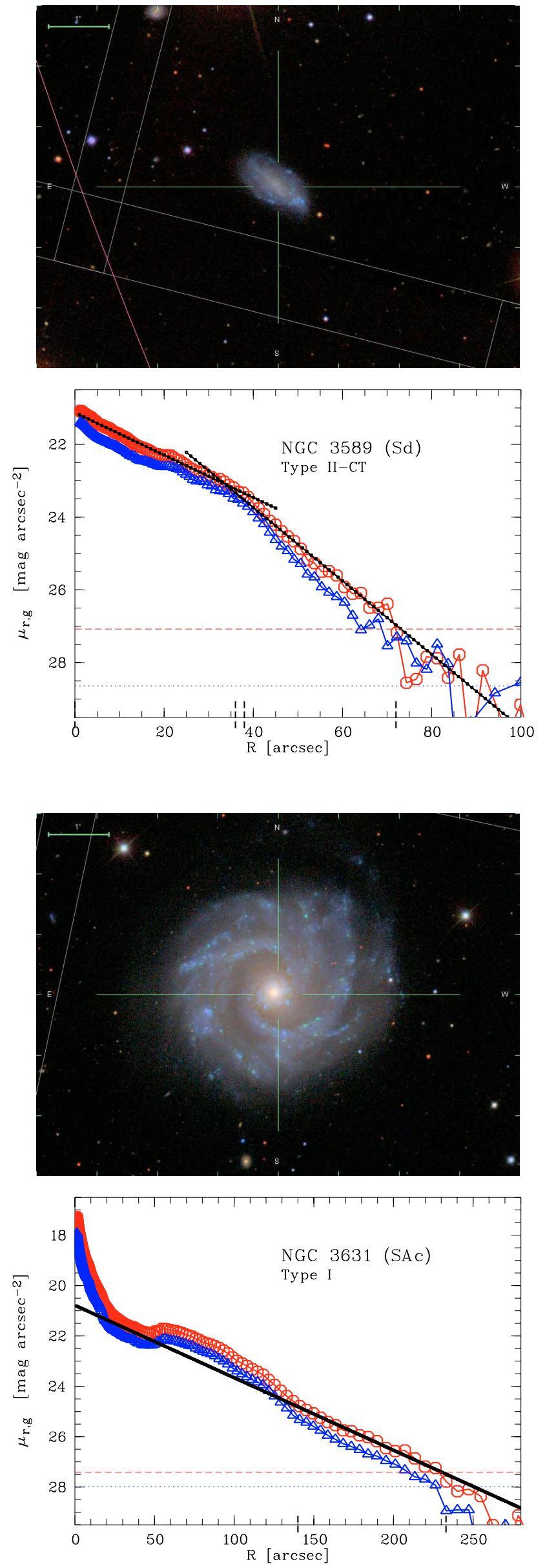
NGC 3642: Type III-d

J112218.0+590427 .SAR4* $4.0 \quad-20.605 .51831$

The background is slightly inhomogeneous and the galaxy is rather extended. From the nearly round inner disk a single, star forming, spiral arm starts towards the outer disk with slightly more than one revolution, forming the strongly asymmetric, lopsided outer disk. Therefore the ellipticity and PA is determined further inside. The final profile looks fairly curved and with extended wiggles in the outer disk at $\sim 100^{\prime \prime}$ and $\sim 155^{\prime \prime}$ due to the outer spiral arm passing the ellipse. There is a steeper inner exponential part visible between $\sim 25-75^{\prime \prime}$ followed outwards by an upbending profile, which is roughly at the position where the extended spiral arm starts to unwrap, which leads to the Type III classification. Inwards of $\sim 25^{\prime \prime}$ there is again another steeper exponential part visible together with a possible bulge component.

NGC 3756: Type II.o-CT

J113648.3+541737.SXT4. $4.3 \quad-19.893 .81525$

Only partly fitted since $\lesssim 1 / 4$ of galaxy is beyond field. Known three armed spiral galaxy which shows one wide spiral arm starting at $\sim 60^{\prime \prime}$ extending roughly towards the downbending break at $\sim 100^{\prime \prime}$. The extended bump $\sim 45^{\prime \prime}$ is due to the three inner tightly wound spiral arms and makes it, similar to NGC 3631, difficult to fit the inner region. However, since the bump is less strong we decided to include it. The galaxy is classified as SAB (RC3) but the bar is not obvious on the image and its maximum size would be $R \lesssim 25^{\prime \prime}$ thus the break is well outside the range for typical Type II.o-OLR breaks and is classified as Type II.o-CT.
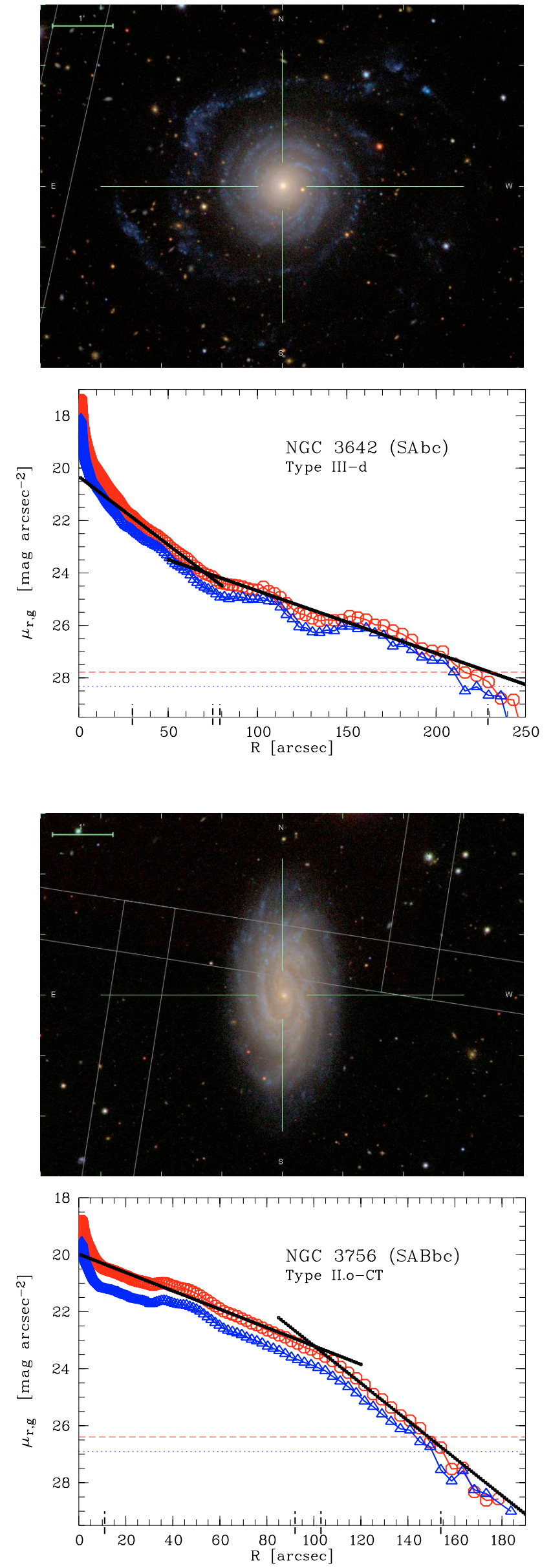
NGC 3888: Type I

J114734.7+555800.SXT5. $5.3 \quad-20.43 \quad 1.82648$

The background on the image shows a large gradient due to a bright star off the field, but the galaxy is small enough to be not affected. The galaxy has one "malformed" (kinked) spiral arm across the disk. There is a possible dwarf, irregular companion visible towards the south-west, but without any distance information. The final profile exhibits an extended wiggle (with an unusual integral sign shape) at $\sim 33^{\prime \prime}$, looking like two shifted exponential regions, but there is no point in placing a break there. The peak at $\sim 22^{\prime \prime}$ is related to an aligned spiral arm, whereas the peak at $\sim 12^{\prime \prime}$ could be due to the end of a possible bar or inner ring (no obvious on the image). Therefore the galaxy is classified as Type I and not as Type II.i, although the dip in the profile is clearly visible. It could be explained by dust lanes close to the center, which also explains the dip being more prominent in the $g^{\prime}$ band.

NGC 3893: Type III-d

J114838.4+484234.SXT5* $4.8-20.334 .11193$

The background around the galaxy is affected by a nearby, bright star and a close (3.7' away, with similar velocity $v=905 \mathrm{~km} \mathrm{~s}^{-1}$, SB0/a:pec), smaller, companion galaxy (NGC 3896), both members of the Ursa Major Cluster. Galaxy exhibits a wide, extended spiral arm like structure towards the east, which could also be a stream or tidal feature. Due to this structure it is not possible to derive the ellipticity and PA from the outer disk, so their values are more uncertain. The final profile clearly shows an Type III upbending profile starting at $\sim 150^{\prime \prime}$ corresponding to the beginning of the extended structure to one side and some more symmetric faint light on the other side. The inner disk shows a prominent wiggle $\sim 90^{\prime \prime}$ which is associated to a pseudoring of wrapped spiral arms.
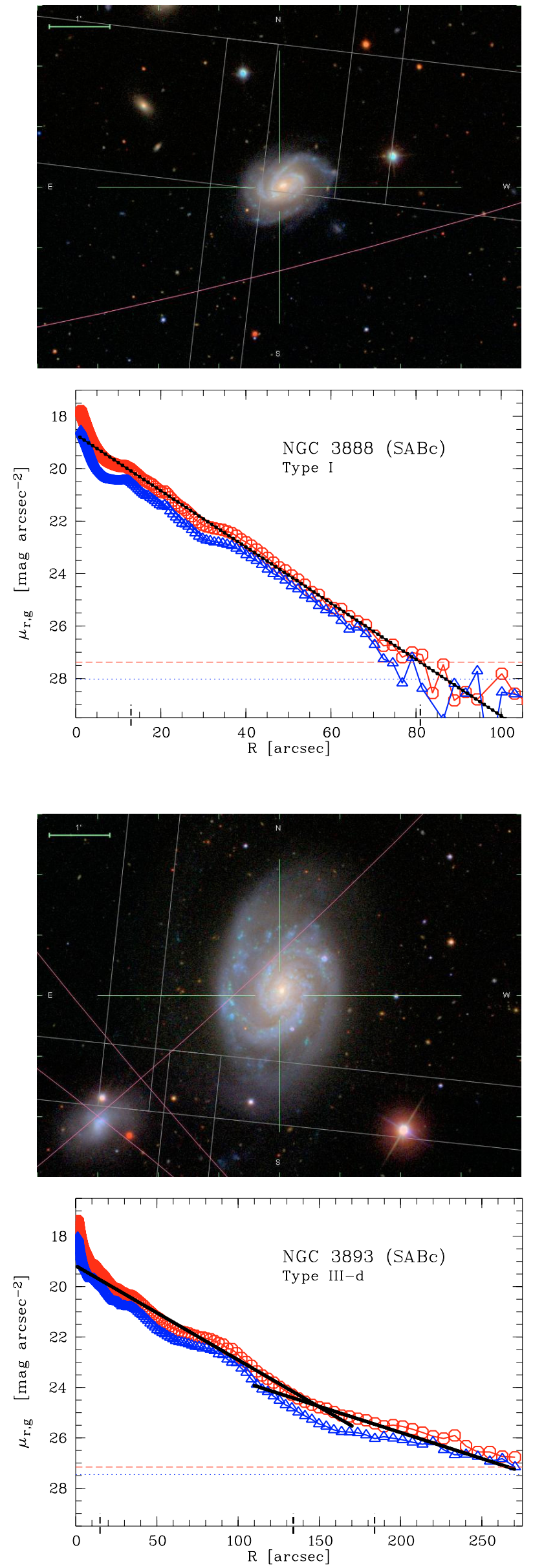
NGC 3982: Type III

J115628.3+550729.SXR3*3.2 -19.54 2.31351

Close to Ursa Major Cluster but membership questionable (Tully et al. 1996). Galaxy exhibits a detached, narrow, starforming spiral arm embedded into the very outer, symmetric disk towards south-west. The final profile beyond the central part is smooth and straight down to $55^{\prime \prime}$ where it clearly starts upbending, classified as Type III, which is consistent with the profile by (Tully et al. 1996). The break corresponds to the end of the inner disk, excluding the faint outer arm, which produces the bump at $\sim 75^{\prime \prime}$ (more pronounced in the $g^{\prime}$ band). The transition zone is rather sharp. Dip-peak structure between $\sim 5-12^{\prime \prime}$ corresponds to the inner bar/ring region. The exact size of the bar is not well determined on the SDSS image. Erwin (2005), using HST images, gives 5" and classifies the galaxy therefore as Type II.o-OLR + III (with an "extreme" OLR break). We exclude the inner $R \lesssim 13$ " from our fit and leave it with a Type III classification.

NGC $3992 \equiv$ M 109: Type II.o-OLR

J115735.9+532235.SBT4. $3.8-20.986 .91286$

Large galaxy with a small part off the SDSS field. Member of Ursa Major Cluster (Tully et al. 1996). Embedded in the large bar of size roughly $R \sim 70^{\prime \prime}$ (as measured on the image) is possibly a secondary bar half the size. Unusual very faint extension visible in both bands (more pronounced in $g^{\prime}$ band) towards north-east, which is possibly but unlikely straylight from a nearby bright, red star. The one-sigma ellipse is therefore not representative so the mean ellipticity and PA are determined further inside. Final profile shows a clear downbending break at $\sim 140^{\prime \prime}$ at about twice the bar radius corresponding roughly to a pseudoring of the spiral arms, therefore classified as Type II.o-OLR. Bump at $\sim 200^{\prime \prime}$ does not coincide with a spiral arm.
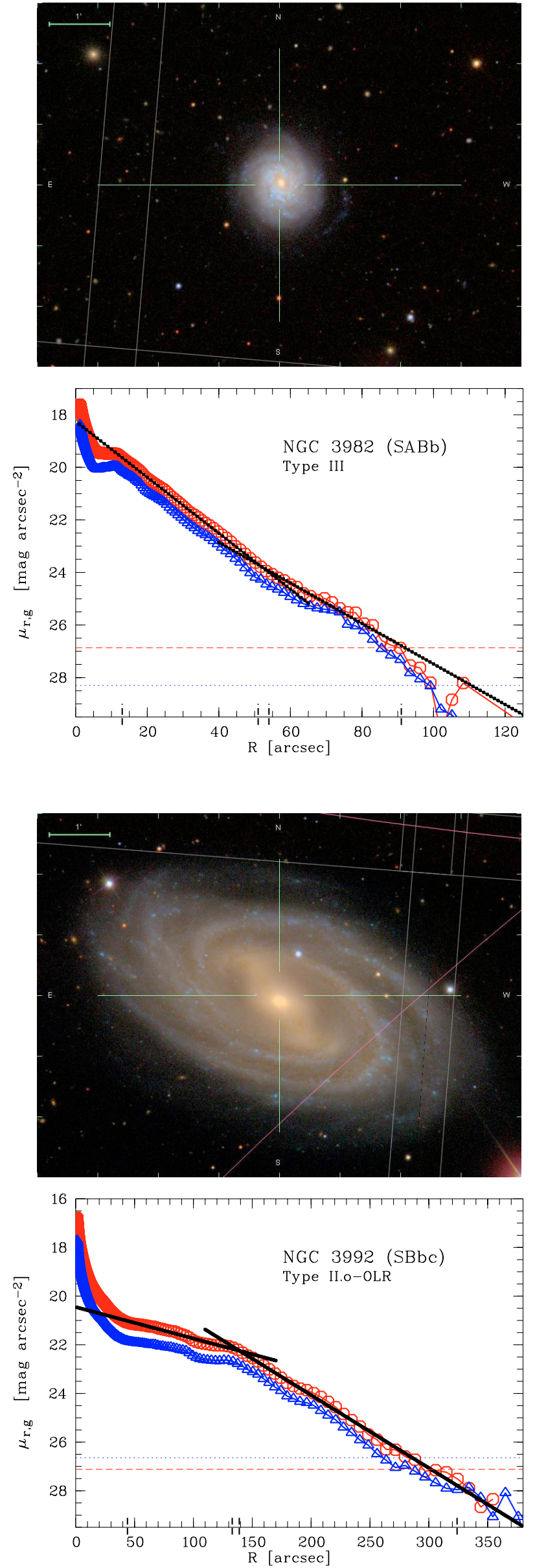
NGC 4030: Type III

J120023.4-010603.SAS4. $4.1-20.27 \quad 3.91476$

Two superimposed bright stars are masked. According to Zaritsky et al. (1993) it is an isolated galaxy with a satellite system (largest UGC 06970, SB(s)m, with $M_{\text {abs }}=-17.7$ ). The galaxy shows two prominent spiral arms beyond a more flocculent center starting at $\sim 30^{\prime \prime}$, followed by a single arm extending slightly further out towards south-west. Final profile shows a Type III break at $\sim 150^{\prime \prime}$ where the transition zone is rather sharp, followed by a fairly symmetric outer region without spiral structure. The bump at $\sim 80^{\prime \prime}$ corresponds to the end of inner spiral arms.

NGC 4041: Type III-d

J120212.2+620814 .SAT4* $4.3-19.92 \quad 2.71486$

According to Sandage \& Bedke (1994) this galaxy forms a kinematic triplet with NGC 4036 and UGC 7009 and is probably member of the Ursa Major Cluster. However, Tully et al. (1996) does not list any of these as members. Galaxy shows a detached, extended, star-forming spiral arm structure in the outer, asymmetric disk, similar to NGC 2967, but smaller and only one-sided, which makes the photometric inclination (ellipticity) and PA uncertain. In the final profile the disk inside the Type III break at $\sim 75^{\prime \prime}$ corresponds roughly to the region with the spiral arms, which are also responsible for the extended bump between $\sim 30-75^{\prime \prime}$ (more pronounced in $g^{\prime}$ band). The very inner region looks bar-like, but is probably only the spiral structure continuing into the center.
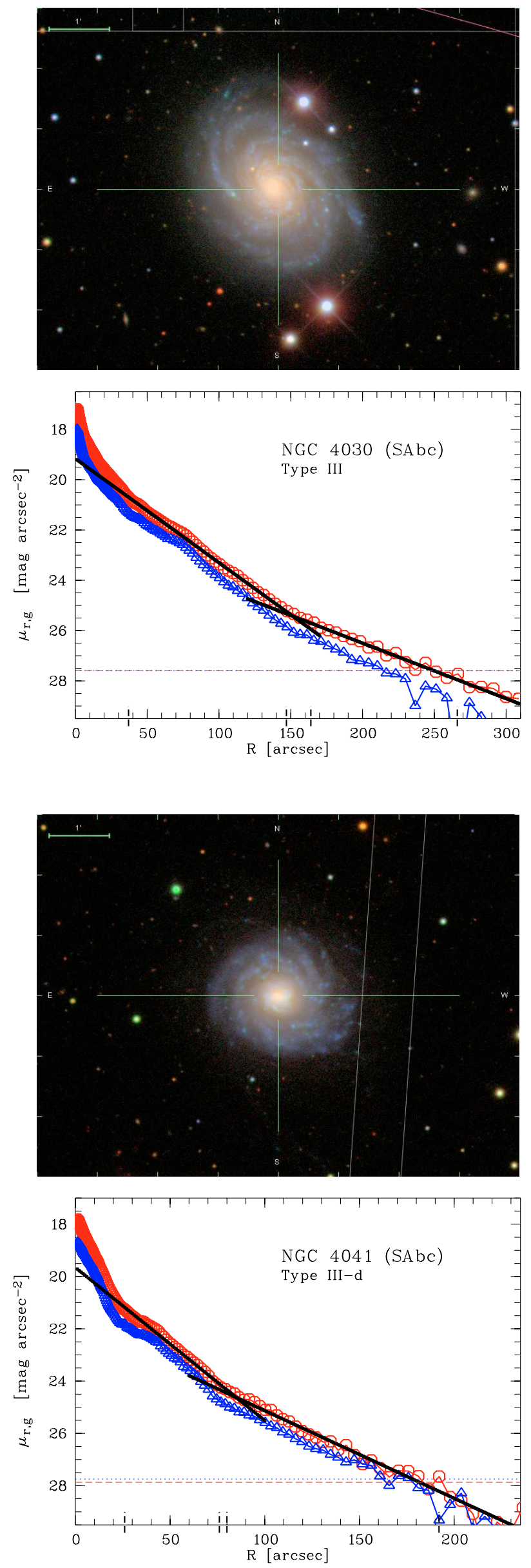
NGC 4102: Type II.o-OLR + III

J120623.1+524239.SXS3\$ $3.0-19.383 .11082$

Galaxy belongs to the Ursa Major Cluster Tully et al. (1996). On the image an elongated inner bar region inside a starforming ring is visible which is responsible for the dip and bump structure between $\sim 25-35^{\prime \prime}$ in the final profile. Erwin (2005) measures the size of the bar to $\sim 15^{\prime \prime}$ and the outer ring to $\sim 35^{\prime \prime}$, so the apparent break at $\gtrsim 40^{\prime \prime}$ (although measured from outside, it starts only at $\left.\sim 58^{\prime \prime}\right)$ is classified as a Type II.oOLR. The apparent upbending at $\sim 120^{\prime \prime}$ is unlikely a sky error, visible around the whole galaxy and confirmed by the profile in Courteau (1996) (see UGC 07096) and also visible in Erwin et al. (2006). This adds the Type III classification.
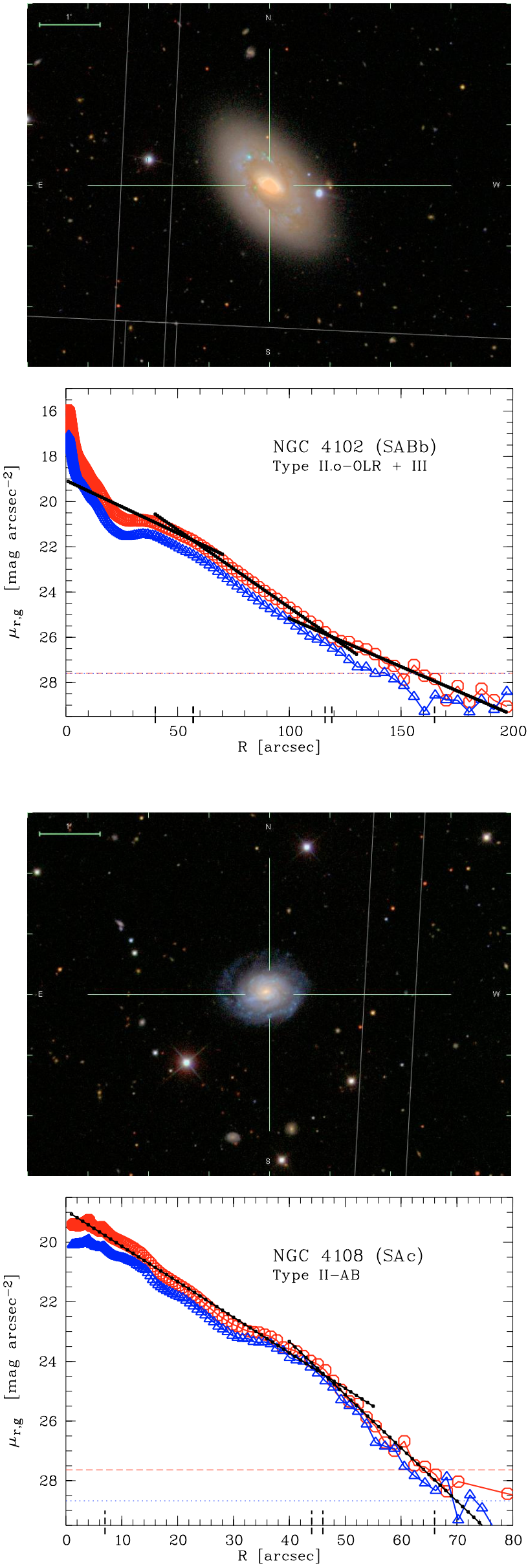

NGC 4108: Type II-AB

J120644.0+670944 PSA. $5 * 5.0-20.131 .72828$

Galaxy in a triple system with NGC 4108A $\left(v=2256 \mathrm{~km} \mathrm{~s}^{-1}\right)$ and NGC 4108B $\left(v=2673 \mathrm{~km} \mathrm{~s}^{-1}\right)$, the latter is also part of the present sample. According to Nordgren et al. (1997) NGC 4108 and NGC 4108B appear to be possibly joined by an H I bridge. Very inner center $\lesssim 5^{\prime \prime}$ looks like small bar, or the spiral structure starts from the very center. The outer disk is asymmetric with one side rather sharp and the other more shallow with indication for a single spiral arm. The disk is lopsided, so the outer contour is used for centering (center $\sim 4$ " off the brightest pixel, causing the central dip in the profile), which implies that the photometric inclination (ellipticity) and PA are not well defined. The apparent break at $\sim 44^{\prime \prime}$ is related to the transition between the symmetric outer disk going inwards to the offcenterd inner disk and therefore most probably not intrinsic but a Type II-AB break. 
NGC 4108B: Type I

J120711.3+671410.SXS7P $7.0-18.46 \quad 1.32840$

Companion to NGC 4108 (see above). Galaxy exhibits an offcentered bar $\left(\sim 5^{\prime \prime}\right.$ off compared to the center derived from the outer isophote). Very outer disk slightly asymmetric towards north-east (outer spiral arm?). In the final profile the central dip and inner wiggle are due to the the offcentered bar, the peak at $\sim 22^{\prime \prime}$ corresponds to an aligned spiral arm-like region. Although a downbending break at $~ 32$ " with low scalelength contrast is possible, Type II-AB, the galaxy is consistent and classified as Type I due to the wiggles in the center.

\section{NGC 4123: Type I}

J120811.1+025242 .SBR5. 4.8 -19.55 4.11364

Galaxy with extended bar $\left(R \sim 55^{\prime \prime}\right)$ studied in detail by Weiner et al. (2001) using a surface brightness profile which is consistent with the SDSS one. The outer disk is slightly asymmetric, more rectangular than elliptical, with an extended starforming spiral arm structure towards south-west, which makes the photometric inclination (ellipticity) and PA uncertain. The final profile exhibits extended wiggles. The bar and associated spiral arms are visible between $\sim 30-110^{\prime \prime}$ and the bump at $\sim 170^{\prime \prime}$ corresponds to a starforming outer spiral arm. Although being far from a prototypical Type I we used this classification, since the profile shows too many changes to reasonably argue for any other type.
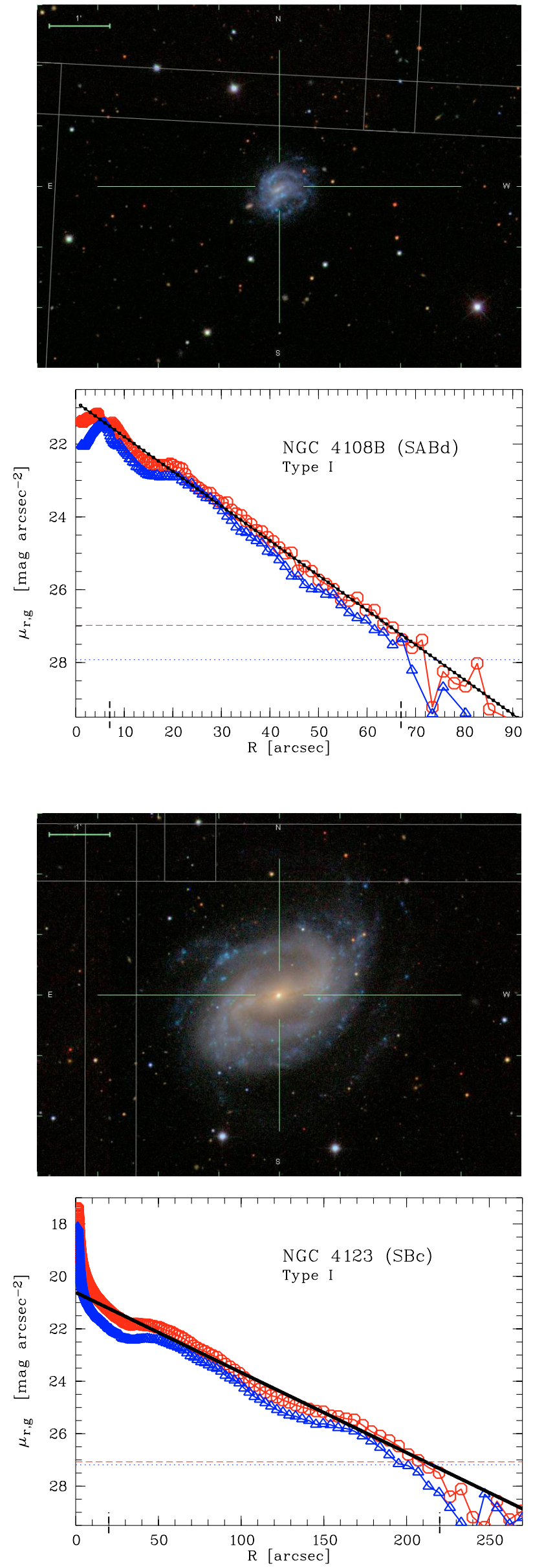
NGC 4210: Type II.o-OLR + II.o-CT

J121515.9+655908.SBR3. $3.0-19.892 .03000$

Companion galaxy (CGCG 315-030, $m=15.5) 13^{\prime}$ away with similar velocity $\left(v=2679 \mathrm{~km} \mathrm{~s}^{-1}\right)$. Gradient in the background due to a very bright star (off field), but galaxy small enough to be not significantly influenced. The final profile shows an inner bump at $\sim 15^{\prime \prime}$ corresponding to a pseudoring of wrapped spiral arms (slightly further out than the bar). The apparent dip $\sim 8^{\prime \prime}$ is due to the bar orientation being perpendicular to the major axis of the galaxy. Similar to NGC 3488 the profile shows a clear downbending. However, the break radius is uncertain due to the extended break region, which resembles in this case again a straight line. Thus one could also define two break radii at $\sim 33^{\prime \prime}$, corresponding roughly to end of inner spiral arm structure, and $\sim 55^{\prime \prime}$, not related to sky errors and symmetric around the disk. In contrast to NGC 3488 the bar is here large enough to argue for a combination of a Type II.o-OLR and a Type II.o-CT break, this should be confirmed with a more detailed study on the bar size of NGC 4210.

NGC 4273: Type II-AB + III

J121956.2+052036 .SBS5. $5.1-20.412 .12435$

Galaxy in south-western corner of the Virgo Cluster with three similar sized neighbours, NGC 4268, NGC 4281, and closest and slightly overlapping at the very outer disk NGC 4277. The disk is lopsided with one side sharp edged and the other side with a wide spiral arm feature towards south-east (embedded in the outer disk), similar to NGC 4108. Centering on the outer disk causes the dip at $\sim 10^{\prime \prime}$ and hides the very bright nucleus sitting in a bar-like structure which is offcentered by $\sim 16^{\prime \prime}$. The break at $\sim 45^{\prime \prime}$ in the final profile is clearly related to the lopsided disk and therefore classified as Type II-AB. In addition, there is an Type III break with an upbending profile visible beyond $\sim 80^{\prime \prime}$ due to the influence of the companion (possibly intrinsic and not just projected).
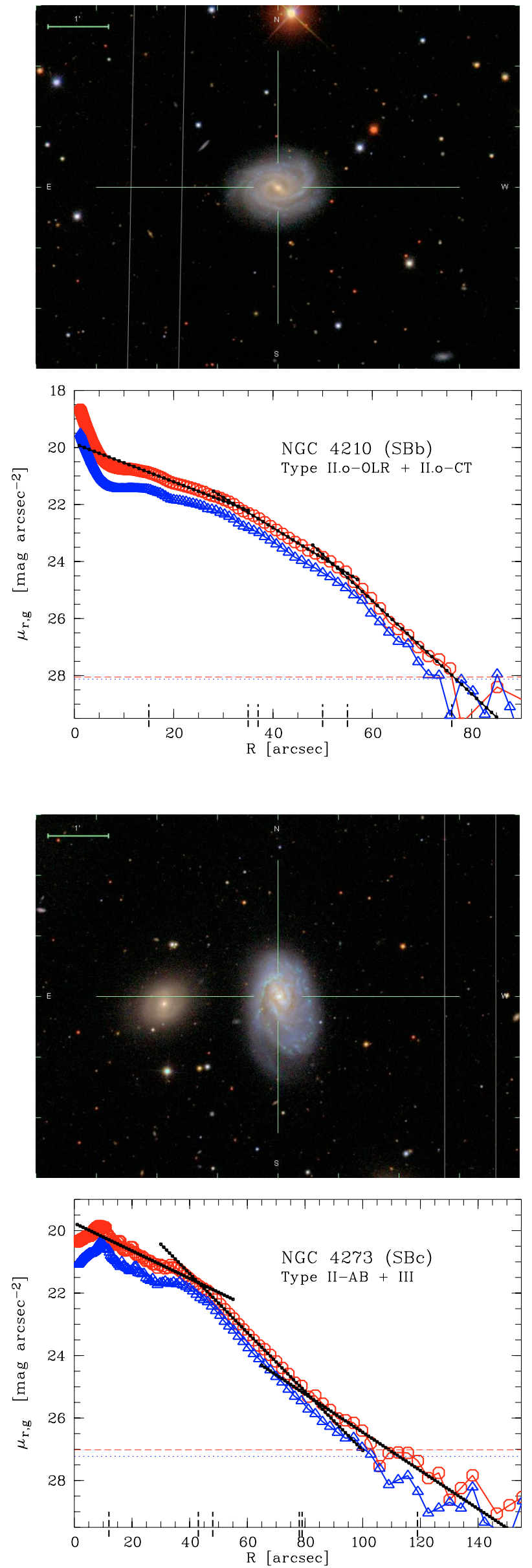
NGC 4480: Type II.o-CT

J123026.8+041447.SXS5. $5.1-20.142 .12494$

The galaxy is small, rather inclined, and classified as SAB with bar size of roughly $R \sim 10^{\prime \prime}$ (measured from the image). The final profile shows a clear downbending break at $\sim 55^{\prime \prime}$, significantly further out compared to a typical Type II.o-OLR break thus classified as Type II.o-CT, corresponding roughly to the end of the spiral arms. The inner profile exhibits an extended bump at $\sim 35^{\prime \prime}$ (more pronounced in the $g^{\prime}$ and less in the $i^{\prime}$ band) most probably related to the star formation in the spiral arms and not to a possible Type II.o-OLR + II.oCT as for NGC 4210, which shows the same structure in both bands.

NGC 4517A: Type II.o-CT + II.o-AB

J123228.1+002325.SBT8* $7.9-19.183 .81562$

This low surface brightness galaxy has an elongated, barred center, of size $R \sim 30^{\prime \prime}$, with a foreground star very close and a slightly asymmetric, lopsided outer disk. The final profile exhibits a sharp break at $\sim 150^{\prime \prime}$ due to the asymmetric outer disk, which is not well described with the mean ellipticity and PA used, thus classified as Type II-AB. There is an additional feature (bump or break) visible around $\sim 80^{\prime \prime}$, which is not obviously related to an aligned spiral arm and could be explained by a weak downbending break. Since the bar appears to be only weak and the break at more than twice the bar radius it is classified as Type II.o-CT, which should be taken with caution.
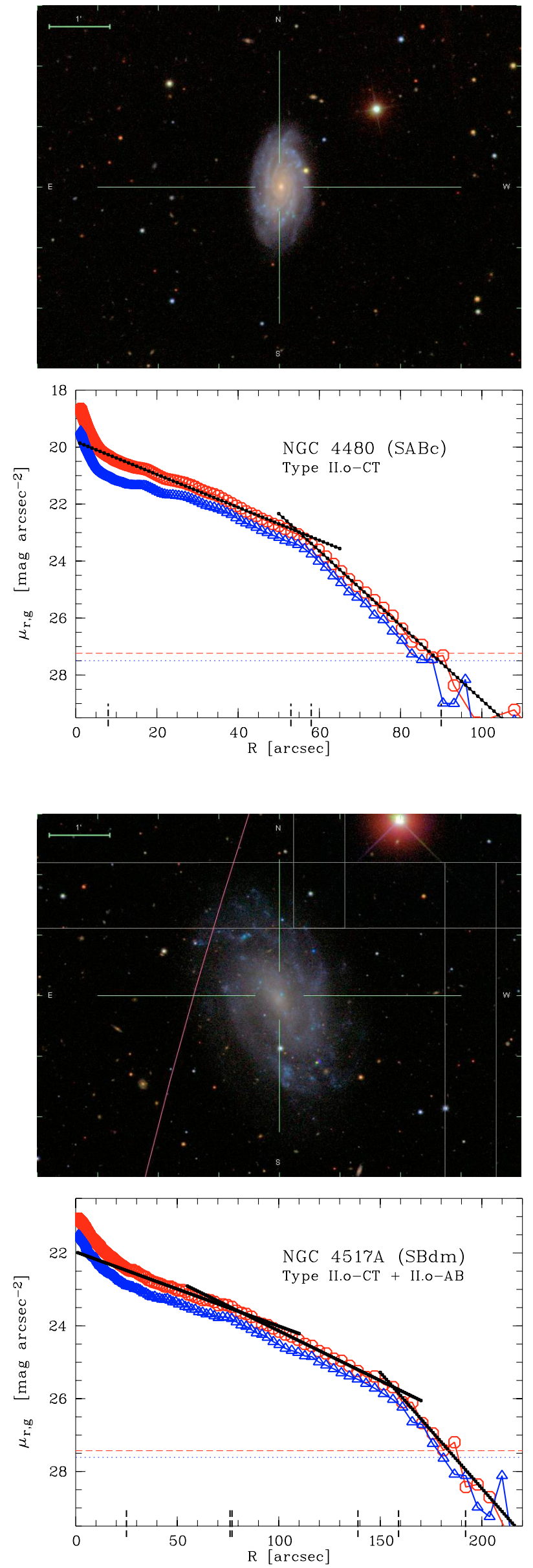
NGC 4545: Type II.o-CT

J123434.2+633130.SBS6*5.9 -20.492 .53000$

Small galaxy with an s-shaped inner region of size $\sim 12^{\prime \prime}$ (associated to be the bar) leading to two (or three) tightly wrapped, spiral arms. Final profile exhibits a break at $58^{\prime \prime}$, being too far out for a typical Type II.o-OLR thus classified as Type II.o-CT. The dip at $\sim 8^{\prime \prime}$ corresponds to the region where the spiral arms start from the bar-structure which is nearly perpendicular to the ellipse major axis.

NGC 4653: Type II-AB

J124350.9-003341.SXT6. $6.1-20.373 .02658$

Paired galaxy with NGC $4642\left(v=2471 \mathrm{~km} \mathrm{~s}^{-1}, \sim 10^{\prime}\right.$ away $)$ while apparent dwarf companion on the image towards southeast is a confirmed background galaxy $\left(v=7843 \mathrm{~km} \mathrm{~s}^{-1}\right)$. Extended mask used to avoid straylight by bright stars. Mean ellipticity and PA difficult to determine, since it is different for the inner disk (used for centering) with two, very regular spiral arms inside $\$ 30^{\prime \prime}$, compared to the outer disk with more frayed spiral fragments, starting to be asymmetric on larger scales at $\lessgtr 80^{\prime \prime}$ (mainly towards south-west). This asymmetry causes also the apparent break at $\sim 105^{\prime \prime}$ thus the galaxy is classified as Type II-AB.
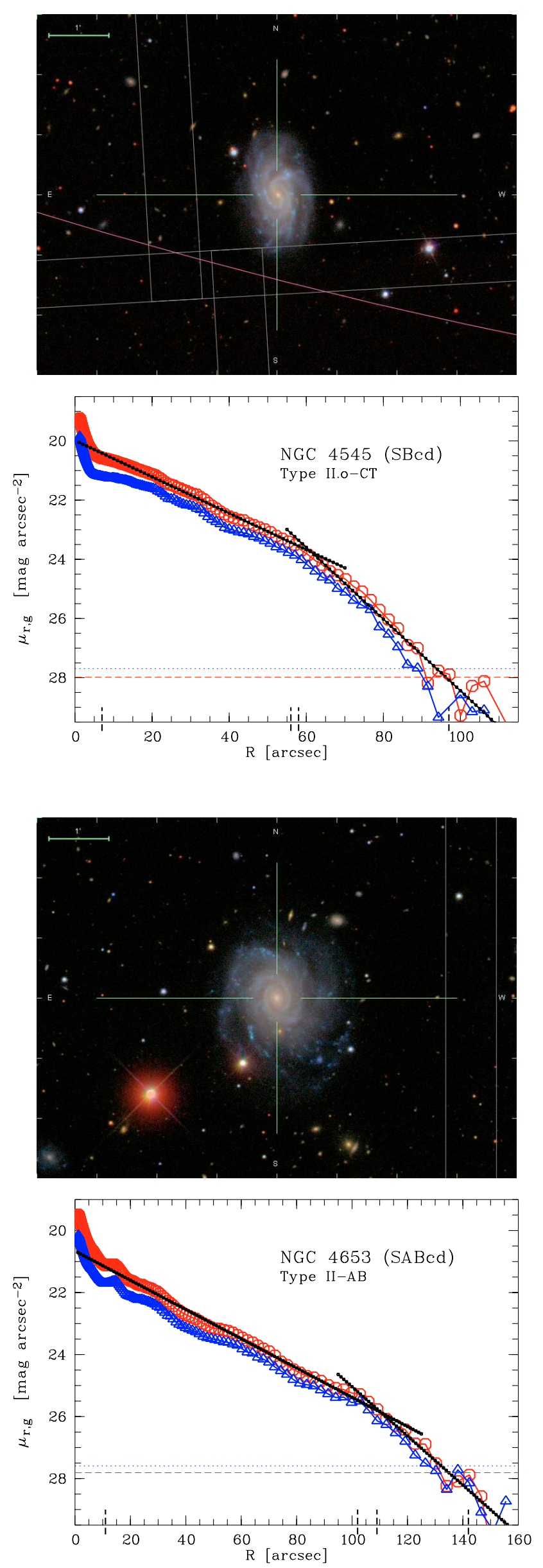
NGC 4668: Type III

J124532.0-003209.SBS7*7.1 -18.88 1.51654

Small galaxy close to our high axis ratio limit, which forms a close pair with NGC $4666\left(v=1474 \mathrm{~km} \mathrm{~s}^{-1}\right)$ about $7^{\prime}$ away and also has a wider association with NGC $4632(v=$ $1557 \mathrm{~km} \mathrm{~s}^{-1}$ ) according to Sandage \& Bedke (1994). The final profile exhibits a break at $\sim 70^{\prime \prime}$ followed by an upbending profile corresponding to a slightly asymmetric, faint outer disk and although close to, most probably not related to a sky error, thus classified as Type III. The full extend of the bar is uncertain, although the bump around $\sim 20^{\prime \prime}$ is certainly related to the bar and a possible spiral arm. The whole inner region is included in the inner disk fit.
NGC 4904: Type II.o-OLR + III

J130058.6-000138 .SBS6. $5.7-18.692 .11213$

Only partly fitted since $\lesssim 1 / 5$ of galaxy is beyond field. The galaxy has a dominating, thick bar of size $R \gtrsim 20^{\prime \prime}$ whereas the center along the bar is not well defined (centering on brightest pixel). It has an unusual spiral arm structure with one extending nearly straight towards south-west forming more droplet shaped isophotes. The image shows indication for an additional, extremely faint, extended patch of light outside the disk towards south-west. Final profile shows a clear downbending break at $\sim 40^{\prime \prime}$, associated on one side with a ring-like structure which is about twice the bar radius, thus classified as Type II.o-OLR. The apparent upbending at $\sim 90^{\prime \prime}$ is almost certainly produced by the additional patch of light unrelated to any artificial source, thus adding the Type III classification.
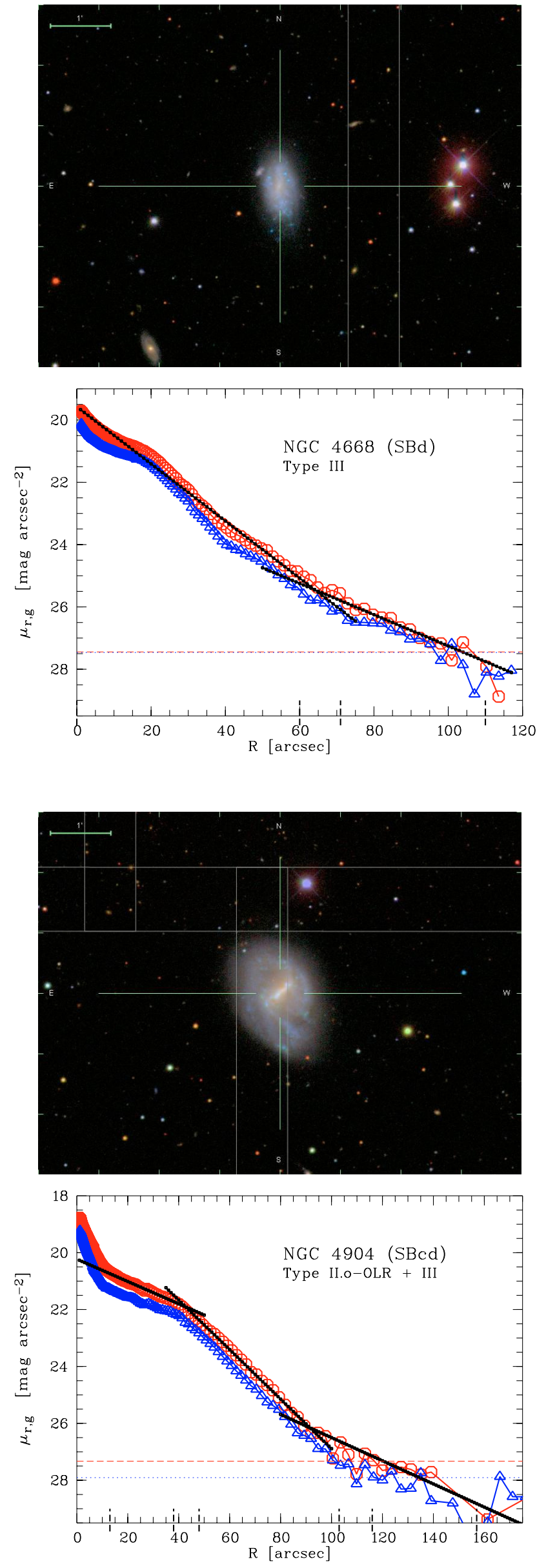
NGC 5147: Type II.o-OLR + III

J132619.2+Q20604 .SBS8. $7.9-18.77 \quad 1.81154$

The medium-sized star superimposed on the galaxy close to its center is masked. The bar size is uncertain but could be associated to an elliptical region of size $R \lesssim 15^{\prime \prime}$ on the image not reflected in the final profile. The extended bump between $\sim 25-$ $45^{\prime \prime}$ is associated to the inner disk containing the spiral arms with some ring-like, distributed SF regions. Excluding the full region allows for a crude Type I classification. However, using a break at $\sim 30^{\prime \prime}$ (about twice the bar radius thus classified as Type II.o-OLR) followed by a downbending profile with another break at $\sim 75^{\prime \prime}$ leading to an upbending profile (therefore classified as Type III) seems more reasonable, even so the upbending is very close to our detection limit. The disk beyond the ring-like structure is slightly asymmetric with a single, wide, and faint spiral arm-like structure towards north-east just inside the outer break. The outermost isophote is again nearly round and symmetric.

\section{NGC 5300: Type II.o-CT}

J134816.1+035703 .SXR5. $5.0-18.93 \quad 3.51246$

Galaxy close to border of SDSS field but almost complete. The size of the weak bar is about $R \sim 12^{\prime \prime}$ (measured from the image). The final profile shows the prototypical Type II.o-CT behaviour. A sharp break at $\sim 85^{\prime \prime}$ followed by a downbending profile. The break is way to far out for a normal Type II.oOLR break given the small bar size, and corresponds roughly to the end of the visible spiral arm structure.
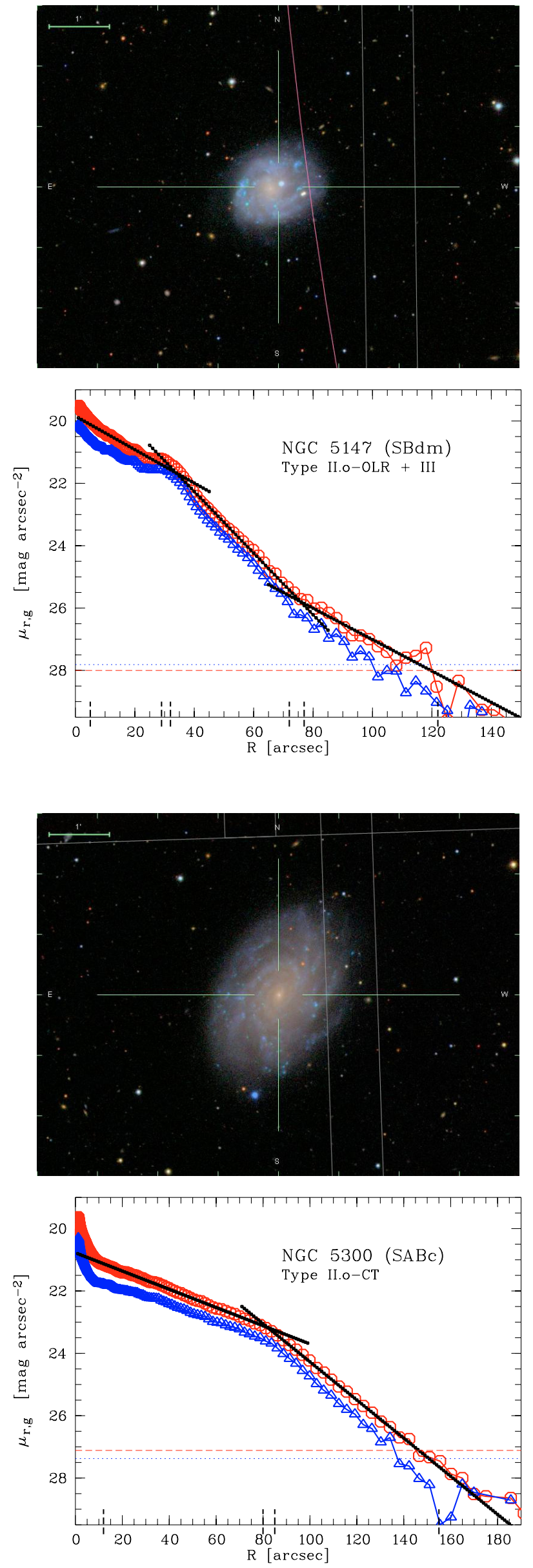
NGC 5334: Type II.o-CT

J135254.4-010651.SBT5* 5.0 -19.223 .91433$

Galaxy has a bar with a visible dust lane of size $R \lesssim 20^{\prime \prime}$ as measured from the image. The final profile is clearly downbending, but the exact position of the break is difficult to place. The extended break region resembles in this case again a straight line, so that one could also define two break radii at $\sim 85^{\prime \prime}$ and $\sim 125^{\prime \prime}$. However, since the first one is not even close to twice the bar radius and no other particular feature is responsible for the second break, the galaxy is classified Type II.o-CT with a single break at $\gtrsim 90^{\prime \prime}$.

NGC 5376: Type II.o-OLR

J135516.1+593024.SXR3\$ $3.0-20.002 .12293$

Galaxy close to the border of the SDSS field but almost complete. It has a known, similar-sized companion (UGC 08859 , $v=1610 \mathrm{~km} \mathrm{~s}^{-1}$ ) about 7.4' away and there is another possible, much fainter dwarf companion (3.5' away towards northwest) visible on the image. The final profile shows a weak break at $\sim 35^{\prime \prime}$ coinciding with the end of some very faint spiral arms. The dip at $\sim 14^{\prime \prime}$ and the peak at $\sim 18^{\prime \prime}$ are related to a pseudoring structure (mentioned also in RC1) around a possible bar. Although the exact size of the bar is difficult to fix, assuming it is there, the break is close to twice the inner ring radius and therefore classified as Type II.o-OLR.
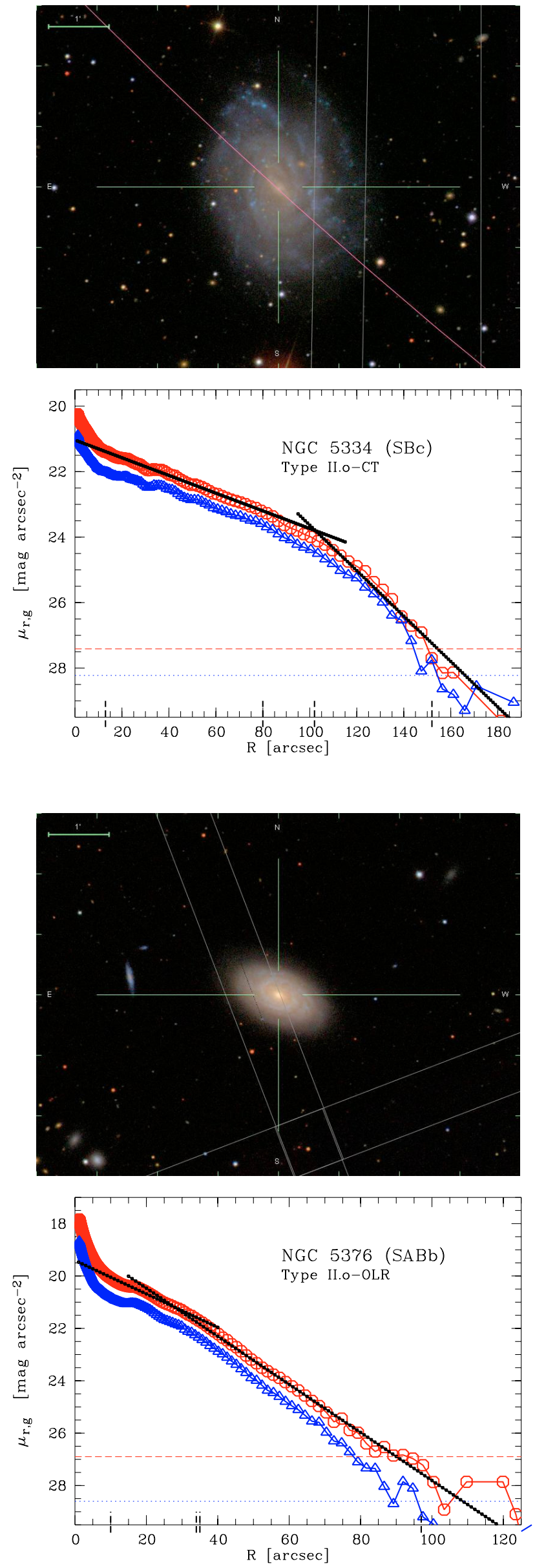
NGC 5430: Type II.o-OLR

J140045.8+591943.SBS3. $3.1-20.862 .2 \quad 3238$

Galaxy is isolated according to Prada et al. (2003). The center is dominated by a strong bar with dustlanes of size $R \sim 35^{\prime \prime}$, followed by two wrapped spiral arms. The very center is slightly elongated, looking twofold (effect of dust lane?), thus the centering is uncertain. The extended bump at $\sim 45^{\prime \prime}$ in the final profile is associated with the wrapped spiral arms. We have included the bump and bar for fitting the inner scalelength, because it does not change the result much. It is in principle possible to fit the full profile with a single exponential (Type I), allowing for this extended bump. However, we chose to fit a break at $\sim 70^{\prime \prime}$. This reduces the deviation from the model and since it is about twice the bar radius the galaxy is classified as Type II.o-OLR.

NGC 5480: Type III-d

J140621.5+504332 .SAS5* $5.0-19.721 .72119$

A large scale gradient (from top to bottom) in the background of the $r^{\prime}$ band image is removed with a linear fit. Galaxy forms a close pair with NGC $5481\left(v=2143 \mathrm{~km} \mathrm{~s}^{-1}\right)$ only $3.1^{\prime}$ away with the very outer isophotes starting to overlap. Galaxy appears to consist of two regions. An inner disk with the bright center and the spiral arms of size $R \sim 40^{\prime \prime}$ with a common ellipticity and PA, sitting in a more diffuse outer disk having a different ellipticity and a nearly orthogonal PA. The systems looks like an extended bar in an underlying disk, similar to NGC 1068 and the photometric inclination (ellipticity) and PA is difficult to determine and therefore uncertain. The final profile exhibits a break with an upbending profile at $R \sim 75^{\prime \prime}$, thus classified as Type III, which is consistent with the profile shown by Courteau (1996) (cf. UGC 09026). Between the inner disk and the break there is indication for two symmetric, thick spiral arms emerging from the inner bar-like disk.
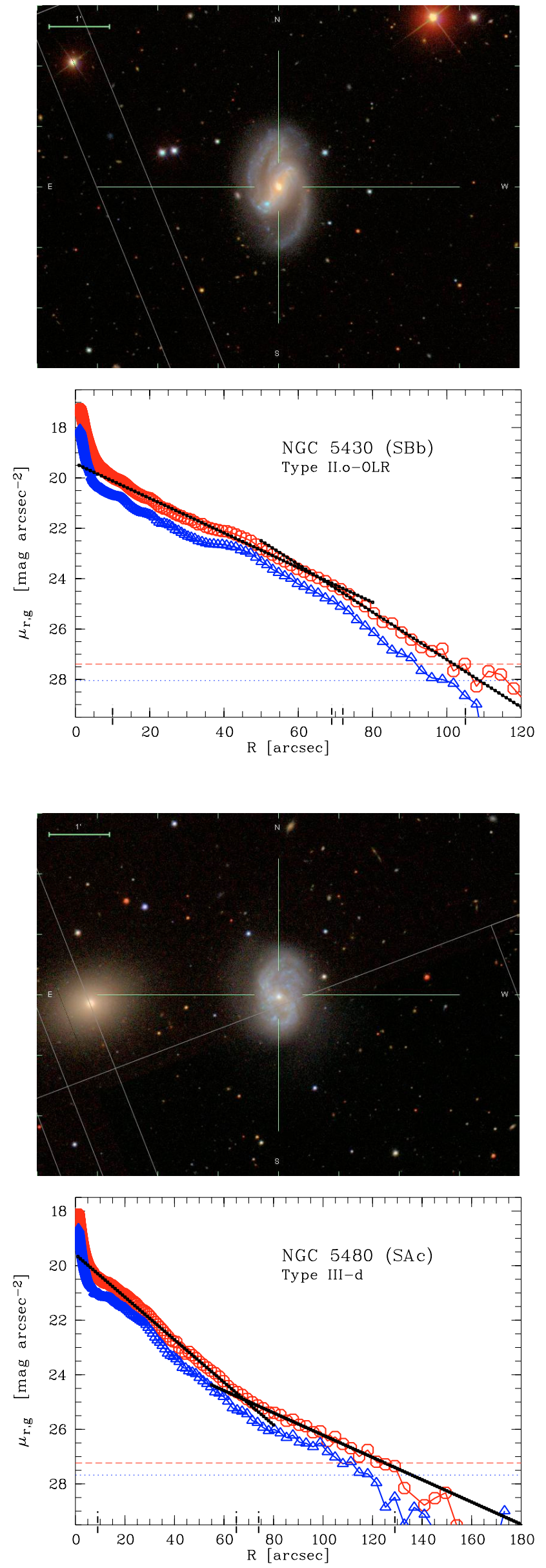
NGC 5584: Type II.o-CT

J142223.8-002315.SXT6. $6.1 \quad-19.80 \quad 3.21702$

Bright foreground star nearby but the influence is small. Two additional brighter stars in the outer disk force extended masking. The inner disk is dominated by two prominent spiral arms plus some more asymmetric ones with many starforming patches. The galaxy is classified as $\mathrm{SAB}$, but the bar size $\left(R \sim 10^{\prime \prime}\right)$ is difficult to determine, since the spiral arms seem to continue towards the center. Similar to NGC 3488 the profile shows a clear downbending, however, with uncertain break radius due to the extended break region which resembles in this case again a nearly straight line, so that one could also define two break radii at $\sim 60^{\prime \prime}$ and $\sim 110^{\prime \prime}$. Since the bar is too small to be responsible for the inner break we use a single break at $\sim 90^{\prime \prime}$ enclosing roughly the inner spiral structure followed by a slightly asymmetric outer disk (one side with a clearly more diffuse appearance).

NGC 5624: Type III

J142635.3+513503 .S? .. $5.0-18.691 .12186$

Large scale gradient (from top to bottom) in the background of the $r^{\prime}$ band image is removed with a linear fit. Very small galaxy which is most probably of noticeably later type than classified (Sc). Inner region dominated by an offcenterd (compared to the outer isophote used for centering) narrow bar, or rim-like structure of size $\sim 10^{\prime \prime}$ which is responsible for the central dip in the profile. The PA of the outermost region is twisted compared to the inner, bar-like structure. The final profile exhibits a straight line starting from $\sim 20^{\prime \prime}$ (enclosing the central region including the bar having the same PA) down to a break at $\sim 45$ followed by an upbending profile (classified as Type III) into the more symmetric, diffuse outer disk without visible substructure.
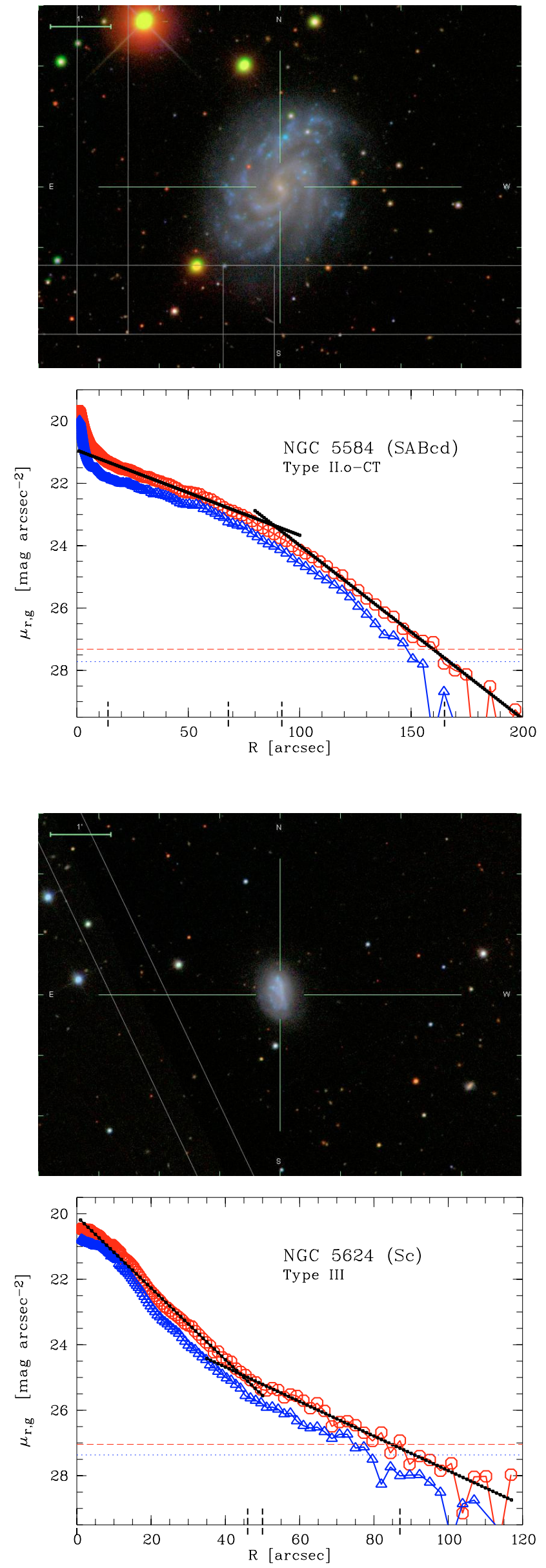
NGC 5660: Type II-CT

J142949.8+493722 .SXT5. $5.1 \quad-20.62 \quad 2.8 \quad 2585$

Galaxy close to the edge of the SDSS field but almost complete sitting in a strip with higher background. According to Sandage \& Bedke (1994) it has a small companion nearby ("a likely dwarf Im companion of unknown redshift"), however this could be associated with UGC $09325\left(v=3730 \mathrm{~km} \mathrm{~s}^{-1}\right)$ being clearly a background galaxy. Galaxy shows two, slightly asymmetric inner spiral arms plus an additional one extending towards the outer disk. Although classified as SAB there is no bar-like structure visible except for a possible ring-like structure of size $R \sim 5^{\prime \prime}$. The final profile is not well described with a single exponential (Type I), already excluding the region beyond $R \sim 90^{\prime \prime}$ which is most probably affected by an incorrect sky subtraction. Unaffected of this it is better described as having a break at $\sim 70^{\prime \prime}$, corresponding to the end of the single extended spiral arm, with a following downbending profile, which is too far out to be related to the possible bar and therefore classified as Type II-CT. The bump at $\sim 40 "$ is related to the end of the inner spiral arms and beginning of the outer extended one. NIR imaging of the underlying old disk population should give clarification to the classification.
NGC 5667: Type II.i + III

J143022.9+592811 .S. .6* $5.8-19.691 .72222$

Galaxy small and close to the edge of the SDSS field but almost complete with an untypical appearance. The inner region $\left(R \lesssim 30^{\prime \prime}\right)$ looks like an extended bar with dustlanes and a possible additional, tilted, secondary inner bar of size $R \lesssim 7^{\prime \prime}$. Outside this region there are two asymmetric narrow spiral arms visible towards north-west followed by an outer, slightly asymmetric, envelope, which makes the photometric inclination (ellipticity) and PA uncertain. The final profile shows a downbending break at $\sim 25^{\prime \prime}$, related to (and inside) the extended bar structure, therefore classified as Type II.i, followed by an exponential down to another break at $\sim 65^{\prime \prime}$ where an additional upbending starts adding the Type III classification. The outer profile is uncertain due to a higher error in the sky. A still justifiable sky value could create a continuous outer slope, but this is unlikely, since it will not create the asymmetric shape of the outer isophotes.
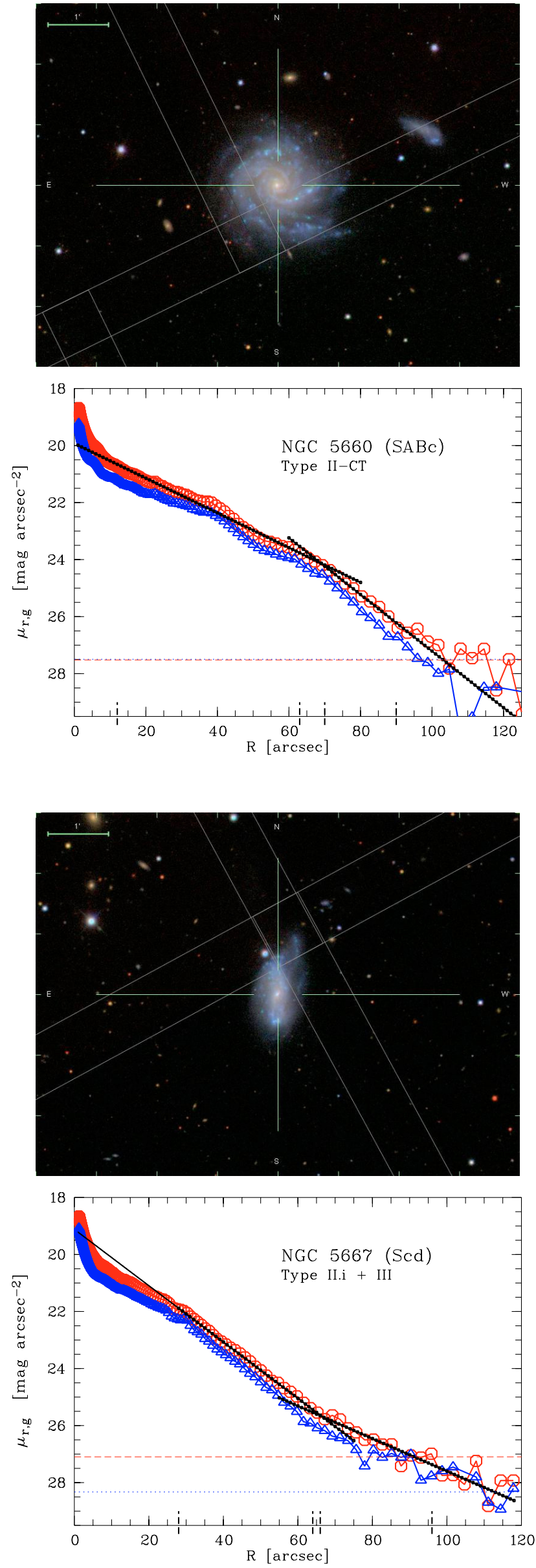
NGC 5668: Type I

J143324.4+042702 .SAS7. $6.7 \quad-19.65 \quad 2.81672$

Galaxy close to the edge of the SDSS field but almost complete. Although classified as SA there is a weak bar or oval inner structure of size $R \sim 12^{\prime \prime}$ visible on the image, reflected by a small shoulder in the final profile. The outer disk (beyond $R \sim 100^{\prime \prime}$ ) is slightly asymmetric and more extended towards the North. The bump at $\sim 70^{\prime \prime}$ in the final profile is related to brighter spiral arms in this region. There is a possible upbending break visible at $\sim 100^{\prime \prime}$ (associated to the asymmetric outer disk) or $\sim 140^{\prime \prime}$ but the scalelength contrast is too low and it is too close to the sky error to argue for a Type III classification.

NGC 5693: Type II-AB

J143611.2+483504.SBT7. 6.8 -18.64 1.92537

Small galaxy with unusual appearance, showing an inner extended bar-like structure of size $R \sim 15^{\prime \prime}$, apparently with two central peaks, probably caused by a superimposed foreground star. The brightest pixel in the bar structure does not correspond to the center obtained from the outer isophotes and is off by $\sim 7^{\prime \prime}$ causing the inner dip in the final profile. The bar-like structure is followed by a single spiral arm extending towards the outer (quite roundish and symmetric) disk, which is used to obtain the ellipticity and PA. The final profile cannot be well fitted with a single exponential (Type I) but rather shows a downbending behaviour albeit with an uncertain break radius due to the extended break region which resembles again a nearly straight line, so that one could also define two break radii at $\sim 30^{\prime \prime}$ and $\sim 50^{\prime \prime}$. Although the first break is at twice the bar radius, we classify this galaxy as Type II-AB since this whole region is clearly lopsided with an significantly offcentered bar.
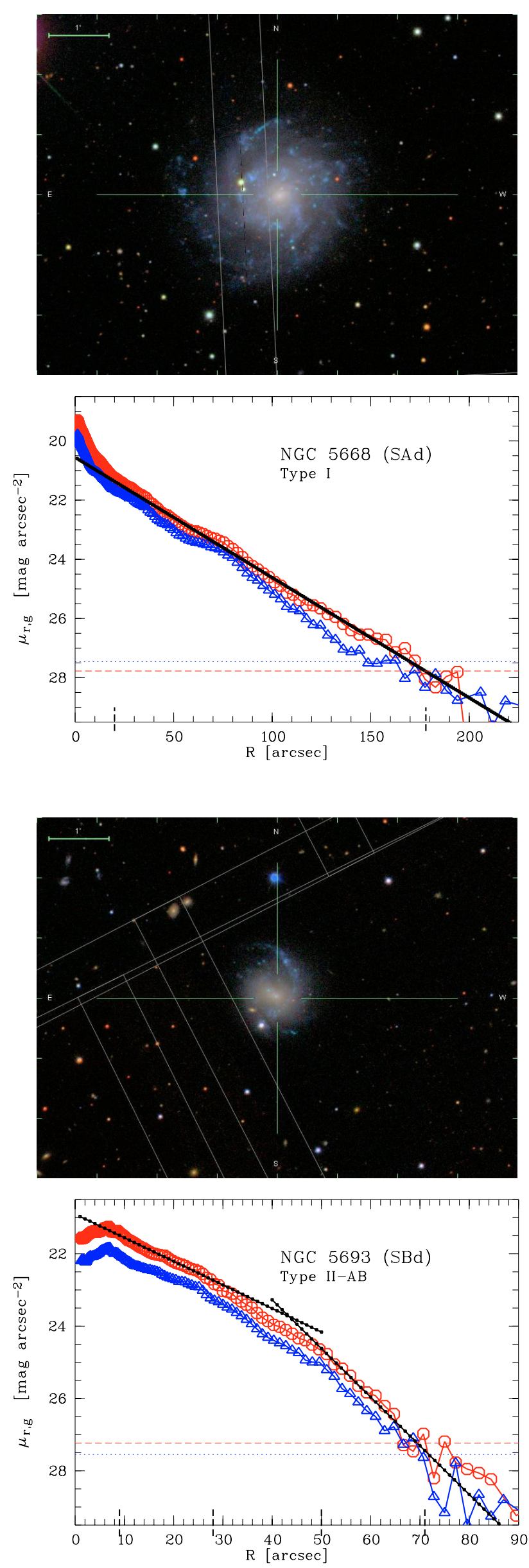
NGC 5713: Type III

J144011.4-001725.SXT4P $4.1 \quad-20.502 .8 \quad 1958$

According to Sandage \& Bedke (1994) in a group of latetype spirals with four members in a region smaller than the Local Group. No bright nucleus in the bar-like structure so the centering is done from the outer isophotes. In addition to a more chaotic inner region with spiral structure (producing the bump between $R \sim 10-35^{\prime \prime}$ in the final profile) there is clear substructure in the outer disk visible. Towards the South $\left(1.5^{\prime}\right.$ from the center) we see a very diffuse additional patch of light (size $R \lesssim 20^{\prime \prime}$ and masked), in addition to a more elongated feature $\left(\approx 1^{\prime}\right.$ from the center, unmasked) towards north-east. The profile shows a break at $\sim 115^{\prime \prime}$ with an upbending profile associated with a rather symmetric featureless outer part. The break is not caused by a possible sky error thus classified as Type III.

NGC 5768: Type I

J145207.9-023147.SAT5* 5.0 -19.241 .62018$

Bright star superimposed so using an extended mask. Photometric inclination (ellipticity) at $1 \sigma$ in the free ellipse fit does not match the outer parts well, therefore we used the outermost ellipse which leaves the ellipticity and PA uncertain. Similar to NGC 3888 the final profile exhibits an extended wiggle (with an unusual integral sign shape) at $\sim 50^{\prime \prime}$ (without being a break) corresponding to a set of spiral arms in the outer disk which do not start at the center. The inner break at $\sim 18^{\prime \prime}$ is associated with the inner spiral arms and the barlike center. Although showing extended wiggles the galaxy is classified as Type I since there is no better fitting alternative.
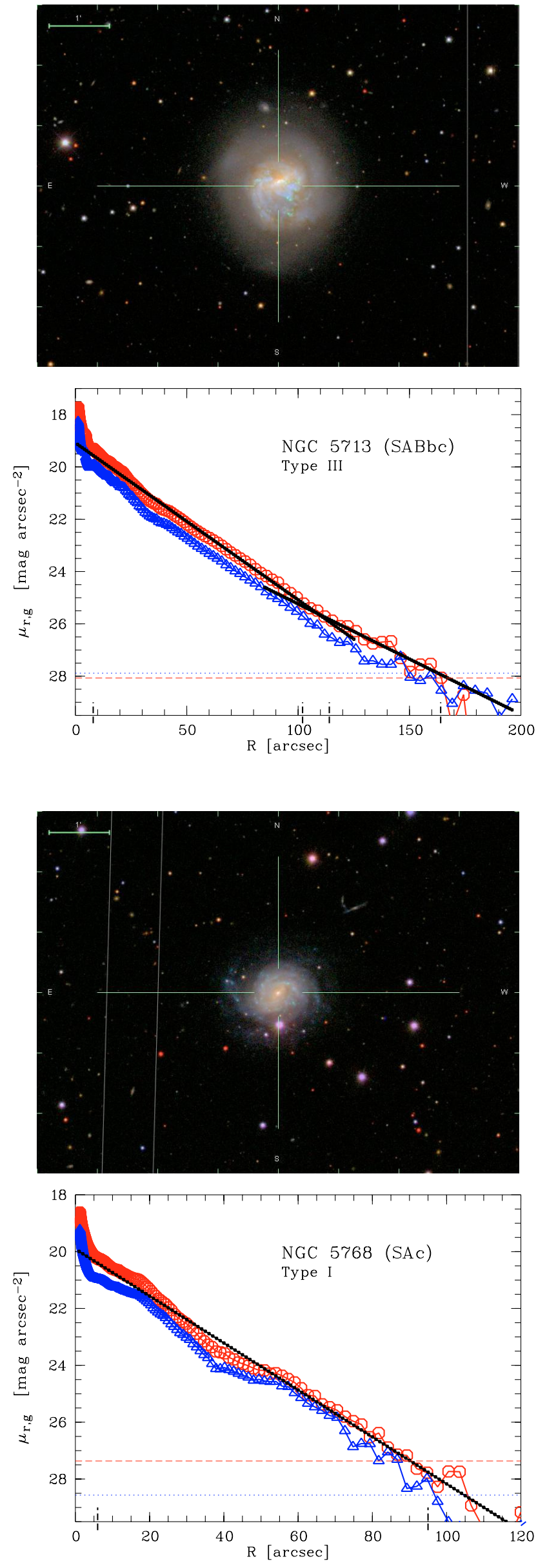
NGC 5774: Type II.o-OLR + III

J145342.6+033500.SXT7. $6.9-19.092 .81655$

Galaxy has a close physical, edge-on, companion (NGC 5775, $\mathrm{SBc}$ ?, $v=1681 \mathrm{~km} \mathrm{~s}^{-1}$ ) and exhibits an outer, extended spiral arm pointing towards NGC 5775 (similar to the M 51/NGC 5159 system but having a more similar sized mass) producing an asymmetric outer disk where the very outer isophotes already overlap. The center is obtained from outer ellipse fits (different from brightest pixel) since the inner elongated, narrow, bar-like region $R \sim 10^{\prime \prime}$ is without an obvious nucleus. The shoulder in the final profile at $\gtrsim 35^{\prime \prime}$ corresponds to the end of the more extended bar-like structure enclosing the inner bar having a different PA. The break at $\sim 80 "$ with the downbending profile being at about twice the extended bar radius is therefore classified Type II.o-OLR. The upbending profile (classified as Type III) beyond a break at $\sim 120 "$ is due to the asymmetric outer disk with extended spiral arms. Fitting the profile with a single exponential (Type I) leaves three extended wiggles which are better explained by a Type II.o-OLR + III break combination.

\section{NGC 5806: Type III}

J150000.4+015329.SXS3. $3.3-19.743 .01440$

Galaxy close to our high axis ratio limit (dust lane visible?) and close to the edge of the SDSS field but almost complete. From the inner disk there is an additional patch of diffuse light visible towards the south-east (not masked) extending into the asymmetric outer disk, which makes the photometric inclination (ellipticity) and PA uncertain since obtained further in. The final profile shows a shoulder at $\sim 40^{\prime \prime}$ related to the inner bar-like center with the beginning of the spiral arms and a break at $\sim 120^{\prime \prime}$. The break is close to the position of the extra patch of light followed by an upbending profile (classified as Type III) extending into the outer asymmetric disk without spiral structure. Our classification agrees well with Erwin et al. (2006) and the Type III profile is also visible in Courteau (1996) (cf. UGC 09645).
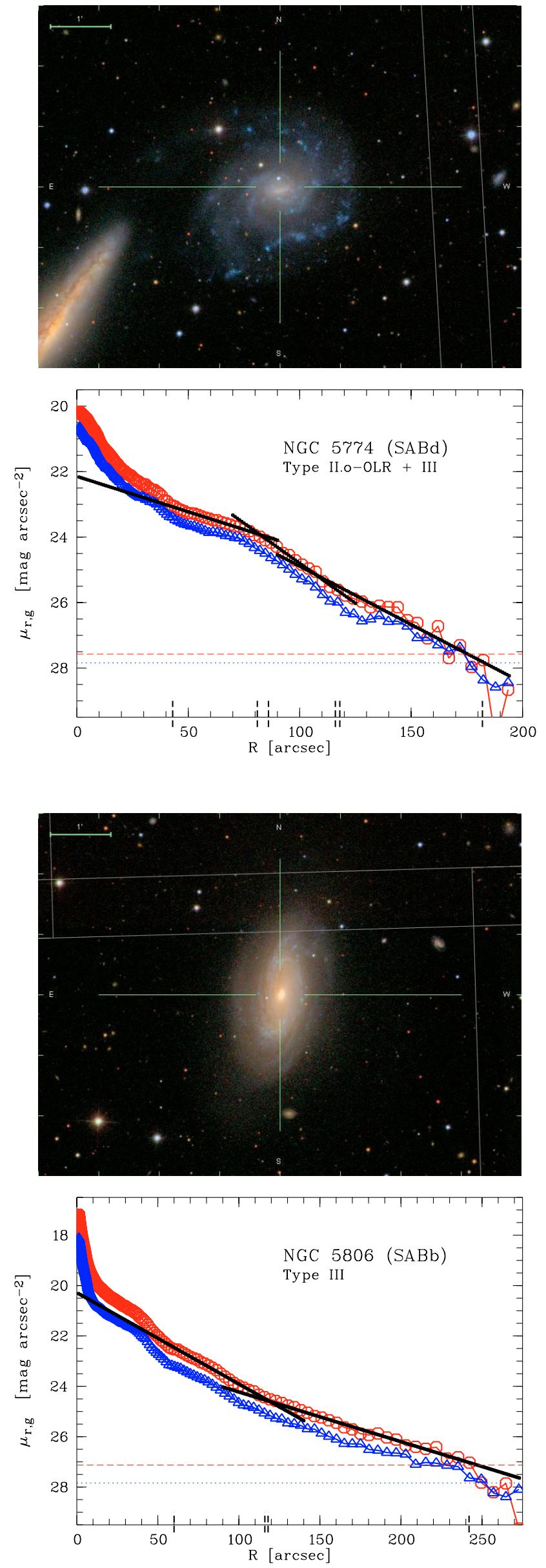
NGC 5850: Type II.o-OLR

J150707.7+013240.SBR3. $3.0-21.434 .32637$

Bright star nearby needs extended mask. The $r^{\prime}$ band background is slightly inhomogeneous. Galaxy is dominated by an extended bar of size $R \sim 55^{\prime \prime}$ enclosed by an inner ring out to $R \sim 75^{\prime \prime}$ having a secondary, inner bar of size $R \sim 7^{\prime \prime}$ with clearly different ellipticity and PA. There is an additional, faint outer ring (or pseudo-ring, since looking similar to some wrapped spiral arms) visible on the image related to the break at $\sim 130^{\prime \prime}$ in the final profile, thus obviously classified as Type II.o-OLR. Since the outer ring is used to determine the photometric inclination (ellipticity) and PA they are rather uncertain.
NGC 5937: Type III

J153046.1-024946 PSXT3P $3.2 \quad-20.901 .92870$

Bright stars nearby need extended mask without really covering the outer disk. There is a possible, small dwarf companion (not in NED) visible about $1.5^{\prime}$ away towards the northwest. The outer disk is slightly asymmetric with continuously changing ellipticity and PA, so the values applied for the final profile are rather uncertain. The break at $\sim 70^{\prime \prime}$ with an upbending profile (classified as Type III) is most probably related to the changing ellipticity and should be taken with caution.
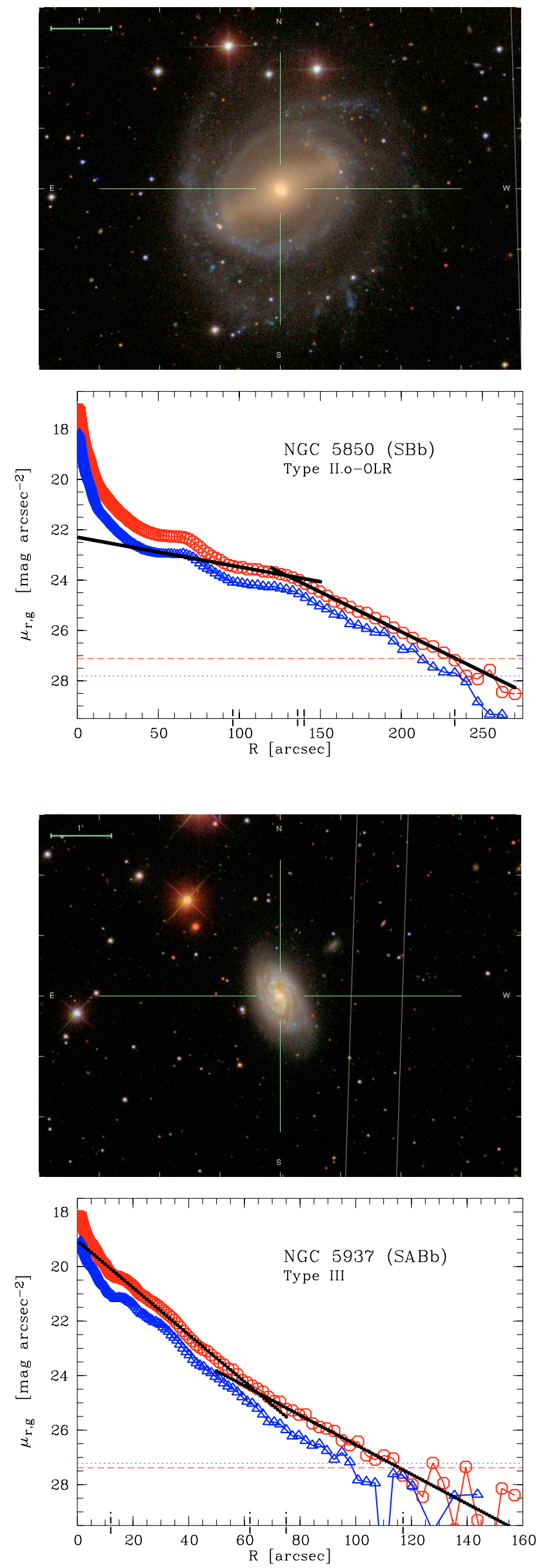
NGC 6070: Type II.o-CT

J160958.9+004234.SAS6. $6.0-21.04 \quad 3.42085$

Galaxy close to our high axis ratio limit having a very bright star (with an extended halo) in the FOV which is still small enough to avoid significant influence. Only partly fitted since $\lesssim 1 / 5$ of galaxy is beyond SDSS field. The outer isophotes are slightly asymmetric, with one sharp and one more fluffy side, so the ellipticity and PA are uncertain. The final profile exhibits a clear downbending with a more extended break region around $R \sim 95$ " corresponding to the end of the spiral arm structure and classified as Type II.o-CT. Although the galaxy is classified as SA there is an elliptical central bar-like structure of size $\$ 10^{\prime \prime}$ visible which would be however too small to argue for a Type II.o-OLR break. The small dip at $\sim 30^{\prime \prime}$ is produced by spiral arm contrast.

NGC 6155: Type II-AB

J162608.3+482201 .S?... 7.8 -19.45 1.32684

Small galaxy with an extended mask reaching into the outer disk to cover a very bright star close by. Lopsided disk with centering done from the outer isophotes. The center is $\sim 3$ " off the brightest pixel associated to a small bar-like central structure of size $R \sim 5^{\prime \prime}$ followed by a thin, star-forming, blue spiral arm feature towards north-east. The final profile shows a downbending break at $\sim 35^{\prime \prime}$ corresponding to the inner lopsided disk thus classified as a Type II-AB break, although this is just at the radius of an outer ring (best visible in the colour image).
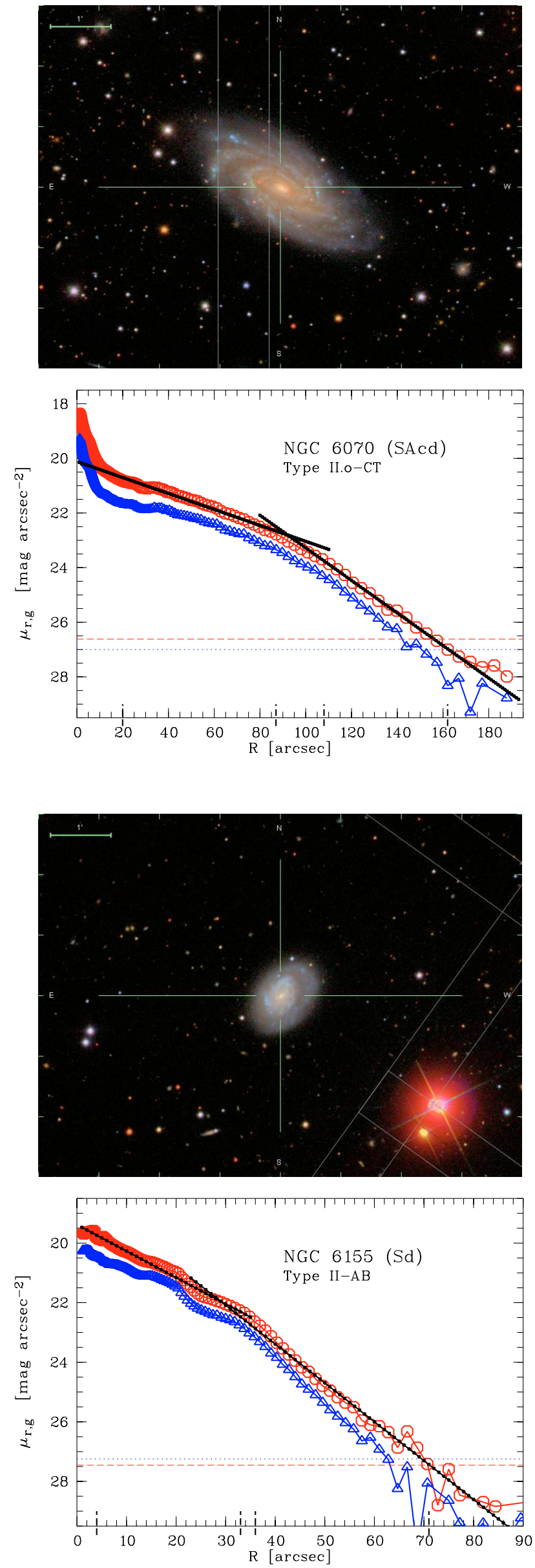
NGC 7437: Type II-CT

J225810.0+141832 .SXT7. $7.1 \quad-18.88 \quad 1.82190$

Small galaxy close to the edge of the SDSS field but almost complete. Similar to NGC 5300 the final profile shows the prototypical Type II.o-CT behaviour. A sharp break at $\sim 40$ ", corresponding roughly to the end of the apparent flocculent spiral arm structure, followed by a downbending profile. Although classified as SAB there is no obvious structure visible which could be identified with a bar. Using the beginning spiral arms as an upper limit $\left(R \lesssim 8^{\prime \prime}\right)$ the break is still too far out for a normal Type II.o-OLR break.

\section{NGC 7606: Type II-CT}

J231904.8-082906 .SAS3. $3.2 \quad-21.384 .32187$

Galaxy with multiple thin spiral arms. The final profile exhibits clearly a break at $\sim 110^{\prime \prime}$ corresponding roughly to the end of the visible spiral arm structure, following part of the ring enclosing an apparent hole (enhanced dust extinction?) in the disk towards the north-west. Since the galaxy is classified as SA and no bar structure visible the break is a Type II-CT. The apparent upbending of the profile beyond 190" is not related to a sky error but due to a changing ellipticity in the very outer disk (maybe due to a warp?).
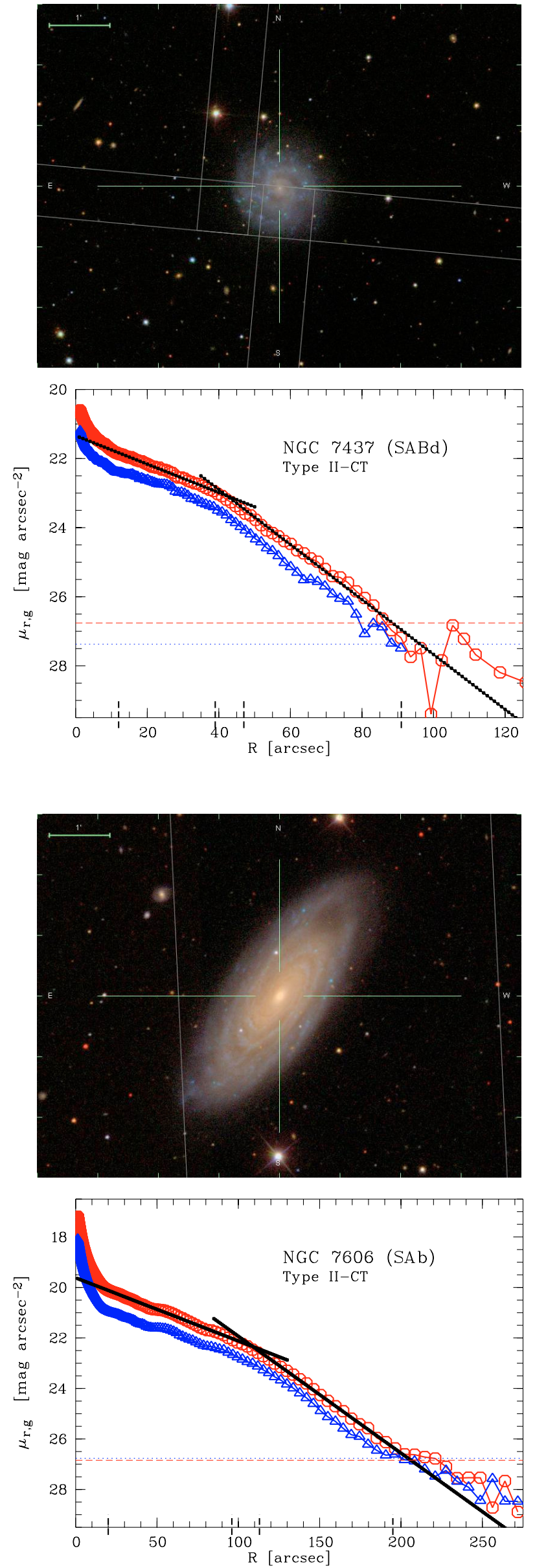
PGC 006667 $\equiv$ UGCA 021: Type II-AB

J014910.2-100345 .SBS7. $7.1-18.772 .91887$

A large scale gradient (from top to bottom) in the background of the $r^{\prime}$ band image is removed with linear fit. Galaxy in NGC 701 group (see above). Final profile shows a bump at $\sim 20^{\prime \prime}$ due to the spiral arms starting from the bar-like center. The clear downbending break at $\sim 75^{\prime \prime}$ corresponds to a transition from the inner more symmetric to an asymmetric, extended outer disk region and is therefore classified as Type IIAB.

UGC 02081: Type II-CT

J023600.9+002512 .SXS6. $5.8-18.561 .82549$

Galaxy close to the edge of the SDSS field but almost complete. The final profile shows a downbending break starting around $\sim 55^{\prime \prime}$ (clearly at $\sim 70^{\prime \prime}$ ) with a very faint, but symmetric light distribution beyond. Although classified as SAB the bar is not obvious from the image but the spiral arms allow to estimate an upper limit of $R \lesssim 7^{\prime \prime}$. Therefore the break (which is not produced by a sky error) is classified Type II-CT. The profile published in de Jong \& van der Kruit (1994) is not deep enough to confirm the downbending.
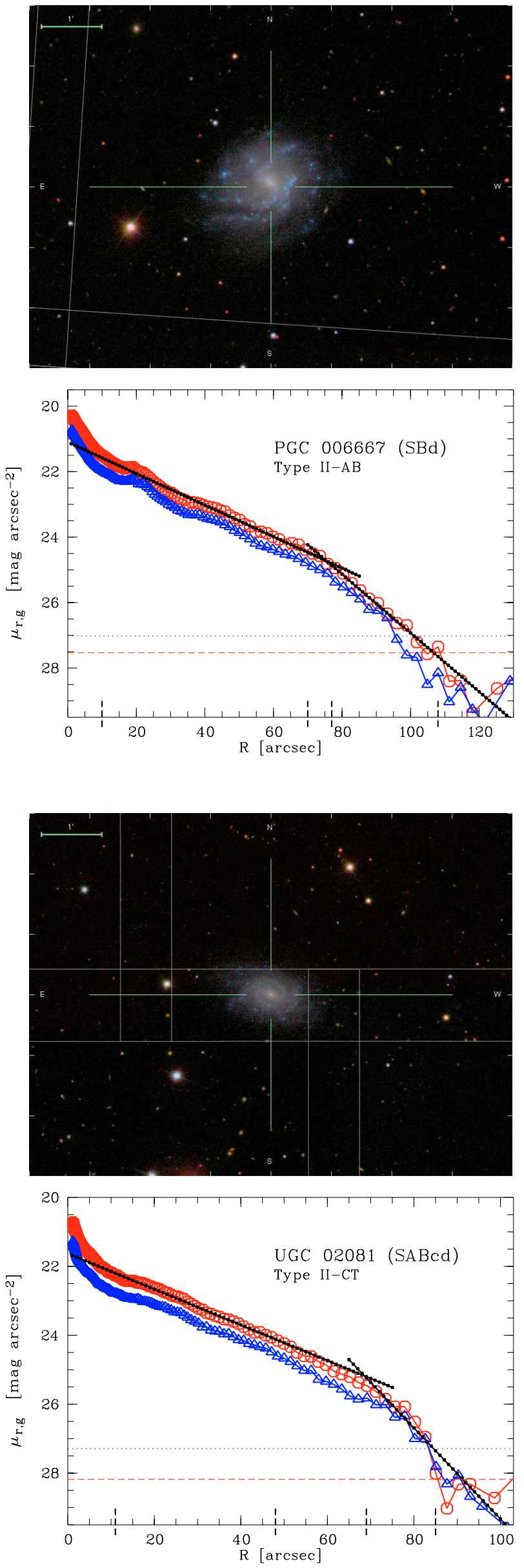
UGC 04393: Type I

J082604.3+455803 .SB?.. $5.5 \quad-19.492 .22290$

Galaxy is classified as SB and the inner region is dominated by an elongated bar-like structure of size $R \sim 30^{\prime \prime}$. Therefore this region is excluded from the fit. The extended bar, having a kind of double nucleus, is slightly offcenter (central dip in profile) compared to the center obtained from the outer isophotes and is followed by a single, narrow spiral arm like structure extending south-west. In the outer disk there is an additional, faint spiral arm visible towards the south-east. There is also a very faint shell or stream-like structure detected, detached from the galaxy about $2^{\prime}$ away to the north-west. The inclination (ellipticity) and PA are rather uncertain since they are continuously changing towards the outer disk. The final profile, beyond the inner bar region, is classified as Type I, due to the uncertain ellipticity and the asymmetric outer disk with the spiral arm, although there are two possible breaks visible at $\sim 60^{\prime \prime}$ and $\sim 80^{\prime \prime}$, of which the first could correspond to a Type II.o-OLR break and the latter to a Type III.

\section{UGC 06309: Type II.i}

J111746.4+512836 .SB? .. $5.0-19.631 .33097$

There is a small BCG (MRK 1445) about 3.5' away at roughly the same distance $\left(v=2863 \mathrm{~km} \mathrm{~s}^{-1}\right)$. Galaxy exhibits very unusual, disturbed shape. The inner disk is dominated by a narrow bar of size $R \lesssim 20^{\prime \prime}$ followed by two asymmetric spiral arms (one highly wrapped, forming an inner ring, the other almost extending straight). The disk beyond the spiral arms is also highly asymmetric and more egg-shaped with a region of low emission (high extinction?) towards the south-east, which makes the bar appear to be offcentered. However, the center from the very outer isophotes coincides again with the nucleus inside the bar. The extended dip and peak structure around $15-20^{\prime \prime}$ in the final profile corresponds to the inter-arm region, respectively the pseudoring build by the arms, so the break around this radius is classified as Type II.i. This should be used with caution, since alternatively one can argue for a break at $\sim 35^{\prime \prime}$, at about twice the bar radius thus a Type II.o-OLR break, while excluding the region between 15-30".
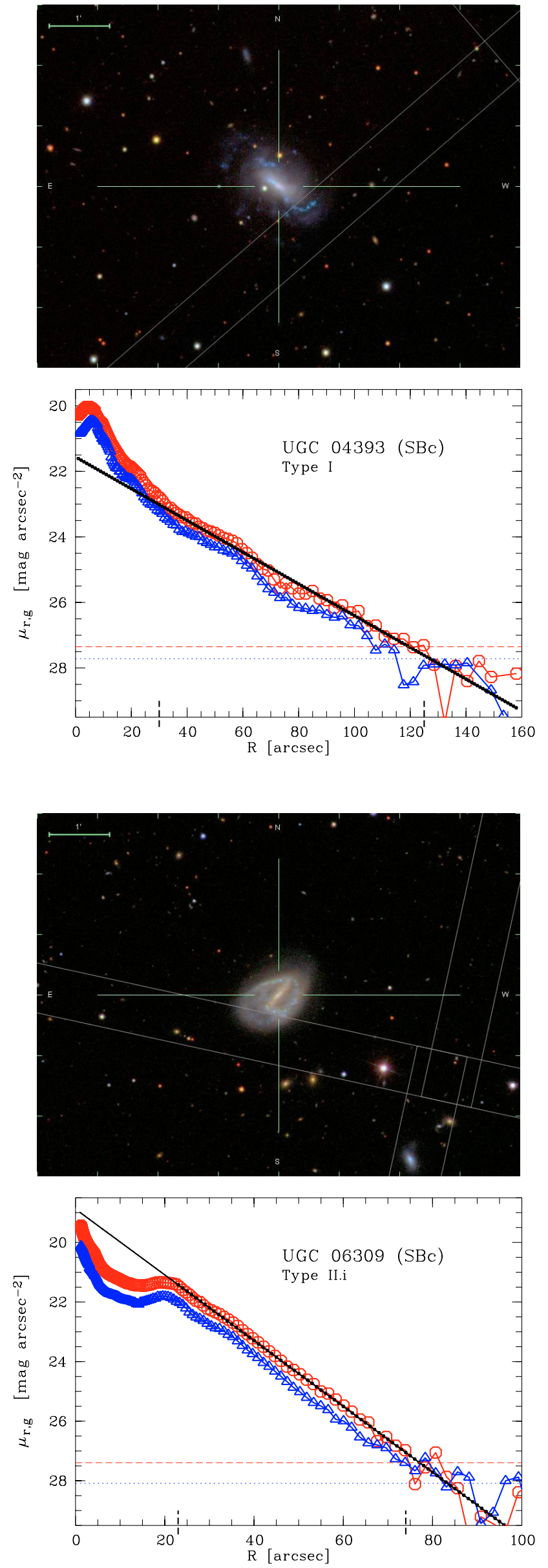
UGC 06518: Type II.i

J113220.4+535417

\section{$4.3-18.94 \quad 1.0 \quad 3044$}

Small galaxy where, although not classified as barred in NED or LEDA, there is clearly a bar of size $R \sim 10^{\prime \prime}$ visible, with indication for a ring-like structure around, already reported by Takase \& Miyauchi-Isobe (1984). Despite the possibility to argue for a break at $\sim 15^{\prime \prime}$ in the final profile we classify the galaxy as Type II.i excluding the region inside $R=10^{\prime \prime}$ from the fit.

\section{UGC 06903: Type II.o-CT}

J115536.9+011415 .SBS6. $5.8-18.802 .51916$

Very bright star in FOV but galaxy not influenced. Final profile shows clearly a downbending break around $\sim 65^{\prime \prime}$ corresponding roughly to the end of the visible spiral arm structure and classified Type II.o-CT, since the size of the bar with $R \lesssim 17$ is too small. The extended bump between $R \sim 20-40^{\prime \prime}$ is related to the inner two, highly wrapped (pseudoring?) spiral arms. The downbending shape is consistent with the profile shown in Jansen et al. (2000) (cf. ID 99).
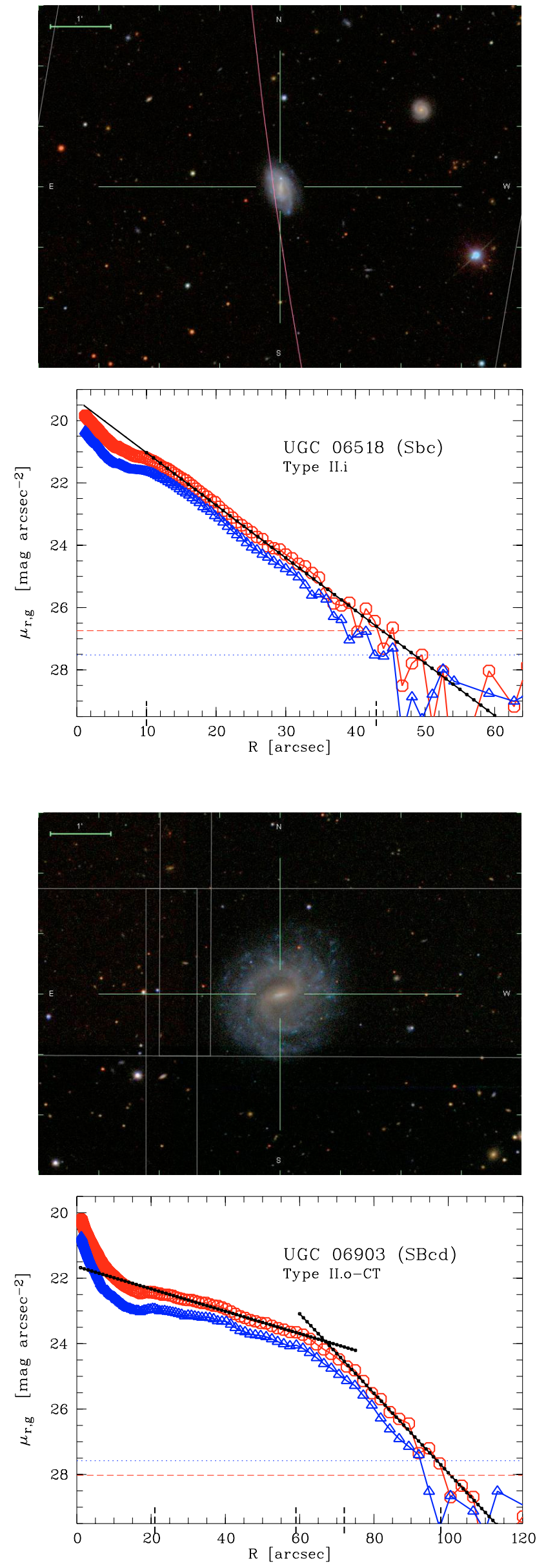
UGC 07700: Type II.o-CT

J123232.8+635238 .SBS8. $7.8 \quad-18.501 .8 \quad 3239$

There is a very bright star in the FOV but the galaxy is not influenced. On same image there is an edge-on companion (S0/a galaxy NGC 4512) at similar distance $\left(v=2536 \mathrm{~km} \mathrm{~s}^{-1}\right)$ visible. Ellipticity obtained from the very outer isophotes and not by the usual $1 \sigma$ criterion. The final profile shows clearly a downbending behaviour outside the bar region. The bar is about $R \sim 17^{\prime \prime}$ in size and slightly offcentered (by $\sim 5^{\prime \prime}$ ) compared to the center obtained from the outer, symmetric isophotes. This causes the dip at the center. The extended break region is not exponential but shows a curvature, but still one could define two break radii at $\sim 40^{\prime \prime}$ and $\sim 50^{\prime \prime}$. The region in between corresponds to a single spiral arm extending towards the north-east. Although the first one would roughly classify as a Type II.o-OLR break we used a single break slightly further out and classified the galaxy as Type II.o-CT.

UGC 08041: Type II.o-CT

J125512.7+000700 .SBS7. $6.8-18.62 \quad 3.21376$

Galaxy close to our high axis ratio limit and not influenced by the very bright star in FOV. The inner disk with a bright nucleus in the bar and weak spiral arm structure extends to about $\sim 100^{\prime \prime}$, followed by a rather asymmetric, lopsided outer disk extending to the south-west. Centering on the outer isophotes $\left(R \gtrsim 100^{\prime \prime}\right.$ ) would be off by more than $\sim 10^{\prime \prime}$. The final profile shows a downbending break at $\sim 75^{\prime \prime}$ roughly corresponding to the end of the spiral structure. Since the bar is only of size $R \lesssim 12^{\prime \prime}$ the break, at significantly more than twice the bar radius, is classified as Type II.o-CT. The fixed ellipse leaves the inner symmetric disk at about $\sim 100^{\prime \prime}$ into the asymmetric outer part causing the apparent upbending in the profile, which probably increases the fitted outer scalelength.
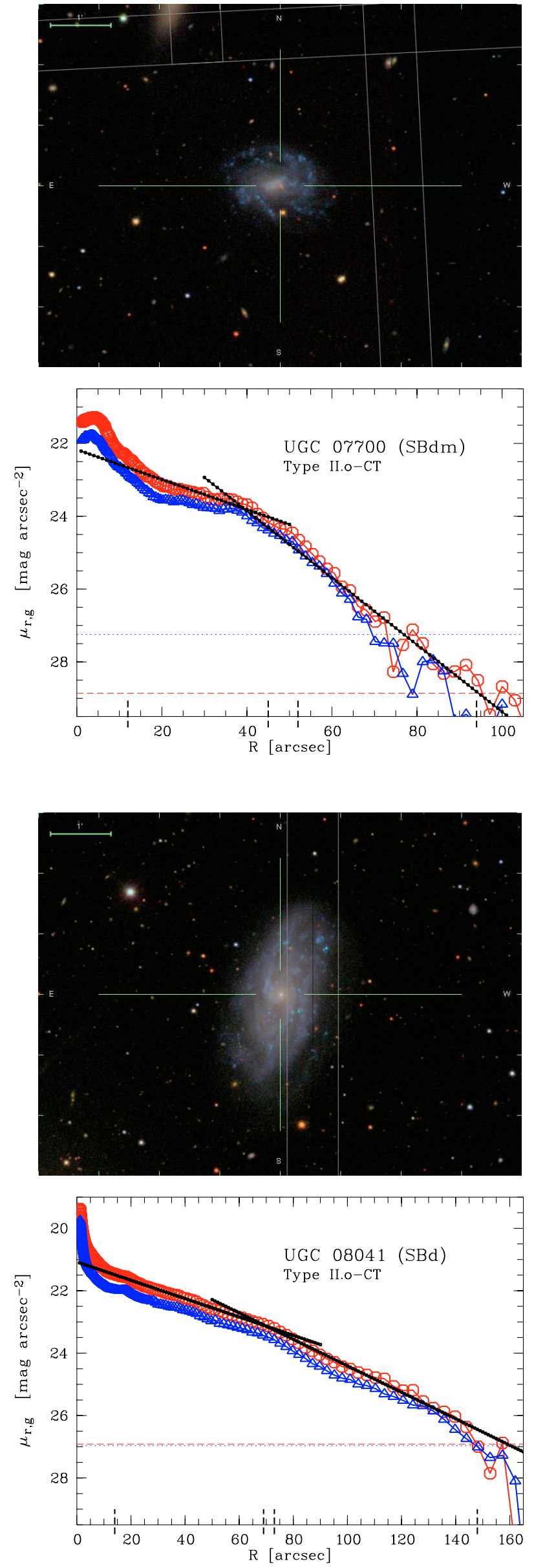
UGC 08084: Type II.o-OLR

J125822.4+024733 .SBS8. $8.1-18.61 \quad 1.32824$

Small galaxy dominated by an inner offcentered, asymmetric bar of size $R \sim 17^{\prime \prime}$, which appears to be z-shaped together with the two spiral arms. The center is obtained from outer isophotes $\left(\sim 50^{\prime \prime}\right)$ and is about $\sim 12^{\prime \prime}$ away from the brightest pixel in bar, which is responsible for the flat inner profile. The final profile shows clearly a downbending break at $\sim 40 "$ corresponding to the spiral arms forming a kind of pseudoring in the outer parts. Since the break is at about twice the bar size the galaxy is classified as Type II.o-OLR.

UGC 08237: Type II.o-OLR

J130854.5+621823 PSB. $3 * 3.2-19.610 .93120$

Galaxy is isolated according to Prada et al. (2003). The similar sized companion, only $2.3^{\prime}$ away, is a confirmed background galaxy (UGC 08234, $v=8100 \mathrm{~km} \mathrm{~s}^{-1}$ ). Background needs extended mask due to straylight from a very bright star off the field. UGC 08237 is a small galaxy dominated by a bright, thick bar of size $R \lesssim 12^{\prime \prime}$ (producing the shoulder in the final profile) followed by two nearly symmetric, wrapped spiral arms forming a pseudoring peaking at $\sim 20 "$. Similar to NGC 5701 (an early-type SB0/a galaxy from Erwin et al. (2006)) one could define a break at $\sim 25^{\prime \prime}$ just outside of the pseudoring forming the region $R \sim 12-25$ which is no longer well fitted by an exponential profile. Erwin et al. (2006) call these systems "extreme" OLR breaks, so we also classify the galaxy as Type II.o-OLR and apply a crude fit to obtain an inner scalelength.
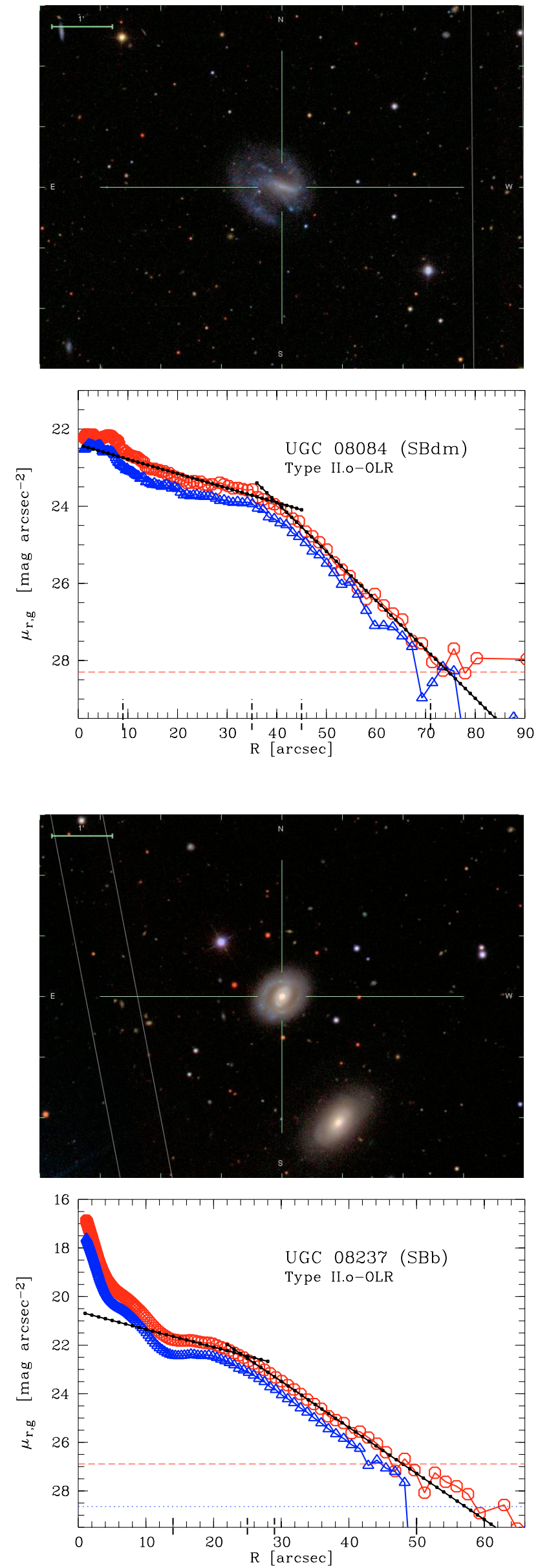
UGC $08658 \equiv$ Holmberg V: Type II-CT

J134039.9+541959.SXT5. 5.1 -19.912.6 2285

Galaxy close to the border of the SDSS field but almost complete. Although classified as SAB, the bar size is not obvious from the image, but could be restricted by the spiral arms to be maximal $R \lesssim 10^{\prime \prime}$. The shoulder in the final profile at $\sim 20^{\prime \prime}$ is due to the inner spiral arms being aligned with the ellipse. There is a weak break at $\sim 65^{\prime \prime}$ visible which roughly coincides with the end of the inner spiral arm structure, although there are maybe two faint extensions into the outer disk. Since being at significantly more than two bar radii we classify the break as Type II-CT.

UGC 09741 $\equiv$ NGC 5875A: Type III

J150833.5+521746 .S? . . $6.0-18.540 .92735$

The patch of faint light on the image towards the north-west is caused by straylight from a very bright star off field, but the galaxy is not affected. Very small galaxy dominated by a long, narrow bar of size $R \sim 9^{\prime \prime}$, enclosed by a ring like structure building the inner $R \lesssim 17^{\prime \prime}$ disk. The photometric inclination (ellipticity) and PA is obtained from the outer disk at $\sim 45^{\prime \prime}$ and not from the $1 \sigma$ ellipse. Although it is possible to fit the profile beyond $\sim 17^{\prime \prime}$ with a single exponential the offset inside would be too large to be assigned solely to a bulge component of a typical Sc galaxy. In addition, an extended bulge component is also not visible on the image inside the narrow bar. Thus we argue for a break, classified as Type III, at $25^{\prime \prime}$ followed by an upbending profile into the symmetric featureless outer disk. The shape of the profile is consistent with the one shown by Jansen et al. (2000) (cf. ID 166).
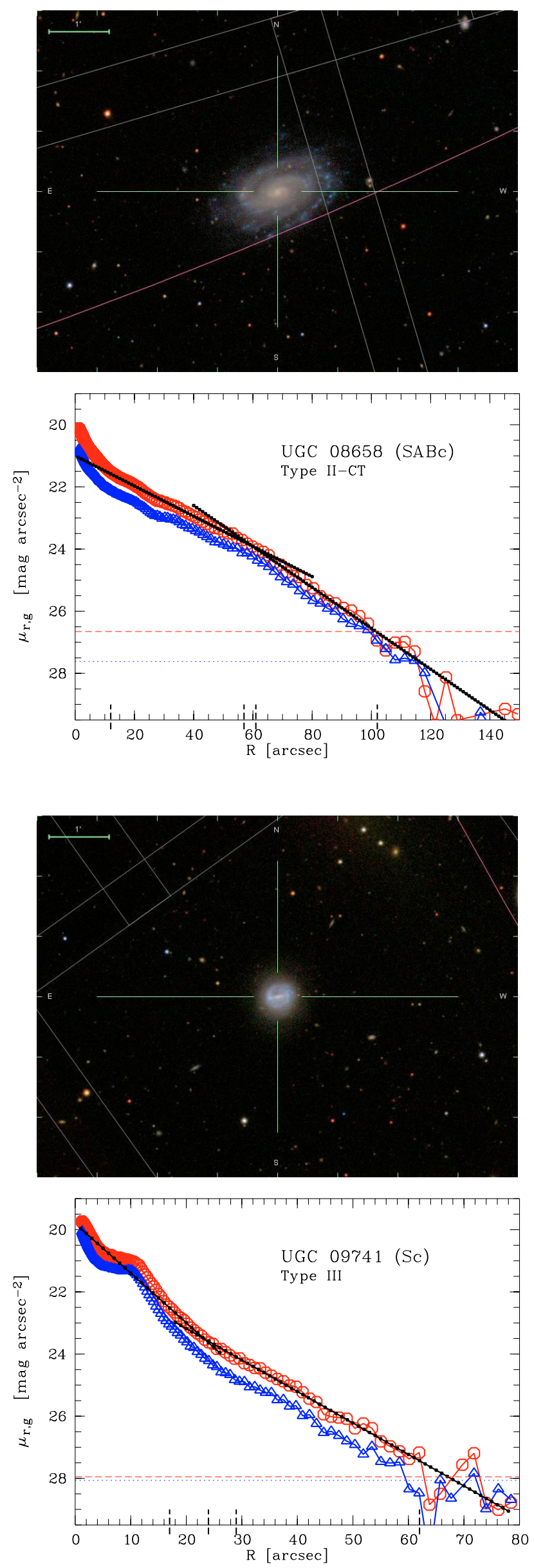
UGC 09837: Type II-CT

J152351.7+580311.SXS5. $5.1 \quad-19.45 \quad 1.82938$

Although classified as SAB, the bar size is not obvious from the image, but could be restricted to be maximal $R \lesssim 9^{\prime \prime}$ by the two symmetric spiral arms starting beyond the bulge/bar region out to $R \sim 25^{\prime \prime}$. Further out, there is more asymmetric spiral arm structure visible, with one arm almost forming a ring-like section, which makes the photometric inclination (ellipticity) and PA determination impossible at the $1 \sigma$ ellipse. Since the ring like section extends to the end of our free ellipse fit, we set the ellipticity to 0.05 (round). In the final profile there is a break visible at $\sim 50^{\prime \prime}$ just beyond the bump produced by the ring-like spiral arm. Since this break is at significantly more than two times the upper limit for the bar radius we classify the break as Type II-CT. The deep surface photometry from Pohlen et al. (2002) is consistent with the results here (cf. Fig. 3) aside from this bump produced by the outer spiral arm, which seems to be suppressed in their averaged profile. The downbending shape with a break at $\sim 50 "$ is consistent with the profile shown by van der Kruit (1987).

UGC 10721: Type III

J170825.6+253103 .S. .6? $5.8 \quad-19.68 \quad 1.23118$

Small galaxy close to our high axis ratio limit so the dust may get important. Galaxy shows an inner apparently inclined disk with a nucleus and some thin spiral arms sitting in an almost round outer isophote most probably related to a vertical structure (halo or thick disk). Thus the photometric inclination (ellipticity) and PA are highly uncertain since continously changing with radius. The shoulder at $\sim 12^{\prime \prime}$ in the final profile corresponds to an aligned spiral arm. There is a clear break visible at $\sim 40^{\prime \prime}$ followed by an upbending profile which is classified as Type III, associated to the transition between inner and outer disk (vertical structure?), so to be taken with some caution. The sharp transition in the profile argues against a thick disk. The upbending behaviour is confirmed by the profile from Courteau (1996).
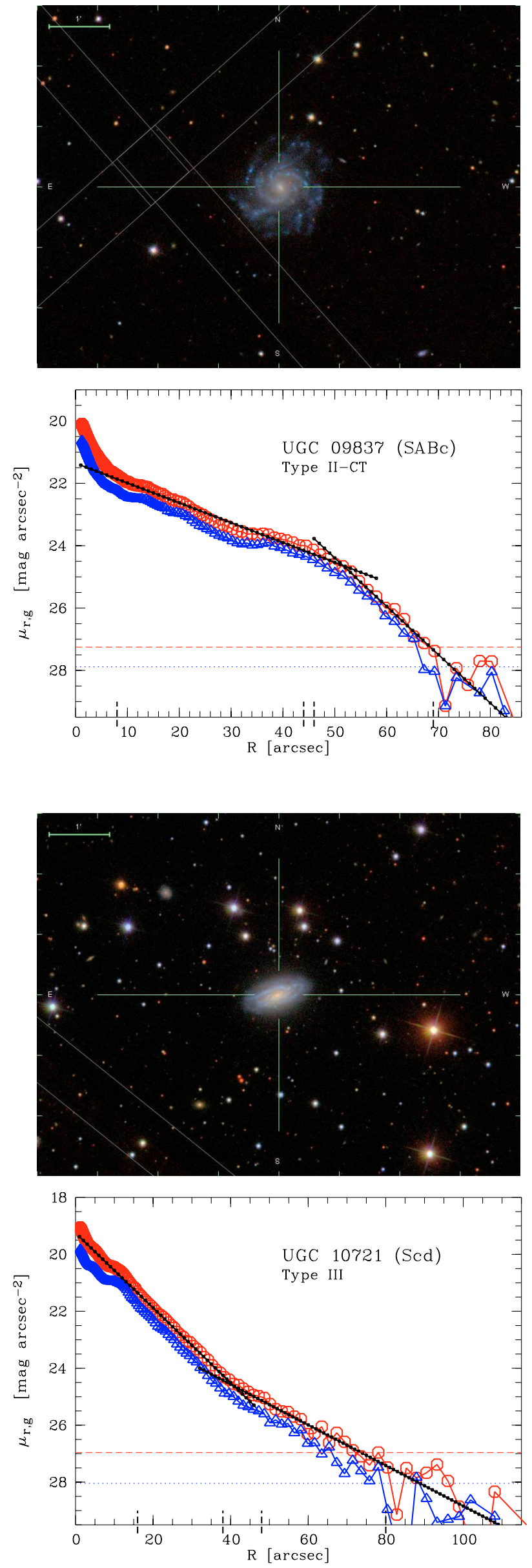
UGC 12709: Type II-CT

J233724.0+002327 .SXS9. $8.3-19.05 \quad 3.02672$

This is a faint, low surface brightness galaxy without a well defined center, so the centering (obtained from the $\sim 80^{\prime \prime}$ ellipse) and the photometric inclination (ellipticity) and PA (obtained at $\sim 70^{\prime \prime}$ ) are uncertain. The offcentered outer disk is reflected by the central dip in the final profile, which shows a clear downbending break at $\sim 70^{\prime \prime}$ corresponding roughly to the end of the flocculent starforming patches. Although classified as SAB the central region $\left(\sim 10^{\prime \prime}\right)$ is too fuzzy to argue for or against a bar, but in both cases it is to small to be responsible for the break, which is therefore classified as Type II-CT.
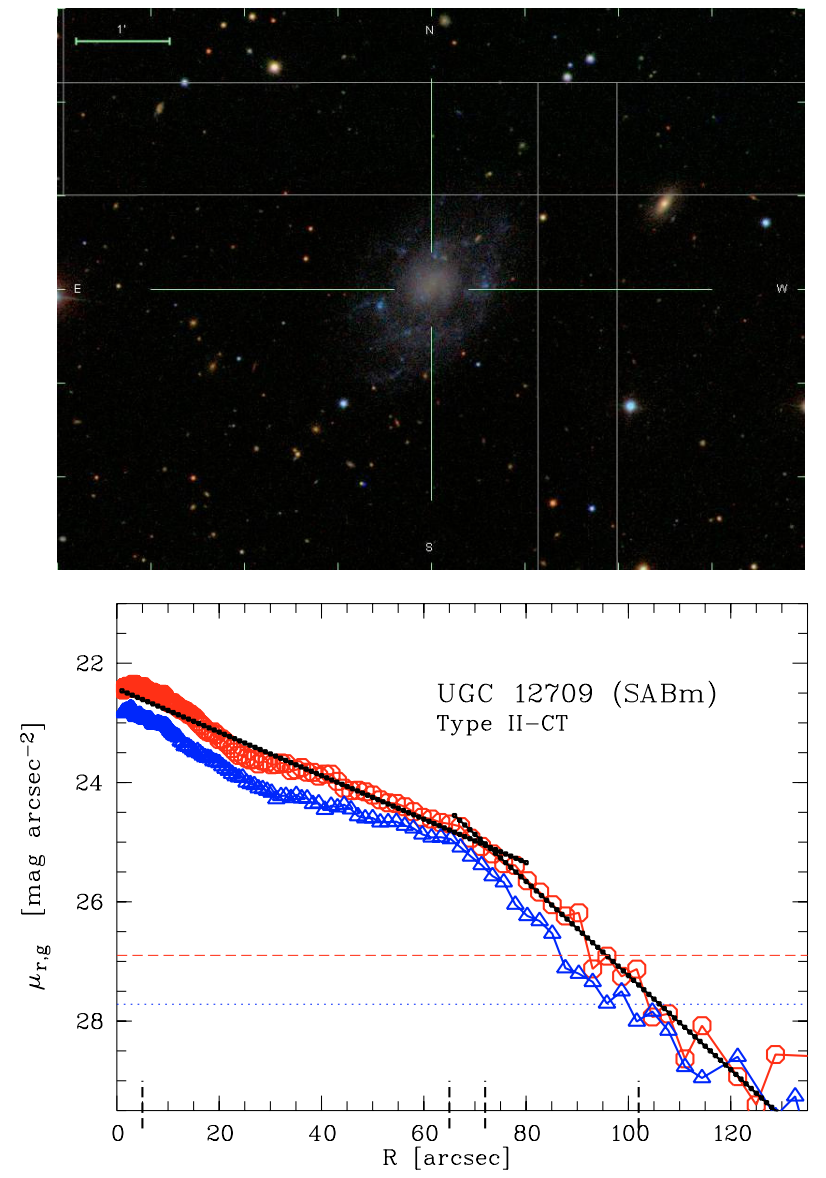


\section{Appendix B: Rejected galaxies}

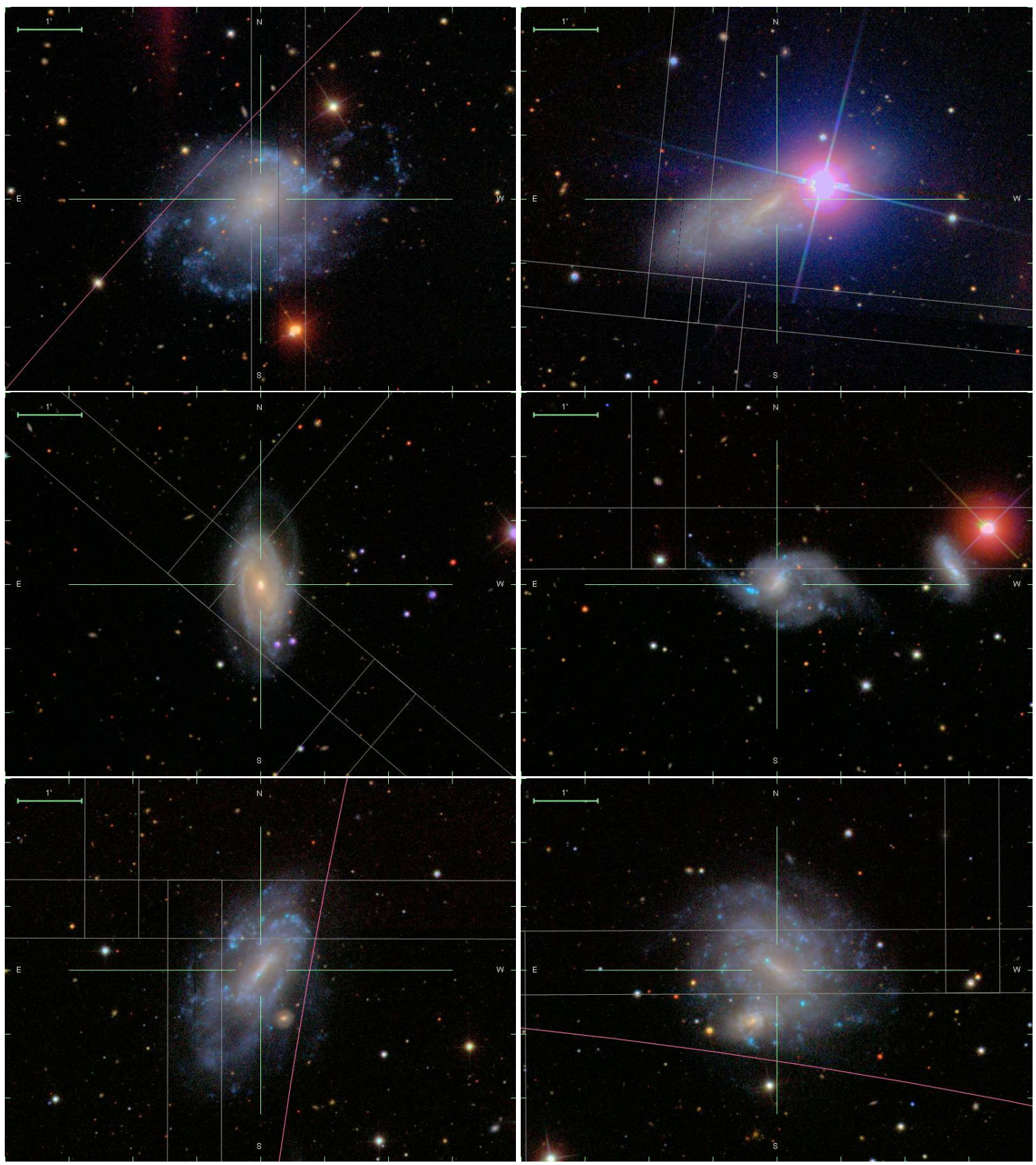

Fig. B.1. Galaxies rejected from the original LEDA-SDSS(DR2) sample: SDSS colour plates of NGC 0428, NGC 0988, NGC 2712, NGC 3023, NGC 4116, and NGC 4496A (from left to right and top to bottom). 
M. Pohlen and I. Trujillo: The structure of galactic disks, Online Material p 55

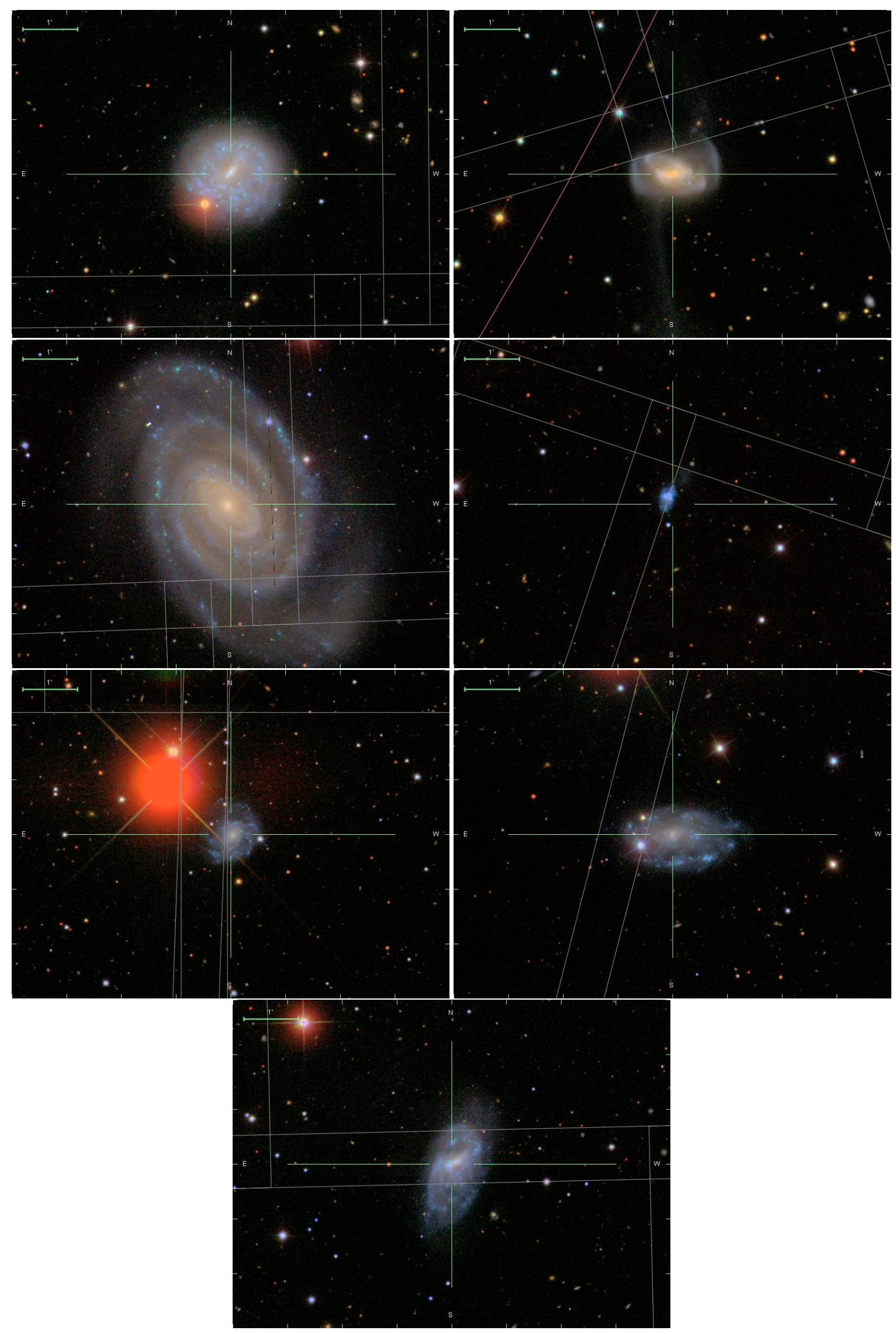

Fig. B.1. continued. Galaxies rejected from the original LEDA-SDSS(DR2) sample SDSS colour plates of NGC 4900, NGC 5218, NGC 5364, PGC 032356, UGC 04684, UGC 06162, and UGC 09215 (from left to right and top to bottom). 